\title{
TAILORING RECOMBINANT PROTEIN QUALITY BY RATIONAL MEDIA DESIGN
}

\section{DISSERTATION}

zur Erlangung des naturwissenschaftlichen Doktorgrades

der Julius-Maximilians-Universität Würzburg

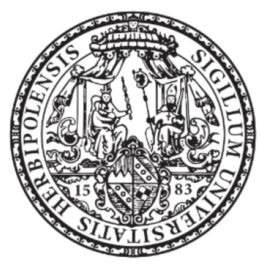

vorgelegt von DAVID BRÜHLMANN aus Baden, Schweiz 
Eingereicht am: 16.02.2017

Mitglieder der Promotionskommission:

Vorsitzender: Prof. Dr. Thomas Rudel

Gutachter: Prof. Dr. Markus Sauer

Gutachter: Prof. Dr. Jürgen Hemberger

Tag des Promotionskolloquiums: 19.04.2017

Doktorurkunde ausgehändigt am: 
To God

who so loved the world that he gave his one and only Son,

Jesus Christ, that whoever believes in him shall have eternal life.

"To raise new questions, new possibilities,

to regard old problems from a new angle,

requires creative imagination

and marks real advance in science."

— Albert Einstein 



\section{Acknowledgements}

Firstly I would like to express my sincere gratitude to my advisors Prof. Dr. Jürgen Hemberger and Prof. Dr. Markus Sauer for giving me the opportunity to carry out this $\mathrm{PhD}$ thesis focusing on recombinant protein quality modulation and for supervising the entire project. I greatly appreciate their confidence and trust in me, as well as, the granted autonomy throughout the project.

I am deeply grateful for my mentor Dr. Martin Jordan who was continuously supporting my research. Many thanks for his guidance, his immense knowledge and experience, his great out-of-the-box ideas, and his critical questions that helped me to look deeper into the research topics I was addressing.

I acknowledge Merck Biopharma in Corsier-sur-Vevey for funding this project and for giving me the opportunity to conduct the research activities. I would like to thank particularly Dr. Jonathan Souquet, Dr. Henri Kornmann, Dr. Matthieu Stettler, Jean-Marc Bielser and Dr. Hervé Broly for their great support.

Je tiens également à remercier l'équipe du laboratoire BPS-USP, particulièrement Patrick Guyot, Fabrice Schmidt, Natacha Collet, Pierre-Alain Python, Raphaël Ducommun, Emna Ben Elouja, Stéphane Ugo et Manuel Dengra pour leurs précieux conseils pratiques, leur expérience en culture cellulaire et leur aide très utile. Ce fut un immense plaisir de travailler avec eux dans un environment si chaleureux.

I acknowledge the great analytical support of the entire BPS analytical team and especially Dr. Manuel Favre and Anne-Laure Dumont. Without their support it would not have been possible to generate the plethora of results required in this project.

I am also grateful for the precious support of the cell sciences group, in particular Michel Kobr, Philippe Chatellard and Stéphane Busso as well as Luc Wertheimer and Christophe Pinel of the cell banking group.

I would like to thank the master and bachelor students Anais Muhr, Rebecca Parker, Gabrielle Leclercq, Zhigang Li, Thomas Vuillemin and Chloé Bleuez who contributed during their internship to this project.

I appreciated the knowledge sharing throughout the entire project with my colleagues Dr. 


\section{Acknowledgements}

Aline Zimmer, Dr. Nikolai Stankiewicz, Dr. Jochen Sieck and Prof. Dr. Jörg von Hagen of the Life Science Upstream R\&D group in Darmstadt.

Many thanks for the fruitful collaboration with the Department of Chemistry and Applied Biosciences, Institute for Chemical and Bioengineering of the Swiss Federal Institute of Technology at Zurich (ETHZ). I would like to thank especially Michael Sokolov, Dr. Thomas Villiger and Dr. Alessandro Butté.

A very special and warm thank you goes to my family, Karl, Ingrid, Micha, Raphael, Christina and Martina Brühlmann as well as my friends who, filled with so much love and understanding, were encouraging me throughout the entire $\mathrm{PhD}$ thesis. 


\section{Abstract}

Nowadays, more than half of the biotherapeutics are produced in mammalian cell lines as a result of correct protein folding and assembly as well as their faculty to bring about a variety of post-translational modifications. The widespread progression of biosimilars has moved the focus in mammalian cell-culture process development. Thereby, the modulation of quality attributes of recombinant therapeutic proteins has increasingly gained importance from early process development stages. Protein quality directly shapes the clinical efficacy and safety in vivo, and therefore, the control of the complex post-translational modifications, such as glycosylation (e.g. high mannose, fucosylation, galactosylation and sialylation), charge variants, aggregates and low-molecular-weight species formation, is pivotal for efficient receptor binding and for triggering the desired immune responses in patients. In the frame of biosimilar development, product quality modulation methods using the potential of the host cell line are particularly sought after to match the quality profile of the targeted reference medicinal product (RMP) as closely as possible. The environment the cell is dwelling in directly influences its metabolism and the resulting quality profile of the expressed protein. Thereby the cell culture medium plays a central role in upstream manufacturing. In this work, concentration adjustment of selected media components and supplementation with a variety of compounds was performed to alter various metabolic pathways, enzyme activities and in some cases the gene expression levels of Chinese Hamster Ovary (CHO) cells in culture. The supplementation of cell culture medium with the trisaccharide raffinose in fed-batch cultures entailed an increase of the abundance of high mannose glycans in two different $\mathrm{CHO}$ cell lines. Raffinose especially favored mannose 5 glycans. At the same time, it impaired cell culture performance, induced changes on the intracellular nucleotide levels and even varied the expression levels of glycosylation-related genes. Supplementation with a number of galactosyltransferase inhibiting compounds, in particular fluorinated galactose analogs ( $\alpha$ - and $\beta$-2F-peracetyl-galactose), consistently decreased the production of galactosylated monoclonal antibodies (mAb). By means of targeted addition during the culture rather than at the beginning, the inhibition was further increased, while limiting detrimental effects on both growth and productivity. High-throughput screening in 96-deepwell plates showed that spermine and L-ornithine also reduced the level of galactosylation. On the other hand, exploratory screening of a variety of potentially disulfide-bridge-reducing agents highlighted that the inherent low-molecular-species level of the proprietary platform cell culture process was likely due to favored reduction. This hypothesis was reinforced by the observation that supplementation of cysteine and $\mathrm{N}$-acetylcysteine promoted fragmentation. Additionally, 
fragmentation decreased with higher protein expression.

At that point, aiming to improve the efficiency in process development, a rational experimental design method was developed to identify and to define the optimal concentration range of quality modulating compounds by calling on a combination of high throughput fed-batch testing and multivariate data analysis. Seventeen medium supplements were tested in five parallel 96-deepwell plate experiments. The selection process of promising modulators for the follow-up experiment in shake tubes consisted in a three-step procedure, including principal component analysis, quantitative evaluation of their performance with respect to the specifications for biosimilarity and selection following a hierarchical order of decisions using a decision tree. The method resulted in a substantial improvement of the targeted glycosylation profile in only two experimental rounds. Subsequent development stages, namely validation and transfer to industrial-scale facilities require tight control of product quality. Accordingly, further mechanistic understanding of the underlying processes was acquired by non-targeted metabolomic profiling of a $\mathrm{CHO}$ cell line expressing a mAb cultured in four distinct process formats. Univariate analysis of intra- and extracellular metabolite and temporal glycosylation profiles provided insights in various pathways. The numerous of parameters were the main driver to carry out principal component analysis, and then, using the methodology of partial-least-square (PLS) projection on latent structures, a multivariate model was built to correlate the extracellular data with the distinct glycosylation profiles. The PLS observation model proved to be reliable and showed its great benefit for glycan pattern control in routine manufacturing, especially at large scale. Rather than relying on post-production interpretation of glycosylation results, glycosylation can be predicted in real-time based on the extracellular metabolite levels in the bioreactor.

Finally, for the bioactivity assessment of the glycan differences between the biosimilar and the reference medicinal product (RMP), the health agencies may ask for in the drug registration process, extended ranges of glycan variants need to be generated so that the in vitro assays pick up the changes. The developed glycosylation modulator library enabled the generation of extreme glycosylation variants, including high mannose, afucosylated, galactosylated as well as sialic acid species of both a mAb and an antibody fusion molecule with three $\mathrm{N}$ glycosylation sites. Moreover, to create increased variety, enzymatic glycoengineering was explored for galactosylation and sialylation. The glyco variants induced significant responses in the respective in vitro biological activity assays. The data of this work highlight the immense potential of cell culture medium optimization to adjust product quality. Medium and feed supplementation of a variety of compounds resulted in reproducible and important changes of the product quality profile of both mAbs and a fusion antibody. In addition to the intermediate modulation ranges that largely met the requirements for new-biological-entity and biosimilar development, medium supplementation even enabled quick and straightforward generation of extreme glycan variants suitable for biological activity testing.

Keywords: CHO cell culture, product quality modulation, media design, metabolism, glycosylation, high throughput 


\section{Zusammenfassung}

Mehr als die Hälfte der Biotherapeutika werden heutzutage aufgrund korrekter Proteinfaltung und korrektem Zusammenbau in tierischen Zelllinien hergestellt, welche zudem die Fähigkeit besitzen, verschiedene posttranslationale Modifikationen zu bewerkstelligen, hergestellt. Der ausgeprägte Aufschwung von Biosimilars hat den Entwicklungsschwerpunkt von Zellkulturverfahren verlagert. Dabei hat die Modulierung der Qualitätsattribute von rekombinanten Proteinen bereits in frühen Entwicklungsstadien eine wichtige Bedeutung erlangt. Die Qualitätsattribute beeinflussen die klinische Wirksamkeit und die In-Vivo-Sicherheit direkt. Somit ist die Regulierung der posttranslationalen Modifikationen, einschließlich der Glykosylierung (mannosereiche, fukosylierte, galaktosylierte und sialylierte Glykane), der Ladungsvarianten, sowie die Bildung von Aggregaten und niedermolekularen Spezien, für effiziente Rezeptorbindung und das Auslösen der gewünschten Immunantwort in Patienten entscheidend. Im Rahmen der Biosimilarentwicklung werden Methoden zur Anpassung der Produktqualität innerhalb des Potentials der Wirtszelle gesucht, um sie möglichst genau dem Referenzarzneimittel anzugleichen. Die Umgebung, in der die Zelle verweilt, beeinflusst ihren Metabolismus und das resultierende Produktqualitätsprofil. Dabei spielen Medien eine zentrale Rolle in der Zellkultur. Im Rahmen dieser Doktorarbeit wurden durch Adjustierung von ausgewählten Medienbestandteilen und Ergänzung mit einer Vielfalt von Stoffen diverse Stoffwechselwege, Enzymaktivitäten und in einigen Fällen das Genexpressionsniveau von kultivierten Chinesischen Hamster-Ovarialzellen (CHO) verändert. Die Ergänzung von Zellkulturmedium mit Raffinose, ein Trisaccharid, führte zu einer Erhöhung des mannosereichen Glykosylierungsmusters in zwei unterschiedlichen CHO-Zelllinien. Raffinose begünstigte hauptsächlich Mannose-5-Spezien. Gleichzeitig wurde die Zellkulturleistung beeinträchtigt und zudem intrazelluläre Nukleotidkonzentrationen sowie das Expressionsniveau von Glykosylierungsgenen verändert. Ergänzung mit mehreren Inhibitoren der Galaktosyltransferase, insbesondere fluorierte Galaktosenachbildungen ( $\alpha$ - und $\beta$-2F-Peracetyl-Galaktose), verringerte stetig die Produktion von galaktosylierten monoklonalen Antikörpern (mAb). Durch gezielte Zugabe im Verlauf der Kultur, statt bereits am Anfang, wurde die Inhibition weiter erhöht, und dabei die Einwirkung auf das Zellwachstum und die Produktivität beschränkt. Ein Hochdurchsatz-Screening in 96-Deep-Well-Platten zeigte, dass Spermin und L-Ornithin auch das Ausmaß der Galaktosylierung reduzierte. Andererseits zeigten erste Nachforschungen anhand eines Screenings einer Auswahl von potenziellen Disulfidbrücken-Reduktionsmittel, dass wahrscheinlich begünstigte Reduktion das inhärente Niedermolekular-Speziesniveau des firmeneigenen Zellkulturplattformverfahrens verursacht. Die Hypothese wurde durch die 
Beigabe von Cystein und N-Acetylcystein bekräftigt. Diese Stoffe begünstigten die Fragmentierung, wohingegen sie bei höherer Proteinexpression abnahm.

Mit dem Ziel die Entwicklungseffizienz zu steigern, wurde daraufhin zur Identifikation von qualitätsverändernden Stoffen und Bestimmung der optimalen Konzentrationsbereichen eine rationale Versuchsanordnungsmethode entwickelt. Dazu wurde eine Kombination von Hochdurchsatz-Fed-Batch-Tests und multivariater Datenanalyse herbeigezogen. Siebzehn Mediumergänzungsstoffe wurden in fünf parallelen 96-Deep-Well-Platten-Experimenten getestet. Das Auswahlverfahren von erfolgsversprechenden Modulatoren fürs Nachfolgeexperiment in Schüttelröhrchen umfasste drei Schritte: Hauptkomponentenanalyse, quantitative Evaluierung der Leistung der Modulatoren hinsichtlich der Biosimilaritätsspezifikationen und die Auswahl in Anlehnung an eine hierarchische Entscheidungsreihenfolge mit Hilfe eines Entscheidungsbaums. Die Methode führte in nur zwei Versuchsreihen zu einer erheblichen Annäherung an das gewünschte Glykosylierungsprofil. Anschließende Entwicklungsschritte (Validierung und Transfer in die großtechnische Anlage) erforden eine rigorose Kontrolle der Produktqualität. Demzufolge konnte dank der Non-Targeted Metabolomics Analyse von vier verschiedenen Herstellungsverfahren einer mAb exprimierenden CHO-Zelllinie weitere mechanistische Kenntnisse der zugrunde liegenden Vorgängen gewonnen werden. Univariate Analysen der intra- und extrazellulären Stoffwechselprodukte und die zeitliche Glykosylierungsprofile lieferten einen Einblick in verschiedene Stoffwechselwege. Die Vielzahl von Parametern führte dazu, nach dem Prinzip der Hauptkomponentenanalyse vorzugehen, und dann anhand der Partial Least Squares (PLS)-Projektion auf latente Strukturen ein multivariates Modell zu erstellen, das die extrazellulären Daten mit den individuellen Glykosylierungsprofilen korreliert. Das PLS Beobachtungsmodell stellte sich als verlässlich heraus und zeigte seinen außerordentlichen Nutzen zur Regulierung der Glykanen in der Routineherstellung, insbesondere in der Großanlage. Anstatt sich auf Glykosylierungsresultate nach dem Ende der Produktion zu verlassen, kann die Glykosylierung, basierend auf den Niveaus der extrazellulären Stoffwechselprodukte im Bioreaktor, in Echtzeit vorausgesagt werden.

Schließlich können im Rahmen des Arzneigenehmigungsverfahrens Gesundheitsbehörden verlangen, die Glykanunterschiede zwischen dem Biosimilar und dem Referenzarzneimittel zu untersuchen. Damit der biologische Test die Unterschiede nachweisen kann, muss eine erweiterte Palette von Glykanvarianten hergestellt werden. Die entwickelte Glykosylierungsmodulierungsbibliothek ermöglichte, extreme Varianten für mannosereiche, afukosylierte, galaktosylierte und sialylierte Glykane von mAb und einem Antikörperfusionsmolekül mit drei N-Glykosylierungsstellen zu generieren. Für erhöhte Variantenvielfalt wurde die enzymatische Glykoengineering Technologie für die Galaktosylierung und Sialylierung untersucht. Die Glykanvarianten erzeugten signifikante Antworten in der jeweiligen In-Vitro-Bestimmung der biologischen Aktivität. Die Ergebnisse unterstreichen das immense Potential von Zellkulturmediumoptimierung zur Anpassung der Produktqualität. Ergänzung des Mediums und der Nährstofflösung brachte reproduzierbare und beträchtliche Veränderungen der Produktqualität von mAb und eines Fusionsantikörpers hervor. Zusätzlich zu den intermediären Modulierungsbereichen, die mehr als ausreichend den Anforderungen für die Entwicklung von neuen biologischen Wirkstoffen und Biosimilars genügen, ermöglichte die Mediumergänzung 
auf schnelle und einfache Art und Weise selbst extreme Glykanvarianten zu bilden, die für die Bestimmung der biologischen Aktivität geeignet waren.

Stichwörter: CHO Zellkultur, Produktqualitätsmodulierung, Mediendesign, Metabolismus, Glykosylierung, hoher Durchsatz 



\section{Résumé}

De nos jours, plus de la moitié des biothérapeutiques sont produites dans des lignées cellulaires de mammifère en raison de leur efficacité à mener à bien le repliement et l'assemblage des protéines ainsi que de leur faculté d'entraîner une variété de modifications posttraductionnelles. L'essor croissant des biosimilaires a changé les priorités dans le développement de procédés de culture cellulaire mammifère. Ainsi, la modulation des attributs de qualité de protéines recombinantes thérapeutiques est devenue de plus en plus importante. Et cela depuis le début du développement car la qualité de la protéine influence l'efficacité clinique et la sûreté in vivo. Par conséquent, le contrôle des modifications post-traductionnelles telles que la glycosylation (glycans riches en mannose, fucose, galactose et acide sialique), les variants de charges et la formation d'agrégats et d'espèces de bas poids moléculaire est essentiel pour la liaison efficace aux récepteurs et pour le déclenchement de la réponse immunitaire désirée chez les patients. Dans le cadre du développement de biosimilaires, des méthodes de modulation de la qualité du produit en utilisant le potentiel de la cellule hôte sont particulièrement recherchées pour obtenir un profil de qualité le plus semblable possible au produit médical de référence. L'environnement dans lequel la cellule réside influence de manière directe son métabolisme et la qualité du produit qui en résulte. Ainsi, le milieu joue un rôle central dans la culture cellulaire. Dans le cadre de cette thèse, grâce à l'ajustement de certains composés du milieu ainsi qu'à la supplémentation en une variété de composés, différentes voies métaboliques, activités enzymatiques et dans certains cas les niveaux d'expression de certains gènes chez les cellules d'ovaire de hamster chinois (CHO) en culture ont été modifiés. La supplémentation du milieu de culture en raffinose, un trisaccharide, a entraîné une augmentation des niveaux de glycans riches en mannose dans deux lignées CHO différentes. Favorisant surtout les glycans de type mannose 5, le raffinose avait aussi des effets défavorables sur la performance de la culture cellulaire. De plus, il a induit des changements de niveaux des nucléotides intracellulaires et a même varié les niveaux d'expression de gènes relatifs à la glycosylation. La supplémentation en divers inhibiteurs de la galactosyltransferase, à savoir des analogues fluorinés du galactose ( $\alpha$ - et $\beta$-2F-peracétyl-galactose) a invariablement diminué la production d'anticorps monoclonaux (mAb) galactosylés. Grâce à la supplémentation ciblée au cours de la culture plutôt qu'au début, l'inhibition est devenue plus prononcée tandis que les effets négatifs sur la croissance et la productivité ont été limités. Un criblage à haut débit en plaque 96 puits a montré que la spermine et la L-ornithine ont également diminué le taux de galactosylation. D'autre part, un criblage exploratoire d'une variété d'agents réducteurs potentiels de ponts disulfures a démontré que le niveau de forme de bas poids moléculaire 
inhérent au procédé de culture cellulaire plateforme propriétaire serait le résultat d'une réduction favorisée. Cette hypothèse a été renforcée par l'observation que la fragmentation s'est accentuée en cas de supplémentation en cystéine et en $\mathrm{N}$-acétylcystéine. Par ailleurs, la fragmentation a diminué lorsque l'expression de protéine a augmenté.

A ce stade, afin d'améliorer l'efficacité du développement de procédés, une méthode de conception d'expériences rationnelle a été développée, ayant comme objectif d'identifier et de définir la gamme de concentration optimale de composés modulant la qualité. Afin d'y parvenir, une combinaison d'expérimentation à haut débit en fed batch et d'analyse multivariée a été utilisée. Dix-sept suppléments de milieu ont été testés en cinq expériences parallèles en plaque 96 puits. Le processus de sélection des modulateurs prometteurs pour l'expérience en tubes agités (shake tubes) s'est articulé en trois étapes, notamment l'analyse des composantes principales, l'évaluation quantitative de leur performance relative aux spécifications de biosimilarité et enfin leur sélection selon un ordre hiérarchique en utilisant un arbre de décision. La méthodologie a conduit à une amélioration considérable du ciblage du profil de glycosylation en uniquement deux séries d'expériences. Les étapes de développement ultérieures, à savoir la validation et le transfert à l'échelle industrielle, exigent un contrôle rigoureux des attributs de qualité. Ainsi, le profilage métabolomique non ciblé dans quatre formats distincts de procédé à partir d'une lignée CHO exprimant un mAb a permis d'obtenir une compréhension supplémentaire des mécanismes sous-jacents. Des analyses univariées des profils de métabolites intra- et extracellulaires ainsi que des profils de glycosylations au cours du temps ont fourni une connaissance approfondie de plusieurs voies métaboliques. Les innombrables paramètres ont conduit à l'analyse des composantes principales et ensuite à la création d'un modèle afin de corréler les données extracellulaires aux profils de glycosylation grâce à la méthodologie de la projection des moindres carrés (PLS) aux structures latentes. Le modèle d'observation PLS s'est avéré fiable et a montré son avantage pour le contrôle du profil de glycosylation en routine, et particulièrement à grande échelle. Au lieu de se fier à l'interprétation à postériori des résultats de glycosylation, le profil peut être prédit en temps réel à partir des niveaux de métabolites extracellulaires du bioréacteur.

Enfin, les autorités de santé peuvent demander une évaluation de l'effet sur la bioactivité dû aux différences dans le profil de glycosylation entre le biosimilaire et le médicament de référence. Des gammes de variants de glycans doivent être produites pour que les méthodes analytiques détectent la différence. La bibliothèque de modulateurs de glycosylation développée a permis de générer des variants de glycosylation extrêmes, y compris des formes riches en mannose, des formes afucosylés, galactosylés et sialylées d'un anticorps et d'une molécule d'anticorps de fusion munie de trois sites de N-glycosylation. Afin de créer une plus grande variété, le traitement enzymatique a été évalué pour la galactosylation et la sialylation. Les variants de glycosylation ont induit des réponses significatives dans les analyses de bioactivité in vitro. Nos données mettent l'accent sur l'immense potentiel de l'optimisation du milieu de culture cellulaire afin d'ajuster la qualité du produit. La supplémentation du milieu et de la solution d'alimentation en plusieurs composés a généré des changements reproductibles et importants du profil de qualité d'un anticorps et d'un anticorps de fusion. Outre les gammes de modulation intermédiaires largement suffisantes dans le cadre du développement de nou- 
velles entités biologiques et de biosimilares, la supplémentation de milieu a même rendu possible la génération rapide et simple de variants de glycosylation extrême qui conviennent à l'analyse de l'activité biologique.

Mots clefs : culture cellulaire $\mathrm{CHO}$, modulation de la qualité de produit, développement de milieu, métabolisme, glycosylation, haut débit 



\section{Contents}

$\begin{array}{ll}\text { Acknowledgements } & \text { i }\end{array}$

Abstract (English/Deutsch/Français)

List of figures $\quad$ xvii

List of tables $\quad$ Xxv

Introduction 1

I State of the Art 3

1 Cell Culture Process Optimization 5

2 The Potential of Media to Enhance Protein Quality 9

2.1 Glycosylation . . . . . . . . . . . . . . . . . . . . . . 10

2.1.1 Non Specific Impact of Media Components _ . . . . . . . . . . . . . 12

2.1.2 High Mannose Species . . . . . . . . . . . . . . . . . . . . . . . . 13

2.1 .3 Fucosylation . . . . . . . . . . . . . . . . . . . . . . 16

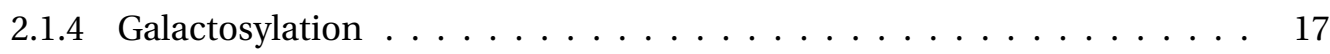

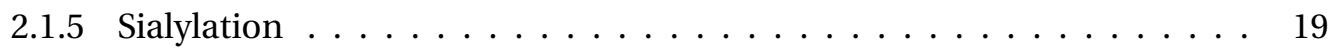

2.1 .6 Glycation . . . . . . . . . . . . . . . . . . . . . 20

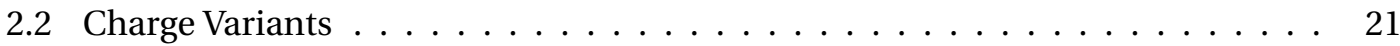

2.2 .1 Deamidation \& Isomerization $\ldots \ldots \ldots \ldots \ldots$

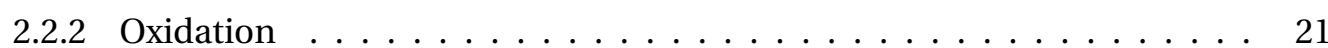

2.2 .3 C- and N-Terminal Modifications _ . . . . . . . . . . . . . . 22

2.2.4 Arginine Modifications by Methylglyoxal . . . . . . . . . . . . . . 23

2.2.5 Global Acidic Species Charge Variant Modulation . . . . . . . . . . . . . 23

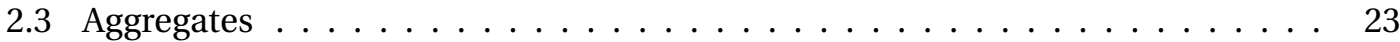

2.4 Low-Molecular-Weight Species . . . . . . . . . . . . . . . . . . . . 25

2.5 Amino Acid Misincorporation . . . . . . . . . . . . . . . . . . . 26

2.6 Components Affecting Multiple Quality Attributes . . . . . . . . . . . . . . 27

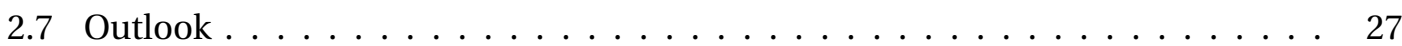


$\begin{array}{lr}\text { II Research } & 29\end{array}$

3 Research Objectives $\quad 31$

4 High Mannose Increase $\quad 33$

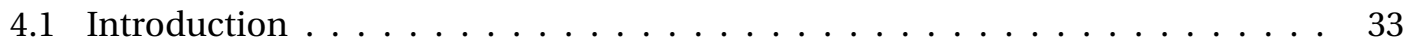

4.2 Materials and Methods . . . . . . . . . . . . . . . . . . . 34

4.2 .1 Inoculum Preparation . . . . . . . . . . . . . . . . 34

4.2.2 Cell Culture in 96-Deepwell Plates . . . . . . . . . . . . . . . 35

4.2 .3 Cell Culture in Shake Tubes . . . . . . . . . . . . . . . . . . 35

4.2.4 Cell Culture in 3.5-L Bioreactors . . . . . . . . . . . . . . 36

4.2.5 Cell Counts, Cell Viability and mAb Titer Analysis . . . . . . . . . . . . 36

4.2 .6 Glycan Analysis . . . . . . . . . . . . . . . . . . . . . 36

4.2.7 Intracellular Nucleotide and Nucleotide Sugar Profiling . . . . . . . . 36

4.2 .8 Transcriptomics Analysis . . . . . . . . . . . . . . . . . 37

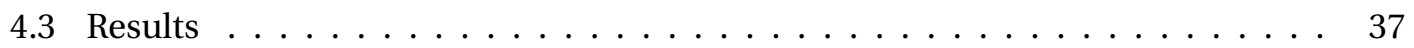

4.3.1 Cultures in 96-Deepwell Plates . . . . . . . . . . . . . . 37

4.3.2 Cultures at Constant Medium Osmolality . . . . . . . . . . . . . . . . 40

4.3.3 Cultures in Shake Tubes and 3.5-L Bioreactors . . . . . . . . . . . . . . 40

4.3.4 Analysis of Nucleotides, Nucleotide Sugars and Transcriptomics . . . . . 42

4.4 Discussion . . . . . . . . . . . . . . . . . . . 42

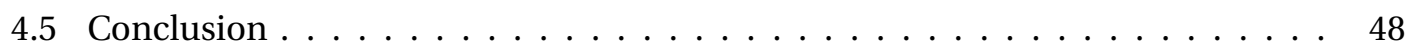

5 Specific Inhibition of Galactosylation $\quad 49$

5.1 Introduction . . . . . . . . . . . . . . . . . . . . 49

5.2 Materials and Methods . . . . . . . . . . . . . . . 51

5.2 .1 Inoculum Preparation . . . . . . . . . . . . . . . 51

5.2 .2 Cell Culture Conditions . . . . . . . . . . . . . . . . 51

5.2.3 Analytical Methods for Cell Culture Performance . . . . . . . . . . . . 52

5.2 .4 Glycan Analysis . . . . . . . . . . . . . . . . . . . . 53

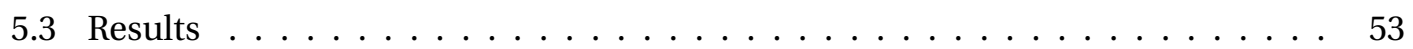

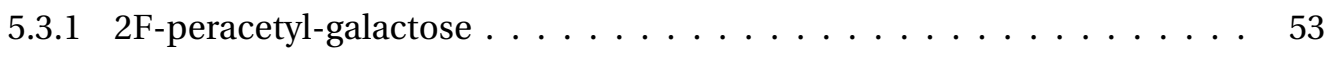

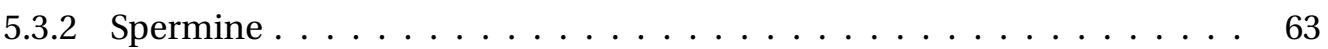

5.3 .3 L-ornithine . . . . . . . . . . . . . . . . . 65

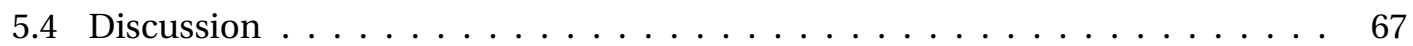

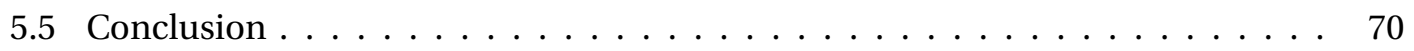

6 Low-Molecular-Weight Species $\quad 71$

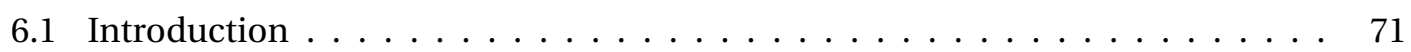

6.2 Materials and Methods . . . . . . . . . . . . . . . . . 73

6.2 .1 Inoculum Preparation . . . . . . . . . . . . . 73

6.2 .2 Cell Culture Conditions . . . . . . . . . . . . . . . 73

6.2.3 Analytical Methods for Cell Culture Performance . . . . . . . . . . . . 75 
6.2.4 Analysis of Low-Molecular-Weight Species Content . . . . . . . . . . . . 75

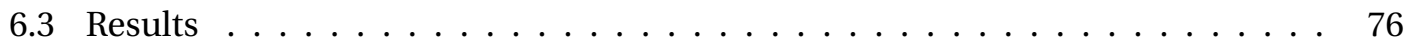

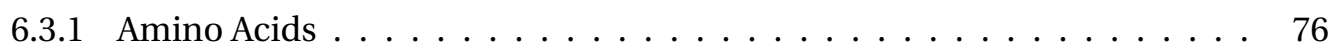

$6.3 .2 \quad \mathrm{~N}$-Acetyl-Cysteine $\ldots \ldots \ldots \ldots \ldots \ldots$

6.3 .3 Chelating Agents $\ldots \ldots \ldots \ldots$. . . . . . . . . . 83

6.3 .4 Metal Ions $\ldots \ldots \ldots \ldots \ldots \ldots \ldots \ldots \ldots \ldots \ldots \ldots \ldots$

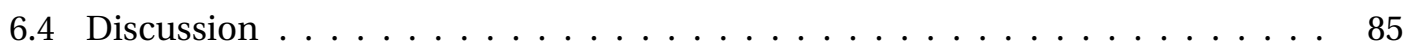

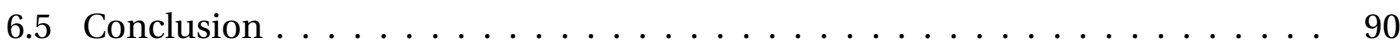

7 Parallel Experimental Design and Multivariate Analysis $\quad 91$

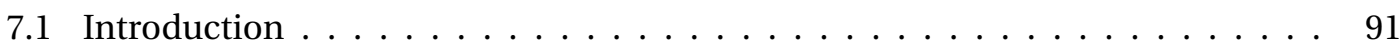

7.2 Materials and Methods . . . . . . . . . . . . . . . . . . . . 93

7.2 .1 Inoculum Preparation $\ldots \ldots \ldots \ldots \ldots \ldots \ldots$

7.2.2 Cell Culture in 96-deepwell Plates and Experimental Design . . . . . . . 93

7.2.3 Cell Culture in Shake Tubes and Experimental Design . . . . . . . . . . 94

7.2.4 Product Quality Analysis . . . . . . . . . . . . . . . . . . . . . . . 95

7.2 .5 Data Analysis . . . . . . . . . . . . . . . . . . . 95

7.3 Results and Discussion . . . . . . . . . . . . . . . . . . . . 96

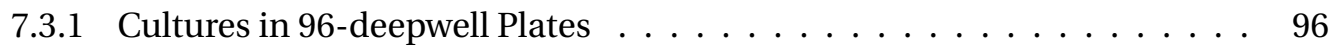

7.3.2 Identification of the Best Glycosylation Modulators . . . . . . . . . . . . 97

7.3.3 Verification and Extension in Shake Tubes . . . . . . . . . . . . . . . 102

7.4 Conclusions . . . . . . . . . . . . . . . . . . . . . . . . . . . . . . . 105

7.5 Acknowledgement . . . . . . . . . . . . . . . . . . . . 107

8 Linking Metabolomic Profiling with Glycosylation 109

8.1 Introduction . . . . . . . . . . . . . . . . . . . . . . . . . . . . . 109

8.2 Materials and Methods . . . . . . . . . . . . . . . . . . . . . . . . 111

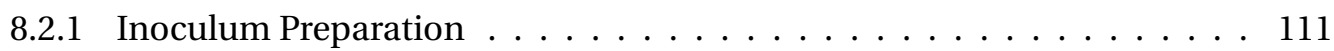

8.2.2 Cell Culture in 3.5-L Bioreactors ～. . . . . . . . . . . . . . . . 111

8.2.3 Analytical Methods for Cell Culture Performance . . . . . . . . . . . 112

8.2.4 Glycan Analysis . . . . . . . . . . . . . . . . . . . . . . . . . . . 112

8.2.5 Non-Targeted Metabolite Profiling . . . . . . . . . . . . . . . . 112

8.2.6 Multivariate Analysis . . . . . . . . . . . . . . . . . . . . . . . . 113

8.3 Results . . . . . . . . . . . . . . . . . . . . . . . . 115

8.3.1 Non-targeted Profiling of Intra- and Extracellular Metabolites . . . . . 115

8.3.2 Temporal Glycosylation and Nucleotide Sugar Profiles . . . . . . . . . . 121

8.3.3 Multivariate Analysis and Modelling . . . . . . . . . . . . . . . . . . . 124

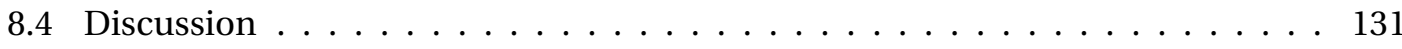

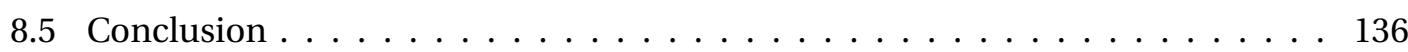


9 Glycan Variants for Bioactivity Testing $\quad 137$

9.1 Introduction . . . . . . . . . . . . . . . . . . . . . . . . 137

9.2 Materials and Methods . . . . . . . . . . . . . . . . . . . . . . 138

9.2 .1 Inoculum Preparation . . . . . . . . . . . . . . . . 138

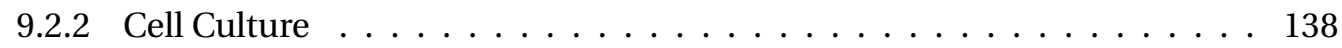

9.2 .3 Enzymatic Glycoengineering . . . . . . . . . . . . . . . . . . . 139

9.2.4 Analytical Methods for Cell Culture Performance . . . . . . . . . . . . . 139

9.2 .5 Glycan Analysis . . . . . . . . . . . . . . . . . . . . . . . . . 140

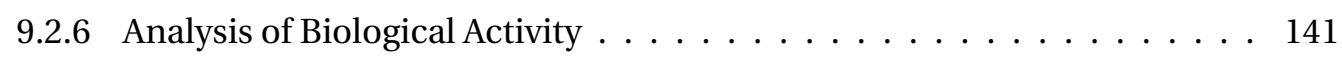

9.3 Results . . . . . . . . . . . . . . . . . . . . . . . . . 144

9.3.1 Cell Culture Performance . . . . . . . . . . . . . . . . . . . . 144

9.3 .2 Glycan Distribution . . . . . . . . . . . . . . . . . . . . . 145

9.3 .3 Biological Activity . . . . . . . . . . . . . . . . . . . . . . 149

9.4 Discussion . . . . . . . . . . . . . . . . . . . . . . . . . 154

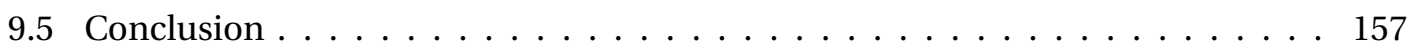

$\begin{array}{lr}10 \text { Concluding Remarks and Perspectives } & 159\end{array}$

$\begin{array}{ll}\text { A Experimental Designs } & 163\end{array}$

$\begin{array}{ll}\text { Bibliography } & 197\end{array}$

$\begin{array}{lr}\text { Nomenclature } & 199\end{array}$

$\begin{array}{lr}\text { Scientific Contributions } & 205\end{array}$

$\begin{array}{lr}\text { Declaration of Authorship } & 209\end{array}$ 


\section{List of Figures}

1.1 Parameters affecting process performance and recombinant protein quality attributes. A non-exhaustive list of examples for each parameter category is

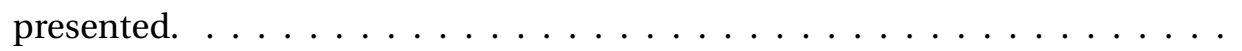

2.1 Schematic N-glycosylation pathway in the endoplasmic reticulum (ER) and Golgi apparatus. . . . . . . . . . . . . . . . . . . . . . . . . 11

4.1 Fold glycosylation change in function of raffinose concentration $(0-50 \mathrm{mM})$ in the production medium prior to 96-DWP inoculation with cell line 1 (A) and cell line 2 (B). The control cultures $(0 \mathrm{mM})$ were conducted in 4 replicates and raffinose supplemented conditions in duplicates. Error bars show variability within replicates. HM: high mannoses, Fuc: fucosylated species, Gal: galactosylated species, Misc: miscellaneous. . . . . . . . . . . . . . . . . . . . . . .

4.2 (A) Viable cell densities of cell line 1 cultures supplemented with 0-50 $\mathrm{mM}$ of raffinose. (B) Viable cell densities of cell line 2 cultures supplemented with 0$50 \mathrm{mM}$ of raffinose. (C) Harvest titers (day 14) of cell line 1 cultures. (D) Harvest titers (day 14) of cell line 2 cultures. All raffinose supplemented cultures were performed in duplicates and the control in four replicates in 96-DWP. . . . . . .

4.3 (A) Viable cell densities of cell line 2 cultures supplemented with $0-128 \mathrm{mM}$ of raffinose. (B) Harvest titers (day 14) of cell line 2 cultures. All raffinose supplemented cultures were performed in replicates as indicated in legend of figure A in 96-DWP. The medium osmolality after raffinose addition was adjusted to $315 \mathrm{mOsm} / \mathrm{kg}$, which corresponds to the osmolality of the non-supplemented

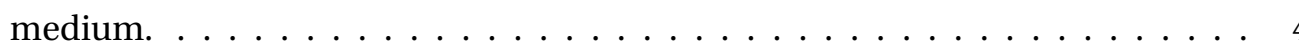


4.4 (A) High mannose glycan levels of shake tubes at harvest. All conditions ( 0,10 , 30, 50 and $100 \mathrm{mM}$ raffinose) were carried out in duplicates, using cell line 1 at constant medium osmolality ( $315 \mathrm{mOsm} / \mathrm{kg}$ ). Error bars represent glycan variability within one condition. (B) High mannose glycan levels of shake tubes at harvest of Cellvento cultures. All conditions (0, 10, 30 and $50 \mathrm{mM}$ raffinose) were carried out in duplicates using cell line 1. Error bars represent glycan variability within one condition. (C) High mannose glycan levels of shake tubes at harvest (cell line 2). All conditions $(0,10,50$ and $100 \mathrm{mM}$ raffinose) were carried out at constant medium osmolality $(315 \mathrm{mOsm} / \mathrm{kg})$ in duplicates. Error bars represent glycan variability within one condition. . . . . . . . . . .

4.5 High mannose levels in 3.5-L bioreactor runs with cell line 1 at $0 \mathrm{mM}$ (control), 15 and $30 \mathrm{mM}$ raffinose in the medium $(\mathrm{n}=1) \ldots \ldots \ldots \ldots$

4.6 High mannose level at three medium osmolalities (300, 315 and $375 \mathrm{mOsm} / \mathrm{kg}$ ) in the absence of raffinose and with $30 \mathrm{mM}$ raffinose supplementation $(+\mathrm{R})$ in cell line 2 cultures performed in $96-$ DWP . . . . . . . . . . . . .

4.7 Level of intracellular nucleotides and nucleotide sugars (cell line 1) in ST runs at $0 \mathrm{mM}$ (control), 15, 30, 50 and $100 \mathrm{mM}$ raffinose in the medium on culture day 3 . The error bars indicate the standard deviation of the technical duplicates. . . .

5.1 (A) Viable cell densities of cell line A cultures supplemented with $0-200 \mu \mathrm{M}$ of $\alpha$ 2F-peracetyl-galactose. (B) Viabilities. (C) Product titers on day 10. (D) Product titers in the harvest on day 14. The number of replicates of each condition is indicated in chart A. All points are mean values of the corresponding replicates and the error bars report the standard deviation of the replicates. . . . . . . .

5.2 (A) Absolute change of the overall glycosylation pattern compared to the control in function of the $\alpha$-2F-p-galactose concentration in medium. (B) Absolute change of galactosylation compared to the control in function of the $\alpha-2 \mathrm{~F}-\mathrm{p}$ galactose concentration in medium. All points are mean values of the corresponding replicates analyzed by CGE-LIF and the error bars report the standard

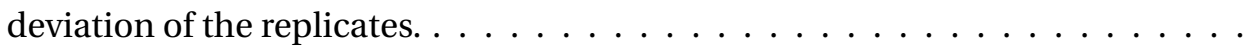

5.3 (A) Viable cell densities of cell line A cultures supplemented with $0-200 \mu \mathrm{M}$ of $\beta$-2F-peracetyl-galactose. (B) Harvest titer (day 14). The number of replicates of each condition is indicated in chart A. All points are mean values of the corresponding replicates and the error bars report the standard deviation of the

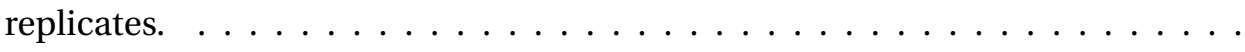

5.4 (A) Absolute change of the overall glycosylation pattern compared to the control in function of the $\beta$-2F-p-galactose concentration in medium. (B) Absolute change of galactosylation compared to the control in function of the $\beta-2 \mathrm{~F}-\mathrm{p}$ galactose concentration in medium. All points are mean values of the corresponding replicates analyzed by CGE-LIF and the error bars report the standard deviation of the replicates. . . . . . . . . . . . . . . . . . 58

xviii 
5.5 (A) Viable cell densities of cell line A cultures supplemented with 0-90 $\mu \mathrm{M} \alpha-2 \mathrm{~F}$ peracetyl-galactose, $60 \mu \mathrm{M} \beta$-2F-peracetyl-galactose, or $10 \mathrm{mM}$ ammonium in ST. (B) Viabilities. (C) Protein titer for each concentration on culture days 5, 7, 10, 12 and 14. Each condition was conducted in duplicates. All points are mean values of the corresponding conditions and the error bars report the maximum

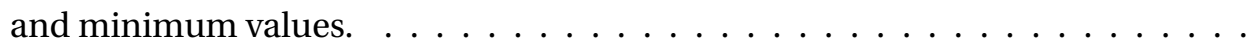

5.6 (A) Absolute change of the overall glycosylation pattern compared to the control in function of the $\alpha$ - and $\beta$-2F-p-galactose concentration in medium in comparison with $10 \mathrm{mM}$ ammonium in cell line A cultures. (B) Absolute change of galactosylation compared to the control in function of the $\alpha$ - and $\beta-2 \mathrm{~F}-\mathrm{p}$ galactose concentration in medium in comparison with $10 \mathrm{mM}$ ammonium. Each condition was conducted in duplicates. All bars represent mean values of the corresponding conditions analyzed by CGE-LIF and the error bars report the maximum and minimum values. . . . . . . . . . . . . . . . . .

5.7 (A) Viable cell densities of cell line B cultures supplemented with 0-90 $\mu \mathrm{M} \alpha-2 \mathrm{~F}$ peracetyl-galactose in ST. (B) Viabilities. (C) Protein titer for each concentration on culture days 5, 7, 10, 12 and 14. Each condition was conducted in duplicates. All points are mean values of the corresponding conditions and the error bars report the maximum and minimum values. . . . . . . . . . . . . .

5.8 (A) Absolute change of the overall glycosylation pattern compared to the control in function of the $\alpha-2 \mathrm{~F}$-p-galactose concentration in medium of cell line B cultures. (B) Absolute change of galactosylation compared to the control in function of the $\alpha$-2F-p-galactose concentration in medium of cell line B cultures. Each condition was conducted in duplicates and analyzed by 2AB-UPLC. All bars represent mean values of the corresponding conditions and the error bars report the maximum and minimum values. . . . . . . . . . . . .

5.9 (A) Viable cell densities of cell line A cultures in function of the feed timing of $\beta$-2F-p-galactose in ST. (B) Viabilities. (C) Protein titer for each condition on culture days 5, 7, 10, 12 and 14. Experiments were conducted in duplicates. All points are mean values of the corresponding conditions and the error bars report the maximum and minimum values. . . . . . . . . . . . . . . . . . . .

5.10 (A) Absolute change of the overall glycosylation pattern compared to the control in function of the feed timing of $\beta-2 \mathrm{~F}$-p-galactose in cell line A cultures. (B) Absolute change of galactosylation compared to the control in function of the feed timing of $\beta$-2F-p-galactose in cell line A cultures. Experiments were conducted in duplicates and supernatant analyzed by CGE-LIF. All bars represent mean values of the corresponding conditions and the error bars report the maximum

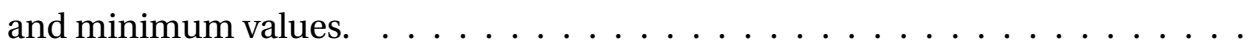


5.11 (A) Viable cell densities of cell line A cultures supplemented with $0-200 \mu \mathrm{M}$ of spermine. (B) Viabilities. (C) Product titers on day 10. (D) The titer in the harvest on day 14. The number of replicates of each condition is indicated in chart A. All points are mean values of the corresponding replicates and the error bars report the standard deviation of the replicates. . . . . . . . . . . . . .

5.12 (A) Absolute change of the overall glycosylation pattern compared to the control in function of the spermine concentration in the medium. (B) Absolute change of galactosylation compared to the control in function of the spermine concentration in the medium. All points are mean values of the corresponding replicates analyzed by CGE-LIF and the error bars report the standard deviation

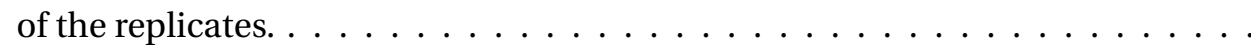

5.13 (A) Viable cell densities of cell line A cultures supplemented with 0-15 mM of L-ornithine. (B) Viabilities. (C) Product titers on day 10. (D) The titer in the harvest on day 14. The number of replicates of each condition is indicated in chart A. All points are mean values of the corresponding replicates and the error bars report the standard deviation of the replicates. . . . . . . . . . . . .

5.14 (A) Absolute change of the overall glycosylation pattern compared to the control in function of the L-ornithine concentration in the medium. (B) Absolute change of galactosylation compared to the control in function of the L-ornithine concentration in the medium. All points are mean values of the corresponding replicates analyzed by CGE-LIF and the error bars report the standard deviation

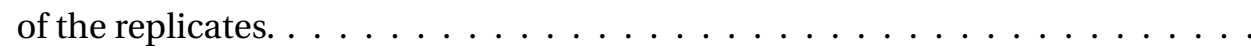

6.1 (A) LMW levels of cell line A cultures in 96-DWP in function of the cysteine concentration increase in the medium prior to inoculation, including the linear regression line (equation: $\operatorname{LMW}(\%)=1.722+0.1517$ cysteine $\left.(\mathrm{mM}), R^{2}=61.9 \%\right)$. (B) LMW levels of cell line A cultures in 96-DWP in function of the cysteine concentration in the CD-feed added on days $3,5,7,10$, and 12 . The linear regression line is shown as well (equation: $\operatorname{LMW}(\%)=4.405+0.09167$ cysteine

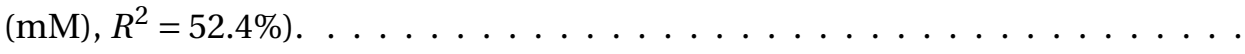

6.2 (A) LMW levels of cell line B cultures in 96-DWP in function of the cysteine concentration increase in the medium prior to inoculation, including the linear regression line (equation: $\operatorname{LMW}(\%)=2.593+0.1377$ cysteine $(\mathrm{mM}), R^{2}=36.4 \%$ ). (B) LMW levels of cell line B cultures in 96-DWP in function of the cysteine concentration in the CD-feed added on days $3,5,7,10$, and 12 . The linear regression line is shown as well (equation: $\operatorname{LMW}(\%)=4.434+0.03361$ cysteine

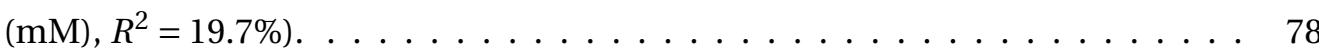


6.3 (A) LMW content cell line A cultures in 96-DWP in function the cell line A protein titer at the end of the culture (day 14), including the linear regression line (equation: $\operatorname{LMW}(\%)=2.425-0.000424$ titer $\left.(\mathrm{mg} / \mathrm{L}), R^{2}=40.7 \%\right)$. The cysteine supplemented cultures are highlighted (gray circle). (B) LMW content cell line B cultures in 96-DWP in function the cell line A protein titer at the end of the culture (day 14), including the linear regression line (equation: $\operatorname{LMW}(\%)=3.145-0.000276$ titer $\left.(\mathrm{mg} / \mathrm{L}), R^{2}=25.9 \%\right)$. The cysteine supplemented cultures are highlighted (gray circle) $\ldots \ldots \ldots \ldots$

6.4 LMW content in function of the cysteine concentration increase in the medium prior to inoculation. Each condition was performed in duplicates. All bars represent mean values of the corresponding conditions and the error bars report

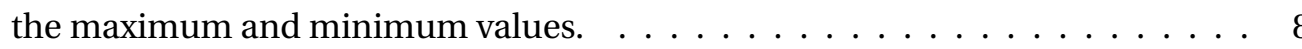

6.5 Electropherogram of one control sample and one of the culture supplemented with $50 \mathrm{mM}$ cysteine. . . . . . . . . . . . . . . . . . . . . . .

6.6 LMW content in function of the $\mathrm{N}$-acetyl-cysteine concentration in the medium prior to inoculation. Each condition was performed in duplicates. All bars represent mean values of the corresponding conditions and the error bars report the maximum and minimum values. $\ldots \ldots \ldots \ldots \ldots$

6.7 Electropherogram of one control sample and one of the culture supplemented with $2 \mathrm{mM} \mathrm{N}$-acetyl-cysteine. . . . . . . . . . . . . . . . .

6.8 LMW content in function of the EDTA and DMSA concentrations in the medium prior to inoculation. Each condition was performed in duplicates. All bars represent mean values of the corresponding conditions and the error bars report the maximum and minimum values. The control culture was analyzed in a separate sequence than the remainder of the supplemented conditions. . . . .

6.9 Electropherogram of one control sample and one of the culture supplemented with 2.2 mM DMSA. . . . . . . . . . . . . . . . . . . . . . 84

6.10 LMW content in function of the ferric ammonium citrate concentration increase in the medium prior to inoculation. Each condition was performed in duplicates. All bars represent mean values of the corresponding conditions and the error bars report the maximum and minimum values. . . . . . . . . . . . 85

6.11 Electropherogram of one control sample and one of the culture supplemented

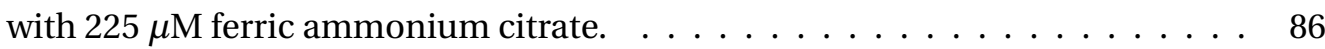

6.12 LMW content in function of the $\mathrm{CuSO}_{4}$ and $\mathrm{ZnSO}_{4}$ concentration increases in the medium prior to inoculation. Each condition was performed in duplicates. All bars represent mean values of the corresponding conditions and the error bars report the maximum and minimum values. $\ldots \ldots \ldots \ldots$

7.1 Sequential design of experiments method using characteristic compound groups and multivariate selection of best quality modulating compounds. . . . . . . . 96 
7.2 Boxplots of glycan modulation ranges. The group independent control samples were conducted in 8 replicates: 4 on each 96-DWP plate. The dashed lines mark the respective specification ranges, where applicable. (A) High mannose glycan modulation ranges in each group (1-5). Man4 to Man7 were detected and summed up. (B) Modulation ranges of afucosylated species including A0, A1 and $\mathrm{A} 2$ in the five groups. (C-E) The three charts present agalactosylated species (FA2), the sum of monogalactosylated species FA2[3]G1 and FA2[6]G1 as well as the abundance of digalactosylated glycan (FA2G2). (F) The sialylated forms FA2G2S1, FA2G2S2 and FA2G2S1(NGNA) were grouped in one single chart. . .

7.3 Boxplots showing the range of charge variants and aggregation levels. The group independent control samples were conducted in 8 replicates: 4 on each 96-DWP plate. (A-E) The charge variants were grouped into 5 clusters: acidic (1-2), neutral (3), basic (4-5). The charts show the corresponding ranges within the five groups.

(F) The aggregate ranges of each group are displayed. . . . . . . . . . .

7.4 (A) Score plot for joint PCA of 96-DWP experiments (light gray) and of ST experiments (deep gray) with projected optimum. The first two PCs are shown explaining almost $50 \%$ of the total variance. Ellipses show equidistant conditions according to Mahalanobis distance ( 1 to 4 distance units in the plain of the first two PCs). (B) Boxplots showing distance to optimum for 96-DWP and ST experiments based on first 3 PCs. PC3 explains additional 16\% of variance. The plus symbols mark outliers. . . . . . . . . . . . . . . . . . . . . . 100

7.5 Boxplots showing Mahalanobis distance to optimum in function of the concentration level of four compounds in 96-DWP experiments. The plus symbols mark

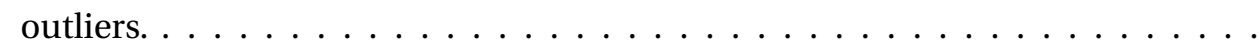

7.6 Pruned decision tree for selection of best glycosylation modulators. At each node the number of observations (regular), and the average distance to the target (bold) is provided. The concentration level of the decision variable is shown in italic. . . . . . . . . . . . . . . . . .

7.7 (A) Boxplots of glycan modulation ranges obtained with raffinose, galactose and enhancer 2 in ST bioreactor tubes at $36.5^{\circ} \mathrm{C}(\mathrm{H})$ and when lowering the temperature to $33^{\circ} \mathrm{C}$ on culture day $5(\mathrm{~L})$. The dashed lines represent the specification ranges, where applicable. (B) Boxplots of the charge variants: acidic (clusters 1 \& 2, neutral (cluster 3), basic (clusters 4 \& 5). (C) Boxplots of aggregates and low molecular species (LMW) . . . . . . . . . . . . . . . . . . 103

7.8 Boxplots showing Mahalanobis distance to optimum in function of the concentration levels of the compounds and the culture temperature from day 5 of ST experiments. Plus symbols mark outliers. . . . . . . . . . . . .

8.1 (A) Viable cell densities. (B) Viabilities. (C) Product titer. (D) Extracellular glucose concentration prior to feeding. (E) Extracellular lactate concentration. (F) Extracellular ammonium concentration. All four runs were conducted in 3.5-L bioreactors for 14 days. . . . . . . . . . . . . . . . . . . 117

xxii 
8.2 Intracellular (above) and extracellular (below) asparagine profiles. . . . . . . . 118

8.3 Intracellular (above) and extracellular (below) homocysteine profiles. . . . . . . 119

8.4 Intracellular (above) and extracellular (below) alanine profiles. . . . . . . . . . 120

8.5 Levels of FA2, FA2G1 (sum of FA2[3]G1 and FA2[6]G1) and FA2G2 in processes A to $\mathrm{D}$ as a relation of the culture time. The glycosylation profile was analysed daily from culture 3 to 14 by 2AB-UPLC. . . . . . . . . . . . . . . . . . . . . . . 121

8.6 (A) Ratio between di-galactosylated (FA2G2) and mono-galactosylated (FA2G1) forms in function of the culture day. (B) Sum of agalactosylated (FA2), monogalactosylated (FA2G1) and di-galactosylated forms (FA2G2) in function of the culture day. . . . . . . . . . . . . . . . . . . . . . . . . . 122

8.7 (A) Intracellular UDP-GlcNAc profile throughout the cell culture of processes A to D. (B) Intracellular UDP-glucose profile throughout the cell culture of processes A to D. . . . . . . . . . . . . . . . . . . . . . . . 123

8.8 PCA-X score plot of processes A to D. The PC1 explains $44 \%$ of variance, and PC2 7\%. Each time point is labelled with the respective culture hour of the corresponding process format. The ellipse delimits the $0.95 \%$-confidence area.

8.9 PLS scatter plot of processes A, B and D. The goodness of fit $\left(R^{2} X\right)$ of the first component amounts to $39 \%$ and the second component to $10 \%$. Each time point is labelled with the respective culture hour of the corresponding process format. The ellipse delimits the $0.95 \%$-confidence area. . . . . . . . . . . . . . . . 127

8.10 PLS weight plot of processes A, B and D. The goodness of fit $\left(R^{2} X\right)$ of the first component amounts to $39 \%$ and the second component to $10 \%$. . . . . . . . 128

8.11 Variable importance plot. The chart includes the variables above 1 . The error bars indicate the $95 \%$ confidence intervals. . . . . . . . . . . . . . . . . . . 129

8.12 Observed versus predicted values of FA2, FA2[6]G1, FA2[3]G1 and FA2G2 . . . . 130

8.13 Experimental and predicted glycans of process $\mathrm{C}$ by PLS model on days 6 (A) and 14 (B). The model was built with extracellular metabolite data of process A, $\mathrm{B}$ and $\mathrm{D}$. The dashed box marks the calibration set range of the corresponding glycan. . . . . . . . . . . . . . . . . . . . . . . . . 132

8.14 Comparison of experimental FA2 values versus predicted values of the four different models indicated in the chart legend. The first three letters indicate the processes included in the calibration set of the model, while the last corresponds to the predicted process. . . . . . . . . . . . . . . . . . . .

9.1 (A) Viable cell densities of cell line A cultures supplemented with glycosylation modulators and non-supplemented control culture. The control and the kifunensine supplemented cultures were conducted in triplicates $(n=3)$, while the others in duplicates $(n=2)$. (B) Viabilities. (C) Product titers on days 10, 12 and 14. In all charts average values of the replicates are shown. The error bars indicate the upper and lower limits of the values. . . . . . . . . . . . . . . . 146 
9.2 (A) Viable cell densities of cell line B cultures supplemented with glycosylation modulators and non-supplemented control culture. The experiment was conducted in two independent series. At the exception of $30 \mu \mathrm{M}$ kifunensine, the conditions belong to series 1 . Both control 1 and the control of the second series (control 2) were conducted in triplicates $(n=3)$. All supplemented cultures of series 1 and 2 were performed in duplicates $(n=2)$. (B) Viabilities. $(C)$ Product titers on days 5, 7, 10 and 12. All charts show average values of the replicates. The error bars indicate the upper and lower limits of the replicate values. . . . . 147

9.3 Glycan pattern of control and cultures supplemented with either raffinose, kifunensine, 2F-p-fucose, manganese \& galactose, or ammonium (cell line A). . . . 148

9.4 Glycan pattern of control and cultures supplemented with either raffinose, kifunensine, 2F-p-fucose, manganese \& galactose, or ammonium (cell line B). Each chart corresponds to one of the three glycan sites. (A) Glycan site 1 located at Fc domain. (B) Glycan site 2 located at non-Fc-part of fusion entity. (C) Glycan site 3 located at non-Fc-part of fusion entity. . . . . . . . . . . . . 150

9.5 Level of total afucosylated and fucosylated species at the three glycosylation sites of the antibody fusion molecule expressed by cell line B. Both afucosylated and fucosylated forms include galactosylated and sialylated forms. High mannose species were not considered. . . . . . . . . . . . . . . . . . 151

9.6 Glycan antennarity of control and culture supplemented with 2F-p-fucose of cell line B. (A) Glycan site 1 located at Fc domain. (B) Glycan site 2 located at non-Fc-part of fusion entity. (C) Glycan site 3 located at non-Fc-part of fusion entity. The number of branches is shown on the right-hand side of the charts. . 152

9.7 (A) Fc $\gamma$ RIIIa F158 affinity of cell line A. (B) Fc $\gamma$ RIIIa V158 affinity. (C) Fc $\gamma$ RIIIb affinity. (D) Relative ADCC reporter potency. (E) Relative Clq potency. (F) Relative CDC potency. The results were released as averages of independent assays. The error bar mark the variability. . . . . . . . . . . . . . . . 153

9.8 (A) Fc $\gamma$ RIIIa F158 affinity. (B) Fc $\gamma$ RIIIa V158 affinity. (C) Fusion entity cellbased activity. (D) Fusion entity activity by Biacore. The results were released as averages of three independent assays. The error bar mark the variability. . . . . 154

A.1 PCA score plot of 96-DWP experiments. The PC1 explains $35 \%$ of variance, and PC2 24\%. The different experiments are marked with their DoE group. . . . . 163

A.2 PCA loading plot of 96-DWP experiments. . . . . . . . . . . . . . . . . . 164

A.3 PCA on 96-DWP experiments: Cumulative variance explained by the PCs (solid line) and Scree plot showing variance explained by each PC (dashed line). The characteristic elbow at $\mathrm{PC}=4$ indicates that the relevant information is likely to be captured by the first 3 PCs. . . . . . . . . . . . . . . . . . . . . . . . 164 


\section{List of Tables}

2.1 Specific and/or potent inhibitors, activators of glycosylation processing steps as well as compounds increasing the availability of the precursor. . . . . . . . . 12

2.2 Media components affecting multiple quality attributes simultaneously. . . . . 28

4.1 Expression of genes involved in the glycosylation pathway in ST supplemented with $100 \mathrm{mM}$ raffinose. All values are relative to the non-supplemented condition and expressed in $\log _{2}$-fold changes. . . . . . . . . . . . .

5.1 Concentrations of glycosylation modulating compounds in the cell culture

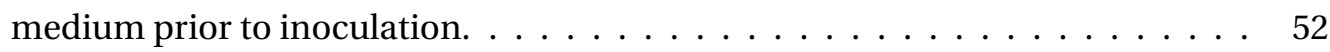

5.2 Feeding regime of $\mathrm{ST}$ feed optimization experiments. . . . . . . . . . . . . 52

5.3 Glycan grouping calculation for CGE-LIF data. . . . . . . . . . . . . . . 53

5.4 Glycan grouping calculation for $2 \mathrm{AB}-U \mathrm{ULC}$ data $\ldots \ldots \ldots \ldots$

6.1 Concentration ranges of cell culture medium supplements in medium prior to

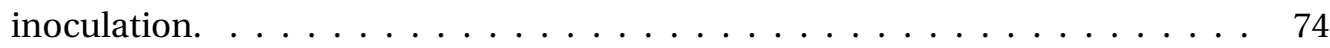

6.2 Concentrations of chelating agents added to the supernatant before harvesting

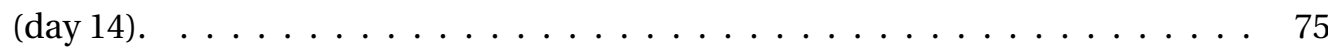

7.1 Group factor concentrations in medium prior to inoculation of 96-DWP. . . . . 94

7.2 Comparison of fulfillment of the specifications for biosimilarity of experiments in 96-DWP and ST. The structure of each glycan is shown: $\mathrm{N}$-acetylglucosamine (blue square), mannose (green circle), fucose (red triangle), galactose (purple circle). For each cell culture system the percentage of experiments reaching the optimum for the corresponding glycan are presented. . . . . . . . . . . . 106

8.1 Media supplementation of the four 3.5-L bioreactor fed-batch processes. . . . . 111

8.2 Feed supplementation of the four 3.5-L bioreactor fed-batch processes. . . . . . 112

8.3 Goodness of fit $\left(R^{2} X\right.$ and $\left.R^{2} Y\right)$ and goodness of prediction $\left(Q_{\text {cum }}^{2}\right)$ of PLS model in function of the number of latent variables. . . . . . . . . . . . . 126

8.4 Average PLS observation model errors of each glycan species \pm 2 standard deviations taking into account all four models. . . . . . . . . . . . . . . . 131

9.1 Medium supplement concentrations prior to inoculation. . . . . . . . . . . . 139 


\section{List of Tables}

9.2 Glycan sites of antibody (cell line A) and fusion antibody (cell line B). . . . . . 140

9.3 Glycan grouping for cell line A. . . . . . . . . . . . . . . . . . . . . . 141

9.4 Glycan grouping for cell line B. . . . . . . . . . . . . . . . . . . . . . . . 142

A.1 Experimental design of group 1 in 96 DWP. . . . . . . . . . . . . . . . 165

A.2 Experimental design of group 2 in 96 DWP. . . . . . . . . . . . . . . . . . . 166

A.3 Experimental design of group 3 in 96 DWP. . . . . . . . . . . . . . . . . . . 167

A.4 Experimental design of group 4 in 96 DWP. . . . . . . . . . . . . . . . . 168

A.5 Experimental design of group 5 in 96 DWP. . . . . . . . . . . . . . . . . . . . 169

A.6 Design of experiments in TubeSpin bioreactor tubes. . . . . . . . . . . . . . 170 


\section{Introduction}

In the early 1980s the pharmaceutical industry embarked on a remarkable journey when the first recombinant drug, recombinant insulin produced in E. coli, was approved by the Food and Drug Administration (FDA) ${ }^{1,2}$. Patients have benefited ever since from safer and more efficacious drug products due to recombinant analogs rather than proteins of animal origin, which enabled substantial purity improvements ${ }^{3}$. The advent of biotherapeutics revolutionized the landscape of the healthcare sector, and as a consequence of the great improvements they brought about, the biologics market has massively grown ${ }^{4}$, resulting in tripling of new biological license applications in the beginning of the $21^{\text {st }}$ century and reached all-time records in 2012, 2014 and 2015, exceeding twelve per year ${ }^{5}$. Nowadays, more than $50 \%$ of the approved recombinant therapeutics are produced in mammalian cell lines, including Chinese hamster ovary cells, baby hamster kidney cells, and mouse myeloma cells, such as NS0 and SP2/0, as well as human cell lines ${ }^{6}$. Among them, $\mathrm{CHO}$ cells are the most frequently employed in the biotech industry, which represents more than $60 \%$ of the approved biologics that are produced in mammalian cell lines ${ }^{6,7}$. As a result of the high level of characterization of $\mathrm{CHO}$ and its successful use in the production of a variety of products over many years, it will likely continue, in the near future, to be the expression system of choice ${ }^{7}$. While the overall cell culture principles in industry have remained unchanged since the mid-1980s, improvements of media compositions, including complex feeds entailed dramatic yield increases, in particular in fed-batch processes ${ }^{8,9}$. Ensuing the patent expiry of best-selling biological molecules many companies have pursued biosimilar development, and thus, tools to modulate the quality attributes of recombinant proteins have gained much interest from early process development stages on ${ }^{10}$. The structural characteristics of recombinant therapeutic proteins including a variety of complex post-translational modifications have a direct link with their safety and inherent biological activity in vivo ${ }^{11-13}$. Within the array of these modifications, glycosylation most strongly affects pharmacokinetics and protein physiochemical properties ${ }^{14}$. More specifically in the case of a monoclonal antibody, the presence of the carbohydrate structure interposed between the $\mathrm{CH} 2$ domain is pivotal for effector system interactions ${ }^{15}$. A variety of parameters-the cell line, the culture conditions and the cell culture medium compositionshape recombinant protein quality attributes ${ }^{16-18}$. The environment that surrounds the cell while in suspension, namely the cell culture medium, plays a key role in the cell metabolism, and for this reason, media design lends itself to alter the key quality attributes of the molecule 
within the potential of a given cell line ${ }^{19-22}$. The adjustment of the levels of a choice of medium components and the addition of novel compounds either in the medium or the in bolus feeds, influence the enzymatic reactions in the endoplasmic reticulum and in the Golgi apparatus, the substrate generation as well as their transport into the two organelles ${ }^{23,24}$. Further enhancements and technology advancements of high-throughput cell culture systems, including 96-deepwell plates and TubeSpin bioreactor tubes, allow to test a plethora of different supplements at various concentrations simultaneously ${ }^{25-27}$. Moreover, the observed trends in these systems may be reproduced in controlled bioreactor systems, including lab-scale bioreactors ${ }^{28}$, which highlights the great value of small-scale high-throughput compound screening for the identification of new levers that tailor recombinant protein quality attributes. 


\section{Part I}

\section{State of the Art ${ }^{1}$}

1. This part was published in a slightly different version: D. Brühlmann, M. Jordan, J. Hemberger, M. Sauer, M. Stettler, H. Broly, Tailoring recombinant protein quality by rational media design, Biotechnology Progress 31 (3) (2015) 615-629. It was also updated with the latest publications (2015-2016). 



\section{Chapter 1}

\section{Cell Culture Process Optimization}

The biopharmaceutical industry has substantially progressed since the approval of recombinant insulin, the first recombinant drug, in the early 1980s by the Food and Drug Administration (FDA) ${ }^{1,2}$. More than $50 \%$ of the remarkable number of approved recombinant therapeutics on the market today are being manufactured in mammalian cell lines ${ }^{1,6}$. They encompass several rodent-derived cell lines Chinese hamster ovary (CHO) cells, baby hamster kidney (BHK) cells, and mouse myeloma cells, including NS0 and SP2/0, as well as human cell lines (HEK293, HT-1080) ${ }^{6}$. Chinese hamster ovary (CHO) cells are the most used mammalian cell line ${ }^{7}$, accounting for the production of more than $60 \%$ of mammalian cell culture derived currently approved biologics ${ }^{6}$. Very well characterized, $\mathrm{CHO}$ are likely to stay the expression system of choice as they have been successfully used to produce a variety of clinical biopharmaceuticals ${ }^{7}$. A record of 12 novel biologics license applications (BLAs) were approved only in $2014^{5}$. In a CHO Consortium article entitled Recombinant Protein Therapeutics from CHO Cells-20 Years and Counting ${ }^{29} \mathrm{~K}$. Jayapal stated: "Recombinant protein therapeutics have changed the face of modern medicine in the past decade, and they continue to provide innovative and effective therapies for numerous previously refractory illnesses."

While the basic concepts of cell culture have remained unchanged since the mid-1980s ${ }^{8}$, improvements in the media compositions, including complex feeds opened the way for a dramatic yield increase ${ }^{9}$. Back then, batch production processes of about 7 days reached a cell density of $2 \times 10^{6}$ cells $/ \mathrm{mL}$ with a specific productivity slightly below $10 \mathrm{pg} / \mathrm{cell} /$ day and a final titer of $50 \mathrm{mg} / \mathrm{L}^{8}$. For many years the biopharmaceutical industry was aiming to increase specific productivity to minimize production cost-of-goods while maintaining product quality $^{30}$. Several parameters including the biology of the production cell line, product quality and stability, manufacturing capacity, process scalability, volumetric productivity, and unit cost determine the selection of the production process, namely fed-batch or perfusion ${ }^{31}$. Due to product stability issues, many recombinant proteins, such as insulin and interferons, were produced in perfusion in the early stages of the biotechnology industry ${ }^{32}$. Product residence times are low in perfusion mode and thus the recombinant drug is less exposed to various 
side-products such as proteases affecting the product integrity and high temperature. When antibodies began to enter the development pipelines, the biotech manufacturing changed over to fed-batch mainly due to productivity reasons. Antibody therapies may require large doses over a long period of time ${ }^{33}$. In that context, many companies have built large-scale facilities of working volumes $\geq 10,000 \mathrm{~L}$. In the mid-2000, product titers of $5 \mathrm{~g} / \mathrm{L}$ at the end of the production have become the industry standard ${ }^{8}$. Nowadays, biotechnology companies are reporting productivities as high as $10-13 \mathrm{~g} / \mathrm{L}$ in a fed-batch culture of $2-3$ weeks ${ }^{33,34}$.

Despite the substantially increased productivity of fed-batch processes and manufacturing capacity, the latter has become an issue because of the constantly increasing demand of specific and thus efficacious biotherapeutics ${ }^{9,31}$. Further capacity increases by either building new facilities or by process intensification (eg. high seeding processes) are certainly a way to secure the supply of innovative treatments ${ }^{35}$. On the other hand, perfusion has gained much interest, once again ${ }^{36}$. The productivity of newly developed perfusion processes is much greater than what those developed in the 80's achieved, and more importantly, volume exchanges are low (1-2 reactor volumes per day), which potentially drives cost of goods down $^{35,37,38}$. Furthermore, the continuous mode can be extended to the purification platform, thus reducing the total processing time and the risks associated with degradation pathways ${ }^{32}$.

Recently, the focus of cell-culture process development began to shift from productivity and cell growth towards the modulation of quality attributes of recombinant therapeutic proteins from early process development stages on, in particular in the frame of biosimilar development, which many companies are pursuing due to patent expiry of biologics ${ }^{10}$. The efficacy, potency and safety depend on the structural characteristics of the protein entity ${ }^{11}$, which explains the particular attention paid in biotherapeutic development to post-translational modifications that the expressed protein may undergo ${ }^{1,12,14,39-41}$. In the particular case of monoclonal antibodies (mAbs), increased antibody-dependent cell-mediated cytotoxicity (ADCC) is one of the major objectives to increase the immune response of the human body and thus clinical efficacy ${ }^{42}$. Many studies have addressed various effects of glycosylation and charge variants on the biological activity and pharmacokinetics ${ }^{11,40,43,44}$. On that account, it is of utmost importance to understand the glycosylation process and how it is influenced by cell culture variations in bioprocess development and manufacturing in order to manage to control and optimize the glycan pattern ${ }^{24}$. As a result, efforts to develop techniques to alter the properties of therapeutic proteins have multiplied, aiming to improve clinical utility with respect to antigen targeting and potency ${ }^{40}$.

Both selection and engineering of the host cell line, as well as the culture conditions including media composition, shape the protein functionality ${ }^{7,45}$ and effect undesired by-products, such as aggregates ${ }^{46}$ and low-molecular-weight species (LMW) ${ }^{47}$. The choice of the host cell is decisive, since each of the frequently used cell lines (CHO, NS0 and SP2/0) has a cell-line specific glycosylation fingerprint. Depending on the desired recombinant protein quality, one or the other host must be chosen. The quality may be further fine-tuned, using gene-knock-out and gene-over-expression technologies. Specifically, the glyco-engineering 
technology has greatly advanced recently, and as a result, clinical trials of more than 15 glycoengineered antibodies have been performed ${ }^{48}$. In addition to the host cell genome, the cell culture process conditions assuring cell growth and protein expression shape the protein quality attributes as well. According to the common phrase 'the process is the product', the metabolism of the cells closely depends on the culture conditions, including the $\mathrm{pH}^{17}$, the temperature ${ }^{49}$, the oxygen tension ${ }^{17}$, the $\mathrm{CO}_{2}$ content in the culture broth ${ }^{50}$, as well as the type of process, namely perfusion or fed-batch mode ${ }^{31}$. Different metabolic states, which result from differences in culture parameters, very likely express proteins with altered quality attributes. Many authors have published extensive reviews, depicting the current state of the art and strategies, which focus on the cell line ${ }^{1,7,16,45}$ and the cell culture parameters ${ }^{18,51}$ Furthermore, through the concentration adjustment of selected media components, and in some cases by supplementing the medium with specific co-factors, it is possible to adjust the glycosylation profile ${ }^{52}$, the charge variants ${ }^{53}$, the aggregation level ${ }^{46,54}$, and the abundance of LMW species ${ }^{55}$. Figure 1.1 outlines the three main strategies allowing to affect cell culture process performance and to tailor the quality attributes of therapeutic molecules.

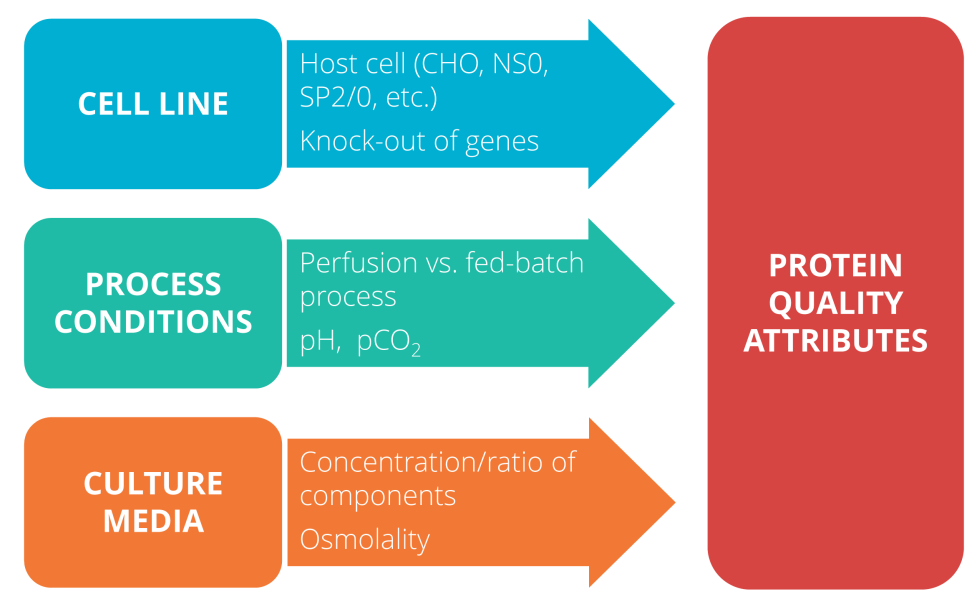

Figure 1.1 - Parameters affecting process performance and recombinant protein quality attributes. A non-exhaustive list of examples for each parameter category is presented. 



\section{Chapter 2}

\section{The Potential of Media to Enhance Protein Quality}

Cell culture media design has a great potential to modulate the key quality attributes of the molecule ${ }^{25}$, and in particular by means of high-throughput design of experiment (DoE) approaches ${ }^{26,27}$, due to its central role in the upstream manufacturing ${ }^{19}$. The environment the cell is cultured in closely influences its metabolism to a great extent ${ }^{20-22}$. Given its potential, protein quality tuning by media design gained much interest in the last few years. Crowell et al. ${ }^{23}$ described in 2007 how manganese supplementation modulated the glycosylation state of erythropoeitin (EPO) in a CHO cell culture. These findings are based on early experiments by Kaufman et al. who observed the pivotal role of manganese in the secretory pathway of complex $\mathrm{N}$-linked and $\mathrm{O}$-linked oligosaccharides, thus suggesting the unique requirement for manganese ${ }^{56}$. With respect to cell-line engineering and process modifications, we think media design is a particularly powerful approach since it can be rapidly implemented for any selected production cell line. Nonetheless, due to the complexity of post-translational modifications each protein has to be considered on a case-by-case basis. Hence, to cope with the uniqueness of each protein structure and cell-line specificities in the development of a new recombinant therapeutic it will be most appropriate to create a media component library. When the need to adjust the protein quality arises, one can specifically select one or several compounds from this library in order to fine-tune the quality profile. Apart from the knowledge and the ability to fine-tune quality attributes, sophisticated analytical assays are of great importance for the design of tomorrow's drugs to pick up on the variations induced by media design. Over time, analytical assays have been further developed and particularly refined for biosimilars to compare their quality with respect to the originator molecule's fingerprint.

Since the introduction of the classical cell culture media formulation designed by Eagle and $\mathrm{Ham}^{57}$, chemically defined media compositions used in mammalian cell culture have undergone great enhancements, while keeping most of the initial nutrient categories, consisting of amino acids, vitamins, salts, and glucose. Most if not all media optimization efforts of 
both industry and academia rely on trial and error approaches. Several iterations of media improvement may sometimes be required. They accordingly entail intensive development workloads and durations.

Hereafter, we provide an extensive overview of the way both common cell culture media components and new supplements-either tested in cell culture or in enzyme assays and other contexts-including their interactions affect the major quality attributes of recombinant therapeutic proteins expressed in mammalian cell culture. The quality attributes include glycosylation, charge variants, aggregates, LMW species, and misincorporation of amino acids in the protein backbone. Where available, the cell line and the tested concentration ranges are outlined. Moreover, special attention will be given to either specific or potent inhibitors and activators of glycosylation processing steps.

\subsection{Glycosylation}

Among the various post-translational modifications, glycosylation, the synthesis as well as the attachment and processing of oligosaccharide side chains of a polypeptide ${ }^{58}$, has the most significant impact on pharmacokinetics and protein physicochemical characteristics ${ }^{14}$. Two main types of glycosylation exist ${ }^{59}$ : asparagine-linked glycosylation, also called $N$-linked glycosylation and serine/threonine- $O$-linked glycosylation. We are interested in the asparaginelinked glycosylation, which is by far the most frequent in monoclonal IgG antibodies ${ }^{60}$. In these biotherapeutic molecules, $\mathrm{N}$-glycans are linked to the two conserved asparagine residues (Asn 297) in the $\mathrm{CH} 2$ domain of the Fc region ${ }^{61}$. A large number of reports describe various impacts of glycosylation on the quality attributes of biologics including in vivo efficacy ${ }^{62-64}$, pharmacokinetics (PK) ${ }^{11}$, antibody-dependent cellular cytotoxicity (ADCC) ${ }^{63,65}$ and complementdependent cytotoxicity (CDC) activities ${ }^{66}$, stability and overall structure of the molecule ${ }^{63}$, clearance and half-life in vivo ${ }^{10}$ as well as immunogenicity ${ }^{10,67}$. Non-fucosylated therapeutic antibodies exhibit 50 to 1,000-fold higher efficacy than their fucosylated counterparts ${ }^{65}$ due, in most cases, to enhanced ADCC activity ${ }^{68}$. Given the abundance of research efforts in this area, it is no surprise that many have concluded that glycosylation is one of the main areas requiring development ${ }^{69}$ to improve efficacy ${ }^{70}$ and safety ${ }^{10,70}$ of next generation therapeutics ${ }^{61}$. Hence, the control of glycosylation of recombinant therapeutic molecules expressed in non-human systems is decisive ${ }^{71}$.

As depicted in figure 2.1, glycosylation takes place in the endoplasmic reticulum (ER) where the oligosaccharide chain is attached to the protein backbone and subsequently trimmed to form oligomannose species by a series of enzymatic reactions. In mammalian cells, the glycoprotein undergoes further processing in the Golgi apparatus, yielding first of all mannose 5 (Man5), then hybrid, and eventually complex glycans ${ }^{18,63}$. The cell culture conditions including culture media components, the availability of the nucleotide sugar substrates, and the expression levels of the enzymes involved in the attachment and transformation of carbohydrate structures, 
define the level of antennarity and sialylation ${ }^{18}$.

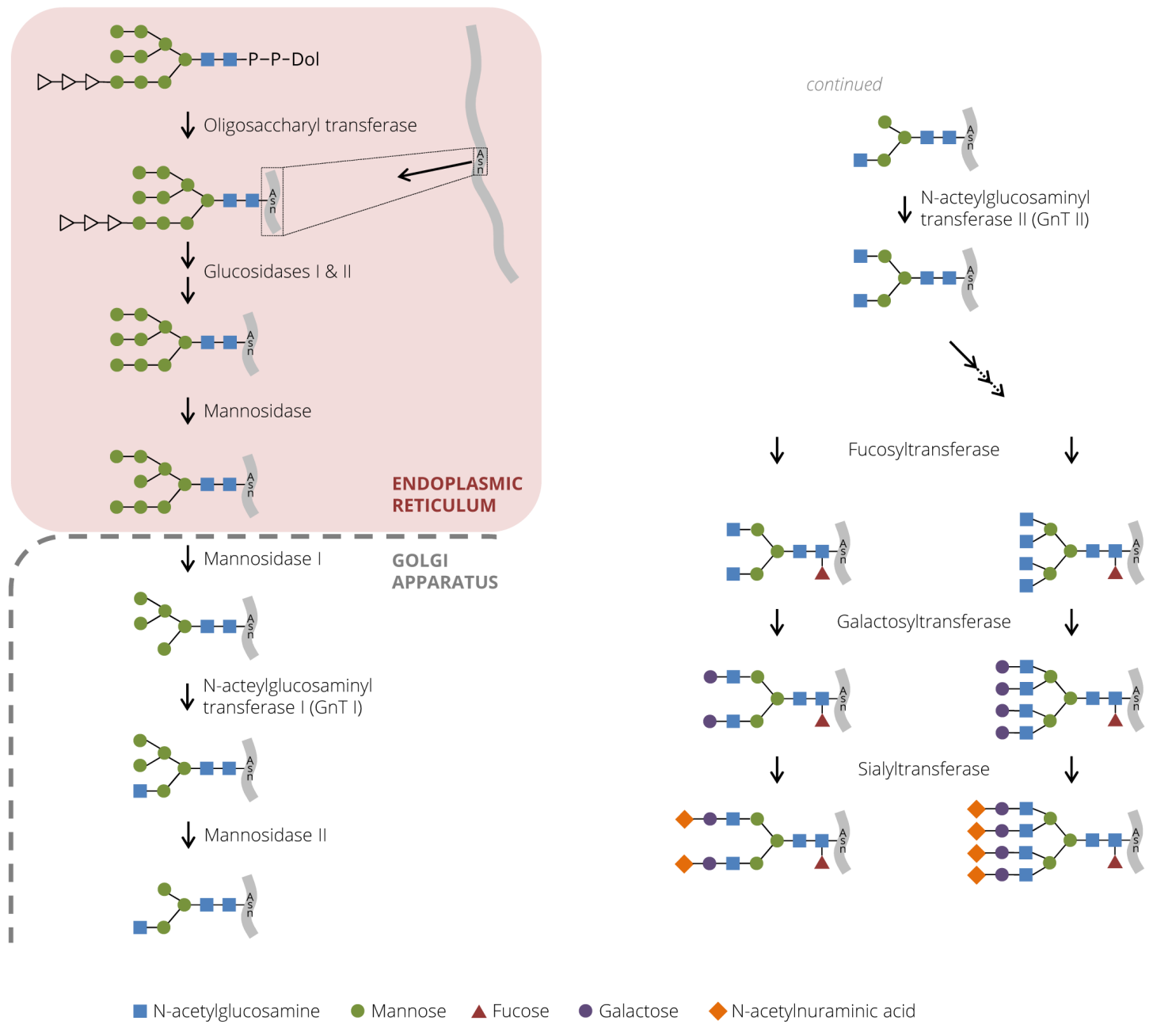

Figure 2.1 - Schematic N-glycosylation pathway in the endoplasmic reticulum (ER) and Golgi apparatus.

Nonenzymatic glycosylation, called glycation, occurs naturally in the human body and can also take place during cell culture ${ }^{72}$. Glycation is the result of a condensation reaction, also termed Maillard reaction, between free protein amine groups and a reducing sugar, resulting in unstable Schiff bases ${ }^{73}$. Subsequent spontaneous oxidation or rearrangement leads to the more stable Amadori products, which are eventually transformed through a series of intermediates into advanced glycation end products (AGE) ${ }^{74}$. Even though one has not observed a specific sequence of the positively charged primary amines located on the protein structure's surface, which increases the likelyhood of glycation, local environments that contain histidine residues or basic residues seem to favor glycation in structurally known proteins ${ }^{75}$. This posttranslational chemical reaction will also be discussed, as it is reported to potentially affect the biological activity (increased or decreased) as well as $\mathrm{PK}^{76}$. 
Thus, the strategies to tweak post-translational modifications by media design are of paramount importance to engineer tomorrow's therapeutic molecules. Due to the exceedingly high number of articles addressing the subject of glycosylation, an extensive presentation of all glycosylation related aspects would go beyond the scope of this review. Rather, with the applicability in a manufacturing environment in mind, we will concentrate on promising supplements that have a potential to modulate fucosylation, galactosylation, and sialylation as well as the levels of high mannose species. A highly promising approach is the use of inhibitors, targeting specifically the enzyme of interest, and other potent inhibitors or co-factors, which are not necessarily specific to one glycosylation processing step. A selection of those we consider the most relevant are presented in table 2.1, and are further discussed along other compounds affecting glycosylation in the corresponding subsection.

Table 2.1 - Specific and/or potent inhibitors, activators of glycosylation processing steps as well as compounds increasing the availability of the precursor.

\begin{tabular}{lll}
\hline Component & Effect & Specificity \\
\hline Kifunensine & Inhibition of mannosidase I & Yes \\
Mannostatin & Inhibition of mannosidase II & Yes \\
Fluorinated fucose analogs & Inhibition of fucosyltransferase & Yes \\
Cibracon Blue 3GA & Inhibition of fucosyltransferase & Yes \\
Reactive Red 120 & Inhibition of fucosyltransferase & Yes \\
2-naphthyl-2-butanamido- & Inhibition of galactosyltransferase & Yes \\
2-deoxy-1-thio- $\beta$-D-gluco- & & \\
pyranoside & & No \\
Galactose & Favors galactosylation and indirectly & No \\
& sialic acid & Yes \\
Fluorinated sialic acid analogs & Inhibition of sialyltransferase & No \\
Manganese & Affects the levels of various oligosaccha- & No \\
& ride species & \\
\hline
\end{tabular}

\subsubsection{Non Specific Impact of Media Components}

While the limitation of glucose in the culture medium allows to reduce lactate production ${ }^{77}$, it has been observed that critical limitation of the former leads to glycosylation heterogeneity ${ }^{78}$, due to a decreased UDP-acetylglucosamine (UDP-GlcNAc) availability ${ }^{79}$. The absence of glucose affects the synthesis of oligosaccharide precursors, as glucose-starved cells alter its synthesis process ${ }^{80}$. In a CHO cell culture test it was shown that the proportion of nonglycosylated antibody was correlated to the duration the cells were deprived of glucose: the absence of glucose during 24 hours led to $45 \%$ of non-glycosylated $\mathrm{mAb}^{81}$. In another study with the human cell line rF2N78 in fed-batch culture it was observed that in the absence of glucose in the feed about $44 \%$ of the product was aglycosylated, while no aglycosylated antibody was expressed when feeding glucose throughout the culture ${ }^{82}$. In glucose-depleted 
CHO (DUXB) batch cultures producing a chimeric human-llama monoclonal antibody up to $51 \%$ non-gycosylated forms were obtained. In addition to reduced site occupancy, glucose depletion also decreased the galactosylation index (GI) at the maximal observed difference from 0.65 to 0.26 , and sialylation by $85 \% 83$.

Glucose and glutamine (Gln) concentrations below $1 \mathrm{mM}$ were reported to be detrimental to glycosylation or, if desired, to allow the production of non-glycosylated molecules ${ }^{62,84,85}$. In a continuous culture with BHK-21 cells at $<0.5 \mathrm{mM}$ glucose or $<0.2 \mathrm{mM}$ glutamine exclusively neutral diantennary oligosaccharides with or without core $\alpha 1$-6-linked fucose were present that held no, one or two $\beta 1$-4-linked galactose ${ }^{84}$. Likewise, interferon- $\gamma$ (IFN- $\gamma$ ) expressing CHO cells cultured in a chemostat under glucose limitation yielded a lower proportion of fully glycosylated protein ${ }^{85}$. IFN- $\gamma$ expressing CHO cells in fed-batch mode at low glutamine $(<0.1 \mathrm{mM})$ or glucose $(<0.70 \mathrm{mM})$ concentrations resulted in decreased sialylation and increased presence of minor glycan species consisting of hybrid and high-mannose types ${ }^{62}$.

Manganese plays a fundamental role in the glycosylation pathway, as shown by many authors ${ }^{23,52,56}$. It determines the levels of various oligosaccharide species including high mannose, galactosylated, fucosylated and sialylated proteins. As cofactor of many enzymes, manganese modulates the glycosylation profile and it was noted that a lack of this transition metal inhibits O-linked glycosylation ${ }^{23}$. Its specific role in each glycosylation processing step is described more thoroughly in the following subsections.

The modulation of the glycosylation level can be obtained through media complementation with precursors and/or cofactors, aiming to reduce the formation of ammonium by limiting the amino acid concentrations in the medium ${ }^{25}$. It has also been shown that increased substrate levels, in particular, nucleotide-sugar precursors including UDP-Hex, UDP-HexNAc, and CMP-sialic acid induced an overall increase of the glycosylation flux ${ }^{86}$.

\subsubsection{High Mannose Species}

In the Golgi apparatus the $\alpha$-mannosidase I enzyme trims the high mannose species generated in the ER, yielding Man $5^{18}$. One of the strategies to increase the level of proteins equipped with a high mannose oligosaccharide chain consists in inhibiting the $\alpha$-mannosidase I as can be done efficiently with kifunensine, an alkaloid originally isolated from Kitasatosporia kifunense, an actinobacterium ${ }^{87}$. It has been known for years for its potent inhibition abilities of $\alpha$-mannosidase I. A study with different types of plant mannosidases exhibited efficient inhibition by kifunensine of mannosidase I with a half maximal inhibitory concentration ( $\mathrm{IC}_{50}$ ) of $0.2-0.5 \mu \mathrm{M}$, but not of mannosidase II, indicating that kifunensine only inhibits mannosidase I in the Golgi apparatus and does not affect the corresponding enzyme in the $\mathrm{ER}^{88}$. In a more recent experiment in the plant Arabidopsis thaliana it was found that the amino acid sequences of human Golgi- $\alpha$-mannosidases were closely related to the enzyme of the plant, and the measured kifunensine inhibition, $\mathrm{IC}_{50}$, amounted to $0.30-0.47 \mu \mathrm{M}^{89}$. 
Other authors concluded that kifunensine had affected ER mannosidase in their experimental conditions ${ }^{90}$. Different $\mathrm{IC}_{50}$ of the latter are mentioned in the literature. For example, IgG antibody expressing hybridoma cells generated exclusively oligomannose structures (Man9 to Man5) if the cells were exposed to $80-100 \mathrm{ng} / \mathrm{mL}$ kifunensine throughout the culture. At intermediate kifunensine concentrations $(40-60 \mathrm{ng} / \mathrm{mL})$, oligomannose abundance settled in the range of 69 to $90 \%$ and decreased to about $18 \%$ at $20 \mathrm{ng} / \mathrm{mL}$ of kifunensine ${ }^{91}$. It is not surprising that they concluded that kifunensine is effective in producing antibodies with oligomannose-type glycans from $\mathrm{CHO}$ cells, as no impact on both titer and viability but only on maximum viable cell density was observed. These results also show the potential to effectively fine-tune the ratio of oligomannose and hybrid glycans by varying the kifunensine concentration of the culture medium. Hence, kifunensine has become the most popular $\alpha$-mannosidase I inhibitor ${ }^{92}$.

The presence of 1-deoxymannojirimycin, another specific mannosidase inhibitor from the class of 1-deoxyazasugars, resulted in an important increase of the half maximal inhibitory concentrations compared to kifunensine ( $\mathrm{IC}_{50}$ of $\left.30-40 \mu \mathrm{M}\right)^{89}$. In another study, the inhibition coefficient of jack bean $\alpha$-mannosidase by 1-deoxymannojirimycin was even higher: $\mathrm{IC}_{50}=840 \mu \mathrm{M}^{93}$. Polyhydroxy substituted piperidine derivatives strongly and competitively inhibited the enzyme activity in a study of class II $\alpha$-mannosidase from Aspergillus fischeri. The benzyl group containing compounds of this class exhibited greater inhibition capabilities (IC $\mathrm{I}_{50}$ of 31 to $51 \mu \mathrm{M}$ ) than the molecule closely related to 1-deoxymannojirimycin without benzyl group ( $\mathrm{IC}_{50}$ of $237 \mu \mathrm{M}$ ) ${ }^{94}$. Among the wide range of biological activities such as antibiotic, antitumor, DNA-binding properties and growth-regulating effects in plants, gabosines (natural carbasugars) inhibit glycosidases. (-)-Gabosine J inhibits jack bean $\alpha$-mannosidase with an IC $_{50}$ of $260 \mu \mathrm{M}$, however no biological activity of its enantiomer, (+)-gabosine J, is reported ${ }^{95}$. $N$-butyl-azepane derivatives displayed a remarkable selectivity for the inhibition of cytosolic $\alpha$-mannosidase in both HL60 and MDBK cells cultured in RPMI 1640 medium and led to a drastic increase of high mannose species at a concentration of $100 \mu \mathrm{M}$, showing substantial inhibition capability of these compounds ${ }^{90}$. More recently, the role of a plant-derived alkaloid called calystegine $\mathrm{B}_{3}$ was studied both in vitro and in vivo. A substantial structure change and increase of free oligosaccharides in the cytosol was observed with no effect on cell-surface oligosaccharides, resulting in the assumption that calystegine $\mathrm{B}_{3}$ specifically inhibits cytoplasmic $\alpha$-mannosidase. While in vitro the $\mathrm{IC}_{50}$ value for cytoplasmic $\alpha$-mannosidase was low $(8.7 \mu \mathrm{M})$, in vivo inhibition was much weaker, requiring $1 \mathrm{mM}$ calystegine $\mathrm{B}_{3}$, which might be due to inefficient incorporation of this compound according to the researchers ${ }^{96}$. Inhibitory effects of plant extracts were also found ${ }^{97}$. Their mode of action may be interesting for further investigations, in order to identify single, chemically defined inhibitors of $\alpha$-mannosidase. In a screening exercise of the $\alpha$-mannosidase family, it was found that despite partial reactivation due to divalent metals, EDTA inactivated all enzymes, and the addition of $\mathrm{Ca}^{2+}$ allowed to recover the complete enzyme activity ${ }^{98}$. This observation highlights the pivotal role of $\mathrm{Ca}^{2+}$ in the catalytic cleavage of $\alpha-1,2$ bonds ${ }^{98}$. In addition to kifunensine other inhibitors such as swainsonine and mannoimidazole inhibit mannosidases as well ${ }^{98,99}$. Divalent transi- 
tion metal ions including $\mathrm{Cu}^{2+}$ and $\mathrm{Se}^{2+}$ displayed noncompetitive inhibition $\left(\mathrm{K}_{i, C u}=22 \mathrm{nM}\right.$, $\left.\mathrm{K}_{i, S e}=28 \mu \mathrm{M}\right)$, whereas $\mathrm{Co}^{2+}$ showed competitive inhibition $\left(\mathrm{K}_{i}=1.2 \mathrm{mM}\right)$. Further tests by the same researchers revealed inhibitory effects of $\mathrm{Pb}^{2+}$ and $\mathrm{Hg}^{2+} 94$.

Mannostatin A, a reversible and competitive inhibitor of Golgi $\alpha$-mannosidase II, belongs to the most potent inhibitors of this enzyme ${ }^{100,101}$. Zinc plays an important catalytic role in the hydrolyzation step involving $\alpha$-mannosidase II, as it helps to stabilize the transition state by relieving the electron deficiency of the Michaelis complex ${ }^{102}$. On the opposite, another study concluded that class II $\alpha$-mannosidase is neither metal ion dependent nor inactivated by EDTA $^{94}$. In a more recent article focusing on the $\alpha$-mannosidase activity in stallion epididymal fluid and spermatozoa, $\mathrm{Zn}^{2+}$ triggered the enzyme activity in acidic conditions, while the neutral form was stimulated by $\mathrm{Co}^{2+}$. It was also observed that the acidic form was sensitive to swainsonine, a potent inhibitor of the class I enzyme ${ }^{103}$. The inhibitory effect of swainsonine on the class II enzyme had been described previously ${ }^{89}$. The bicyclic derivative of 1-deoxymannojirimycin, castanospermine, an indolizine alkaloid isolated from the seeds of the Australian chest nut tree Castanosperum australe ${ }^{104}$, inhibits the ER glucosidase enzymes, and as a result, further glycan processing ${ }^{105}$.

In an attempt to control the antibody glycosylation with respect to cell culture conditions, a more than twofold increase from 12 to $28 \%$ of Man 5 was obtained by synergistic effects of media and feed osmolality as well as culture duration. However, the mechanisms of the impact of osmolality on the enzyme activity are not fully understood and remain to be studied more thoroughly. In the same experiment, the level of Man5 glycans significantly decreased due to the supplementation of $0.25-1.0 \mu \mathrm{M}$ manganese chloride $\left(\mathrm{MnCl}_{2}\right)$ at both 300 and $400 \mathrm{mOsm} / \mathrm{kg}^{63}$. Another group reported that $\mathrm{MnCl}_{2}(0.04 \mathrm{mM})$, galactose $(100 \mathrm{mM})$ and/or $\mathrm{NH}_{4} \mathrm{Cl}(10 \mathrm{mM})$ increase Man5 species ${ }^{106}$. A recent study showed as well that manganese increases high mannoses on mAb produced in $\mathrm{CHO}$; however in glucose limiting or absent conditions ${ }^{107}$.

A substantial augmentation of high mannose species was achieved with supplementation of uncommonly used sugars, where sucrose addition to the cell culture medium led to a $37 \%$ higher amount of mannosylated N-glycans with no influence on growth nor productivity. The presence of tagatose, a monosaccharide resembling galactose, resulted in an increase as well. It was lower, however, compared to sucrose, and reduced the titer at high concentrations. As sucrose is composed of fructose and glucose, one would expect to see a change in the glycosylation profile if they are present independently. Interestingly, the oligomannose level did not change if the medium contained glucose and fructose instead of sucrose. The authors hypothesize that sucrose and tagatose may inhibit the formation of the UDP-GlcNAc nucleotide-sugar by constricting the supply of the uridine diphosphate N-acetylglucosamine (UDP-GlcNAc) substrate. Lower substrate levels, they say, lead to reduced enzymatic reaction rates ${ }^{108}$. This behavior is not surprising since the opposite is the case if the medium is supplemented with nucleotide-sugar precursors. Precursor supplementation allows to favor the corresponding glycan species $^{86}$. 
In perfusion cultures with serum-free adapted DXB-11 CHO cell lines expressing different antibodies, it was presented that the use of mannose as a carbon source entailed a more than 2fold increase of high mannose glycoforms ${ }^{109}$. Their results show that mannose may substitute glucose as a carbon source. At the highest tested mannose/glucose ratio (0.94), a significant increase of Man8, Man7, Man6 and Man5 was observed. The latter was the major species in all study conditions. The researches hypothesized that three pathways, including GDP-mannose biosynthesis, early protein glycosylation and UDP- N-acetylglucosamine biosynthesis, might take part in the observed increase. The same research organization further explored the metabolism of mannose and the mechanism for increased utilization of mannose, applying ${ }^{13} \mathrm{C}$ flux analysis. A greater carbon flux in the GD-mannose synthetic pathway effected more abundant intracellular concentration of mannose-containing metabolites ${ }^{110}$.

Another report presents the decrease of high mannose species in semi-continuous 24-deepwell-plate cultures of various $\mathrm{CHO}$ cell lines following cell cycle arrest. The strategy consisted in adding a small molecule, which directly targeted the cell cycle G1-checkpoint. As a result the proportion of high mannose species decreased in the tested cell lines with respect to the control, and galactosylated species became more abundant ${ }^{49}$.

\subsubsection{Fucosylation}

In an in-depth overview on various fucosyltransferase (FucT) inhibitors the authors explain that each type of this enzyme class catalyzes the transfer of fucose to either terminal or core oligosaccharide positions according to its distinct substrate specificity and site of action ${ }^{111}$. A particular interest is drawn to the inhibitors of FucT-VIII, as it is responsible for the $\alpha-1,6$ linkage of fucose on the core of $\mathrm{N}$-glycans in mammalian cell culture.

As part of a recent development of cell-permeable and family-specific inhibitors of fucosyltransferase, the inhibitory capacity of fluorinated fucose analogs was discovered by testing in cells. The strategy consisted in attaching a fluorine atom proximal to the endocyclic oxygen, as these compounds are readily converted to the corresponding donor substrate analogs within the cell. The 2-fluor-fucose analog substantially reduced core fucosylation of N-linked glycans in CHO cells at concentrations of about 30-500 $\mu \mathrm{M}$, thus revealing its inhibitory effect on FucT-VIII ${ }^{112}$. Likewise, the non-sugar related compounds, Cibracon Blue 3GA $\left(\mathrm{K}_{i}=11 \mu \mathrm{M}\right)$ and Reactive Red $120\left(\mathrm{~K}_{i}=2 \mu \mathrm{M}\right)$ specifically inhibit FucT-VIII ${ }^{113}$. With the objectif to avoid incorporation of non-native sugar, which is an important feature in biosimilar development, a fucose-1-phosphonate analog, fucostatin II, was found to inhibit fucosylation in CHO cell cultures when added into the medium at 5 to $100 \mu \mathrm{M}$, while no detectable incorporation of non-native entities was found among the antibody glycans ${ }^{114}$.

High-throughput quantitative MALDI-TOFMS-based screening identified an azidosugar nucleotide derivative as being a specific FucT-VIII inhibitor ${ }^{115}$. However, the high negative charge of nucleotide sugar analogs prevents them from efficiently crossing cell membranes and, with 
a few exceptions, questions their utility in cell culture ${ }^{112}$. Much simpler in structure, gallic acid and its derivatives efficiently inhibit FucT-VII in the presence of $10-15 \mathrm{mM} \mathrm{Mn}^{2+116}$. In a $\mathrm{CHO}$ batch shake flask culture, lower fucosylation was observed with decreasing glutamine concentration (range: $0-8 \mathrm{mM}$ ), and it is assumed that the reduction in the glycolytic flux due to glutamine limitations impacts glycosylation. The same authors report no significant glycan differences in continuous culture between two steady states by decreasing the Gln concentration from 8 to $0 \mathrm{mM}$. Nonetheless, between additional steady states (steady state 3: $8 \mathrm{mM}$ Gln, steady state 4: $0 \mathrm{mM} \mathrm{Gln}$ ) a significant glycan species distribution occurred thus showing the impact of the metabolic flux on glycosylation ${ }^{117}$. Following the addition of sucrose, which led to higher levels of high mannose glycans (cf. section 2.1.2), afucosylated proteins became indirectly more frequent ${ }^{108}$. Despite the above described inhibitors, it seems that some authors believe that both specific and potent inhibitors for FucT-VIII have yet to be identified ${ }^{118}$. Mycophenolic acid supplementation was successfully used to increase the level of afucosylated glycans in three CHO cell lines-CHO-DG44 and CHO-DXB11 expressing the same $\mathrm{mAb}$, and CHO-S expressing a fusion protein - which influenced the GTP synthesis and in one cell line (CHO-DXB11) even directly inhibited the FUT8 expression levels ${ }^{119}$.

Fucosyltransferases and sialyltransferases may compete for the same acceptor substrates, which was demonstrated with $\mathrm{N}$-acetyllactosamine (LacNAc) derivatives ${ }^{120}$. As a result, selective inhibition of sialic acid addition by adding a fluorinated sialic acid analog increases fucosylation ${ }^{112}$. Due to its effective inhibition of steps occurring prior to fucose attachment, kifunensine effectively retains oligomannose residues and hence allows to express non fucosylated proteins at concentrations beyond $60 \mathrm{ng} / \mathrm{mL}^{91}$. It is important to take into account the existence of a mannosyl-glycoprotein- $\mathrm{N}$-acetylglucosaminyltransferase I-independent (GnT I) fucosylation pathway, as described by various authors when modulating the glycosylation levels, since inhibition of GnT does not necessarily prevent further processing of the oligosaccharide chain including fucosylation. The same authors say that the presence of fucosylated high mannose species in combination with no GnT I activity and hybrid-type structures can be observed in CHO cells ${ }^{121}$.

\subsubsection{Galactosylation}

Many authors have described the effect of manganese $\left(\mathrm{Mn}^{2+}\right)$, a cofactor for $\beta 4$-GalT1, on the level of both $\mathrm{N}$ - and O-linked glycosylation site occupancy. Cultures performed at higher $\mathrm{Mn}^{2+}$ concentrations $(40 \mu \mathrm{M})$ exhibited increased galactosylation and higher sialylation of recombinant human erythropoietin (EPO), however, the product yield was greatly reduced ${ }^{23}$. A decrease of Man5 and hence higher abundance of galactosylated species were observed at increasing $\mathrm{Mn}^{2+}$ concentration: $0.25-1.0 \mu \mathrm{M}^{63}$. A recent DoE experiment with post-seeding supplementation of 0-40 $\mu \mathrm{M} \mathrm{Mn}^{2+}, 0-20 \mu \mathrm{M}$ uridine (Urd) and 0-100 $\mu \mathrm{M}$ galactose (Gal) led to the conclusion that $\mathrm{Mn}^{2+}$ was necessary but not sufficient to improve galactosylation, and that synergistic combinations of Urd and Gal maximized galactosylation ${ }^{122}$. The resulting osmolality increase at high galactose concentrations tested may however be toxic to the cells 
and hence harm the culture. In a fed-batch culture, both the level of galactosylation and the distribution of galactosylated glycoforms (G0F, G1F and G2F) were influenced by feeding $\mathrm{Mn}^{2+}$, Urd and Gal at concentrations from 0 to $20 \times \mathrm{Mn}^{2+}$-Urd-Gal $\left(1 \times \mathrm{Mn}^{2+}: 0.002 \mathrm{mM}\right.$, $1 \times$ Urd: $1 \mathrm{mM}, 1 \times$ Gal: $5 \mathrm{mM}$ ). Galactosylation increased from 3 to $23 \%$ for one cell line and from 5 to $29 \%$ for a second one, mainly due to a shift from G0F to G1F, and to a lower extent, to increases in G2 and G2F. In these conditions only minor modifications of other glycoforms or quality attributes were induced ${ }^{52}$. In the absence of synergy, the impact of the galactose concentration on increased galactosylation is limited ${ }^{123}$. More recent results however displayed an increase of galactosylation from 14 to $25 \%$ of monoclonal antibodies expressed in CHO DG44-derived fed-batch suspension cell cultures, supplementing medium and feeds to reach a final media concentration of $20 \mathrm{mM}$ of galactose ${ }^{124}$. In the same study, $20 \mathrm{mM}$ GlcNAc addition effected a $4 \%$ decrease of galactosylated entities. In another test, the asparagine concentration influenced the distribution of G0F, G1F and G2F, likely due to altered enzyme activity caused by increased intracellular $\mathrm{pH}$, where higher G0F and lower G1F and G2F emerged with increasing asparagine levels (supplementation of 0-10 mM Asn) ${ }^{125}$.

On the other hand, inhibitors of galactosyltransferase (GalT) will lead to decreased galactosylation of the protein backbone. It was observed that elevated ammonium levels ( $\geq 10 \mathrm{mM}$ ) during the cell culture reduced both GalT gene expression and activity. Furthermore, these conditions significantly impacted the post-glycosylation process in the Golgi apparatus, while it had less impact on ER and cytosol compartments ${ }^{126}$. Structural analogs and mimics of the natural sugar-nucleotide UDP-galactose (UDP-Gal) have a great potential to specifically inhibit GalT. An UDP-Gal derived compound, bearing an additional substituent at the 5-position of the uracil base has been described as being a new type of GalT inhibitors $\left(\mathrm{K}_{i}=426 \mu \mathrm{M}\right)^{127}$. It was demonstrated that the GalT inhibition by 5 -substituted UDP-Gal derivatives is broadly applicable to this enzyme class, and that despite of their polarity they are taken up by HL-60 cells $^{128}$. Deoxygenated disaccharide analogs (per- $O$-acetylated GlcNAc $\beta 1$-3Gal $\beta$ - $O$-naphthalenemethanol and C-3' and C-4' hydroxyl-modified analogs) competitively inhibited $\beta 4$ GalT1, or related GalT enzymes in tumor cells in the tested concentration range: 0-50 $\mu \mathrm{M}^{129}$. Conjugates of 2,4-diamino sugars and uridine also inhibit GalT at $1 \mathrm{mM}$. Their inhibitory capacity seems to depend on conformational flexibility and thus on the chelating abilities of the hinge-like diamino sugar towards a metal ion such as $\mathrm{Mn}^{2+}$ within the structure of the enzyme ${ }^{130}$. Inhibition of mammalian $\beta 4$-GalT1 and $\beta 3$-GalT5 can also be obtained by the use of bivalent imidazolium salts up to $1 \mathrm{mM}$, which are not substrate analogues and can therefore inhibit other types of GalT too. Cell membranes incorporate these compounds due to their detergent-like properties, but it has yet to be elucidated whether they are capable to cross the membrane to eventually reach the Golgi apparatus, and inhibit GalT in vivo ${ }^{131}$. 2-naphthyl2-butanamido-2-deoxy-1-thio- $\beta$-D-glucopyranoside specifically and strongly inhibited $\beta 4$ GalT1 at a concentration of $0.5 \mathrm{mM}$ in human and mouse cell homogenates and bovine serum (FBS), while it does not affect the activity of related enzymes ${ }^{132}$. The use of peracetylated fluorosugars, which successfully inhibited both fucosylation and sialylation, is suggested as UDP-galactose analogs to inhibit GalT ${ }^{112}$. The inhibition constant of UDP-2FGal was found to 
be $149 \mu \mathrm{M}^{133}$.

\subsubsection{Sialylation}

Manganese $\left(\mathrm{Mn}^{2+}\right)$ favors sialylation according to a publication describing a correlation between the enhancement of sialylated G1 $\mathrm{N}$-glycans and the supplementation of $\mathrm{Mn}^{2+}$ $(0-40 \mu \mathrm{M})$ in the presence of Urd and Gal during the culture ${ }^{122}$. While improved sialylation of IFN- $\gamma$ was the result of medium supplementation with the specific sialic acid precursor $\mathrm{N}$-acetylmannosamine (ManNAc) at $20 \mathrm{mM}$ in a CHO cell line (cotransfected with genes for dihydrofolate reductase $)^{134}$, it remained unchanged for human tissue inhibitor of metalloproteinases 1 (TIMP-1) at the same ManNAc concentration in GS-NS0 and GS-CHO cells ${ }^{135}$. Likewise, feeding CHO-K1 cells producing EPO with 10 mM ManNAc increased sialylation ${ }^{136}$. Unlike peracetylated ManNAc, ManNAc does not readily cross the cell membrane, and hence rather high concentrations of the latter are required ${ }^{137}$. Consequently, its adoption at large scale is questioned by the inefficient metabolic utilization, but due to the development of acetylated ManNAc analogs, which are metabolized up to 900 -fold more efficiently than their natural counterparts ${ }^{138}$, this strategy remains interesting to modulate sialylation. At a concentration range of $1-10 \%(\mathrm{v} / \mathrm{v})$, glycerol (1,2,3-propanetriol) displayed an enhancing effect on the level of sialylation in IFN- $\beta$ batch cultures ${ }^{139}$. Fluorinated sialic acid analogs readily cross the cell membrane and do not negatively impact cell growth and viability. They are therefore potent SiaT inhibitors beyond $30 \mu \mathrm{M}$. The axial 3-fluor- $N$-acetyl-neuramic acid $\left(3 \mathrm{~F}_{a x}\right.$-Neu5Ac) extensively inhibits SiaT in vivo. The orientation of the fluor atom is pivotal, as the equatorial analog $\left(3 \mathrm{~F}_{e q}\right.$-Neu5Ac) has no inhibitory effect ${ }^{112}$. In a fed-batch CHO culture expressing a Fc-fusion protein, the addition of 1-50 $\mathrm{mg} / \mathrm{L}$ of hydrocortisone increased the proportion of sialic acid moieties up to a level 2.5 times higher than the control, which corresponds to a 5 -fold increase of acidic isoforms ${ }^{64}$.

Interestingly, galactose appears to substantially augment sialic acid content, presumably as a result of an increase in readily available terminal galactose moieties needed by sialyltransferases (SiaT) as acceptors for sialic acid addition to terminal N-glycans. As a nonspecific component, galactose also influences other glycosylation steps including galactosylation ${ }^{122}$. This finding was further confirmed in GS-CHO cell line expressing the human tumor necrosis factor receptor linked to the Fc portion of human IgG1. The addition of $20 \mathrm{mM}$ of galactose in 2-L bench scale and 200-L pilot scale fed-batch cultures led to $20.3 \%$ increase of sialylated glycans ${ }^{140}$.

Whereas in many cases an increased sialylation is desired for a greater half-life of the protein in vivo, it may be required, for instance, to reduce its level to better mimic the originator molecule in the frame of biosimilar development. Ammonium levels not only inhibit galactosylation, but also have an impact on the abundance of sialylated species, due to the sparsity of galactosylated glycoforms and limited expression levels of $\alpha$-2,3-sialyltransferase (SiaT) ${ }^{126}$. In contrast to human IgG, sialic acids are attached to the terminal galactose residues of antibodies 
expressed in CHO cells via an $\alpha-2,3$ linkage exclusively ${ }^{68}$. It was reported that by reducing glutamine concentrations to $0 \mathrm{mM}$ in $\mathrm{CHO}-\mathrm{K} 1$ batch and perfusion cultures, sialylation was inhibited, thus leading to more abundant neutral N-linked glycans ${ }^{117}$. Dimethyl sulfoxide (DMSO) from 1 to $8 \%(\mathrm{v} / \mathrm{v})$ reduced sialylation but at the same time negatively impacted cell proliferation ${ }^{139}$. Nucleotide-sugar precursors modulate intracellular nucleotide-sugar pools and the resulting sialylation and antennarity levels. CHO-K1 cells secreting EPO incubated with $10 \mathrm{mM}$ glucosamine decreased sialylation on tetrasialylated glycans by $41 \%$, and the proportion of tetraantennary glycans by $37 \%$. On the other hand, with exceedingly high ammonia levels they decreased tetrasialylated glycans by $73 \%$, and the proportion of tetraantennary glycans by $57 \%^{141}$. Nitro benzene and fluoro benzoic acids were recognized as potential inhibitors of human sialidase including the most promising compound equipped with a $\mathrm{N}$-amide linked bulky biphenyl group. Nonetheless, further efforts are required to overcome conformational restrictions that result in weak inhibitory activities ${ }^{142}$. Following the example of fucosyltransferase inhibition, an azidosugar nucleotide derivative was identified as an efficient inhibitor by high-throughput quantitative MALDI-TOFMS-based screening against $\alpha 2,3$-SiaT (SiaT3Gal III) (IC $50=8.2 \mu \mathrm{M}$ ), but at the same time was a good donor substrate for $\alpha 2,6$-SiaT (SiaT6Gal I) $\left(\mathrm{K}_{m}=125 \mu \mathrm{M}\right){ }^{115}$. A recent article described reduced sialylation of a Fc-fusion protein in the presence of 0 to $20 \mathrm{mM}$ lithium chloride ( $\mathrm{LiCl}$ ) in the $\mathrm{CHO}$ culture medium $^{143}$.

\subsubsection{Glycation}

The presence of hexoses in the culture medium including the frequently used glucose and galactose may lead to glycation. A linear correlation was observed between the sugar concentration and the level of glycation in the tested range between 11.5 and $31 \mathrm{~g} / \mathrm{L}$ of sugar. Furthermore, glucose-only cultures exhibited about $0.3 \%$ glycation increase for each glucose $\mathrm{g} / \mathrm{L}$ addition, in contrast to a glycation increase in galactose-containing cultures by about 0.6 to $0.9 \%{ }^{75}$. Diabetes testing in both healthy subjects and patients revealed an association of trace element plasma concentrations $(\mathrm{Cu}, \mathrm{Zn}, \mathrm{Mg}$ ) with glycated hemoglobin levels, which increased at higher levels of $\mathrm{Cu}(16.4-21.5 \mu \mathrm{M})$ including $\mathrm{Cu} / \mathrm{Zn}$ ratio (1.08-2.03) and decreased levels of $\mathrm{Zn}(10.2-16.3 \mu \mathrm{M})$ and $\mathrm{Mg}(0.62-0.93 \mathrm{mM})^{144}$. In addition, it has been described that $\mathrm{Cu}^{2+}$ and $\mathrm{Fe}^{3+}$ play major roles in the generation of glycation products ${ }^{73}$. Another study recognized the excellent inhibitory effect of $\mathrm{Mn}^{2+}(5-20 \mu \mathrm{M})$ and the stimulating effect of $\mathrm{Zn}^{2+}(5-20 \mu \mathrm{M})$ on advanced glycation end products formation (AGE) ${ }^{74}$. In an animal study, rats accumulated AGE in various tissues due to fructose intake ${ }^{145}$. It was shown that betain inhibits glycation in vivo since it counteracted the elevation of reactive intermediate methylglyoxal and AGE levels in fructose-fed rat heart ${ }^{146}$. In a review of AGE inhibitors by foodstuffs, a great number of substances was identified including carnosine (a dipeptide: $\beta$-alanyl-L-histidine), curcumin (a diarylheptanoid), flavonoids, phenolic acids, and vitamins ${ }^{147}$. Hence, it is worthwhile to evaluate the applicability of these inhibitors in the frame of recombinant protein production from both a scientific and economic standpoint. 


\subsection{Charge Variants}

\subsubsection{Deamidation \& Isomerization}

Deamidation of asparagine residues, and often followed by aspartate isomerization, are major sources of instability and micro heterogeneity, and among other factors, might be induced by the cell culture medium composition ${ }^{148}$. Asparagine residues release ammonium, yielding an unstable succinimide intermediate in a spontaneous nonenzymatic reaction, and subsequently hydrolyze rapidly, in a ratio of about $3: 1$, into isoaspartate (iso-Asp) and aspartate (Asp) ${ }^{149,150}$. Asparagine (Asn) residues with glycine (Gly) on their C-terminus are the most prone to deamidation ${ }^{149}$. Both negative and absence of impact of deamidation on potency and immunogenicity have been reported on therapeutic proteins, whereas the influence on pharmacokinetics remains to be elucidated ${ }^{68}$. Glutamine (Gln) deamidation may also appear at an exceedingly slower rate than for Asn, and it is reported to be not of concern for biopharmaceuticals ${ }^{68}$.

Due to the chemical nature of the Asn deamidation and Asp isomerization, the degradation reaction is catalyzed by the hydroxide ions $\left(\mathrm{OH}^{-}\right)$and hence depends on the $\mathrm{pH}$ of the cell culture medium. Deamidation rates significantly increase with higher $\mathrm{pH}$, and accordingly higher $\mathrm{OH}^{-}$concentration ${ }^{151}$. Transposing the approach used in formulation development to design buffer compositions for protein storage at the most stable conditions is rather difficult, considering the host cell's requirement of culture media specifically adapted to its needs. Poloxamer 407 (Pluronic F127) at concentrations > 17\% (w/w) hampered the rate of deamidation up to $40 \%$ in an aqueous buffer at $35{ }^{\circ} \mathrm{C}^{152}$. While in addition to sucrose and poloxamer 407, trehalose, mannitol, glycerol, Tweens, buffer salts (histidine salts, phosphate salts), and ionic strength modifiers (sodium chloride) are frequently used in drug substance formulations ${ }^{153}$.

\subsubsection{Oxidation}

Cysteine (Cys), methionine (Met), tryptophan (Trp), histidine (His), and tyrosine (Tyr) residues are prone to oxidation, in that order ${ }^{154}$, especially when among other species the strong oxidant $\mathrm{OH}^{\cdot}$ radicals are formed in oxidative stress ${ }^{155}$. Free Cys in proteins are rare because of the considerably higher reactivity of the thiol group in Cys compared to other functional groups ${ }^{154}$. Most Cys are involved in disulfide bridges. Met oxidation has been reported to adversely affect both $\mathrm{mAb}$ structure and stability ${ }^{39}$. Various impacts of oxidation on biological activity, stability, and half-life of the therapeutic protein have been identified, and oxidationinduced aggregation may provoke immunogenicity reactions ${ }^{68}$. A recent study reported the use of oxidation reagents including hydrogen peroxide and tert-butyl hydroperoxide in combination with free tryptophan resulted in selective oxidation of methionine of IgG1 formulated at $25 \mathrm{mg} / \mathrm{mL}$ in $51 \mathrm{mM}$ sodium phosphate, $6 \%$ trehalose, and $0.04 \%$ polysorbate $20(\mathrm{pH} 6.2)^{156}$. 
The same researchers also showed how 2,2'-azobis(2-amidinopropane) dihydrochloride and free methionine jointly caused selective tryptophan oxidation, while not altering methionine oxidation.

The presence of transition metal ions, such as iron and copper $\left(\mathrm{Fe}^{2+}, \mathrm{Fe}^{3+}\right.$ and $\left.\mathrm{Cu}^{2+}\right)$, favor oxidation of amino acid residues in proteins. Oxidation was significantly reduced or nonexistent in oxidative conditions in an experiment with $1 \mathrm{mM}$ hydrogen peroxide $\left(\mathrm{H}_{2} \mathrm{O}_{2}\right)$ in the absence of iron and copper ${ }^{154}$. Oxidation can be effectively inhibited by the appropriate addition of antioxidants or free-radical scavengers, as shown during lyophilization of hemoglobin, where certain sugars circumvented oxidation ${ }^{157}$. Mannitol $(15 \%, \mathrm{w} / \mathrm{v})$, and sucrose $(6 \%, \mathrm{w} / \mathrm{v})$ in a slightly less effective way, protected the residues from oxidative stress even in the presence of metal ions ${ }^{154}$. In addition to sugars including glucose, chelating agents remove catalyzing metals and hence inhibit oxidation. Moreover, polyols at high concentrations displayed a protective effect on the oxidation of human relaxin and oxidation-labile peptides ${ }^{157}$. Likewise, water-soluble vitamin E (Trolox) and vitamin B6 (pyridoxine) had similar properties, due to their free-radical-scavenging capacities ${ }^{154}$.

\subsubsection{C- and N-Terminal Modifications}

C-terminal lysine (Lys) and N-terminal glutamine (Gln) charge variants are common for monoclonal antibodies ${ }^{41,53,61,158}$. Even though the mechanism of C-terminal Lys processing has yet to be fully understood, it was suggested that carboxypeptidases cleave the C-terminal Lys residues in a post-translational modification in cultured cells, yielding three antibody species of either 0 , 1, or 2 C-terminal Lys residues ${ }^{41}$ and lower isoelectric point. On the other hand, $\mathrm{N}$-terminal Gln cyclization to pyroglutamate results in more acidic antibodies with lower molecular weight following the removal of $\mathrm{NH}_{3}{ }^{159}$. There is no evidence that both C-terminal Lys and N-terminal Gln processing impact the biological activity of the molecule ${ }^{159,160}$ or affect immunogenicity or safety ${ }^{159}$. Furthermore, C-terminal proline (Pro) amidation of monoclonal antibodies has been described ${ }^{44,161,162}$. In a recent study the increase of C-terminal Pro amidation and hence basic variants was linked to the copper concentration in CHO fed-batch cultures $^{163}$. A copper concentration increase from 0.4 to $1 \mu \mathrm{M}$ was previously reported by the same company to augment basic variants from 6 to $15 \%$ at the end of the culture ${ }^{162}$. Minimal or negligible impact of C-terminal Pro amidation on potency and pharmacokinetic properties was demonstrated ${ }^{44}$. Even though $\mathrm{C}$ - and N-terminal modifications have not been reported to adversely affect safety ${ }^{41,68}$, potential correlations between glycosylation pattern and terminal modifications have been detected ${ }^{66}$, and hence highlight the importance to control the level of charge variants.

A study concluded that copper concentration in the cell culture medium was the most significant parameter affecting the C-terminal Lys variants of a monoclonal antibody rather than zinc according to previous publications ${ }^{53}$. Further investigations revealed the important role of copper/zinc ratio in both intracellular and extracellular C-terminal Lys processing, with 
high copper and low zinc levels favoring basic variants and as such C-terminal Lys ${ }^{53}$. However, large amounts of copper $(1 \mu \mathrm{M})$ in the culture medium might be undesirable because it can increase Pro amidation ${ }^{162}$. In another study, B-vitamins and iron significantly affected the drug substance color, and a correlation with increased acidic variants became evident, particularly when iron levels were responsible for the accentuated color. Potential mechanisms included enzymatic pyridoxine binding to Lys residues and free radical mediated oxidation of specific residues ${ }^{164}$. In $\mathrm{CHO}$ batch cultures yielding a chimeric anti-CD20 mAb lysine variant levels were strongly correlated with arginine (Arg) and Lys concentrations in the media. They increased from 18.7 to $31.8 \%$ with increasing Arg and Lys concentrations from 2 to $10 \mathrm{mM}^{165}$.

\subsubsection{Arginine Modifications by Methylglyoxal}

Chumsae et al. described the apparition of a new acidic peak in cell culture due to the modification of mAb by methylglyoxal (MGO), a highly reactive metabolite that can be generated from glucose, lipids, or other metabolic pathways ${ }^{166}$. Chemical modification of the guanidine side chain of arginine moeities at various sites of both variable and conserved domains by MGO gives rise to two adducts-dihydroxyimidazolidine and hydroimidazolone - with a mass increase of $72 \mathrm{Da}$ and $54 \mathrm{Da}$, respectively. They observed that the cell culture parameters affect how strongly the amino acid residue is modified and that the majority of modifications take place at CFR region of the antibody due to high flexibility. Moreover, they highlighted in their paper the fact that MGO may also react with lysine at a much slower reaction rate, forming a less stable product than with arginine.

\subsubsection{Global Acidic Species Charge Variant Modulation}

Recent attemps to reduce acidic charge variants employed media supplementation of bioflavonoids in four $\mathrm{CHO}$ cell lines expressing antibodies (IgG1) and immunoglobulins in fed-batch cultures. In particular, epigallocatechin gallate (EGCG) and rutin demonstrated effective reduction. The researchers hypothesize a cumulative effect of various of reduced species that their analytical method (LC/MS) did not perceive. EGCG addition (0.2 g/L) brought forth a $4 \%$ decrease and the presence of $1 \mathrm{~g} / \mathrm{L}$ rutin $6 \%$ reduction of IgG1 acidic variants. In immunoglobulin cultures rutin supplementation induced the largest effect at $0.05 \mathrm{~g} / \mathrm{L}$, which was largely superior to the effect in IgG1 cultures: $-14.3 \% 167$.

\subsection{Aggregates}

Protein aggregation should be limited, due to its potential to trigger loss of efficacy and immunogenic reactions, thus compromising the patient's health ${ }^{168}$. Purification processes are in general capable to sufficiently reduce the aggregation level of the cell culture broth ${ }^{169}$. 
Nevertheless, commercial manufacturing has a great interest to restrain the generation of aggregated forms during the upstream process, in order to maximize product yields. During cell culture, aggregation may occur within the host cell following protein expression, due to important accumulation of protein ${ }^{170}$. It results in intracellular aggregation owing to either the interactions of unfolded protein molecules or to inefficient recognition of the nascent peptide chain by molecular chaperones responsible for proper folding. Also, once the protein is secreted into the cell culture media, it may aggregate as a result of adverse environmental conditions ${ }^{46}$.

Osmolality impacts aggregation, as shown by the addition of $\mathrm{NaCl}$. Addition of $40 \mathrm{mM}$ decreased aggregation from 86 to $62 \%$ compared to the control culture ${ }^{171}$. In a batch culture addition of $56 \mathrm{mM}$ of $\mathrm{NaCl}$ aggregation significantly decreased and consequently high osmolality, and eventually completely vanished when the $\mathrm{NaCl}$ concentration by $85 \mathrm{mM}^{172}$. On the other hand, regardless of the medium osmolality, few aggregates were formed until the midexponential phase of cell growth, while they abundantly began to arise in the late-exponential and stationary phase. The limitations of hyperosmotic media became evident when cells eventually stopped growing and, as a consequence, viable cell density considerably diminished at such harsh conditions ${ }^{172}$.

Various reducing and oxidizing reagents including glutathionine, cysteine and copper sulfate proved to reduce the formation of protein aggregates or increase their stability in CHO cell culture harvests ${ }^{54}$. Other compounds, such as DMSO (1-8\%, v/v) and glycerol (1-2\%, v/v), exhibited protein stabilization capabilities ${ }^{139,173}$. In contrast, the addition of copper into the cell culture medium could slightly increase aggregation, whereas a supplementation of the culture medium with cysteine known for to its mild reducing characteristics on disulfide bond bridges, resulted in a substantial decrease in aggregate content and a corresponding increase in single chain species ${ }^{54}$. Cystine, the oxidized form of cysteine, also reduced high-molecularweight forms (HMW), but in contrast to cysteine, induced a much lower single-chain content increase. Combining cystine concentration and culture temperature shift was even more effective in decreasing protein aggregation. Furthermore, it resulted in greater sialylation and higher harvest titer ${ }^{54}$. Iron plays a major role in inhibiting the formation of aggregate forms, as shown in long-term drug substance stability studies, where the abundance of high-molecularweight species was significantly lower at low iron concentrations (0-4 ppm) in the presence of a chelator ${ }^{174}$. Surfactant containing culture media were reported to stabilize the expressed protein and thus reduce aggregation ${ }^{175,176}$. Specifically, the addition of $0.01 \%(\mathrm{v} / \mathrm{v})$ Polysorbate 80 into chemically defined concentrated feed media reduced overall aggregation levels by 2.6-2.7\% of two different CHO cell lines. However, in this study, it was rather the enriched feed media including Polysorbate 80 than the surfactant itself, which was responsible for the decrease in aggregation, since the medium osmolality significantly increased as a consequence of more concentrated medium ${ }^{176}$. A current work described the inhibitory capacities of trehalose, an approved additive used in drug substance and drug product formulation. It was observed that the addition of $150-200 \mathrm{mM}$ trehalose to the medium of a CHO cell culture prevented the polymerization and aggregation reaction of the recombinant protein ${ }^{177}$. The 
same research group also discovered a correlation between the level of aggregation and the abundance of $\mathrm{N}$-glycosylation species, exhibiting a reduction of both galactose and fucose residues in dimers and large aggregates to $70-80 \%$ of the amount in monomers ${ }^{178}$.

\subsection{Low-Molecular-Weight Species}

Fragmentation, the formation of low-molecular-weight species, is a common degradation type and can be attributed to the disruption of a covalent peptide bond by coexistent spontaneous and enzymatic reactions ${ }^{68}$. Even though the protein backbone is extremely resistant to nonenzymatic hydrolysis under physiological conditions, certain sites may become prone to fragmentation as a function of the presence of specific side-chains residues, such as Asp, Gly, Ser, Thr, Cys or Asn, which may facilitate cleavage due to increased flexibility of the local structure, solvent conditions ( $\mathrm{pH}$, temperature) and the presence of metals or radicals ${ }^{179}$ Furthermore, clipping may be observed as a result of the activity of proteases released by cells into the cell culture supernatant during the protein production process ${ }^{180-182}$. Adverse effects of fragmentation are various and potentially include reduced biological activity, shorter half-life and immunogenicity reactions and hence provoke patient safety issues ${ }^{68}$.

The pivotal part of copper in the non-enzymatic cleavage of proteins becomes apparent by varying its concentration. The reaction accelerates at increased concentration of cupric ions in solution, while on the contrary, the introduction of a chelating agent, such as EDTA, inhibits the fragmentation process. In phosphate-buffered saline solution at $37^{\circ} \mathrm{C}$ specific hinge cleavage by $\mathrm{Cu}^{2+}$ is significantly higher with respect to other di- and trivalent metal ions including $\mathrm{Mg}^{2+}$, $\mathrm{Mn}^{2+}, \mathrm{Zn}^{2+}, \mathrm{Fe}^{3+}$, and $\mathrm{Ni}^{2+179}$. In addition to copper, the hydrolysis of the hinge region of an IgG monoclonal antibody was found to be dependent on both iron (50 ppm) and histidine (2-10 mM) levels when changing the buffer system to histidine. However individually, iron and histidine have little or no effect on fragmentation at $37^{\circ} \mathrm{C}$. Iron-specific chelators can be used to inhibit cleavage in these conditions ${ }^{183}$. Nevertheless, it has been well described that in the presence of $\mathrm{Fe}^{2+}$, reactive oxygen species degrade biomolecules as they eventually form hydroxyl radicals $\left(\mathrm{OH}^{\prime}\right)$ in the so called Fenton reaction. These highly reactive species lead to fragmentation of proteins ${ }^{184}$.

Counter to chemical degradation, the thioredoxin (Trx) activity diminishes in the presence of 50-100 $\mu \mathrm{M}$ copper sulfate $\left(\mathrm{CuSO}_{4}\right)$, which consequently minimizes free thiols and in this way fragmentation ${ }^{185,186}$. Three enzyme systems including the Trx system-consisting of Trx, thioredoxin reductase (TrxR) and NADPH-, glucose-6-phosphate dehydrogenase (G6PD), and hexokinase have been identified to be responsible for antibody reduction and may be targeted to inhibit fragmentation. Few inhibitors have been identified for Trx such as divalent metal ions and disulfide compounds. On the other hand, gold complexes (ATG, ATM) are the most effective and selective inhibitors of TrxR. Dehydroepiandrosterone, epiandrosterone, pyridoxal 5'-phosphate, 1-fluoro-2,4-dinitrobenzene have effectively reduced G6PD activity 
and the addition of chelating agents (EDTA \& EGTA), citrate, distinct types of phosphates, 6-deoxy-6-fluoroglucose, 2-C-hydroxy-methylglucose, xylose, or lyxose inhibit hexokinase ${ }^{186}$. L-cystine exhibited chemical inhibitory capacities for a recombinant monoclonal antibody due to its function as competitive inhibitor for reducing enzymes ${ }^{55}$. A mixture of protease inhibitors including E64, leupeptin, benzamidine, E-amino caproic acid, pepstatin A, and EDTA was tested. It did not stop the peptide bond cleavage ${ }^{187}$. An exhaustive review of human protease inhibitors in the frame of protease-targeted drugs research provides a great number of small molecules either approved for clinical use or in development ${ }^{188}$. More recently, the status of the development of matrix metalloproteinase inhibitors presented selective inhibitors and highlighted also the challenges that had been encountered so far.

\subsection{Amino Acid Misincorporation}

As a result of using modern analytical technologies including intact mass measurement, peptide mapping, and tandem mass spectroscopy sequencing, the well documented misincorporation of amino acids occurring in proteins expressed in Escherichia coli was detected during recombinant protein production in mammalian hosts at high protein expression levels also ${ }^{189}$. Specifically, when cells are starved for Asn, the frequency of serine (Ser) incorporation at Asn positions increases during translation ${ }^{189}$. In spite of the fact that the underlying mechanisms have yet to be fully understood ${ }^{190}$, it is widely believed that the error rate during protein translation depends on factors such as the type of organism, the genotype and phenotype of the transfected cell lines, the codon usage, and the cell culture conditions ${ }^{191}$. When Asn abundance in the media is decreasing, Ser and Asn competition intensifies, leading to misacylation of tRNA ${ }^{A s n}$ by Ser. Serine substitution can be prevented by supplementing the culture medium with Asn ${ }^{189,190}$ and regularly feeding Asn $(6 \mathrm{~g} / \mathrm{L})$ throughout the culture to circumvent depletion of the latter ${ }^{190}$. Nonetheless, even at low levels of Asn in the medium, Ser misincorporation can be prevented through controlled feeding ${ }^{190}$, which may be a useful strategy to avoid important ammonia production as Asn synthetase is able to convert Asn to Gln, and eventually liberate ammonia to replenish the tricarboxylic acid cycle.

Recently, misincorporations of further amino acids were described, highlighting in particular the process-dependency of some of these types. It was shown that, in addition to the above mentioned Asn by Ser misincorporation, the levels of Asn by Lys, Ser by Asn, and Ser by Arg substitutions varied significantly between two distinct process conditions ${ }^{191}$. Codon mismatches seem to be the principal reason of amino acid misincorporation for recombinant proteins under balanced nutrient conditions, and since on top of the cell line the environment affects misincorporation, media and feed optimization is essential. 


\subsection{Components Affecting Multiple Quality Attributes}

Table 2.2 gives an overview of the in the previous section presented culture media components that are reported to affect multiple quality attributes simultaneously.

\subsection{Outlook}

Like the quote of the great musician Johann Sebastian Bach, "It's easy to play any musical instrument: all you have to do is touch the right key at the right time and the instrument will play itself" 192 , in cell culture media development the challenge is to add the right components at the right concentration to tailor the quality attributes of recombinant proteins, and in particular of new biological entities such as biosimilars and biobetters. The intensive research efforts that have been performed, aimed to elucidate the mechanisms underlying the various post-translational modifications shaping the therapeutic protein. Published data demonstrate the feasibility and the great potential of quality engineering through media design. Rather than modifying the gene expression of the cell line, including knockout techniques or changing the host cell type to obtain the desired features of the recombinant protein, media design is an attractive alternative to significantly modulate the protein function. Media optimization is therefore expected to become an essential part to enhance the pharmacological properties. Despite the plethora of results and findings, more specific work in mammalian cell culture suitable conditions is required, and the applicability of the proposed strategies have to be further evaluated for manufacturing up-scaling during clinical trial phases and eventual commercial manufacturing at industrial scales. Still nowadays, it is a great challenge to cope with the enormous complexity and multiple pathways in the host cell, because we are lacking thorough understanding of the relationships between the processes taking place within the cells, specifically the interactions between the media components, which is pivotal to consistently offer to patients more efficient and safer treatments. Thus, it is of great value to streamline cell culture media development by creating a library of suitable supplements that can modulate specific protein quality attributes, by elucidating how these supplements affect the underlying pathways in the host cell, and finally, by bringing efficient media optimization strategies into action, in a manner that, in the future, we touch the right key. 


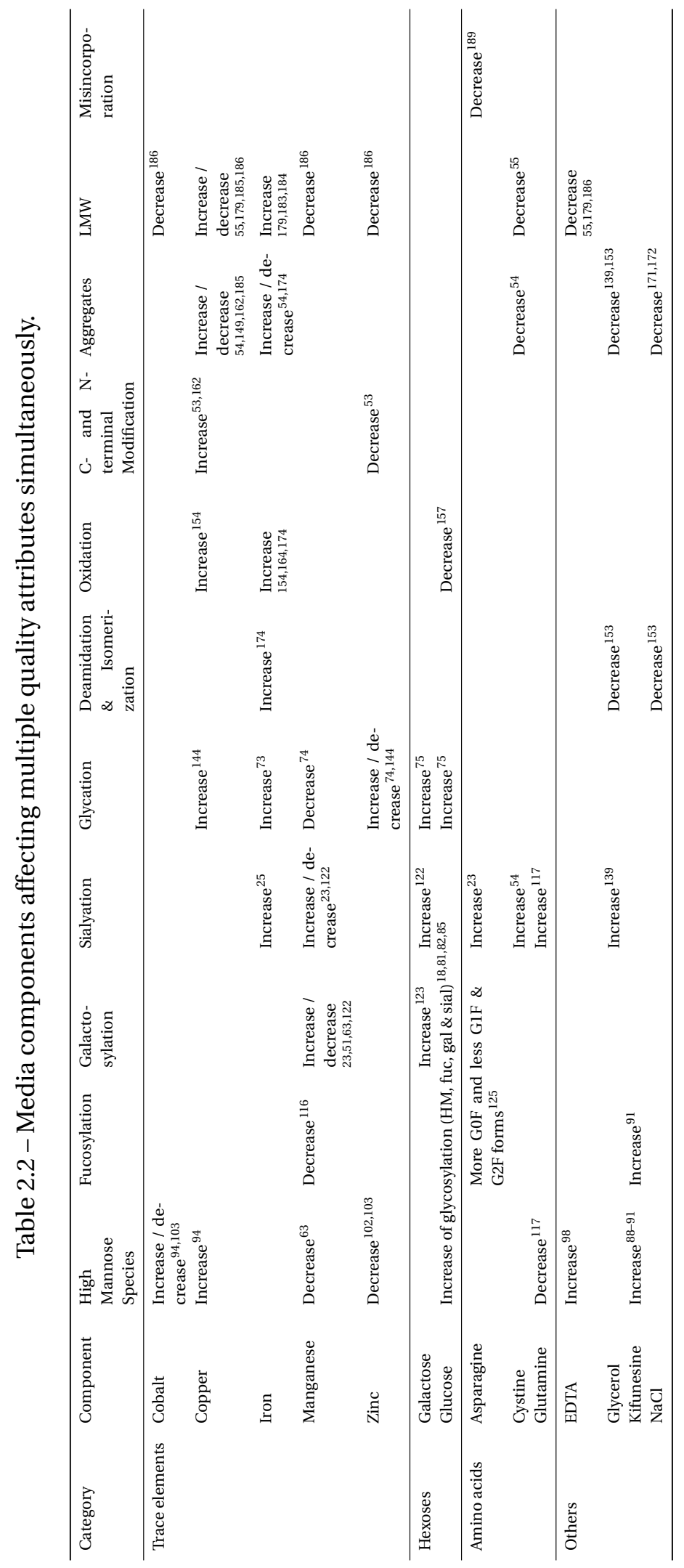


Part II

Research 



\section{Chapter 3}

\section{Research Objectives}

The aim of this thesis was to lever cell culture media design for recombinant protein quality modulation according to the previous part that highlighted its great potential for tuning of the quality profile of recombinant proteins. The research project was divided into four distinct topics to reach this goal. In fed-batch cultures, the potential of medium and feed supplementation was evaluated with compounds (commonly known media components and novel supplements), which enhance or inhibit post-translational modifications including glycosylation and the generation of low-molecular-weight species. The next step consisted in the development of a rational experimental design method to identify the best glycosylation modulating compounds among many media supplements and to spot potential synergistic effects. Subsequently, metabolomic profiling and multivariate modelling provided further mechanistic understanding that laid a foundation for N-glycosylation control in routine manufacturing. Finally, the developed library of glycosylation modulating compounds was used to assess the effect of the induced glycosylation changes on the biological activity. The outline of each specific part is provided hereafter.

\section{To Create a Quality Modulation Compound Library}

Chapter 4 presents how cell culture media supplemented with raffinose reproducibly increased the level of high mannose glycans in various cell culture systems, including 96-deepwell plates, shake tubes and 3.5-L bioreactors. Both specific and non-specific inhibitors of galactosyltransferase consistently and reproducibly reduced the abundance of terminal galactose when added into the medium or the bolus feed as described in chapter 5 . While the main focus of this research part consisted of glycan modulation, chapter 6 describes exploratory tests in high-throughput screening experiments intended to identify causal relations between the level of medium components and the degree of low-molecular-weight species in the supernatant. Subsequent tests at greater volume served to reproduce the outcomes and to further investigate medium supplements that potentially favor disulfide bond reduction. 


\section{To Develop a High-Throughput Screening Experimental Strategy}

With the objective to reduce complexity and to streamline the process-development workflow, chapter 7 depicts the development of a parallel experimental design method for efficient screening of cell culture media supplements to improve the product quality. Seventeen compounds were separated into five different groups of parallel design-of-experiment of $\mathrm{CHO}$ fedbatch cultures in 96-deepwell plates to minimize both dilution effects and the repercussions due to non-optimal conditions. Multivariate analysis was used to select the best performing glycosylation modulators. The final part of this chapter was aimed at confirming the outcome of the selection process, using D-optimal quadratic design in shake tubes. Its purpose was also to provide a solid basis for sequential process development at larger scales. Moreover, the developed experimental strategy was used in 96-deepwell plates for the identification of compounds affecting the charge profile, the level of aggregation and low-molecular-weight species.

\section{To Perform Metabolomic Profiling and Multivariate Modelling}

With the tight control of product quality in routine manufacturing in mind, intracellular and extracellular non-targeted metabolomic profiling of four different 3.5-L bioreactor process formats, using the same cell line provided insight in the distinct metabolite profiles. The part described in chapter 8 intended to pinpoint metabolites featuring similar patterns as the distinct extracellular lactate profiles. The bioreactor scales also enabled to study the timely evolution of the glycan pattern throughout the culture. Intracellular nucleotide sugar levels provided an indication about the importance of the substrate level with respect to enzyme activity or gene expression. Finally, by means of multivariate analysis the analysis of the huge data set was simplified. Calling on partial-least-square (PLS) modelling, the goal was to calibrate a PLS observation model with extracellular metabolite levels to predict the glycosylation pattern at a specific time point based upon the extracellular measurement at that very moment.

\section{To Assess the Effect of Glycosylation Modulation on the Biological Activity}

In addition to the built glycosylation modulation compound library, enzymatic glycoengineering technology was used as an alternative approach to generate a wide range of glycovariants. Chapter 9 describes the strategy applicable both to a monoclonal antibody and to an antibody fusion molecule, which is needed for the bioactivity assessment of the glycan differences between the biosimilar and the reference medicinal product (RMP), the health agencies may ask for in the drug registration process. The combination of cell culture medium supplementation and enzymatic glycoengineering produced great differences of the distinct glycosylation patterns, and as a result biological activity assays were able to pick up the effects of the induced glycosylation changes. The use of glycopeptide mass spectrometry technology gave insight in each local glycosylation pattern of the three glycosylation sites of the antibody fusion molecule. This technique also provided information about the degree of antennarity of each glycosylation site. 


\section{Chapter 4}

\section{Cell Culture Media Supplemented with Raffinose Reproducibly Enhances High Mannose Glycan Formation ${ }^{1}$}

\subsection{Introduction}

A significant amount of research has been performed to produce monoclonal antibodies with increased effector functions including antibody-dependent cell-mediated cytotoxicity $(\mathrm{ADCC})^{91}$ and cell-dependent cytotoxicity (CDC) ${ }^{200}$. It has been shown that the N-linked glycan affected the Fc $\gamma$ IIIIa receptor binding and ADCC activity of the antibodies ${ }^{201}$. Like afucosylated glycans, high-mannose species induced increased ADCC, thus reflecting their Fc $\gamma$ IIIIa-binding affinity, nonetheless to a lesser extent than afucosylated complex glycans ${ }^{197}$. Recently, many companies have increased the number of biosimilars in development due to patent expiry of biologics ${ }^{10}$. In that context, the identification of levers affecting recombinant protein quality has become a main focus. Glycoheterogeneity occurs naturally in the Golgi apparatus $^{202}$. Gene and expression levels, as well as spatial localization of the enzymes and nucleotide-sugar substrate influence the level of antennarity and the degree of glycan transformation ${ }^{18}$. In biosimilar development, the aim is the consistent expression of a highly similar glycan fingerprint compared with the originator molecule ${ }^{6}$.

Researchers reported that in supplemented medium the cell transformed fluorinated peracetylated fucose and sialic acid into the corresponding fluorinated nucleotide sugars by means of its salvage pathway ${ }^{112}$. They observed a specific and efficient inhibition of the fucosyl and sialyl transferases, respectively, when adding one of these compounds to the media at

1. Submitted, D. Brühlmann, A. Muhr, R. Parker, T. Vuillemin, B. Bucsella, F. Kalman, S. Torre, F. La Neve, A. Lembo, T. Haas, M. Sauer, J. Souquet, H. Broly, J. Hemberger and M. Jordan, Cell Culture Media Supplemented with Raffinose Reproducibly Enhances High Mannose Glycan Formation. 
the micromolar level. Instead of inhibiting one of the glycosylation transformation enzymes in the endoplasmic reticulum (ER) or the Golgi apparatus, the substrate generation may be targeted. Cultures supplemented with $\mathrm{N}$-acetylglucosamine (GlcNAc) at the millimolar level resulted in reduced complexity of glycan profiles, hence favoring the G0 glycoform ${ }^{204}$. Media supplementation with kifunensine, a potent $\alpha$-mannosidase I inhibitor entailed an increase of oligomannose containing monoclonal antibodies $(\mathrm{mAb})^{91}$. That study exhibited increased ADCC activity due to the increase of high mannose (HM) glycans; in particular mannose 8 and 9 entities. Supplementation of sucrose and tagatose into fed-batch media effectively redistributed the $\mathrm{N}$-glycan glycoform profile toward HM species ${ }^{108}$. In a perfusion process, a novel approach utilized mannose as a carbon source and the ratio of mannose to the total hexose in the feed media correlated with the abundance of HM glycan species ${ }^{109}$.

In this study, a robust approach is presented to increase the abundance of high mannose type monoclonal antibodies. The metabolic engineering approach consisted of supplementing cell culture media with raffinose, a naturally occurring trisaccharide composed of galactose, glucose, and fructose. Supplementation was assessed in high-throughput systems including 96-deepwell plates and shake tubes. 96-deepwell plates have proven to be a reliable system to screen raw materials impacting product quality attributes of recombinant proteins ${ }^{25,27,28}$. Confirmation of the findings was conducted in lab-scale 3.5-liter bioreactors. Although HM should be minimized due to immunogenic reactions ${ }^{205}$, a method was developed, which may be utilized in the frame of biosimilar development to match the quality profile of the reference medicinal product (RMP). Raffinose, a water soluble carbohydrate, was first found in the Australian Eucalyptus manna, in cotton seed, in sugar beet molasses and both barley and wheat ${ }^{206}$. Nowadays, it is known that raffinose can be found in all plants ${ }^{207}$. Raffinose family oligosaccharides have miscellaneous functions in plants including transport and storage of carbon and are involved in the protection against abiotic stress in plants ${ }^{208}$. Several studies described the effect of raffinose on various metabolic pathways of other cell types. Prebiotic treatment of fertile eggs injecting raffinose into the amniotic fluid significantly increased the relative expression of aminopeptidase, sucrase isomaltase, ATPase, and sodium glucose co-transporter 1 . As a result, the iron bioavailability was altered ${ }^{209}$. In humans, raffinose intake was correlated with leukotoxic effects and oxidative stress ${ }^{210}$. The results presented in this work demonstrate that in fed-batch processes raffinose supplementation reproducibly increases the amount of HM glycans.

\subsection{Materials and Methods}

\subsubsection{Inoculum Preparation}

Two recombinant cell lines were used in the frame of this study. A CHO-K1 derived clonal cell line expressing a humanized monoclonal IgG1 antibody (cell line 1) and a CHO-S derived clonal cell line expressing a human monoclonal IgG1 antibody (cell line 2). Cells were first 
expanded in multiple passages in shake tubes or shake bottles in Merck proprietary medium containing methionine sulfoximine (MSX) for at least 14 days in a shaker incubator at $36.5^{\circ} \mathrm{C}$, $5 \% \mathrm{CO}_{2}, 80 \%$ humidity and $320 \mathrm{rpm}$ agitation (ISF1-X, Adolf Kühner, Birsfelden, Switzerland or Multitron Cell, Infors HT, Bottmingen, Switzerland).

\subsubsection{Cell Culture in 96-Deepwell Plates}

The fed-batch cell culture was performed on a robotic liquid handling platform (Biomek FX, Beckman Coulter, Brea, CA). CHO-K1 cells were seeded into a shaking 96-DWP filled with Merck proprietary medium enriched with 0 to $50 \mathrm{mM}$ raffinose (Sigma, Darmstadt, Germany) in the absence of MSX at a viable cell density of $0.20 \times 10^{6}$ viable cells $/ \mathrm{mL}$ and CHO-S cells at $0.30 \times 10^{6}$ viable cells $/ \mathrm{mL}$. A second round of experiments was carried out, using a constant medium osmolality approach. The medium was enriched with 0 to $128 \mathrm{mM}$ raffinose, and subsequently, distinct amounts of $\mathrm{NaCl}$ added to reach a final osmolality of $315 \mathrm{mOsm} / \mathrm{kg}$ in all experimental conditions. The plates were incubated with vented lids to minimize evaporation in a shaker incubator at $36.5{ }^{\circ} \mathrm{C}, 5 \% \mathrm{CO}_{2}, 90 \%$ humidity and $320 \mathrm{rpm}$ agitation (ISF1-X, Adolf Kühner, Birsfelden, Switzerland) for 14 days. $400 \mathrm{~g} / \mathrm{L}$ glucose solution, chemically defined feed containing over 30 components and alkaline amino acid solution were added on day 3, 5, 7, 10 and 12. Prior to each feeding and at the end of the culture (day 14), samples ( $\leq 40 \mu \mathrm{L}$ ) were drawn for growth and viability assessment and product titer quantification.

\subsubsection{Cell Culture in Shake Tubes}

CHO-K1 cells were seeded into a TPP® TubeSpin bioreactor tubes (referred to shake tubes or $S T$ ) filled with either Merck proprietary medium enriched with 0 to $100 \mathrm{mM}$ raffinose adjusted with $\mathrm{NaCl}$ to $315 \mathrm{mOsm} / \mathrm{kg}$ in the absence of MSX, or with Cellvento CHO200 (Merck Life Science, Darmstadt, Germany) enriched with 0 to $50 \mathrm{mM}$ raffinose at a viable cell density of $0.20 \times 10^{6}$ viable cells $/ \mathrm{mL}$ and CHO-S cells at $0.30 \times 10^{6}$ viable cells $/ \mathrm{mL}$. The ST were incubated in a shaker incubator at $36.5{ }^{\circ} \mathrm{C}, 5 \% \mathrm{CO}_{2}, 80 \%$ humidity and $320 \mathrm{rpm}$ agitation (ISF1-X, Adolf Kühner, Birsfelden, Switzerland) for 14 days. Into the ST filled with Merck proprietary medium, chemically defined feed (CDF) containing over 30 components and an alkaline amino acid solution were added on days 3, 5, 7 and 10, while the $400 \mathrm{~g} / \mathrm{L}$ glucose solution (GlcS) was added on these days and day 12 as well. The Cellvento CHO200 containing tubes were fed with Cellvento Feed-200 (Merck Life Science, Darmstadt, Germany) on days 3, 5, 7 and 9 and with cysteine/tyrosine stock solution on the same days according to supplier recommendations. The $400 \mathrm{~g} / \mathrm{L}$ GlcS was added on days 3, 5, 7, 9 and 12. Prior to each feeding and at harvest (day $14)$, aliquots $(\leq 2.5 \mathrm{~mL}$ ) were taken for viable cell counting, extracellular metabolite profiling (not shown) and product titer determination (not shown). 


\subsubsection{Cell Culture in 3.5-L Bioreactors}

The passaged CHO-K1 cells were seeded in 3.5-L bioreactors, (Biostat B, Sartorius, Göttingen, Germany; final volume: $3.0 \mathrm{~L}$ ) filled with Merck proprietary medium enriched with 0 to $30 \mathrm{mM}$ raffinose in the absence of MSX at $0.20 \times 10^{6}$ viable cells $/ \mathrm{mL}$. The CDF and the alkaline amino acid solution were added on day 3, 5, 7 and 10. Glucose was fed daily from day 3 to the end of the culture. 10 to $15 \mathrm{~mL}$ samples were collected every day for growth and viability assessment, $\mathrm{pH}, \mathrm{pCO}_{2}, \mathrm{pO}_{2}$, extracellular metabolites, osmolality and titer quantification (not shown).

\subsubsection{Cell Counts, Cell Viability and mAb Titer Analysis}

Growth and viability assessment of 96-DWP was performed on a Guava easyCyte (Merck Lifesciences, Darmstadt, Germany). The mAb titer was analyzed on day 14 on an Octet ${ }^{\circledR}$ (forteBIO, Menlo Park, CA) using Protein A sensors. Each sample was diluted 20 to 40 times into a dilution buffer (PBS pH 7.4, BSA $1 \mathrm{~g} / \mathrm{L}$, Tween 20 at $0.02 \%$ ). The sensors were regenerated with a buffer containing $10 \mathrm{mM}$ glycine- $\mathrm{HCl}$ at $\mathrm{pH} 1.5$ and neutralized with the dilution buffer.

\subsubsection{Glycan Analysis}

At the end of each 96-DWP, ST and 3.5-L bioreactor fed-batch, the supernatant was purified on small-scale affinity columns (PhytipsVR, PhyNexus, San Jose, CA). The N-glycosylation profile of the 96-DWP eluates were analyzed by capillary gel electrophoresis with laser-induced fluorescence detection (CGE-LIF, DNA genetic analyzer 3130XL, Life Technologies, Darmstadt, Germany) $^{211}$. The neutralized ST and 3.5-L bioreactor samples were denatured by IAA in $0.6 \mathrm{M}$ denaturation reagent (GlykoPrep-plus, Europa Bioproducts, Cambridge, UK) and reduced. Following purification, the samples were labelled with 2-amino-benzamide and then dried for 3 days. The dried samples were dissolved in $50 \%$ ACN and subsequently injected into the $100 \mathrm{~mm}$ UPLC column in length supplied by Waters Corporation, Milford, MA, USA and eluted, using a gradient.

\subsubsection{Intracellular Nucleotide and Nucleotide Sugar Profiling}

ST were inoculated with cell line 1 at $0.35 \times 10^{6}$ viable cells in medium enriched with 0 to $100 \mathrm{mM} .3 \times 10^{7}$ viable cells were collected on day 3 (1 part) and immediately quenched in 4 parts of $\mathrm{NaCl} 0.9 \%(\mathrm{w} / \mathrm{v})$ at $0{ }^{\circ} \mathrm{C}$. Subsequent centrifugation at $1000 \mathrm{~g}$ for 1 minute at $0{ }^{\circ} \mathrm{C}$, the supernatant was removed and the cell pellet flash-frozen in liquid nitrogen. Subsequently, they

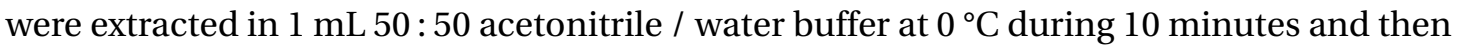
vortexed and centrifuged at $4000 \mathrm{~g}$ for 5 minutes at $0{ }^{\circ} \mathrm{C}$. The nucleotide and nucleotide sugar (NS) extracts were stored at $-80^{\circ} \mathrm{C}$. The sample preparation and analysis were performed by 
capillary zone electrophoresis method with direct detection at $260 \mathrm{~nm}$ in duplicates according to the previously described method ${ }^{212}$.

\subsubsection{Transcriptomics Analysis}

On day $5,10^{7} \mathrm{CHO}-\mathrm{K} 1$ cells were collected in ST culture enriched with 0 to $100 \mathrm{mM}$ raffinose, RNAprotect (Qiagen, Venlo, Netherlands) added according to supplier's instructions and then the total RNA was isolated from the cell pellet, using the RNeasy Mini Kit (Qiagen, Venlo, Netherlands) according to the supplier's and internal work instructions (not shown). RNA integrity was assessed using Agilent Bioanalyzer (Agilent Technologies, Santa Clara, CA) by means of RNA 6000 Nano kit (Agilent Technologies, Santa Clara, CA). RNA integrity numbers, calculated from ribosomal $18 \mathrm{~S}$ and $28 \mathrm{~S}$ RNA peak ratios, were required to be greater than 8 for subsequent library preparation. After verifying the integrity, $1 \mu \mathrm{g}$ of the total extracted RNA for each sample was used to prepare sequencing libraries by means of the Illumina TruSeq Stranded Total RNA Sample Preparation Kit following the manufacturer's protocol. The obtained libraries were finally evaluated by QUBIT® 2.0 Fluorometer (Invitrogen, Waltham, MA) and High Sensitivity DNA kit (Agilent Technologies, Santa Clara, CA) to check the quantity and size. Individual libraries were prepared using unique index adapters and pooled together. Mixed pools were loaded on the Illumina NextSeq 500/550 High-Output Flow cell v2 (300 cycles) and sequenced using the NextSeq500 instrument (Illumina, San Diego, CA) with $75 \mathrm{nt}$ paired-end reads for a total of 158 cycles. Data were collected, using NCS v1.4.1.2 and transferred automatically into the computing platform for the subsequent analysis.

Sequencing data in FASTQ format were produced using bcl2fastq v2.15.0.4 $4^{213}$. The fastq files, for each sample, were analyzed by Trimmomatic v0.27 214 , that cut the Illumina adapter sequences and filter the low quality reads where impossible to cut. After the first data processing, sequencing reads were mapped to the sequences and the transcriptome annotations of $\mathrm{CHO}$ genome $^{215}$ using tophat v2.0.13 tool ${ }^{216}$ and the embedded bowtie2 alignment tool ${ }^{217}$. For tophat standard options were used. Gene level raw counts of mapped reads were performed by HtSeq v0.6.1p1 ${ }^{218}$ with unstranded setting. Data results were managed with BioconductorDESeq package v1.14.0 ${ }^{219}$ in order to identify a differential expression between wild type and mix of biological and technical replicates of the treated samples.

\subsection{Results}

\subsubsection{Cultures in 96-Deepwell Plates}

The first test focused on the effect of a wide raffinose concentration range in the cell culture medium on the high mannose abundance of two cell lines. Figure 4.1 shows the fold glycosylation change in function of the raffinose concentration in the production medium prior to 
seeding with CHO-K1 cells. The increase of high mannose species (Man5, Man6 and Man7) correlated with the trisaccharide level in the medium of each culture. At low concentrations (0.1 to $5 \mathrm{mM}$ ), HM species exhibited an up to 1.1-fold increase, at $10 \mathrm{mM} 1.3$-fold, at $30 \mathrm{mM}$ they doubled, and at $50 \mathrm{mM}$ these glycoforms were 2.8 times more abundant than in the nonsupplemented control cell culture. The abundance of galactosylated species slightly decreased at concentrations ranging from 0.1 to $1 \mathrm{mM}$. At higher concentrations, galactosylation was favored and thus reached the peak at $50 \mathrm{mM}$ (1.2-fold). The presence of raffinose brought about lower levels of fucosylation. At $50 \mathrm{mM}$, the largest decrease was observed (-11\%). No sialylated species were detected at all tested concentrations. Raffinose yielded limited amounts of new glycan species: the change of non-identified species remained within a range of $1.00 \pm 0.08$. As shown in figure 4.2, the cell cultures exhibited comparable cell growth with respect to the control up to a raffinose concentration of $30 \mathrm{mM}$. At $50 \mathrm{mM}$, a decrease of the viable cell density was observed. At concentrations up to $10 \mathrm{mM}$, the cultures yielded comparable product titers at harvest (day 14). Beyond this concentration the product titers decreased. At a raffinose concentration of $30 \mathrm{mM}$, the harvested product amounted to $82 \%$, and respectively $50 \mathrm{mM}$, $76 \%$ of the control.

A

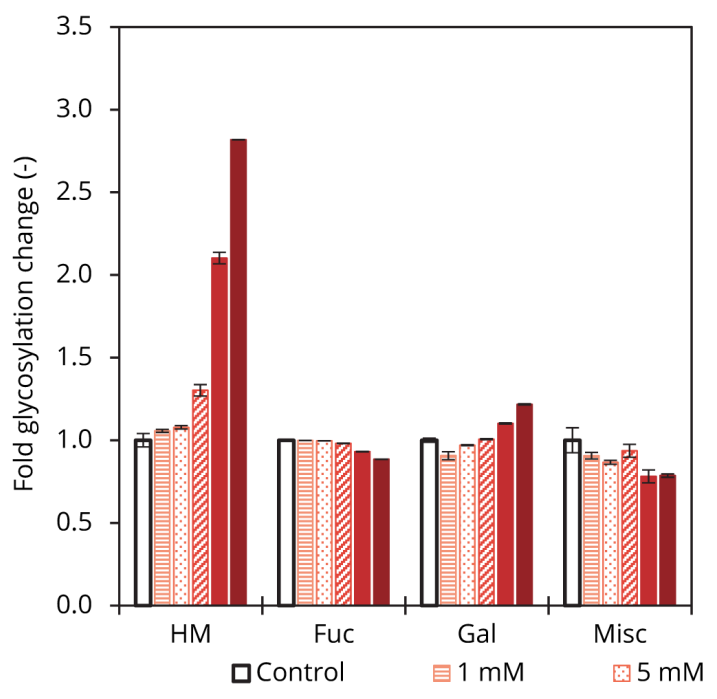

B

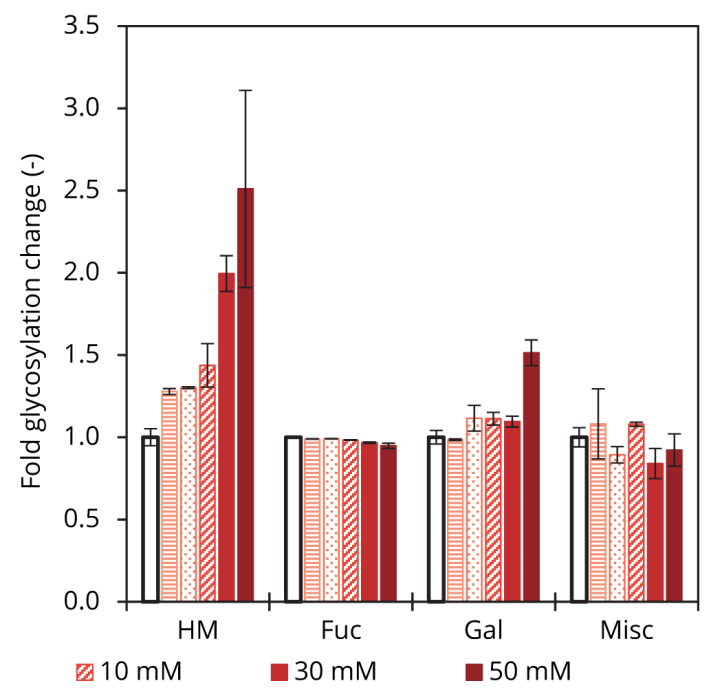

Figure 4.1 - Fold glycosylation change in function of raffinose concentration $(0-50 \mathrm{mM})$ in the production medium prior to 96-DWP inoculation with cell line 1 (A) and cell line 2 (B). The control cultures $(0 \mathrm{mM})$ were conducted in 4 replicates and raffinose supplemented conditions in duplicates. Error bars show variability within replicates. HM: high mannoses, Fuc: fucosylated species, Gal: galactosylated species, Misc: miscellaneous.

In the same way, cell line 2 cultures exhibited a proportional increase of high mannose glycans with increasing raffinose concentrations in the cell culture medium. $30 \mathrm{mM}$ raffinose doubled HM, while $50 \mathrm{mM}$ brought about 2.5 times more abundant HM (figure 4.1). Fucosylation slightly decreased and galactosylation increased with increasing supplement concentrations. At $50 \mathrm{mM}$, galactosylation was 1.5 times higher than in the control. The variation of the 
A: Cell line 1

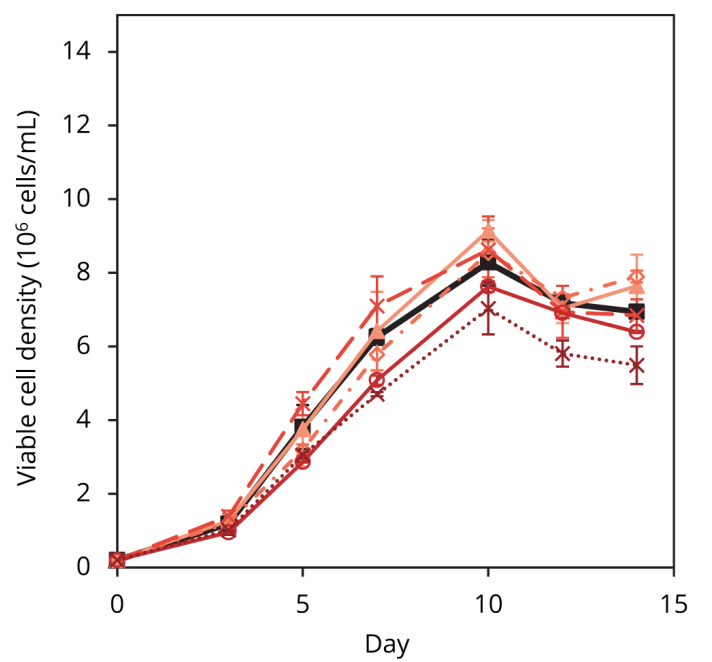

B: Cell line 2

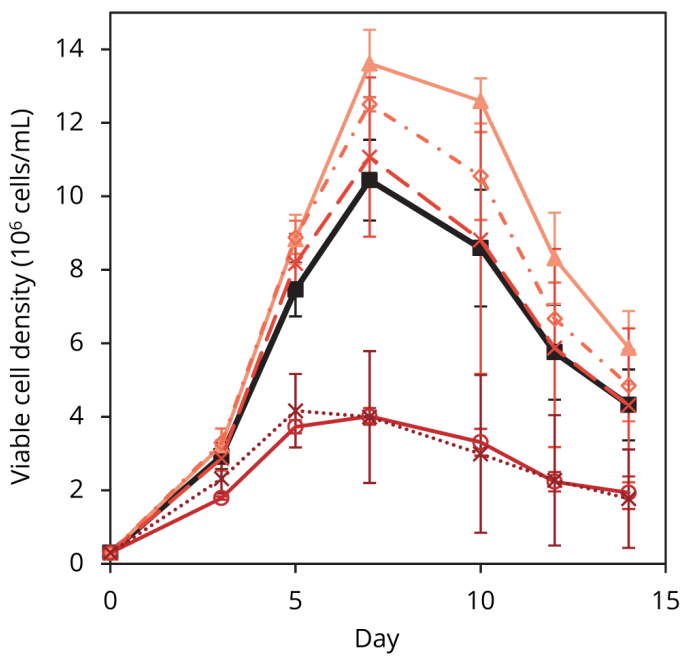

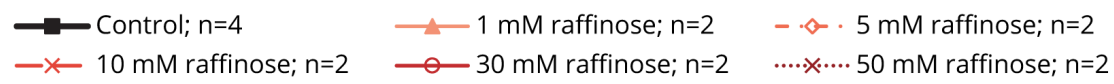

C: Cell line 1

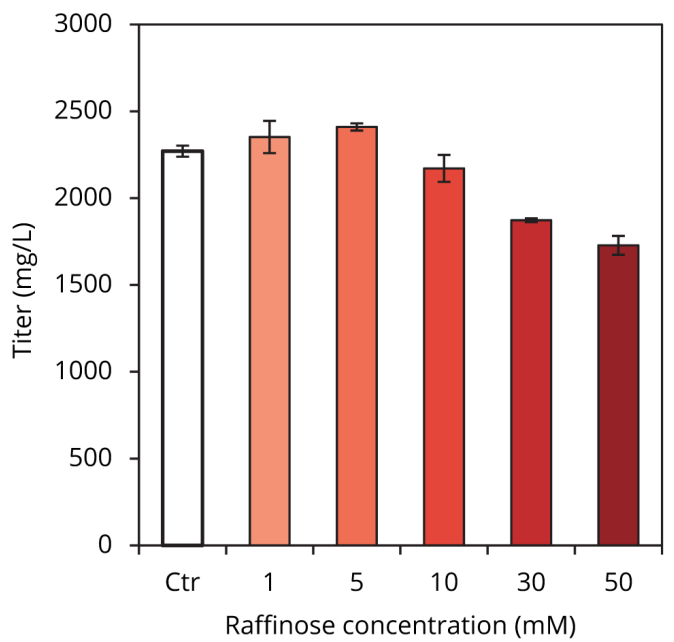

D: Cell line 2

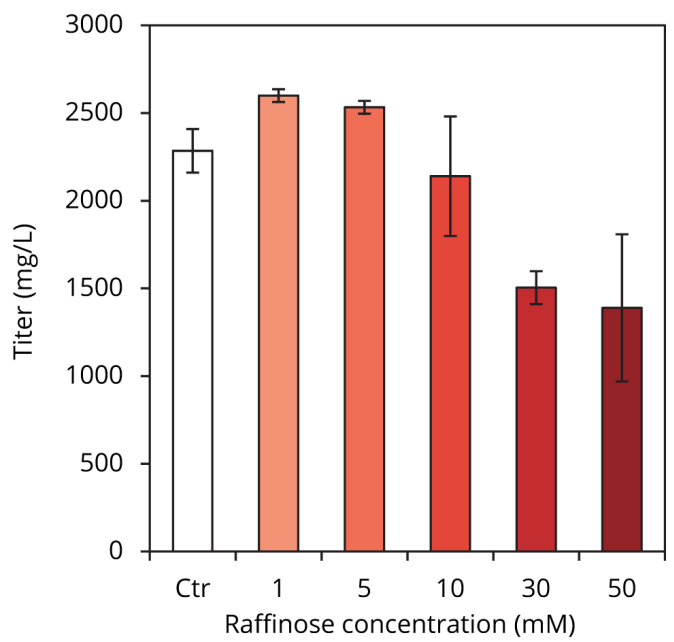

Figure 4.2 - (A) Viable cell densities of cell line 1 cultures supplemented with 0-50 mM of raffinose. (B) Viable cell densities of cell line 2 cultures supplemented with 0-50 mM of raffinose. (C) Harvest titers (day 14) of cell line 1 cultures. (D) Harvest titers (day 14) of cell line 2 cultures. All raffinose supplemented cultures were performed in duplicates and the control in four replicates in 96-DWP. 
miscellaneous peaks appears to be larger than in the cell line 1 cultures. While cell growth and titer at concentrations of up to $10 \mathrm{mM}$ raffinose were comparable to the control cultures, increasing supplementation ( 30 and $50 \mathrm{mM}$ ) strongly impeded growth. The latter reached peak cell densities of about $4 \times 10^{6}$ viable cells $/ \mathrm{mL}$ instead of 10 to $14 \times 10^{6}$ viable cells $/ \mathrm{mL}$. Product titers at harvest stalled at 60 to $65 \%$ of the control levels.

\subsubsection{Cultures at Constant Medium Osmolality}

At constant medium osmolality $(315 \mathrm{mOsm} / \mathrm{kg}$ ) growth inhibition effects of cell line 2 decreased (figure 4.3). At $30 \mathrm{mM}$ raffinose cell growth was still comparable to the control. At 50 and $65 \mathrm{mM}$ raffinose peak cell density reached 8.9 to $9.5 \times 10^{6}$ viable cells $/ \mathrm{mL}$. Raffinose concentrations $\geq 80 \mathrm{mM}$ strongly reduced cell growth. The cultures yielded comparable titers as high as a concentration of $65 \mathrm{mM}$ raffinose in the medium. Beyond, titers caved in. Constant osmolality proved to be a successful approach enabling to limit detrimental effects on both growth and titer of cell line 2 cultures. It was possible to further increase raffinose supplementation at constant medium osmolality with limited impact on growth up to $65 \mathrm{mM}$. Regardless of the constant osmolality of all raffinose supplemented conditions as well as the control, high raffinose concentrations impaired cell metabolism thus resulting in a massive growth reduction and lower product titers.

\subsubsection{Cultures in Shake Tubes and 3.5-L Bioreactors}

The constant osmolality approach was used to reproduce the findings in ST. This strategy made possible to test a wide raffinose concentration range (0 to $100 \mathrm{mM})$ in both cell line 1 and 2 cultures. The lower experimental throughput at this scale enabled to use 2AB-UPLC glycan analysis instead of CGE-LIF and thus enhanced the glycan resolution. The use of 2AB-UPLC avoids co-elution of HM with other glycan structures. Figure 4.4 shows proportional increase of HM with increasing raffinose presence in the medium for cell lines 1 and 2. Raffinose mainly favored Man5 structures. At $100 \mathrm{mM}$ of raffinose, cell line 1 expressed $6.7 \%$ Man5 and Man6 peaked at $0.24 \%$. While traces of Man8 were found $(0.07 \%)$, no Man4 and Man7 were detected. The raffinose supplementation of Cellvento CHO-200 medium also increased HM formation of cell line 1. Its abundance correlated with the trisaccharide concentration in the medium. Since the constant medium approach was prevented by the use of a commercial medium, concentrations between 0 and $50 \mathrm{mM}$ were tested. At $50 \mathrm{mM}$ raffinose, Man 5 reached $6.0 \%$ and Man6 $0.16 \%$. In both media, no Man4 nor Man7 were detected. While small Man8 levels were detected, increasing raffinose concentrations did not entail Man8 increases. A slightly different picture was obtained for cell line 2 in proprietary medium supplemented with raffinose while maintaining the osmolality constant. Man5 was the most abundant high mannose oligosaccharide (9.2\% at $100 \mathrm{mM}$ raffinose). $0.07 \%$ of Man $4,1 \%$ of Man6 and $0.8 \%$ Man7 were present and their levels grew with increasing raffinose concentration. Cell culture 
A

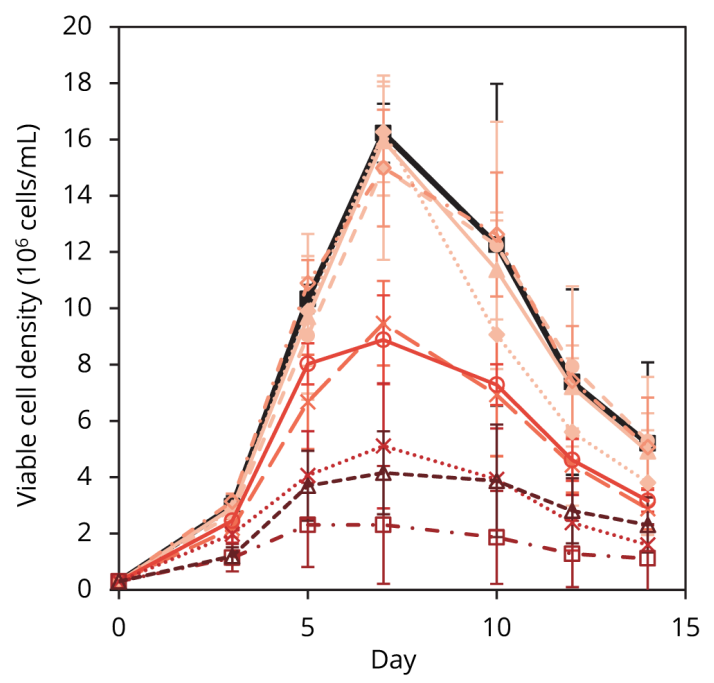

Control; $n=4$
10 mM raffinose; $n=6$
-65 mM raffinose; $n=4$

-- - -. 128 mM raffinose; $n=6$
B

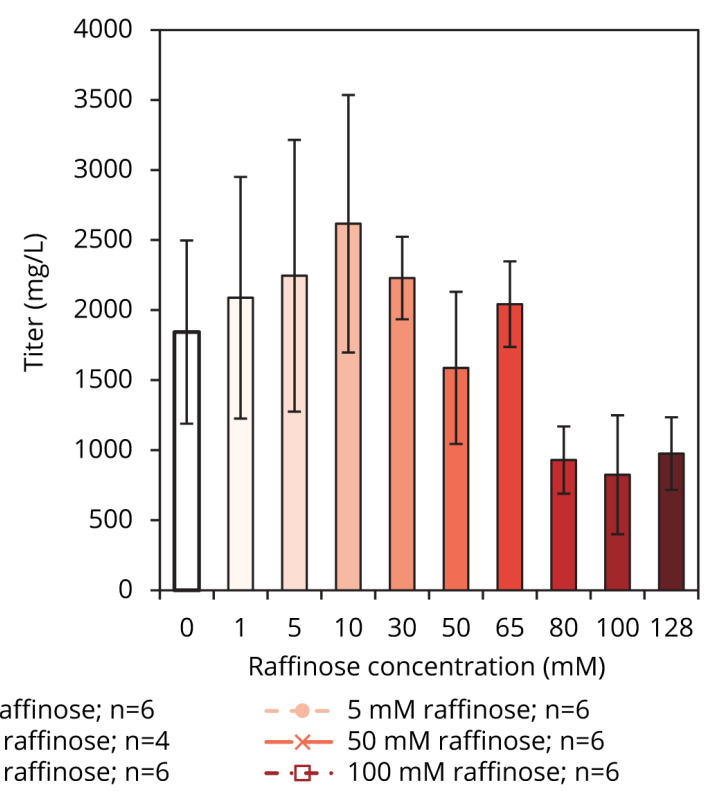

Figure 4.3 - (A) Viable cell densities of cell line 2 cultures supplemented with $0-128 \mathrm{mM}$ of raffinose. (B) Harvest titers (day 14) of cell line 2 cultures. All raffinose supplemented cultures were performed in replicates as indicated in legend of figure A in 96-DWP. The medium osmolality after raffinose addition was adjusted to $315 \mathrm{mOsm} / \mathrm{kg}$, which corresponds to the osmolality of the non-supplemented medium. 
media supplementation with raffinose was confirmed in lab-scale 3.5-L bioreactors (figure 4.5). $1.4 \%$ of $\mathrm{mAb}$ cell line 1 expressed contained HM. At a concentration of $15 \mathrm{mM}$ of raffinose in the medium prior to inoculation the HM proportion climbed to $2.9 \%$ and at $30 \mathrm{mM}$ to $6.7 \%$.

To validate the hypothesis that the raffinose supplementation is the main driver for the increase of $\mathrm{HM}$ and that the osmolality plays a minor role, the effect of raffinose supplementation was studied at three different osmolalities. HM significantly increased when supplementing the media with $30 \mathrm{mM}$ raffinose at all three osmolalities (figure 4.6). The media osmolality slightly affected its amplitude. At $300 \mathrm{mOsm} / \mathrm{kg}$ the raffinose addition resulted in $6.3 \% \mathrm{HM}$, at $315 \mathrm{mOsm} / \mathrm{kg} 6.8 \%$, and at the highest tested osmolality, $375 \mathrm{mOsm} / \mathrm{kg}, 7.1 \%$ were reached.

\subsubsection{Analysis of Nucleotides, Nucleotide Sugars and Transcriptomics}

Overall, the addition of raffinose had little effect on the nucleotide sugar (NS) levels (figure 4.7). One notices a slight decrease of UDP-GlcNAc, UDP-Glc and UDP-GalNAc at $100 \mathrm{mM}$ raffinose. In the control their concentrations amounted to $1.90,0.85$ and $0.69 \mathrm{fmol} /$ cell, whereas at $100 \mathrm{mM}$ raffinose they decreased to $1.47,0.73$ and $0.58 \mathrm{fmol} /$ cell, respectively. The intracellular abundance of UDP-Gal was higher at 50 and $100 \mathrm{mM}$ raffinose: 0.68 and 0.53 versus $0.40 \mathrm{fmol} / \mathrm{cell}$. On the other hand, ATP, CTP+CDP and UTP exhibited a downward trend with increasing raffinose levels, while ADP, GTP and UDP increased at intermediate concentrations and then began to decrease at high trisaccharide concentrations.

At $100 \mathrm{mM}$ raffinose, we observed changes in the gene expression of a variety of genes involved in the glycosylation pathway. As shown in table 4.1, $\beta$-1,4-galactosyltransferase 3 (GalT) gene was down-regulated by $\log _{2}-0.5142$, while galactosidase $\alpha$ gene was upregulated by $\log _{2} 0.52691$. Raffinose induced a gene upregulation of one of the most important enzymes involved in the glycosylation pathway, mannosyl ( $\alpha$-1,6-)-glycoprotein $\beta$-1,6-Nacetyl-glucosaminyltransferase (Mgat5) by $\log _{2} 0.33313$. Interestingly, both neuraminidase 1 and ST 8 -N-acetyl-neuraminide $\alpha$-2,8-sialyltransferase 6 genes were highly upregulated by $\log _{2} 0.87938$ and $\log _{2} 1.91184$, respectively. Finally, solute carrier genes were both up- and downregulated. The solute carrier family 35 , member A4 gene that codes the UDP-galactose transporter was downregulated by $\log _{2}-0.63845$.

\subsection{Discussion}

High throughput fed-batch experiments in 96-DWP proved to be a useful approach to screen a wide raffinose concentration range in the cell culture medium. Rather than an iterative approach of a couple of sequential experiments, the use of 96-DWP enabled to test two different cell lines at numerous supplement concentrations in a single experiment. This miniature-scale system was also suitable to optimize the concentration range and to develop the constant osmolality approach. In particular for cell line 2, the combination of the 96-DWP and the 


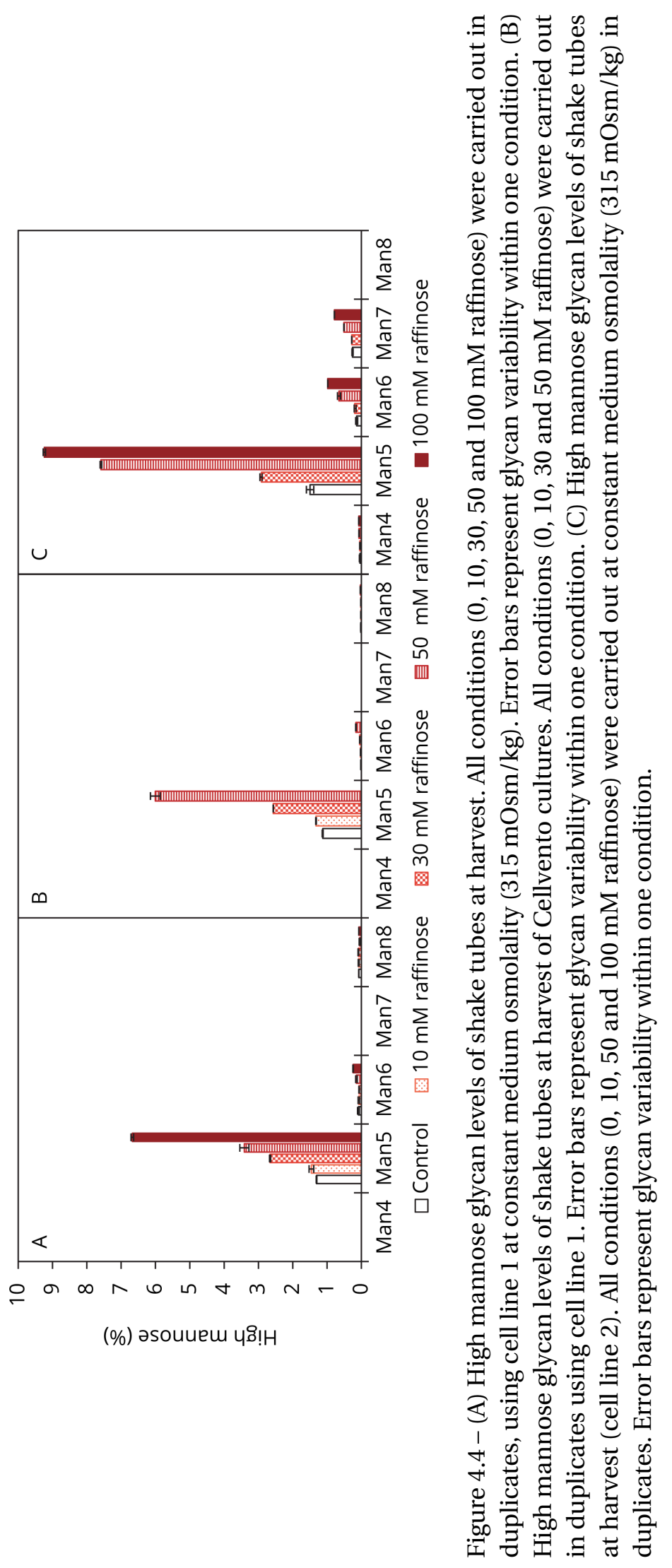




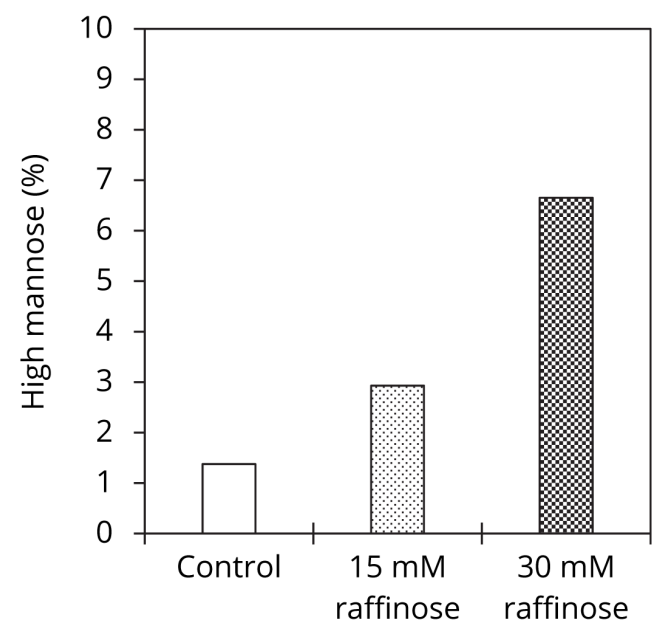

Figure 4.5 - High mannose levels in 3.5-L bioreactor runs with cell line 1 at $0 \mathrm{mM}$ (control), 15 and $30 \mathrm{mM}$ raffinose in the medium $(\mathrm{n}=1)$.

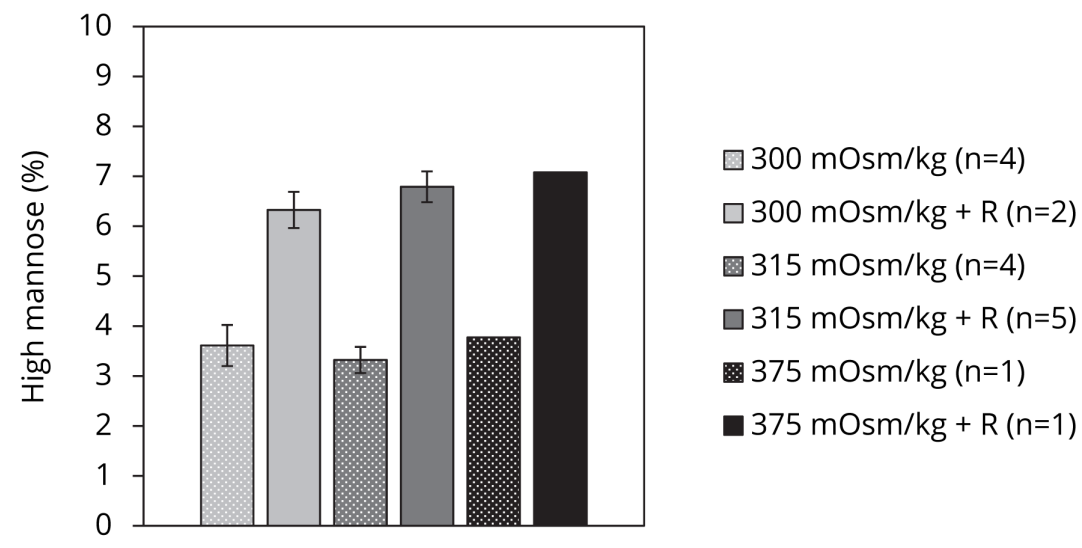

Figure 4.6 - High mannose level at three medium osmolalities (300, 315 and $375 \mathrm{mOsm} / \mathrm{kg}$ ) in the absence of raffinose and with $30 \mathrm{mM}$ raffinose supplementation $(+\mathrm{R})$ in cell line 2 cultures performed in 96-DWP. 
Table 4.1 - Expression of genes involved in the glycosylation pathway in ST supplemented with $100 \mathrm{mM}$ raffinose. All values are relative to the non-supplemented condition and expressed in $\log _{2}$-fold changes.

\begin{tabular}{llc}
\hline ID & Gene Name & Gene Expression Change (-) \\
\hline B3galt2 & $\beta$-1,3-galactosyltransferase 2 & no change \\
B3gat3 & $\beta$-1,3-glucuronyltransferase 3 & -0.36756 \\
B4galt3 & $\beta$-1,4-galactosyltransferase 3 & -0.51420 \\
Chpf & chondroitin polymerizing factor & -0.45785 \\
Chst11 & carbohydrate (chondroitin 4) sulfotransferase 11 & 0.50846 \\
Galk1 & galactokinase 1 & -0.50752 \\
Gla & galactosidase $\alpha$ & 0.52691 \\
Gns & glucosamine (N-acetyl)-6-sulfatase & 0.94486 \\
Hyal1 & hyaluronoglucosaminidase 1 & 0.63853 \\
Mgat5 & mannosyl ( $\alpha$-1,6-)-glycoprotein & 0.33313 \\
& $\beta$-1,6-N-acetyl-glucosaminyltransferase & \\
Neu1 & neuraminidase 1 (lysosomal sialidase) & 0.87938 \\
Ogt & O-linked N-acetylglucosamine & no change \\
& (GlcNAc) transferase & \\
Pigq & phosphatidylinositol glycan anchor & -0.35746 \\
& biosynthesis class Q & \\
Rpn2 & ribophorin II & -0.33632 \\
Slc35a4 & solute carrier family 35 member A4 & -0.63845 \\
Slc35d1 & solute carrier family 35 member D1 & 0.44870 \\
Slc35f2 & solute carrier family 35, member F2 & -0.48684 \\
Slc35f5 & solute carrier family 35 member F5 & 0.43458 \\
St8sia6 & ST8 $\alpha$-N-acetyl-neuraminide & 1.91184 \\
& $\alpha$-2,8-sialyltransferase 6 & \\
Ugcg & UDP-glucose ceramide glucosyltransferase & 0.58303 \\
\hline & &
\end{tabular}




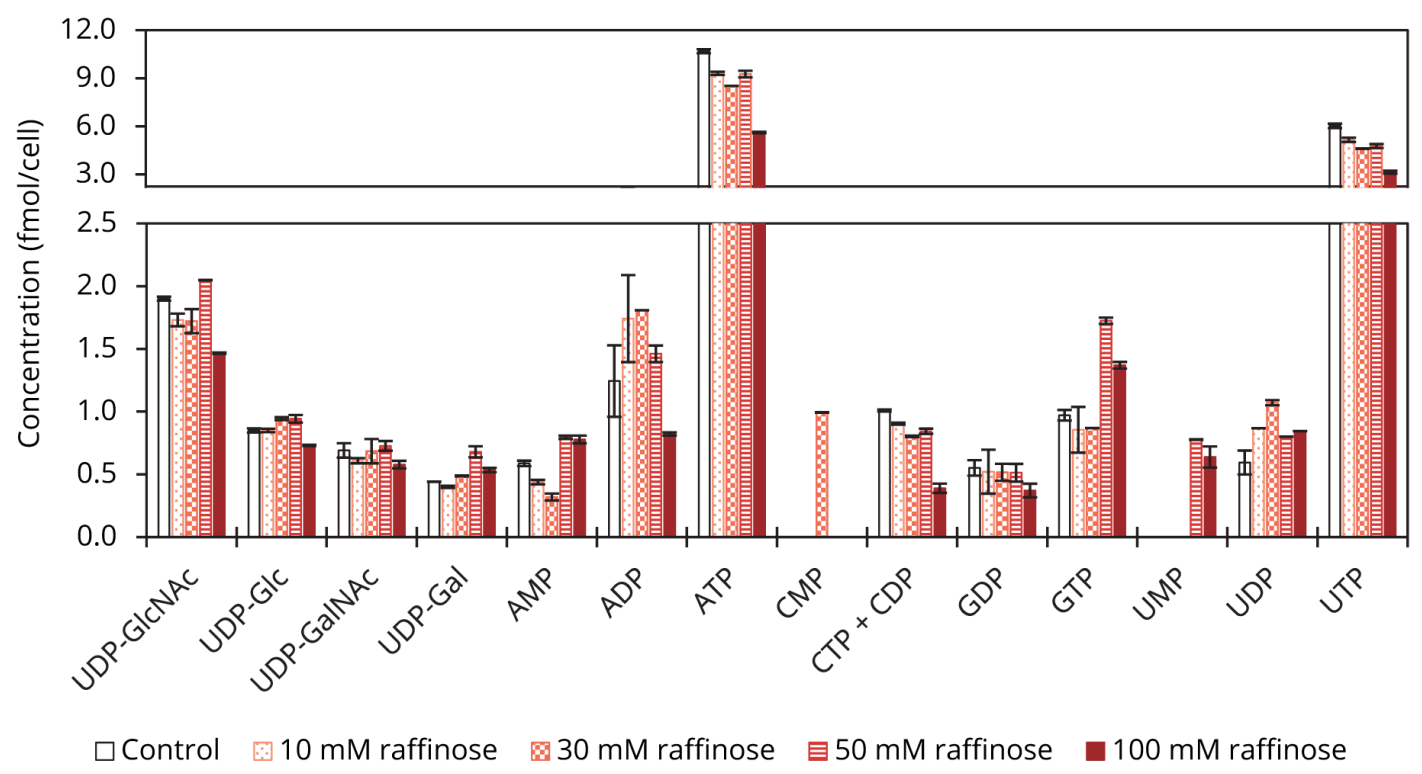

Figure 4.7 - Level of intracellular nucleotides and nucleotide sugars (cell line 1) in ST runs at $0 \mathrm{mM}$ (control), 15, 30, 50 and $100 \mathrm{mM}$ raffinose in the medium on culture day 3 . The error bars indicate the standard deviation of the technical duplicates.

development of the constant medium osmolality method enabled a substantial increase of the raffinose concentration, while limiting the effects on both cell growth and productivity. Up to $65 \mathrm{mM}$ of raffinose in the medium prior to inoculation resulted in comparable product titers to the non-supplemented control cultures. Some of the experiments were characterized by a high experimental variability of viable cell density and titer. Nonetheless, as the interest was directed towards the trends in function of the supplement concentration, the outcome of high throughput experiments largely outweighed the fluctuation within the replicates. Moreover, the observations were not only reproduced in larger cell-culture systems but more specific high mannose glycan data were generated, using the 2AB-UPLC method that allowed a greater resolution than CGE-LIF high throughput method. In ST at constant osmolality at all tested raffinose concentrations, a high mannose abundance of $11.1 \%$ was obtained at the most. Raffinose supplementation stands out from other well-known HM promoters, including kifunensine that mainly favors Man8 and $9^{91}$, whereas Man5 was the predominant species in both cell lines. Interestingly, cell line 1 exhibited mainly Man5 and 6. Man7 was not detected and Man8 was only present in tiny amounts. While Man5 was the main high mannose peak of cell line 2 also, considerably higher abundances of Man6 and 7 were observed in cell line 2. Similar effects resulted in cultures with both proprietary and commercial media. The effect of raffinose on $\mathrm{HM}$ abundance was confirmed in $\mathrm{pH}$, oxygen and $\mathrm{CO}_{2}$ controlled conditions in 3.5-L bioreactors. At both 15 and $30 \mathrm{mM}$ raffinose, a greater number of $\mathrm{HM}$ containing mAbs was expressed, reaching a 4.8-fold increase with respect the control. Hence, raffinose supplementation reproducibly favors HM in two different cell lines, $\mathrm{CHO}-\mathrm{S}$ and $\mathrm{CHO}-\mathrm{K} 1$, in different cell culture media and both in non-controlled and controlled cell culture systems. 
Moreover, the results highlight that, primarily, the presence of raffinose favors the formation of high mannose species. Increasing osmolality had a negligible effect on HM in the absence of raffinose in the cell culture medium. Contrariwise, at a concentration of $30 \mathrm{mM}$ of raffinose, we hypothesize that a minor synergistic effect between the raffinose supplementation and the media osmolality occurred. Despite this observation the major increase was due to raffinose, whereas the abundance of HM increased only marginally with increasing osmolality.

Raffinose barely influenced the intracellular nucleotide sugar levels. Slightly lower concentrations of UDP-GlcNAc, UDP-Glc and UDP-GalNAc resulted at $100 \mathrm{mM}$ raffinose. UDP-Gal exhibited an increasing tendency at 50 and $100 \mathrm{mM}$ raffinose, which may explain higher galactosylation levels. While intracellular NS pools varied only slightly, further studies should address the link of raffinose with the transport of NS into the Golgi apparatus. Nonetheless, higher trisaccharide concentrations correlated with decreasing levels of ATP, CTP+CDP and UTP. A correlation between nucleotide levels and metabolic changes was observed in carcinogenesis, where in particular ATP and UTP contents were significantly greater in cancer cells ${ }^{220}$. Likewise, raffinose supplementation most strongly affected intracellular ATP and UTP pools.

Transcriptomics analysis showed that raffinose supplementation influenced the expression levels of a number of glycosylation related genes. The expression of one of the most important enzymes, Mgat5, that plays a pivotal role in the regulation of the biosynthesis of glycoprotein oligosaccharides $^{221}$, was upregulated. GalT expression was down-regulated as well as the UDP-galactose transporter, solute carrier family 35 , member A4. This is surprising since galactosylation increased at higher raffinose concentration. Further investigations are required to pinpoint the underlying mechanism at the gene level and resulting real protein level, on the substrate transport into the Golgi apparatus and the GalT activity. Also, it seems that GalT3 may principally synthesize the first $\mathrm{N}$-acetyllactosamine unit of poly- $\mathrm{N}$-acetyllactosamine chains $^{222}$. The expression of the UDP-glucuronic acid and UDP-GalNAc transporter genes (gene: Slc35d1) ${ }^{223}$ was upregulated. Sialyltransferase (SialT) gene expression was highly upregulated, whereas the levels of sialic acid remained low (not shown). It is supposed that on one hand the down-regulation of the $\beta$-1,4-GalT 3 gene and the upregulation galactosidase $\alpha$ gene, and on the other hand, due to steric hindrance effects in the $\mathrm{CH} 2$-domain of the mAb, the considerably higher SialT gene expression still resulted in a negligible effect on the entire sialylation process. Although the enzyme was potentially present in higher concentrations, its accessibility was either unfavorable, or other unknown parameters hampered the attachment of sialic acid to the galactose moiety of the oligosaccharide backbone. Raffinose may also act as a GlcNAc transferase inhibitor, which may explain the predominant Man5 increase. Data from the cancer research further support this hypothesis. They exhibited competition of trisaccharides with the acceptor to bind to GlcNAc transferase ${ }^{224-226}$. 


\subsection{Conclusion}

Fed-batch cultures in high-throughput and lab-scale bioreactor systems supplemented with raffinose reproducibly favored the formation of high mannose glycans. The amount of high mannose species was proportional to the raffinose concentration in two different media and using two cell lines expressing different mAbs. While the presence of raffinose slightly affected nucleotide levels and to even smaller extent nucleotide sugar pools, it altered the expression levels of a variety of glycosylation related genes. In particular, GalT were downregulated, while SialT were strongly upregulated. Our results highlight the potential of cell culture medium supplementation to alter glycosylation patterns of recombinant proteins. Changing the environment the cells are cultured is a rather straightforward approach that allows to fine-tune within the potential of the selected cell line. 


\section{Chapter 5}

\section{Specific Inhibition of Galactosylation}

\subsection{Introduction}

The literature has repeatedly reported that galactosylation plays a role in effector functionality and immune responses. In a study comparing hypergalactosylated, degalactosylated and native IgG1 an impact on Fc $\gamma$ RIIIa binding was observed ${ }^{227}$. The hypergalactosylated variant promoted binding to the receptor, which may potentially induce changes in the antibody-dependent cell-mediated cytotoxicity (ADCC) activity, concluded the reasearchers. This relationship was recently confirmed: four hypergalactosylated IgG antibodies exhibited increased Fc $\gamma$ RIIIa binding compared to the control, and some of these variants also led to higher $\mathrm{ADCC}^{228}$. On the other hand, in-vitro testing demonstrated that the level of galactosylation inversely associated with ADCC activity ${ }^{229}$. While non-galactosylated and entirely galactosylated variants did not alter ADCC, the arm linkages of the mono-galactosylated species (FA2G1) predicted the immune response. Increased levels of the FA2G1 3-arm resulted in both reduced ADCC and reduced Fc $\gamma$ RIIIa binding, whereas the 6 -arm had a positive impact on ADCC. Other data depict how the presence of the galactose on the 6-arm enables additional hydrogen bonds between the sugar and the $\mathrm{CH} 2$ amino acid residues, and thus stabilizes the antibody. The greater stability may play a role in increasing the binding affinity ${ }^{230}$. There is also evidence that increased galactosylation levels enhance cell-dependent cytotoxicity (CDC). For instance, the FA2G1 glycoform of rituximab triggered a twofold stronger CDC response than the degalactosylated antibody ${ }^{67,200}$. Furthermore, like sialylation, galactosylation regulates inflammatory characteristics of IgG due to tertiary structure modifications and receptor interactions ${ }^{231,232}$. It has been described that rheumatoid arthritis was correlated with reduced galactosylation of $\operatorname{IgG}^{233,234}$. Interestingly, the degree of non-galactosylated IgG in the plasma decreases during pregnancy. The maternal immune system undergoes these changes to suppress immunological responses in order to tolerate the fetus ${ }^{235}$. Galactosylation, independent of sialylation, improved rheumatoid arthritis during pregnancy ${ }^{236}$. The symptoms in women suffering from rheumatoid arthritis worsen after delivery, coinciding 
with a decrease of di-galactosylated biantennary glycans.

Given the important role of galactosylation, it is not a surprise that in the last two decades both academia and industry have been developing technologies to alter the galactosylation pattern of recombinant proteins. Targeted metabolic engineering approaches have been successfully applied to tune $\mathrm{mAb}$ glycan profile in cell culture. $\mathrm{N}$-acetylglucosamine supplementation in cell culture favored non-galactosylated glycans (FA2) ${ }^{204}$. Exact and specific control of antibody galactosylation was achieved by feeding of uridine, manganese chloride and galactose, primarily shifting FA2 to FA2G1 species ${ }^{52}$. Ammonium supplementation increased the transGolgi $\mathrm{pH}$, thus reducing galactosyltransferase activities, which yielded lower galactosylation levels ${ }^{237}$. It was found that high ammonium concentration in the supernatant $\left(10 \mathrm{mM} \mathrm{NH}_{4} \mathrm{Cl}\right)$ diminished galactosyltransferase gene expression levels ${ }^{126}$. While supplementation of uridine, manganese and galactose offers a wide range of galactosylation enhancement, which proves to be sufficient in the frame of the quality modulation exercise of new biological entities and biosimilars, a limited number of effective compounds to decrease galactosylation during cell culture is available. Ammonium does work, but it is not entirely specific, and more importantly, high ammonium levels exhibit detrimental effects on cell culture performance ${ }^{238}$.

Aiming to develop alternative and more specific ways to inhibit galactosylation, the work presented by Rillahan et al. ${ }^{112}$ was used as inspiration. The researchers used membranepermeable fluorinated analogs of sialic acid and fucose. They suggested that after entering into the cytosol, those precursors would convert into the corresponding nucleotide sugars, and at a the same time, prevent de novo synthesis of the natural substrates by feedback inhibition. Cell-culture media supplementation of 2F-peracetyl fucose and 3F-neuramic acid specifically and strongly inhibited fucosyl- and sialyltransferases. Likewise, a targeted metabolic engineering approach is presented hereafter, using fluorinated analogs of galactose. Two anomers, $\alpha$-2F-peracetyl-galactose and $\beta$-2F-peracetyl-galactose were added into the cell culture medium at the beginning or by means of the feed throughout the culture at various time points. The effect of the supplements was first assessed in high-throughput fed-batch cell culture experiments in shaken 96-deepwell plates and then promising conditions repeated, scaling up into the more robust shake-tube model. Additionally, as part of a larger component screening exercise, spermine, a natural polyamine, and L-ornithine, a non-coding amino acid involved in the urea cycle, were found to inhibit galactosylation. This work shows that the fluorinated galactose analogs reproducibly and specifically reduced the level of galactosylation in two fed-batch cell culture systems 96-deepwell plates and shake tubes, using two distinct $\mathrm{CHO}$ cell lines. Additional approaches calling on medium supplementation with spermine or L-ornithine are outlined as well. 


\subsection{Materials and Methods}

\subsubsection{Inoculum Preparation}

Two recombinant cell lines were used in the frame of this study. A CHO-S derived clonal cell line expressing a human monoclonal IgGl antibody (cell line A) and a CHO-K1 derived clonal cell line expressing a humanized monoclonal IgG1 antibody (cell line B). Cells were first expanded in shake tubes or shake bottles in Merck in-house medium containing methionine sulfoximine (MSX) in multiple passages every 2-3 days, diluting to 0.2 or $0.3 \times 10^{6}$ viable cells $/ \mathrm{mL}$ for at least 14 days. The tubes were maintained in a shaker incubator at $36.5{ }^{\circ} \mathrm{C}, 5 \%$ $\mathrm{CO}_{2}, 80 \%$ humidity and $320 \mathrm{rpm}$ agitation (ISF1-X, Adolf Kühner, Birsfelden, Switzerland or Multitron Cell, Infors HT, Bottmingen, Switzerland).

\subsubsection{Cell Culture Conditions}

The high-throughput fed-batch cell culture was performed on a robotic liquid handling platform (Biomek FX, Beckman Coulter, Brea, CA). Exponentially growing CHO-S cells were seeded into a shaking 96-deepwell plate (DWP) filled with Merck in-house medium enriched with different supplements at the concentrations indicated in table 5.1 in the absence of MSX at a viable cell density of $0.20 \times 10^{6}$ viable cells $/ \mathrm{mL}$. The plates were incubated with vented lids to minimize evaporation in a shaker incubator at $36.5{ }^{\circ} \mathrm{C}, 5 \% \mathrm{CO}_{2}, 90 \%$ humidity and $320 \mathrm{rpm}$ agitation (ISF1-X, Adolf Kühner, Birsfelden, Switzerland) for 14 days. 400 g/L glucose solution, chemically-defined feed containing over 30 components and alkaline amino acid solution were added on day 3, 5, 7, 10 and 12. Prior to each feeding and at the end of the culture on day 14 , samples $(\leq 40 \mu \mathrm{L}$ ) were drawn for growth and viability assessment and product titer quantification.

The confirmation runs were conducted in TPP® TubeSpin bioreactor tubes (referred to shake tubes or $S T$ ) filled with Merck in-house supplement enriched medium according to table 5.1 in the absence of MSX. Exponentially growing CHO-S cells were seeded at a viable cell density of $0.30 \times 10^{6}$ viable cells $/ \mathrm{mL}$ and exponentially growing CHO-K1 cells at $0.20 \times 10^{6}$ viable cells $/ \mathrm{mL}$. The tubes were incubated in a shaker incubator at $36.5^{\circ} \mathrm{C}, 5 \% \mathrm{CO}_{2}, 80 \%$ humidity and $320 \mathrm{rpm}$ agitation (ISF1-X, Adolf Kühner, Birsfelden, Switzerland) for 14 days. Chemically defined feed containing over 30 components and alkaline amino acid solution were added on day 3, 5, 7 and 10 , while the $400 \mathrm{~g} / \mathrm{L}$ glucose solution was added on these days and on day 12 in addition. Prior to each feeding and at the end of the culture (day 14), aliquots ( $\leq 2.5 \mathrm{~mL}$ ) were taken for viable cell counting, extracellular metabolite profiling and product titer determination.

To evaluate the potential of the supplement addition during the culture as part of the feed rather than at the beginning of the culture in the medium, shake tubes were used. Exponentially growing CHO-S cells were inoculated at $0.30 \times 10^{6}$ viable cells $/ \mathrm{mL}$ in proprietary 
medium only (in the absence of MSX). The ST were incubated in a shaker incubator at $36.5^{\circ} \mathrm{C}$, $5 \% \mathrm{CO}_{2}, 80 \%$ humidity and $320 \mathrm{rpm}$ agitation (ISF1-X, Adolf Kühner, Birsfelden, Switzerland) for 14 days. Chemically defined feed containing over 30 components and alkaline amino acid solution were added on day 3, 5, 7 and 10 . The $400 \mathrm{~g} / \mathrm{L}$ glucose solution was added on day 3, 5, 7 and 10 and on day 12 too. On top of it, $\beta$-2F-peracetyl-galactose (AX Molecules) was added, starting on day 3 , day 5 or day 7 until day 10 according to table 5.2. At each feed addition, the concentration of the galactose analog in the supernatant increased by $100 \mu \mathrm{M}$. Prior to each feeding and at the end of the culture (day 14$)$, aliquots $(\leq 2.5 \mathrm{~mL})$ were removed for viable cell counting, extracellular metabolite profiling and product titer quantification.

\subsubsection{Analytical Methods for Cell Culture Performance}

Growth and viability assessment of 96-DWP cultures was performed on a Guava easyCyte (Merck Life Science, Darmstadt, Germany), while for shake tube and 3.5-L bioreactor cultures a Vi-Cell analyzer (Beckman Coulter, Brea, CA) was used. The product titer of the 96-DWP cultures was analyzed on day 14 by an Octet ${ }^{\circledR}$ (forteBIO, Menlo Park, CA), using Protein A sensors. Each sample was diluted 20-40 times into a dilution buffer ( $\mathrm{PBS} \mathrm{pH}=7.4$, BSA 1 $\mathrm{g} / \mathrm{L}$, Tween 20 at $0.02 \%$ ). The sensors were regenerated with aqueous buffer containing 10 $\mathrm{mM}$ glycine- $\mathrm{HCl}$ at $\mathrm{pH} 1.5$ and neutralized with the dilution buffer. Titer quantification of samples from shake tubes collected on day 14 was performed, using a Biacore C instrument (GE Healthcare, Waukesha, WI).

Table 5.1 - Concentrations of glycosylation modulating compounds in the cell culture medium prior to inoculation.

\begin{tabular}{llll}
\hline Compound & Scale & Concentration Ranges & Supplier \\
\hline$\alpha$-2F-peracetyl-galactose & $96-\mathrm{DWP}$ & $0-200 \mu \mathrm{M}$ & Biosynth \\
& ST & $0-90 \mu \mathrm{M}$ & Biosynth \\
$\beta$-2F-peracetyl-galactose & $96-\mathrm{DWP}$ & $0-200 \mu \mathrm{M}$ & AX Molecules \\
& ST & $0-60 \mu \mathrm{M}$ & AX Molecules \\
Ammonium & ST & $0-10 \mathrm{mM}$ & Merck \\
Spermine & $96-\mathrm{DWP}$ & $0-200 \mu \mathrm{M}$ & Sigma Aldrich \\
L-ornithine & $96-\mathrm{DWP}$ & $0-15 \mathrm{mM}$ & Sigma Aldrich \\
\hline
\end{tabular}

Table 5.2 - Feeding regime of ST feed optimization experiments.

\begin{tabular}{|c|c|c|c|c|c|c|}
\hline Experiment & Day 3 & Day 5 & Day 7 & Day 10 & Day 12 & Day 14 \\
\hline $\begin{array}{l}\text { CD feed \& amino acid solution; all } \\
\text { Glucose solution; all } \\
\beta \text {-2F-p-gal; feed day } 3 \\
\beta \text {-2F-p-gal; feed day } 5 \\
\beta \text {-2F-p-gal; feed day } 7\end{array}$ & $\begin{array}{l}\mathbf{\square} \\
\mathbf{\square}\end{array}$ & $\begin{array}{l}\square \\
\square \\
\square \\
\square\end{array}$ & $\begin{array}{l}\mathbf{\square} \\
\mathbf{\square} \\
\mathbf{\square}\end{array}$ & $\begin{array}{l}\mathbf{u} \\
\mathbf{\square} \\
\mathbf{\square} \\
\mathbf{\square}\end{array}$ & च & \\
\hline
\end{tabular}




\subsubsection{Glycan Analysis}

At the end of each 96-DWP and ST fed-batch experiment (day 14), the supernatant was purified on small-scale affinity columns (PhytipsVR, PhyNexus, San Jose, CA), eluting in $20 \mathrm{mM}$ citric acid, $20 \mathrm{mM} \mathrm{PO}_{4}^{3-}$ buffer. The samples were neutralized in $0.5 \mathrm{M}$ Tris. The $\mathrm{N}$-glycosylation pattern of the 96-DWP eluates was analyzed by capillary gel electrophoresis with laser-induced fluorescence detection (CGE-LIF, DNA genetic analyzer 3130XL, Life Technologies, Darmstadt, Germany). The ST eluates of cell line B were analyzed by Ultra Performance Liquid Chromatography-2-amino-benzamide labelling technique (2AB-UPLC). The $100 \mathrm{~mm}$ column (in length) was supplied by Waters Corporation, Milford, MA, USA. Both individual glycans and glycan groups are presented in section 5.3. The grouping calculations were performed according to table 5.3 for CGE-LIF results and table 5.4 for 2AB-UPLC data.

Table 5.3 - Glycan grouping calculation for CGE-LIF data.

\begin{tabular}{ll}
\hline Glycan & Sum of glycan peaks \\
\hline $\mathrm{HM}(\%)$ & $=(\mathrm{M} 5+\mathrm{FA} 1+\mathrm{FA} 2 \mathrm{G} 2 \mathrm{~S}[6] 1 \mathrm{NGNA})(\%)+(\mathrm{M} 6+\mathrm{FA} 2 \mathrm{G} 2 \mathrm{aG} 1 \mathrm{~S} 1)(\%)+\mathrm{M} 7(\%)$ \\
$\mathrm{AF}(\%)$ & $=\mathrm{A} 2(\%)$ \\
$\mathrm{Fuc}(\%)$ & $=\mathrm{FA} 1+\mathrm{FA} 2(\%)+\mathrm{FA} 2 \mathrm{G} 1(\%)+\mathrm{FA} 2 \mathrm{G} 2(\%)$ \\
$\mathrm{Gal}(\%)$ & $=\mathrm{FA} 2 \mathrm{G} 1(\%)+\mathrm{FA} 2 \mathrm{G} 2(\%)$ \\
\hline
\end{tabular}

Table 5.4 - Glycan grouping calculation for 2AB-UPLC data.

\begin{tabular}{lll}
\hline Glycan & Sum of glycan peaks \\
\hline $\mathrm{HM}(\%)$ & $=\mathrm{M} 4(\%)+\mathrm{M} 5(\%)+\mathrm{M} 6(\%)+\mathrm{M} 7(\%)$ \\
$\mathrm{AF}(\%)$ & $=\mathrm{A} 0(\%)+\mathrm{A} 1(\%)+\mathrm{A} 2(\%)$ \\
$\mathrm{Fuc}(\%)$ & $=\mathrm{FA} 2(\%)+\mathrm{FA} 2 \mathrm{G} 1(\%)+\mathrm{FA} 2 \mathrm{G} 2(\%)$ \\
$\mathrm{Gal}(\%)$ & $=\mathrm{FA} 2 \mathrm{G} 1(\%)+\mathrm{FA} 2 \mathrm{G} 2(\%)+(\mathrm{FA} 1[3] \mathrm{G} 1+\mathrm{A} 2[6] \mathrm{G} 1)(\%)$ \\
\hline
\end{tabular}

\subsection{Results}

\subsubsection{F-peracetyl-galactose}

The effect of the fluorinated galactose analog $\alpha$-2F-peracetyl-galactose on the level of galactosylation of cell line A was assessed in 96-DWP. The wells filled with cell culture medium were supplemented with $\alpha$-2F-p-galactose to reach a final concentration of 0-200 $\mu \mathrm{M}$. Figure 5.1 shows the viable cell densities, viabilities throughout the culture, and the product titers on day 10 and at harvest (day 14).

Despite the rather large variability of the viable cell density trends were visible (figure 5.1A). Supplement concentrations between 1 and $30 \mu \mathrm{M}$ enhanced cell growth, resulting in a higher 


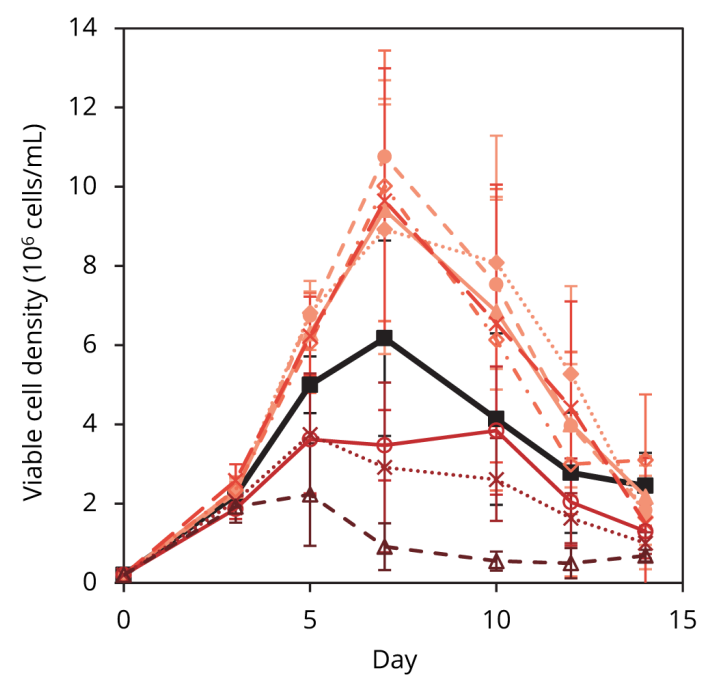

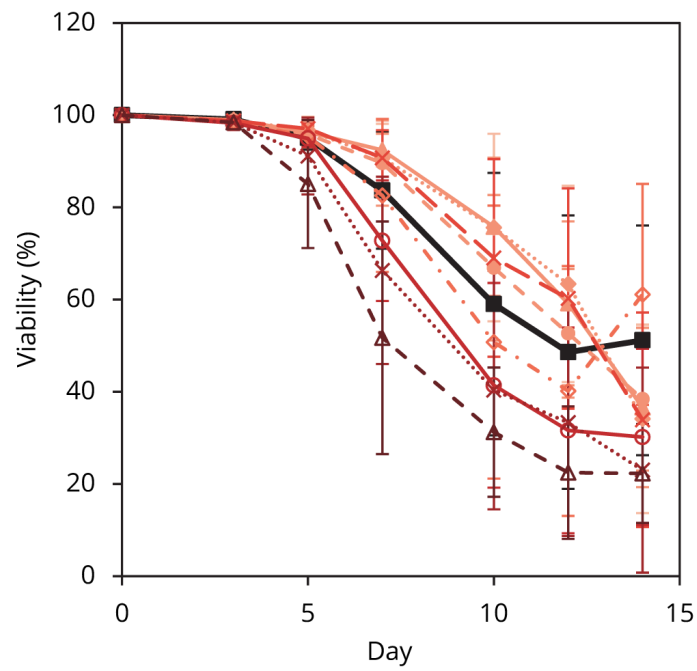

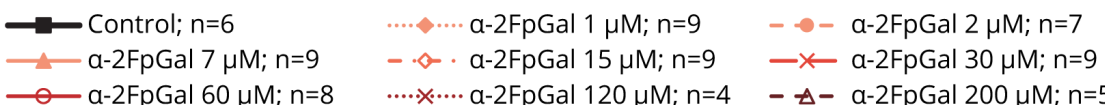

C

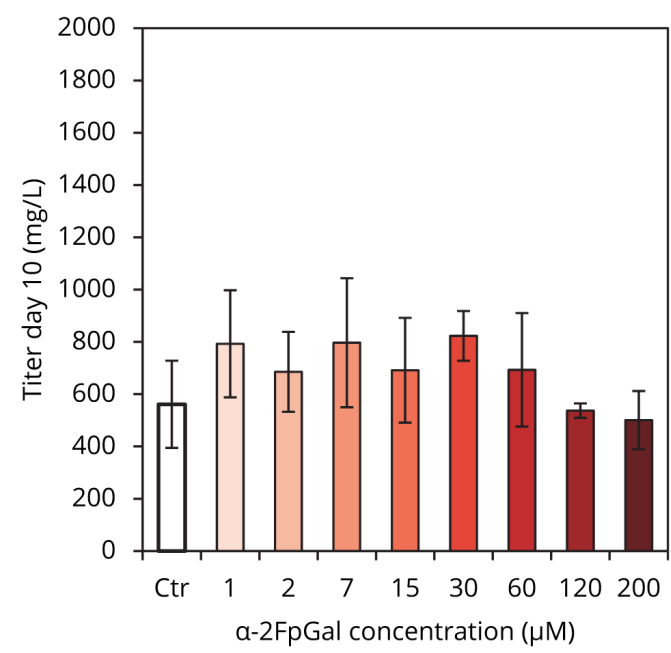

D

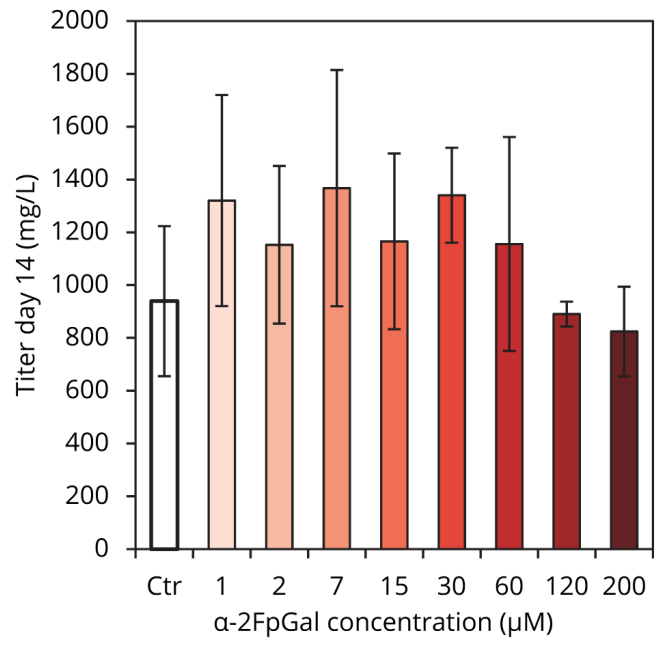

Figure 5.1 - (A) Viable cell densities of cell line A cultures supplemented with $0-200 \mu \mathrm{M}$ of $\alpha$-2F-peracetyl-galactose. (B) Viabilities. (C) Product titers on day 10. (D) Product titers in the harvest on day 14. The number of replicates of each condition is indicated in chart A. All points are mean values of the corresponding replicates and the error bars report the standard deviation of the replicates. 
peak cell density $\left(8.9-10.8 \times 10^{6} \mathrm{VC} / \mathrm{mL}\right)$ than in the control $\left(6.2 \times 10^{6} \mathrm{VC} / \mathrm{mL}\right)$. Although these values were higher than the control, one should notice that the error between the replicates of each concentration between 1 and $30 \mu \mathrm{M}$ on day 7 fluctuated between 20 and $40 \%$. Hence, due to this variability, $\alpha$-2F-p-galactose did not considerably impact cell growth up to $30 \mu \mathrm{M}$. At higher concentrations, the presence of the galactose analog inhibited cell growth, shifting the maximum peak cell density to day 5 . At $60 \mu \mathrm{M}$ it reached $3.6 \times 10^{6} \mathrm{VC} / \mathrm{mL}$ and at the highest concentration it further decreased to $2.2 \times 10^{6} \mathrm{VC} / \mathrm{mL}$. As a consequence, at 60,120 and $200 \mu \mathrm{M}$ the viabilities strongly decreased with time and ended at $\leq 30 \%$ (figure $5.1 \mathrm{~B}$ ). As presented in figures 5.1C and D, no clear titer trend was observed both on day 10 and 14 . The error bars indicate that the variability was important and no significant antibody production reduction resulted at all the tested concentrations. Titers at harvest ranged between 824 and $1367 \mathrm{mg} / \mathrm{L}$.

The control culture yielded $10 \%$ galactosylated species. Figure 5.2A shows that $\alpha$-2F-p-galactose reduced the attachment of terminal galactose on the FA2 entity. Substantial reductions resulted in cultures containing 30,60, 120 and $200 \mu \mathrm{M} \alpha$-2F-p-galactose. The absolute change amounted to $-1.3,-2.8,-3.5$, and $-4.8 \%$, respectively. Changes for high mannose, afucosylated and fucosylated species were small and in light of the analytical variability can be neglected. According to figure 5.2B, the level of A2 forms remained unchanged. FA2G1, the predominant galactosylated form, declined by $-1.1,-2.5,-3.1$, and $-4.4 \%$, respectively. As for the digalactosylated form, FA2G2, the change resulted in $-0.17,-0.30,-0.33,-0.38 \%$, respectively at 30, 60, 120 and $200 \mu \mathrm{M} \alpha$-2F-p-galactose.

A

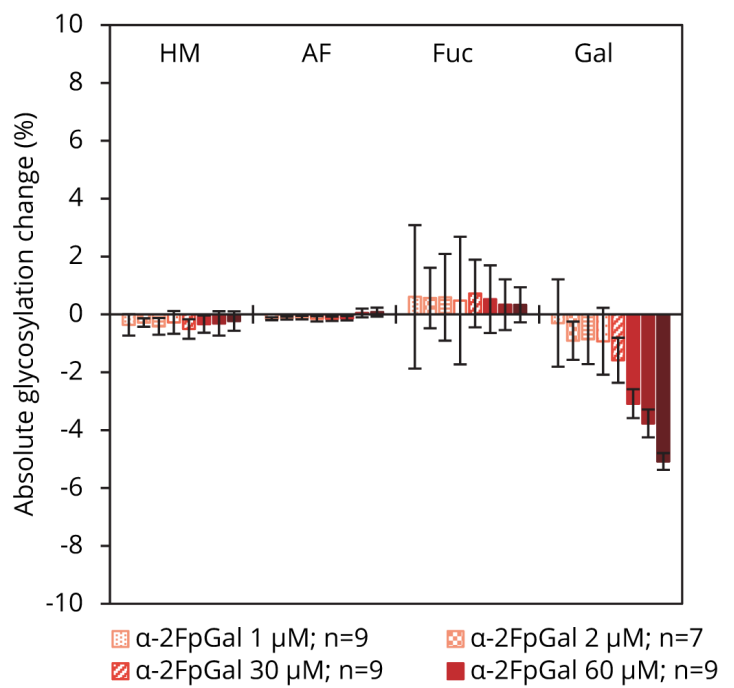

B

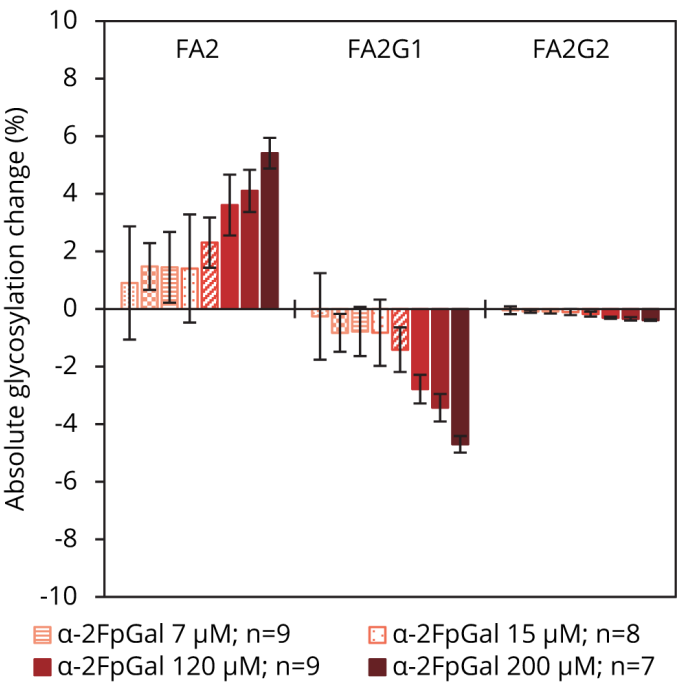

Figure 5.2 - (A) Absolute change of the overall glycosylation pattern compared to the control in function of the $\alpha$-2F-p-galactose concentration in medium. (B) Absolute change of galactosylation compared to the control in function of the $\alpha$-2F-p-galactose concentration in medium. All points are mean values of the corresponding replicates analyzed by CGE-LIF and the error bars report the standard deviation of the replicates. 
In the case of the fluorinated sialic acid analogs, the position of the fluorine affected the inhibition level of the corresponding glycan transferase ${ }^{112}$. Hence, the next step aimed to study the effect of the $\beta$-anomer of 2F-p-galactose at the same concentrations as for the $\alpha$-anomer. The control culture reached a maximum cell density of $6.3 \times 10^{6} \mathrm{VC} / \mathrm{mL}$ on day 5 (figure 5.3A). At concentrations beyond $60 \mu \mathrm{M}$ growth inhibitory effects began to appear. At highest concentration $(200 \mu \mathrm{M})$, the peak settled at $1.0 \times 10^{6} \mathrm{VC} / \mathrm{mL}$. Similarly to the $\alpha$ anomer, no clear titer trend was apparent (figure 5.3B). In general, the variability within one concentration range was important, which shows the need to confirm the observed trends at larger scale.

One more time, the galactosylation decrease was proportional with the $\beta$ - $2 \mathrm{~F}$-p-galactose concentration. According to figure 5.4A, 30, 60, 120, and $200 \mu \mathrm{M}$ cut back the overall galactosylation levels by $-2.2,-4.2,-5.4$, and $-6.7 \%$, respectively. The monogalactosylated glycan was the main contributor once again. It respectively decreased by $-2.0,-3.8,-5.0$, and $-6.2 \%$, while the entirely galactosylated form (FA2G2) slightly varied from $-0.23,-0.41,-0.44$ to $-0.53 \%$ (figure $5.4 \mathrm{~B}$ ). Both anomers affected the cell culture performance and the galactosylation inhibition in a similar manner in 96-DWP.

At this stage, the 2F-p-galactose supplementation was repeated in shake tubes to verify the reproducibility of the 96-DWP results and to test the response when culturing a different cell line. Figure 5.5A shows the viable cell density of cell line A cultures in ST at 30, 60 and $90 \mu \mathrm{M} \alpha$-2F-p-galactose in the medium. The control culture reached the highest density on day 7 , climbing up to $19.5 \times 10^{6} \mathrm{VC} / \mathrm{mL}$. The $\alpha$-galactose analog supplementation exhibited no growth inhibitory effect in the first part of the culture. Nonetheless, cell densities were slightly lower from day 10 until the end of the culture. On day 14 , the control cultures were harvested at a density of $11.5 \times 10^{6} \mathrm{VC} / \mathrm{mL}$. The supplemented cultures were in the range of 8.4 to $9.9 \times 10^{6} \mathrm{VC} / \mathrm{mL}$. Experiments with $60 \mu \mathrm{M} \beta$-2F-peracetyl-galactose peaked considerably lower at $12.8 \times 10^{6} \mathrm{VC} / \mathrm{mL}$. The data points before and after were however comparable to the $\alpha$-anomer and the measured peak cell density was lower than the cell counts on days 5 and 10 . Moreover, the viable cell density was higher than in the $\alpha$-cultures. Viabilities were comparable on day 7 (figure 5.5B). It is therefore possible that this difference was rather due to an analytical artefact, stemming from the imaged based cell fluorescent analyzer. This hypothesis is further supported by the fact that protein concentrations in the supernatant of the $\beta$-2F-p-galactose containing ST were comparable to the control at culture days 5, 7, 10, 12 and 14, yielding $2225 \mathrm{mg} / \mathrm{L}$. Likewise, the product titers of the $\alpha$-anomer were comparable at all time points, attaining at the end of the culture 2115, 2050, 2130, $2225 \mathrm{mg} / \mathrm{L}$, respectively, in the control, at 30, 60 and $90 \mu \mathrm{M}$. Unlike 96-DWP, in ST no detrimental effect on cell culture performance was observed in the entire concentration range between 0 and $90 \mu \mathrm{M} \alpha$-2F-p-galactose and at $60 \mu \mathrm{M} \beta$-2F-p-galactose. In comparison, ammonium, a well-known galactosylation inhibitor, hampered cell growth and viability. The addition of the ammonium salt into the cell culture broth on day 5 caused reduced cell densities, viabilities as well as product titers (-15\% on day 14). 
A

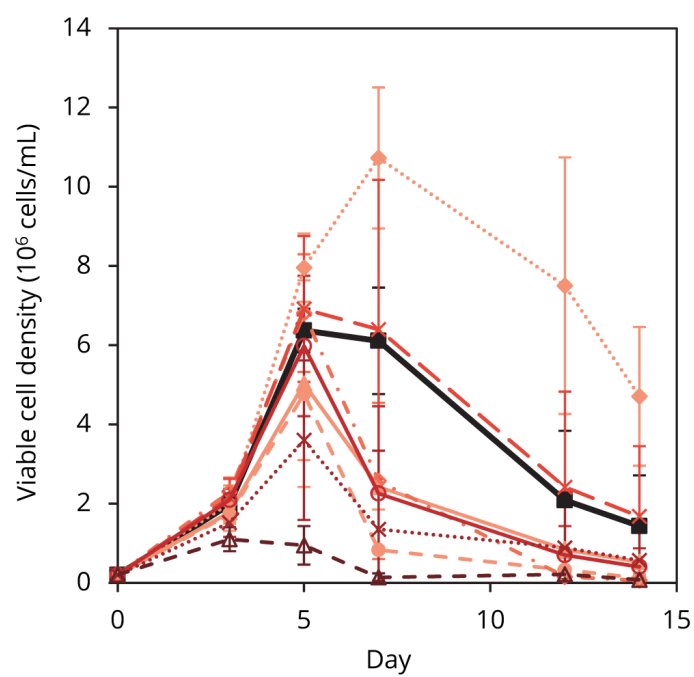

B

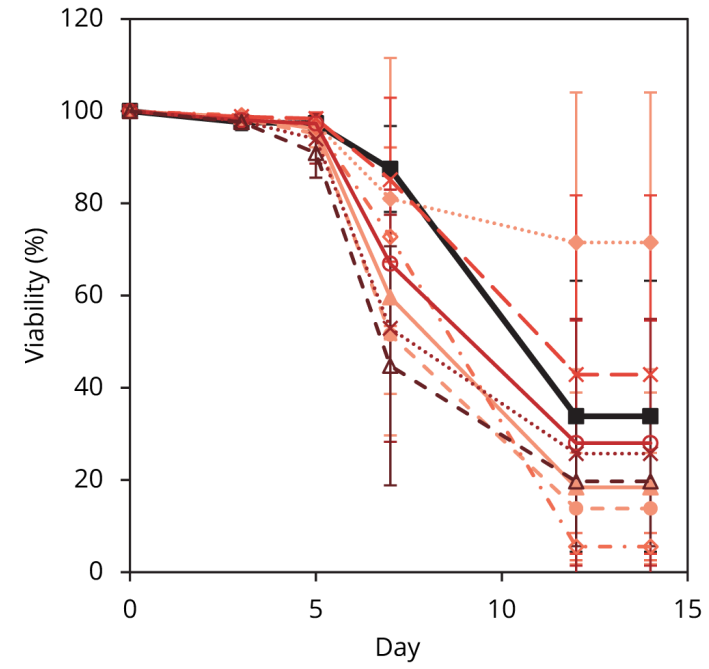

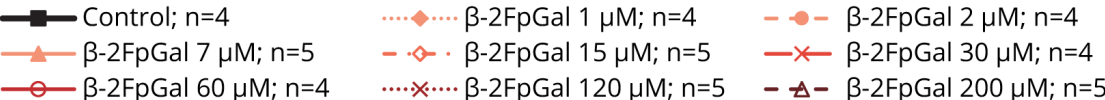

C

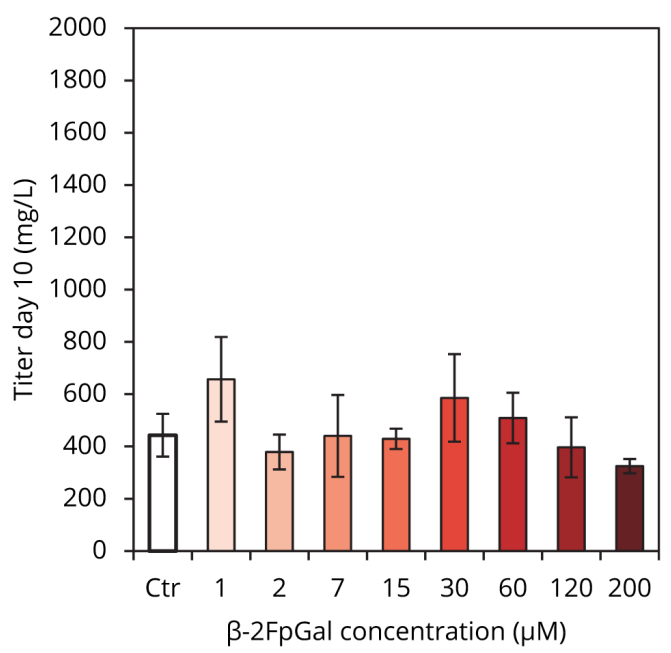

D

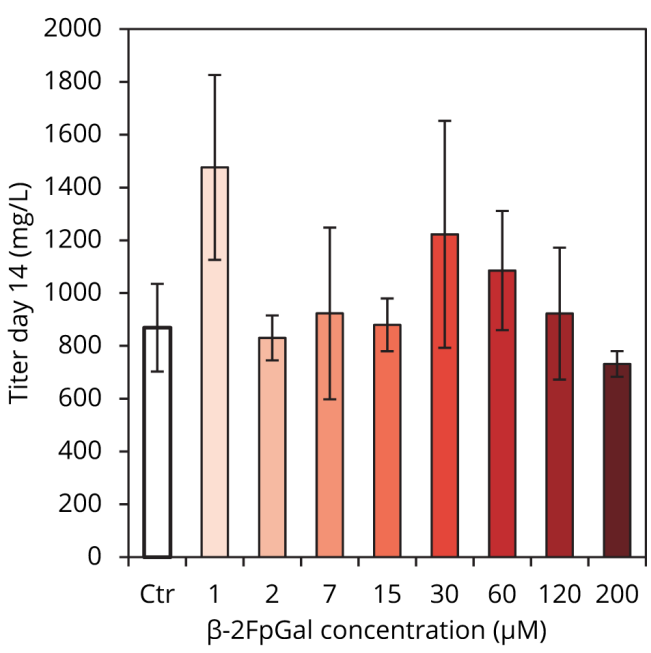

Figure 5.3 - (A) Viable cell densities of cell line A cultures supplemented with 0-200 $\mu \mathrm{M}$ of $\beta$ 2F-peracetyl-galactose. (B) Harvest titer (day 14). The number of replicates of each condition is indicated in chart A. All points are mean values of the corresponding replicates and the error bars report the standard deviation of the replicates. 
A

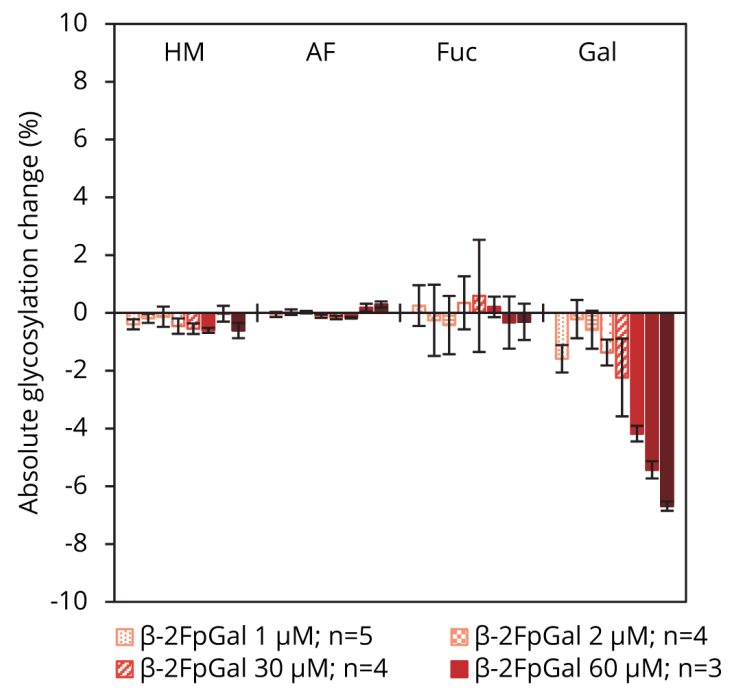

B

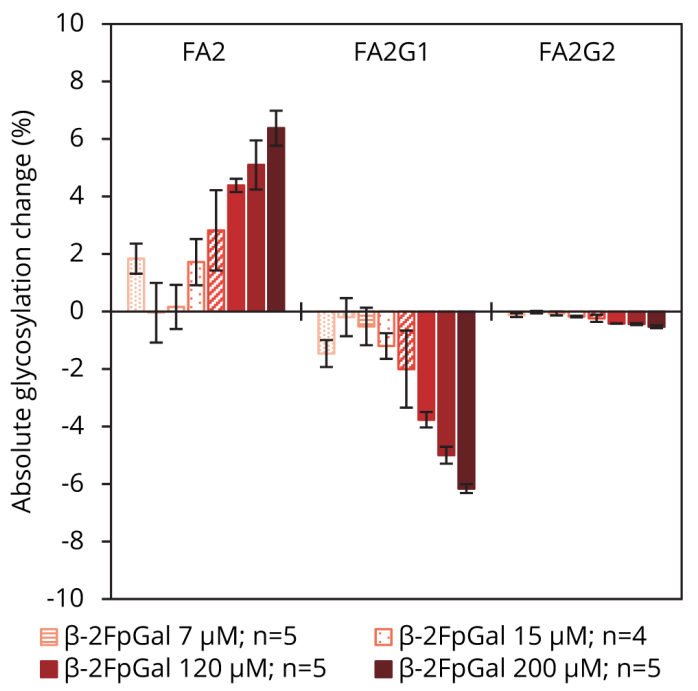

Figure 5.4 - (A) Absolute change of the overall glycosylation pattern compared to the control in function of the $\beta$-2F-p-galactose concentration in medium. (B) Absolute change of galactosylation compared to the control in function of the $\beta$-2F-p-galactose concentration in medium. All points are mean values of the corresponding replicates analyzed by CGE-LIF and the error bars report the standard deviation of the replicates.

According to figure 5.6 the galactosylation inhibiting effects of the galactose analog observed in 96-DWP were confirmed in ST. The total level of galactosylation decreased by $-2.0,-4.6$ and $-4.5 \%$ at respectively 30,60 and $90 \mu \mathrm{M} \alpha$-2F-peracetyl-galactose and by -5.0 with $60 \mu \mathrm{M}$ $\beta$-2F-peracetyl-galactose. The monogalactosylated form changed by $-1.9,-4.3,-4.3 \%$ at respectively 30, 60 and $90 \mu \mathrm{M} \alpha$-2F-peracetyl-galactose and by $-4.7 \%$ in the presence of the $\beta$-anomer. The entirely galactosylated glycoform slightly decreased between -0.1 and $-0.3 \%$. Overall, the performance of the two anomers was comparable. One could argue that the $\beta$ form displayed a slight tendency towards enhanced inhibition. On the other hand, ammonium supplementation further enhanced the inhibition effect, yielding a $-7.2 \%$ reduction. Nevertheless, the use of 2F-p-galactose is worthwhile due to its high specificity which limits the effect on other glycan species. The ammonium concentration was correlated with increases $\geq 1 \%$ of high mannose species, afucosylated and fucosylated species, revealing its lower degree of specificity.

The medium supplementation of $\alpha$-2F-peracetyl-galactose was also evaluated in cell line B. As figure 5.7A highlights, the overall viable cell densities of the 30 and $60 \mu \mathrm{M} \alpha$-2F-p-galactose ST cultures were comparable to the control, which reached a maximum cell density of $11.2 \times 10^{6}$ $\mathrm{VC} / \mathrm{mL}$ on day 7 . A level of $90 \mu \mathrm{M}$ led to reduced cell growth, peaking at $10.0 \times 10^{6} \mathrm{VC} / \mathrm{mL}$. The viabilities of the entire supplement concentration range were comparable (figure 5.7B). Intermediated concentrations ( 30 and $60 \mu \mathrm{M}$ ) kept the productivity unchanged (figure 5.7C). The highest inhibitor concentration entailed a little titer reduction. At harvest (day 14), it 
A

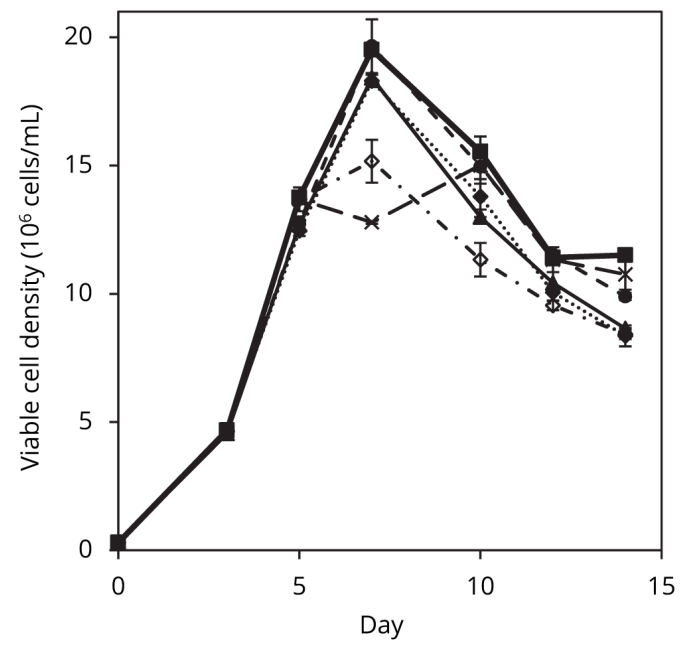

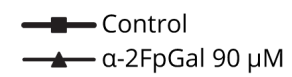

$\cdots \bullet \cdot \cdots$ a-2FpGal $30 \mu \mathrm{M}$

- $\cdot \beta$-2FpGal $60 \mu \mathrm{M}$

C

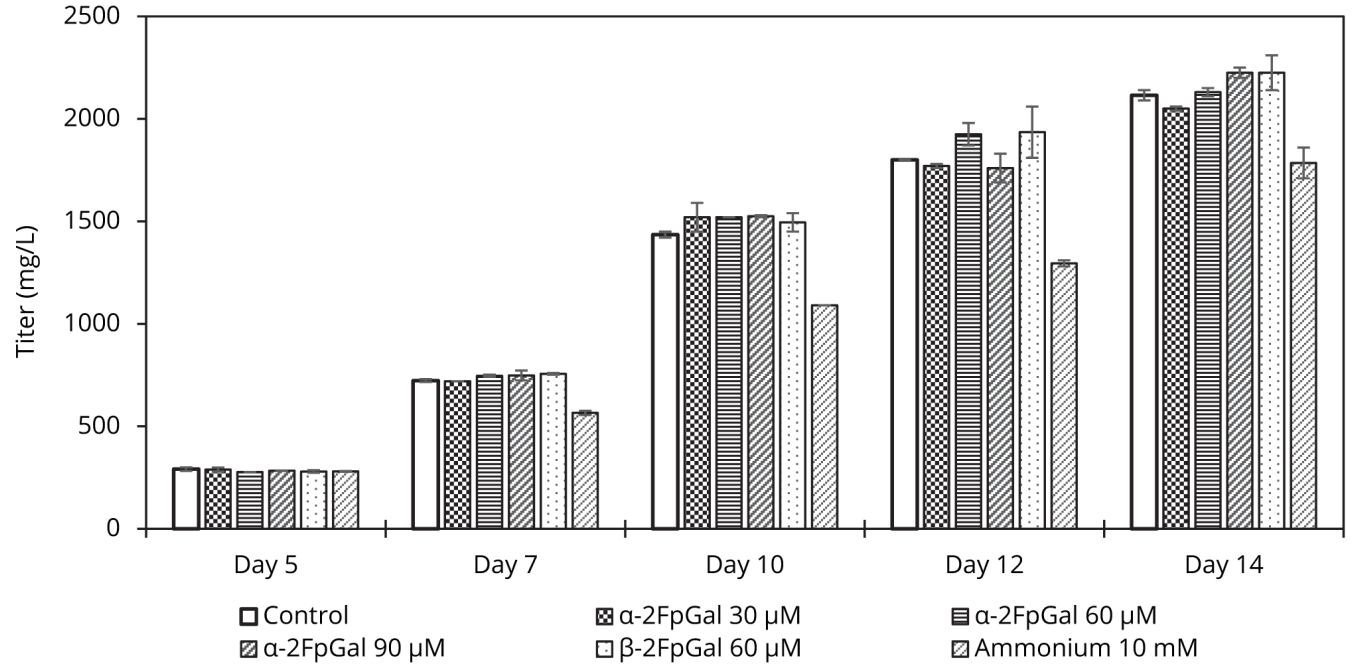

Figure 5.5 - (A) Viable cell densities of cell line A cultures supplemented with 0-90 $\mu \mathrm{M} \alpha$ 2F-peracetyl-galactose, $60 \mu \mathrm{M} \beta$-2F-peracetyl-galactose, or $10 \mathrm{mM}$ ammonium in ST. (B) Viabilities. (C) Protein titer for each concentration on culture days 5, 7, 10, 12 and 14. Each condition was conducted in duplicates. All points are mean values of the corresponding conditions and the error bars report the maximum and minimum values. 
A

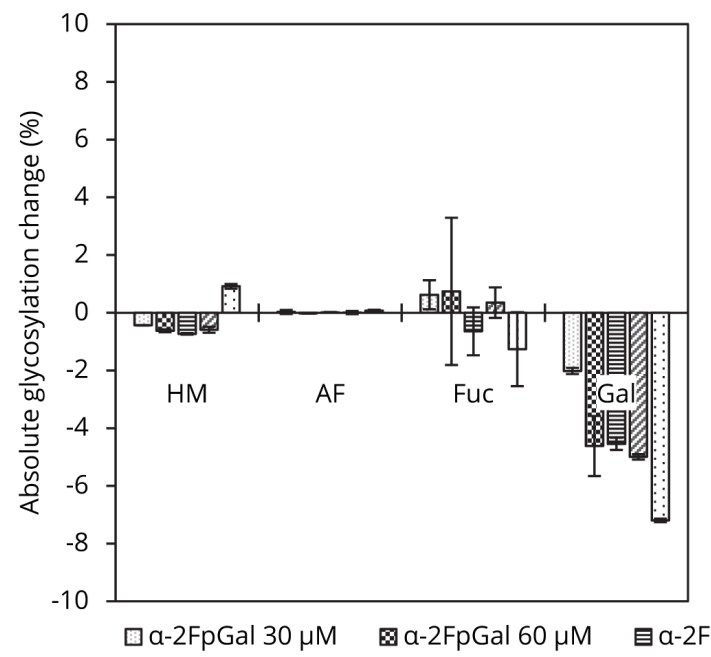

B

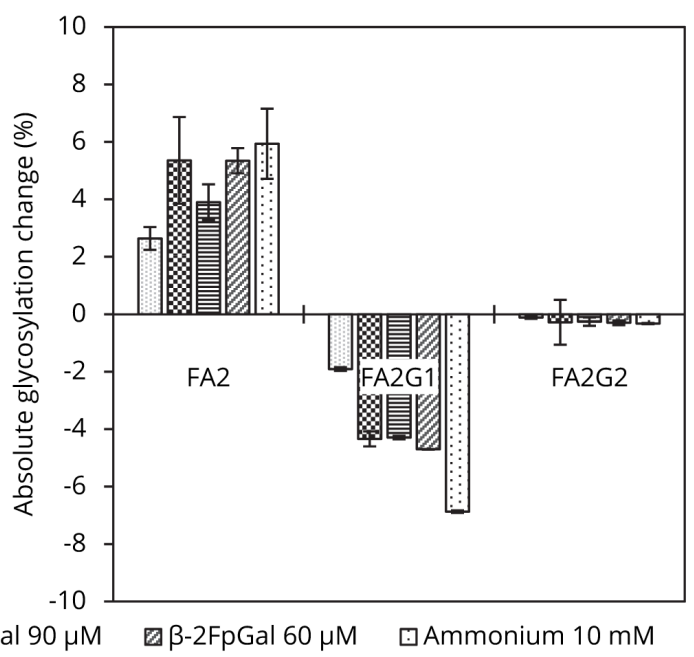

Figure 5.6 - (A) Absolute change of the overall glycosylation pattern compared to the control in function of the $\alpha$ - and $\beta$-2F-p-galactose concentration in medium in comparison with $10 \mathrm{mM}$ ammonium in cell line A cultures. (B) Absolute change of galactosylation compared to the control in function of the $\alpha$ - and $\beta$-2F-p-galactose concentration in medium in comparison with $10 \mathrm{mM}$ ammonium. Each condition was conducted in duplicates. All bars represent mean values of the corresponding conditions analyzed by CGE-LIF and the error bars report the maximum and minimum values. 
yielded $3200 \mathrm{mg} / \mathrm{L}$ (control: $3300 \mathrm{mg} / \mathrm{L}$ ).

A

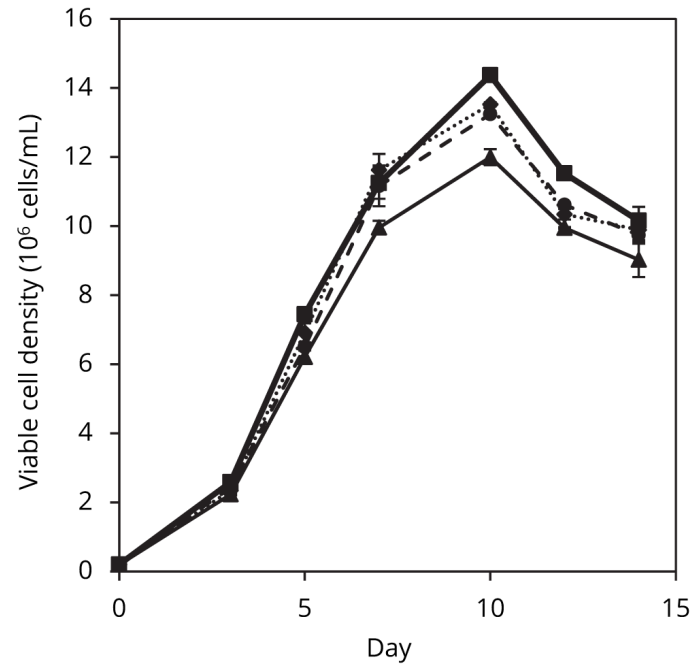

$\longrightarrow$ Control $\quad \ldots \bullet \cdots$ a-2FpGal $30 \mu \mathrm{M}$
B

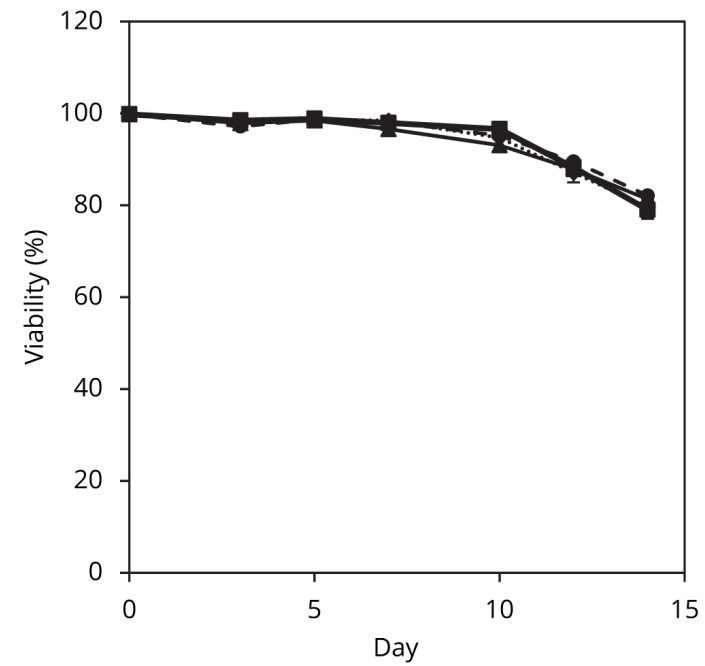

C

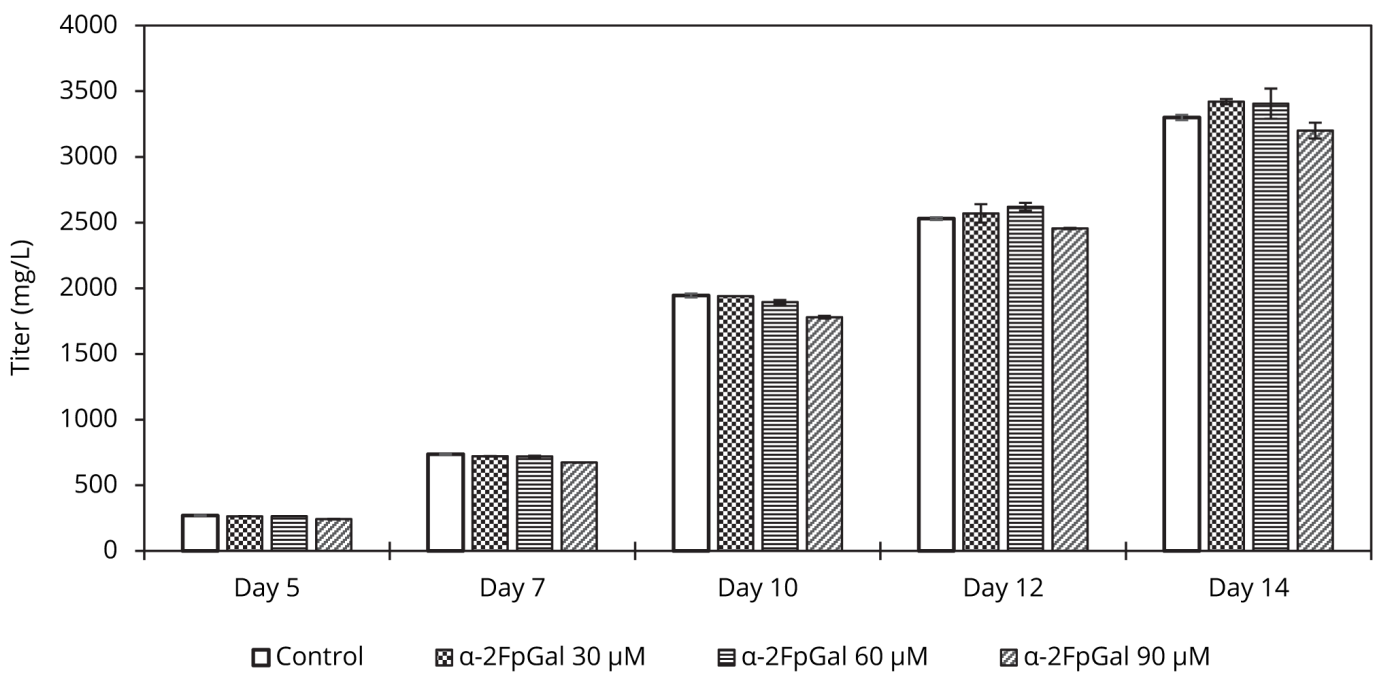

Figure 5.7 - (A) Viable cell densities of cell line B cultures supplemented with 0-90 $\mu \mathrm{M} \alpha-2 \mathrm{~F}$ peracetyl-galactose in ST. (B) Viabilities. (C) Protein titer for each concentration on culture days 5, 7, 10, 12 and 14. Each condition was conducted in duplicates. All points are mean values of the corresponding conditions and the error bars report the maximum and minimum values.

Figure 5.8A shows the absolute glycan change in function of the $\alpha$-2F-peracetyl-galactose concentration in the medium. Like for cell line A, the galactose analog reduced galactosylation. The reduction amounted to $-0.7,-1.6$ and $-1.1 \%$ at respectively 30,60 and $90 \mu \mathrm{M}$. Its presence 
also had a non negligible effect on the overall fucosylated species $(+0.9 \%)$. Figure $5.8 \mathrm{~B}$ zooming in the individual terminal galactose species displays a $-0.6,-1.3$ and $-0.9 \%$ decrease for FA2G1 and small change of the FA2G2 abundance of $-0.09,-0.19$ and $-0.19 \%$ at respectively 30 , 60 and $90 \mu \mathrm{M}$. One should keep in mind that 2AB-UPLC rather than CGE-LIF was used to quantity the glycan pattern of cell line B cultures.

A

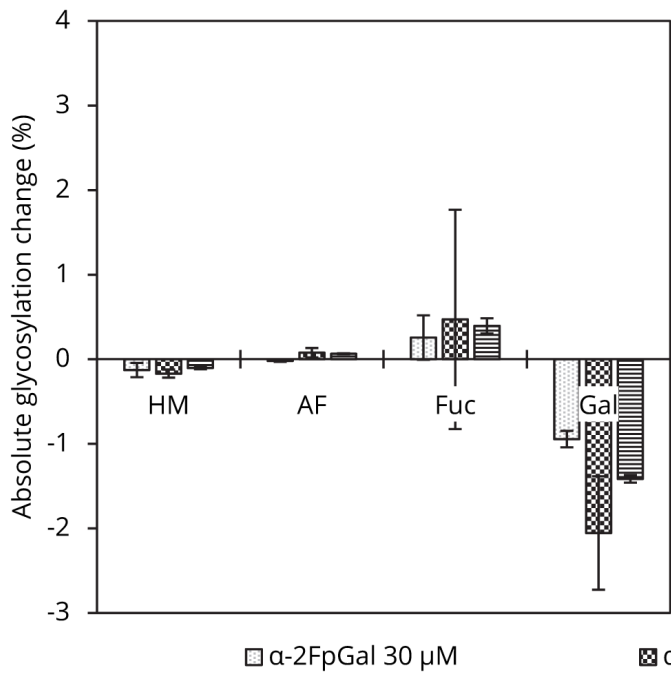

B

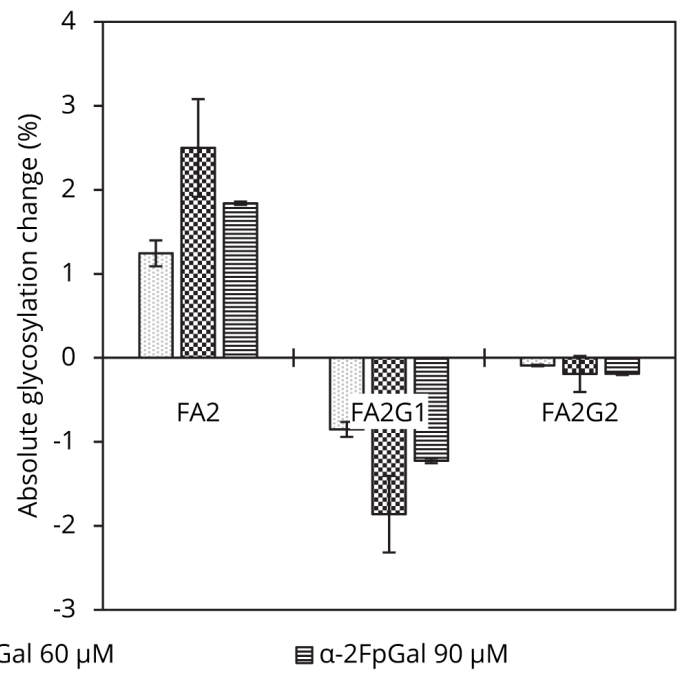

Figure 5.8 - (A) Absolute change of the overall glycosylation pattern compared to the control in function of the $\alpha$-2F-p-galactose concentration in medium of cell line B cultures. (B) Absolute change of galactosylation compared to the control in function of the $\alpha$-2F-p-galactose concentration in medium of cell line B cultures. Each condition was conducted in duplicates and analyzed by $2 \mathrm{AB}-U P L C$. All bars represent mean values of the corresponding conditions and the error bars report the maximum and minimum values.

Rather than supplementing the medium prior to inoculation, it was decided to start the galactose addition on day 3,5 or 7 . Figure 5.10A shows how $\beta$-2F-p-galactose affected the viable cell density in function of the addition time. The peak cell densities were reached on day 7. No significant difference between the control and the on day 3 supplemented cultures was observed. They both levelled off at 20.8 and $21.2 \times 10^{6}$ viable cells $/ \mathrm{mL}$, respectively. The feed on day 5 reduced the maximum cell density, reaching $18.6 \times 10^{6}$ viable cells $/ \mathrm{mL}$. Overall, it can be assumed that the presence of $\beta$-2F-p-galactose induced limited changes on cell growth until day 7. In the second half of the culture, both the cell density and the viability (5.10B) are correlated with the feed timing. Supplementation on day 3 most strongly impacted the course of the culture. At harvest, the cell density amounted to $9.8 \times 10^{6}$ viable cells $/ \mathrm{mL}$ (control: $11.8 \times$ $10^{6}$ viable cells $/ \mathrm{mL}$ ). The viability dropped notably faster than in the control, falling below $60 \%$. Feed addition on day 5 and 7, respectively, entailed viabilities on day 14 of 78 and $90 \%$ (control: 93\%). According to figure 5.10C no clear titer trend came forward. Early supplementation induced a slight titer increase on days 7 and 10, but then reduced productivity at later stages of the culture. While the control yielded $2870 \mathrm{mg} / \mathrm{L}$, supplementation on day 3, $2560 \mathrm{mg} / \mathrm{L}$. 
The addition of $\beta$-2F-p-galactose on day 5 favored antibody expression, producing $3215 \mathrm{mg} / \mathrm{L}$. Addition on day 7 , resulted in a considerably lower protein titer: $2100 \mathrm{mg} / \mathrm{L}$. In comparison to media supplementation, introducing $\beta$-2F-p-galactose by means of feeding, limits detrimental effects on the cell performance. Even when starting the feed on day 7, the final additive concentration in the supernatant was considerably higher than in media supplementation, where important growth and productivity reduction resulted as mentioned previously.

Feed optimization, using $\beta$-2F-p-galactose resulted in an important inhibition of galactosylated glycoforms as shown in figure $5.10 .11 .5 \%$ of the secreted antibodies in the control were galactosylated. Feed addition from day 3 resulted in the strongest inhibition: overall galactosylation decreased by $-8.5 \%$. Conditions starting the feed on day 5 and 7 brought about reductions of -7.0 and $-4.6 \%$, respectively. Like in the medium supplementation experiments, $\beta$-2F-p-galactose specifically target galactosylation. Effects on the other glycan species remained small. The mono-galactosylated species dropped by $-8.0,-6.7$ and $-4.4 \%$, while the di-galactosylated entity decreased by $-0.41,-0.33$ and -0.19 when starting the feed on day 3,5 and 7 , respectively (figure 5.10). The amplitude of the galactosylation inhibtion was correlated with the start date of the supplement feed, and thus, the level of $\beta$-2F-p-galactose in the supernatant.

\subsubsection{Spermine}

In the frame of an exploratory screening exercise using cell line A in 96-DWP a correlation between spermine supplementation and galactosylation inhibition was observed. In figure 5.11A one can note that spermine supplementation of 1 to $200 \mu \mathrm{M}$ favored cell growth. The control cultures reached the maximum viable cell density of $8.2 \times 10^{6}$ cells $/ \mathrm{mL}$. At spermine concentrations between 1 and $20 \mu \mathrm{M}$ the peak moved to day 7 and further increased with greater supplement concentrations, reaching a maximum value of $18.6 \times 10^{6} \mathrm{VC} / \mathrm{mL}$. High concentrations ( $\geq 50 \mu \mathrm{M}$ ) reduced growth once again. According to figure $5.11 \mathrm{~B}$, spermine increased the cell viability. Both on day 10 and 14, the productivity became more abundant between 1 and $20 \mu \mathrm{M}$ (figures 5.11C and D). Beyond, the antibody production tapered. On average, the control cultures yielded $620 \mathrm{mg} / \mathrm{L}$, and at a concentration of $20 \mu \mathrm{M}$ spermine, it ascended to $1669 \mathrm{mg} / \mathrm{L}$.

Spermine already produced a strong galactosylation inhibition at the lowest concentration (figure 5.12A). At $1 \mu \mathrm{M}$, a reduction of $-3.1 \%$ resulted and progressively increased to $-3.4,-4.7$, $-5.0,-5.9$, and $-7.0 \%$ at respectively $5,10,20,50$, and $100 \mu \mathrm{M}$. At $200 \mu \mathrm{M}$ the galactosylation level declined by $-5.7 \%$. Spermine addition exhibited a limited effect on high mannose, afucosylated as well as fucosylated species. The predominant galactosylated species (FA2G1) mainly contributed to the observed effect (figure 5.12B). It decreased by $-2.9,-3.0,-4.3,-4.6$, $-5.4,-6.5$, and $-5.3 \%$ at $1,5,10,20,50,100$, and $200 \mu \mathrm{M}$, respectively. The digalactosylated glycan varied to a lower extent. The decrease with respect to the control amounted -0.2 to $-0.5 \%$. 
A

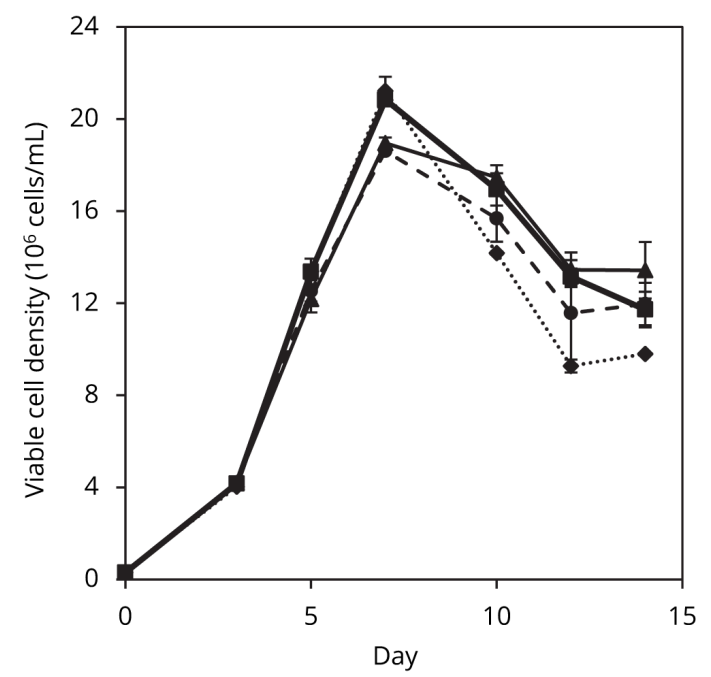

B

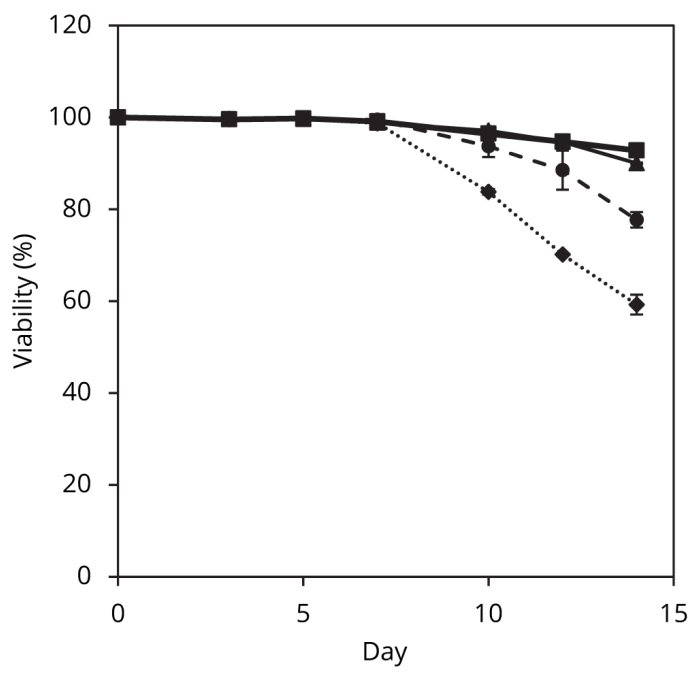

$\longrightarrow$ Control $\cdots \cdots \cdots \beta-2 F p G a l$; feed day 3

ß-2FpGal; feed day 5

$\longrightarrow$ - $-2 F p G a l ;$ feed day 7

C

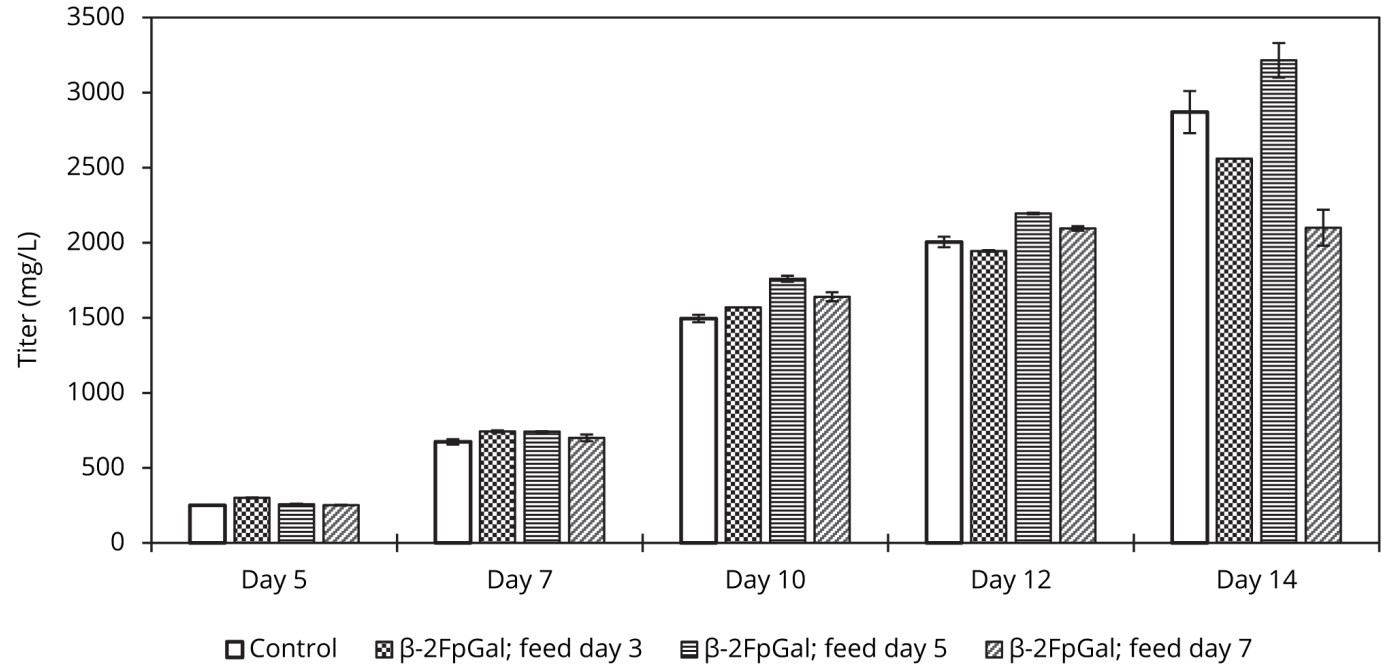

Figure 5.9 - (A) Viable cell densities of cell line A cultures in function of the feed timing of $\beta$-2F-p-galactose in ST. (B) Viabilities. (C) Protein titer for each condition on culture days 5, 7 , 10, 12 and 14. Experiments were conducted in duplicates. All points are mean values of the corresponding conditions and the error bars report the maximum and minimum values. 
A

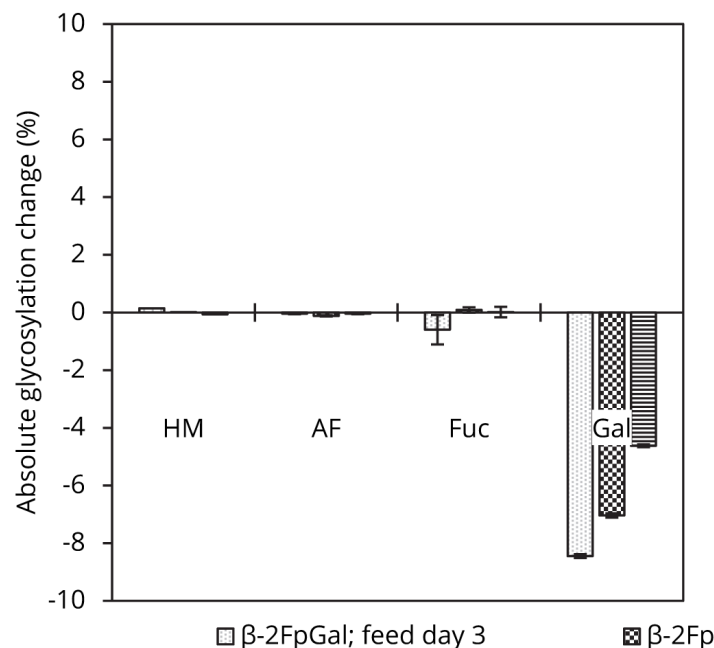

B

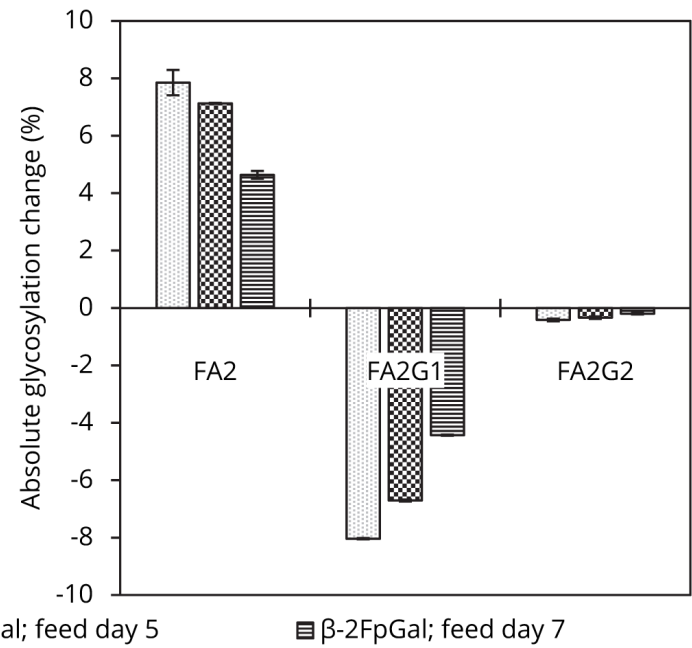

Figure 5.10 - (A) Absolute change of the overall glycosylation pattern compared to the control in function of the feed timing of $\beta$-2F-p-galactose in cell line A cultures. (B) Absolute change of galactosylation compared to the control in function of the feed timing of $\beta$-2F-p-galactose in cell line A cultures. Experiments were conducted in duplicates and supernatant analyzed by CGE-LIF. All bars represent mean values of the corresponding conditions and the error bars report the maximum and minimum values.

\subsubsection{L-ornithine}

According to figure 5.13, L-ornithine addition in the range of 0.5 to $15 \mathrm{mM}$ had no significant influence on the cell culture performance of cell line A. Peak cell density was reached on day 5 or 7 . Maximum values were in the interval of 7.6 to $10.8 \times 10^{6} \mathrm{VC} / \mathrm{mL}$. The mean viabilities of the control decreased faster than the supplemented conditions. Nonetheless, due to the high inter-replicate variability, the behavior of the cultures were likely similar. Despite the important variability, one can notice that L-ornithine may have effected a small titer increase at the end of the culture (figure 5.13D).

In the tested L-ornithine concentration range, the overall glycosylation levels decreased in the range between -1.9 and $-4.4 \%$ (figure $5.14 \mathrm{~A}$ ). Contrary to $2 \mathrm{~F}$-p-galactose and spermine, increasing concentrations did not bring about stronger galactosylation effects. Already at $0.5 \mathrm{mM}$ L-ornithine in the medium galactosylation decreased by $-4.2 \%$. High mannose, afucosylated and fucosylated species did not vary significantly. According to figure 5.14B, L-ornithine reduced mono-galactosylated forms (FA2G1) by -1.8 to $-3.9 \%$. The inter-replicate variability of the di-galactosylated form was in general greater than the mean reduction. 


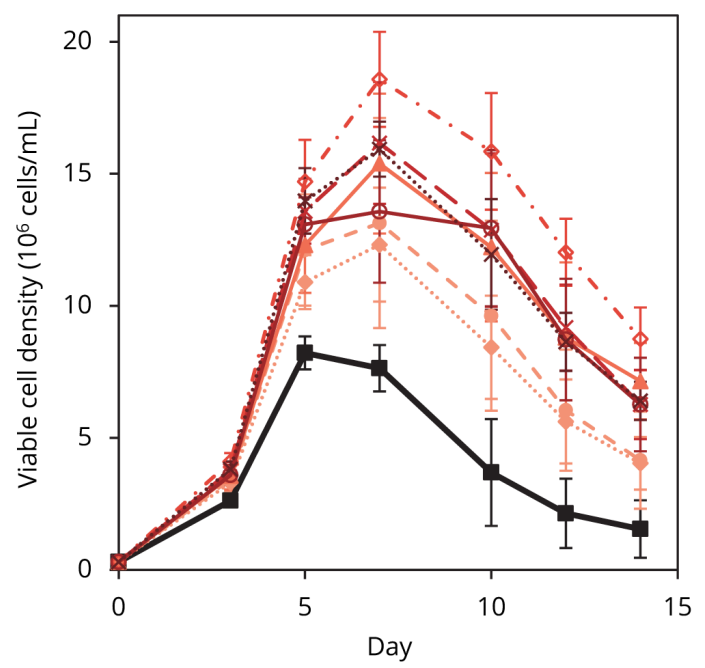

B

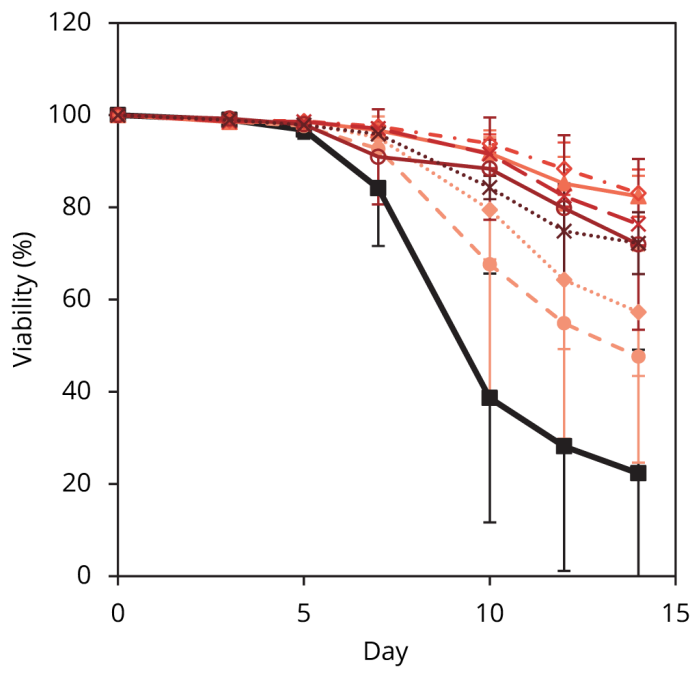

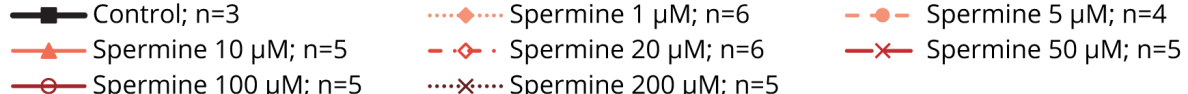

C

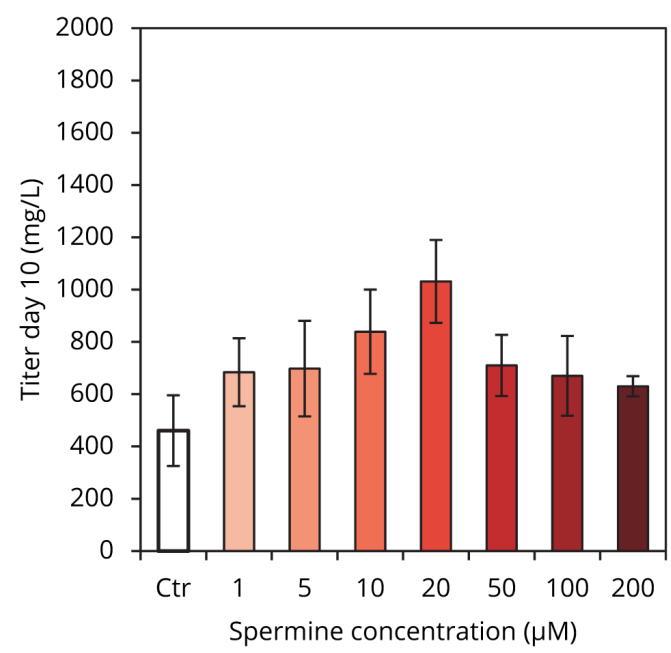

$\mathrm{D}$

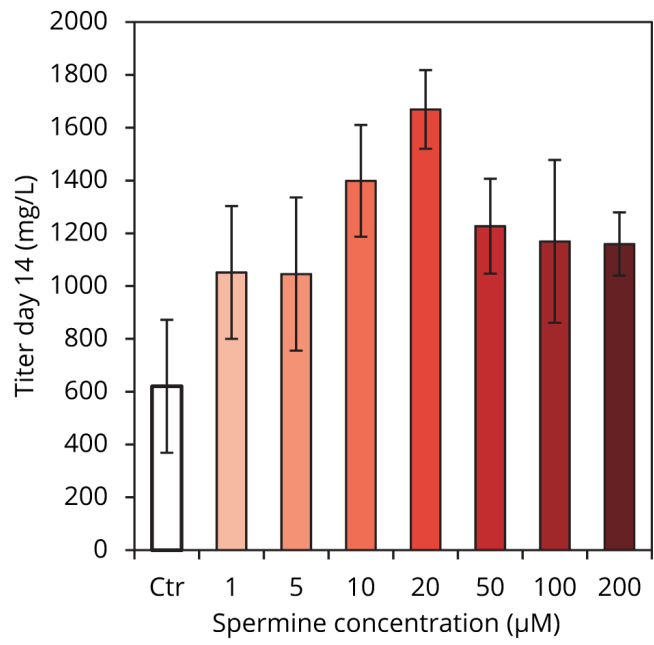

Figure 5.11 - (A) Viable cell densities of cell line A cultures supplemented with $0-200 \mu \mathrm{M}$ of spermine. (B) Viabilities. (C) Product titers on day 10. (D) The titer in the harvest on day 14. The number of replicates of each condition is indicated in chart A. All points are mean values of the corresponding replicates and the error bars report the standard deviation of the replicates. 
A

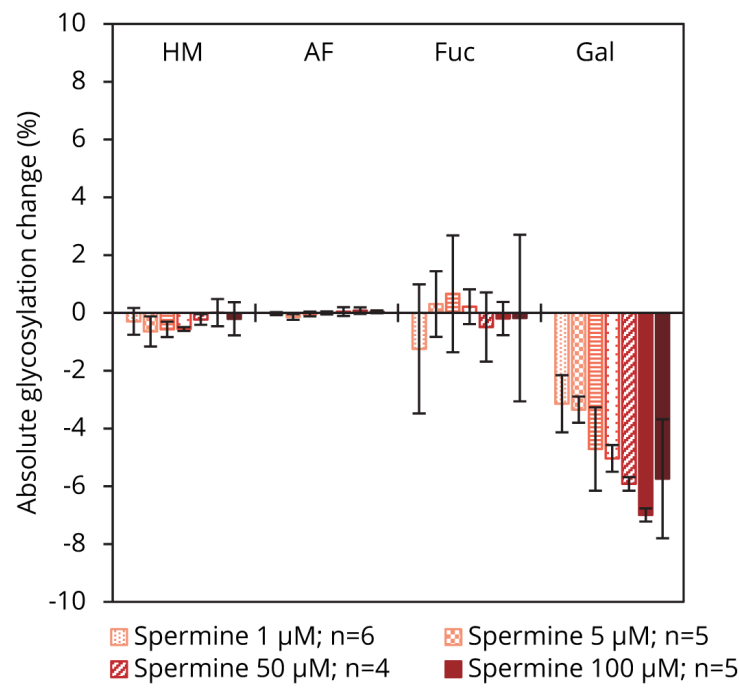

B

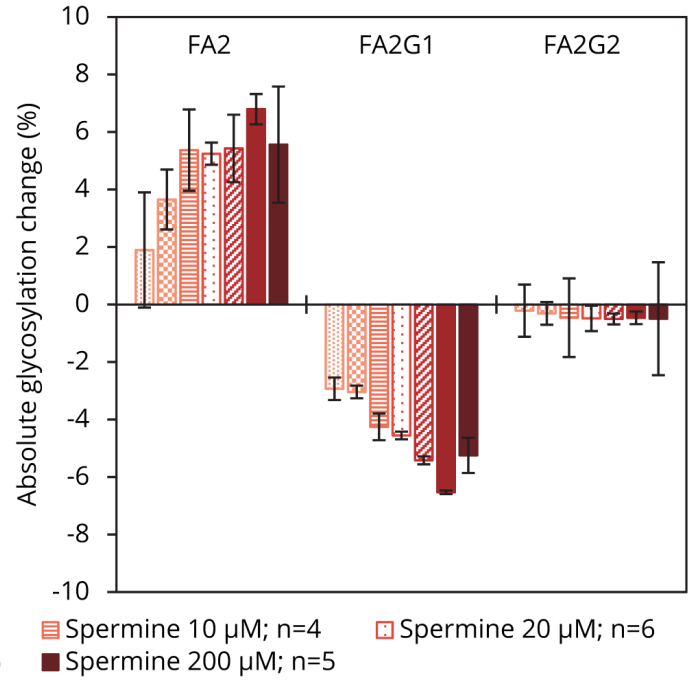

Figure 5.12 - (A) Absolute change of the overall glycosylation pattern compared to the control in function of the spermine concentration in the medium. (B) Absolute change of galactosylation compared to the control in function of the spermine concentration in the medium. All points are mean values of the corresponding replicates analyzed by CGE-LIF and the error bars report the standard deviation of the replicates.

\subsection{Discussion}

2F-peracetyl-galactose consistently and reproducibly diminished the level of galactosylation in 96-DWP and ST in two different cell lines. The performance of the $\alpha$-and $\beta$-anomers was comparable in cell line A. A maximal reduction of $-5 \%$ resulted in the presence of $60 \mu \mathrm{M}$ $\beta$-2F-peracetyl-galactose in ST with comparable cell culture performance. Stronger inhibition effects were observed at higher concentrations in 96-DWP at the expense of growth and titer reductions. The glycosylation machinery of cell line A responded more strongly to the presence of $\alpha$-2F-peracetyl-galactose than cell line B. The galactose analog at $60 \mu \mathrm{M}$ reduced galactosylation by $-4.6 \%$ in cell line A versus $-1.6 \%$ in cell line B. This highlights that the metabolism of each cell line may react in a different manner to various medium compositions, and hence environmental changes. Feed optimization proofed to be an excellent strategy to further enhance the effect of the supplement. While media supplementation with $\beta-2 \mathrm{~F}$ peracetyl-galactose in cell line A induced a maximum reduction of the overall galactosylation level of $-5.0 \%$, feed supplementation yielded a $-8.0 \%$ decrease, which corresponds to a $60 \%$ stronger effect. Furthermore, feed supplementation allowed to increase the total amount of the additive, entailing considerably smaller detrimental effects on cell culture performance in comparison with medium supplementation. Possibly 2F-peracetyl-galactose specifically inhibits galactosyltransferase as 2F-peracetyl-fucose and 3F-neuramic acid do their corresponding enzyme as described in the literature ${ }^{112}$. To confirm this hypothesis, more specific enzymatic assays are required. Spermine addition to the medium resulted in a comparable 


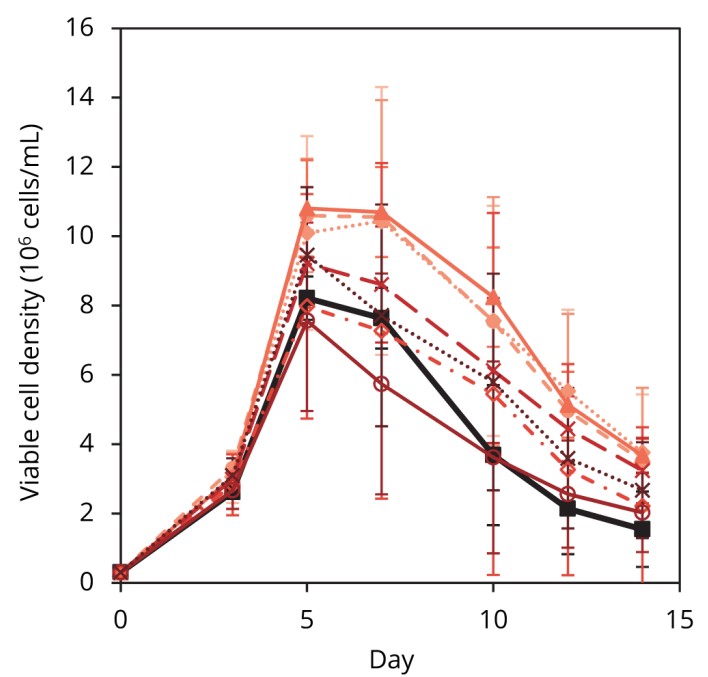

B

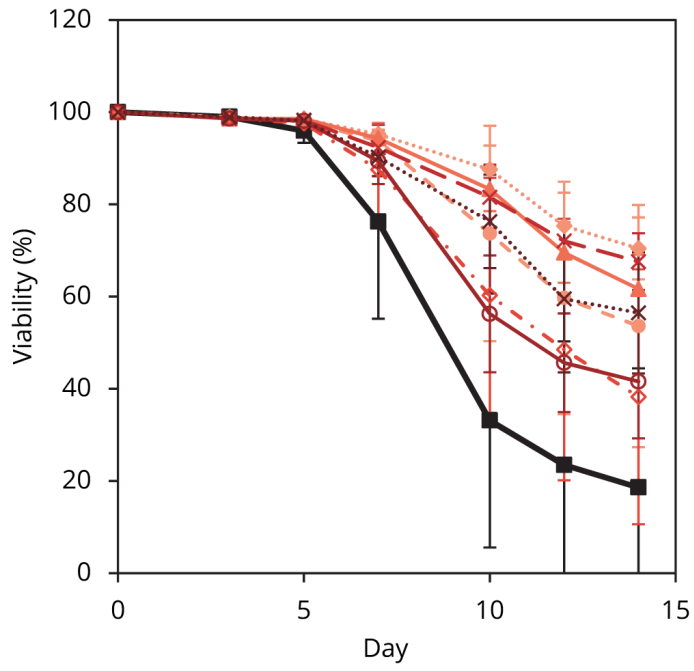

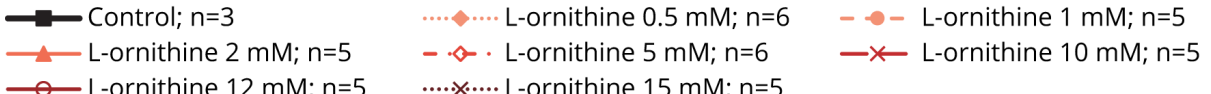

C

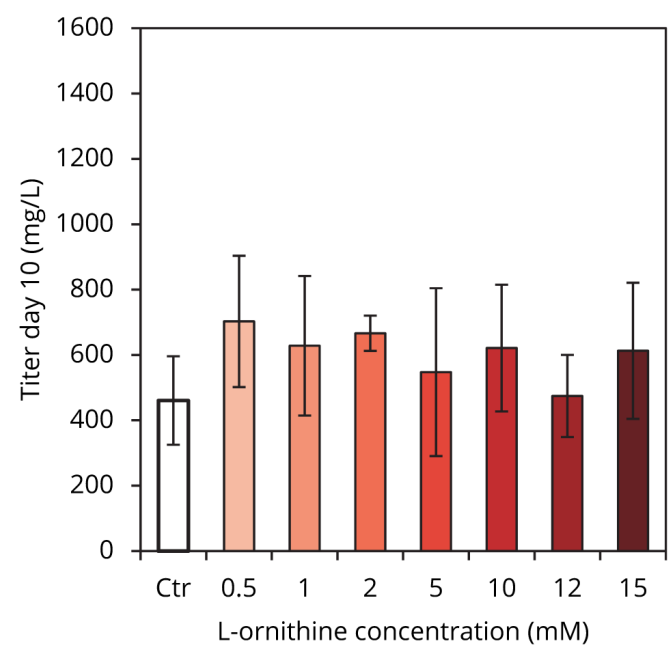

D

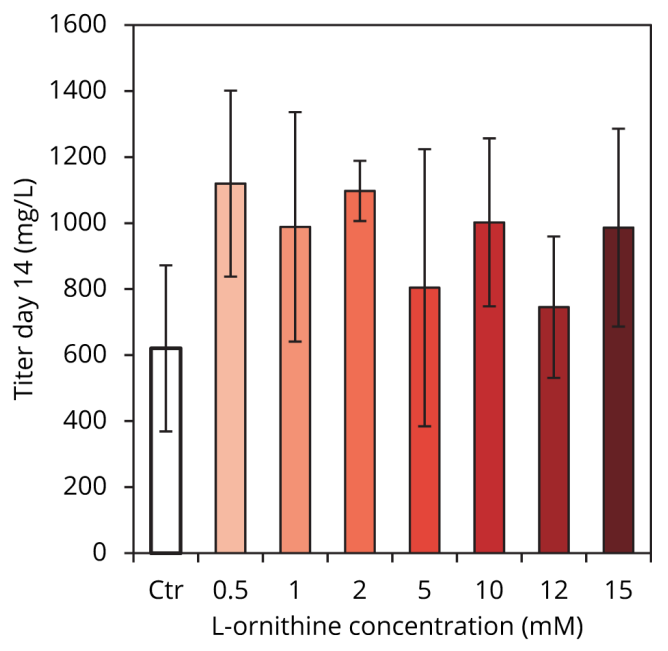

Figure 5.13 - (A) Viable cell densities of cell line A cultures supplemented with 0-15 mM of L-ornithine. (B) Viabilities. (C) Product titers on day 10. (D) The titer in the harvest on day 14. The number of replicates of each condition is indicated in chart A. All points are mean values of the corresponding replicates and the error bars report the standard deviation of the replicates. 
A

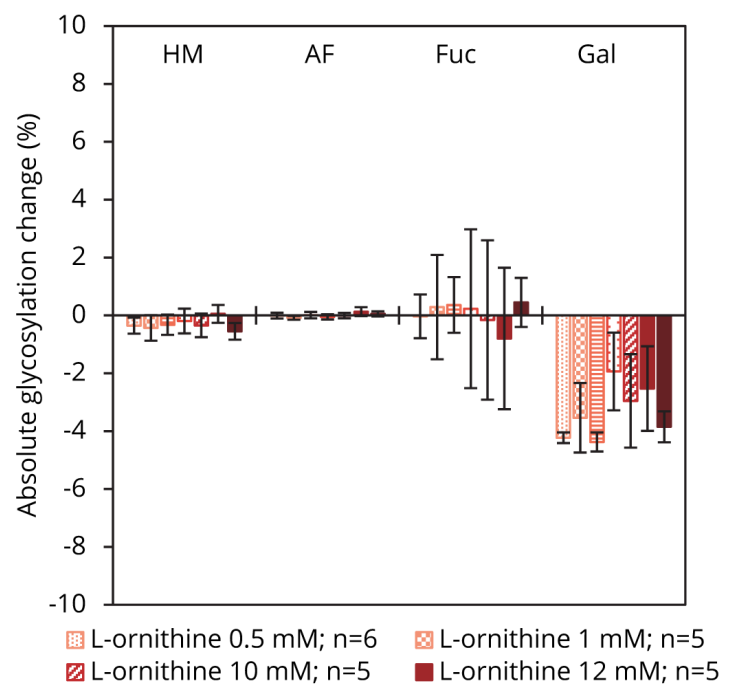

B

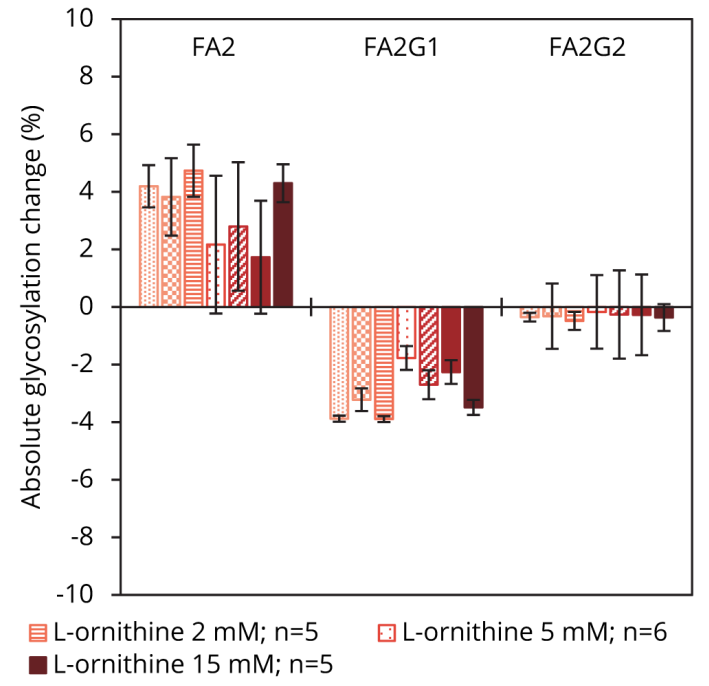

Figure 5.14 - (A) Absolute change of the overall glycosylation pattern compared to the control in function of the L-ornithine concentration in the medium. (B) Absolute change of galactosylation compared to the control in function of the L-ornithine concentration in the medium. All points are mean values of the corresponding replicates analyzed by CGE-LIF and the error bars report the standard deviation of the replicates.

reduction of galactosylation levels in 96-DWP. These findings need to be confirmed in ST. On the other hand, L-ornithine did reduce galactosylation, but no proportionality with the supplement concentration was observed. The absence of trend may be explained by the fact that the L-ornithine concentration range was too high and the maximum inhibition level had already been reached. It would be worthwhile to test lower concentration ranges in spin tubes to validate this hypothesis and to confirm the observed inhibition effects in 96-DWP. The results show that both medium and feed supplementation of galactose analogs, a polyamine and a non proteinogenic amino acid consistently and specifically reduced the level of galactosylation in fed-batch cultures. A report described the role of spermine in the maturation of intestinal galactosylation in rat intestine, showing that the amount of digested polyamines correlated with increased galactosyltransferase activity ${ }^{239}$. Interestingly, in the same study spermine had no influence on the galactosyltransferase activity in vitro. They concluded that the maturation of intestinal galactosylation may well be a multifactoral event in which spermindine and spermine are implicated. They further observed that spermine substitution by L-ornithine, a polyamine precursor, resulted in similar galactosyltransferase activity in vivo, and like in the case of spermine, L-ornithine did not directly affect the enzyme activity in vitro.

In the experimental conditions of the present study, galactosylation decreased with increasing levels of spermine. For the time being, the results show that spermine plays a role in glycosylation, however mechanistic studies will be required to understand how spermine affects the glycosylation pathway in CHO-cells. Moreover, spermine supplementation entailed a consid- 
erable increase of the cell density. The peak cell density doubled at concentration of $20 \mu \mathrm{M}$. The literature has reported a link between spermine and growth. Depletion of spermine and spermidine led to an entire arrest in translation and growth in HEK293 cells, and a direct role for polyamines in the initiation of translation was suggested ${ }^{240}$. Other scientists observed that polyamines were needed at the initiation and at the elongation steps of translation: they showed that spermine is a precursor in the hypusination of the eukaryotic initiation factor $5 \mathrm{~A}$ (eIF5A) that acts as a translation elongation factor ${ }^{241}$. These findings are congruent with the increased cell proliferation in the experimental conditions of the present study.

The results of this work show that the presented approach can be successfully used to identify potential galactosylation modulators. The screening of compounds in a high-throughput device such as 96-DWP allowed to identify potent compounds, to determine optimal concentration ranges and to conduct a preliminary assessment of potential detrimental effects on growth and productivity. The trends observed in 96-DWP were confirmed in the more robust ST scale following the example of previous work ${ }^{28}$. Hence, the larger variability of 96DWP was not an obstacle. No major differences were observed for $\alpha$-2F-peracetyl-galactose, using either CGE-LIF or 2AB-UPLC for glycan analysis. CGE-LIF provided a good peak resolution for galactosylation and the co-elution of various high mannose species was not a concern. Due to its much higher throughput, CGE-LIF is the assay of choice for screening of galactosylation-modulating compounds.

\subsection{Conclusion}

Changes of the environment in which mammalian cells were cultured in induced modification of the cell metabolism, resulting in an altered galactosylation pattern. Medium and feed supplementation with galactose analogs ( $\alpha$ - and $\beta$-peracetyl-galactose), spermine and L-ornithine specifically reduced the level of galactosylation of mAbs. Media supplementation worked well, nonetheless, feed supplementation further increased galactosylation inhibition, while limiting detrimental effects on cell culture performance. This targeted metabolic approach proofed to produce consistent and reproducible changes of the pathway involved in the attachement of terminal galactose onto the existing $\mathrm{N}$-glycan moeity of mAbs. The results demonstrate that high-throughput fed-batch cultures in 96-DWP allow to identify potent compounds and to define optimal concentration ranges prior to scaling up to the more robust shake tube scale. Galactosylation modulation trends observed in 96-DWP were confirmed in shake tubes. 


\section{Chapter 6}

\section{Identification of Compounds Influencing Low-Molecular-Weight Species}

\subsection{Introduction}

Therapeutic biomolecules have revolutionized the treatment of patients ${ }^{242}$. Contrary to the proteins of animal origin, recombinant analogs of insulin from E.coli cultures and human growth hormone produced in mammalian cells have provided the patients with access to safer and more efficacious drug products from the advent of biotechnology until today. They enabled purity improvements of insulin preparations, for instance, and patients treated with this biotherapeutic displayed lower prevalence and titers of anti-insulin antibodies ${ }^{3}$. While recombinant drugs are in general well tolerated and their advantages largely outweigh the downsides, their safety and efficacy can be severely impaired by the development of immunogenic reactions against the therapeutic protein ${ }^{243}$. Many parameters affect protein immunogenicity, including structural features (sequence variation and glycosylation), storage conditions (denaturation, or aggregation caused by oxidation), contaminants or impurities in the preparation, dose and length of treatment, as well as the route of administration, appropriate formulation and the genetic characteristics of patients ${ }^{13}$. Among some glycoforms, high mannose and galactose-1,3- $\alpha$-galactose ( $\alpha$-gal) are highly immunogenic and in most cases should be limited during manufacturing ${ }^{205}$. Immunogenicity may lead to many clinical consequences, such as altered pharmacokinetics of the proteins and inhibition of the therapeutic effect or the neutralization of essential endogenous proteins ${ }^{244}$. A report described the capacity of the immune system to produce specific antibody responses due to immunogens of differing molecular weight and size and identified a threshold for immunogenicity of $10 \mathrm{kDa}^{245}$. Intense research efforts have been dedicated to predict and reduce immunogenicity ${ }^{246}$. In line with the huge 
success of monoclonal antibodies since the late 1990's ${ }^{247}$, the administered doses have massively multiplied ${ }^{248}$. On the other hand, in a study with twenty-eight single clonal CHO cell lines, correlations were identified between the antibody titer and the level of both aggregation and low-molecular species ${ }^{249}$. They concluded that correct and efficient antibody assembling and/or folding are indispensable for high titer and low aggregates contents. Protein aggregation is a common issue for quality, safety, and efficacy of antibodies ${ }^{168}$. These also called high-molecular-weight species (HMW) have to be limited to avoid immunogenic reactions in the injected patients. It is also important to keep in mind that the productivity of recombinant cell culture processes has substantially increased from $50 \mathrm{mg} / \mathrm{L}$ in batch to titers of 10 to $13 \mathrm{~g} / \mathrm{L}$ nowadays in fed-batch culture of 2 to 3 weeks ${ }^{33,34}$. Moreover, the literature describes how cell culture conditions can be identified to control protein aggregation in $\mathrm{CHO}$ cell cultures ${ }^{54}$. A variety of chromatographic resins have been designed for efficient aggregate removal. A selection of these resins were included in the proprietary purification platform process. It efficiently reduces the level of aggregation throughout the chromatography steps, enabling to reach low levels thereof. For this reason, this chapter solely focuses on low-molecular-weight species. Their removal in the purification proves to be a greater challenge and arises the need to adjust cell culture conditions to reduce cleavage and/or protein expression errors. A variety of adverse effects stems from fragmentation, including reduced biological activity, shorter half-life and immunogenicity reactions ${ }^{68}$.

Literature seems to agree upon the fact that mammalian cell culture may entail formation of low-molecular-weight species. Only a few article address the origin thereof and provide specific mechanistic insight for the context of cell culture processes. The quality of the folding process plays an important role on the abundance of LWM species ${ }^{249}$. On the other hand, heavy and light chains are connected to each other via disulfide bridges ${ }^{250}$. Both the disruption of these bonds and the cleavage of a covalent peptide bond by coexistent spontaneous and enzymatic reactions have been evoked to be at the core of LMW formation ${ }^{68}$. The sites in the flexible hinge region of mAbs within the constant heavy 1 and 2 domains ( $\mathrm{CH} 1$ and $\mathrm{CH} 2$ ) and around domain interfaces are most prone to fragmentation ${ }^{179,187}$. Known for its ability to provide oxidizing potential in redox reactions, copper plays a pivotal role in non-enzymatic cleavage ${ }^{185}$. Increasing concentration of cupric ions in solution resulted in greater fragmentation rates and inhibited by chelating agents ${ }^{179}$. Moreover, three enzymatic mechanisms including the thioredoxin system (TrxR), glucose-6-phosphate dehydrogenase (G6PD), and hexokinase have been identified to be responsible for disulfide bond reduction ${ }^{185,186}$.

The proprietary mammalian cell culture platform process has been optimized to deliver consistently high titers, using fed-batch mode. This is achieved by maintaining high cell viabilities $(\geq 80 \%)$ until the end of the culture. A relatively small percentage of the expressed antibodies aggregate and are cleaved. However, due to the poor fragment removal in the purification process, they have to be limited in cell culture. This chapter presents potential compounds and mechanisms influencing the production of low-molecular-species. High-throughput media blending approaches ${ }^{26,27,251}$ and mechanistic studies were called upon based on the information provided by the literature and hypotheses resulting from the experimental results. Due to 
the relatively few number of scientific reports that address LMW formation in mammalian cell culture, it was decided to perform a general reshuffling of the platform medium components to identify potential levers for LMW content reduction. In-house development data provides evidence that the medium composition plays an important role in the underlying mechanisms: when the proprietary medium was replaced by a commercial medium the LMW level was significantly lower in some cases. Subsequently, the predominant LMW species were identified by in-depth analysis of the chromatograms. Thus, potential cleavage mechanisms were proposed based on the nature of the fragment species in the proprietary cell culture process.

Despite the exploratory nature of the study, the results show that a combination of media blending of fed-batch cultures in 96-deepwell plates with no prior specific mechanistic knowledge related to the proprietary platform process allowed to observe trends and provide evidence that the hypotheses may be confirmed in future confirmation experiments. The data also highlight the fact that this early research needs to be further advanced until final conclusions can be drawn. Nonetheless, high-throughput screening proved to be, once again, a powerful method to search for the needle in the haystack. The identified levers in 96-DWP oriented the search target, thus narrowing down the scope of the subsequent testing of additional compounds and mechanistic studies.

\subsection{Materials and Methods}

\subsubsection{Inoculum Preparation}

Two recombinant cell lines were used in the frame of this study. A CHO-S derived clonal cell line expressing a human monoclonal IgG1 antibody (cell line A) and a CHO-K1 derived clonal cell line expressing a humanized monoclonal IgGl antibody (cell line B). Cells were first expanded in shake tubes or shake bottles in proprietary medium containing methionine sulfoximine (MSX) in multiple passages every 2-3 days, diluting to 0.2 or $0.3 \times 10^{6}$ viable cells $/ \mathrm{mL}$ for at least 14 days. The tubes were maintained in a shaker incubator at $36.5{ }^{\circ} \mathrm{C}, 5 \%$ $\mathrm{CO}_{2}, 80 \%$ humidity and $320 \mathrm{rpm}$ agitation (ISF1-X, Adolf Kühner, Birsfelden, Switzerland or Multitron Cell, Infors HT, Bottmingen, Switzerland).

\subsubsection{Cell Culture Conditions}

The high-throughput fed-batch cell culture was performed on a robotic liquid handling platform (Biomek FX, Beckman Coulter, Brea, CA). Exponentially growing CHO-S cells were seeded into a shaking 96-DWP filled with proprietary medium enriched with amino acids in the absence of MSX at a viable cell density of $0.30 \times 10^{6}$ viable cells $/ \mathrm{mL}$ and exponentially growing $\mathrm{CHO}-\mathrm{K} 1$ cells at $0.20 \times 10^{6}$ viable cells $/ \mathrm{mL}$. Table 6.1 shows the concentration ranges of 
the amino acid concentration increases in the cell culture medium before seeding. The plates were incubated with vented lids to minimize evaporation in a shaker incubator at $36.5^{\circ} \mathrm{C}$, $5 \% \mathrm{CO}_{2}, 90 \%$ humidity and $320 \mathrm{rpm}$ agitation (ISF1-X, Adolf Kühner, Birsfelden, Switzerland) for 14 days. $400 \mathrm{~g} / \mathrm{L}$ glucose solution, chemically-defined feed (CD-feed) containing over 30 components and alkaline amino acid solution were added on day 3, 5, 7, 10 and 12. Prior to each feeding and at the end of the culture on day 14 , samples $(\leq 40 \mu \mathrm{L})$ were drawn for growth and viability assessment and product titer quantification (data not shown).

Experiments in this study were also conducted in TPP® TubeSpin bioreactor tubes (referred to shake tubes or $S T$ ) filled with supplement enriched proprietary medium according to table 6.1 in the absence of MSX. CHO-S cells were seeded at a viable cell density of $0.30 \times 10^{6}$ viable cells $/ \mathrm{mL}$ and CHO-K1 cells at $0.20 \times 10^{6}$ viable cells $/ \mathrm{mL}$. The tubes were incubated in a shaker incubator at $36.5{ }^{\circ} \mathrm{C}, 5 \% \mathrm{CO}_{2}, 80 \%$ humidity and $320 \mathrm{rpm}$ agitation (ISF1-X, Adolf Kühner, Birsfelden, Switzerland) for 14 days. Chemically defined feed containing over 30 components and alkaline amino acid solution were added on day 3, 5, 7 and 10, while the $400 \mathrm{~g} / \mathrm{L}$ glucose solution was added on these days and on day 12 in addition. Prior to each feeding and at the end of the culture (day 14), aliquots ( $\leq 2.5 \mathrm{~mL}$ ) were taken for viable cell counting, extracellular metabolite profiling and product titer determination (data not shown).

Table 6.1 - Concentration ranges of cell culture medium supplements in medium prior to inoculation.

\begin{tabular}{lcccc}
\hline Compound & Scale & Medium Component & Addition & Supplier \\
\hline Cysteine & $96-\mathrm{DWP}$ & Yes & $0-6 \mathrm{mM}$ & Sigma Aldrich \\
& ST & Yes & $0-50 \mathrm{mM}$ & Sigma Aldrich \\
Alanine & $96-\mathrm{DWP}$ & Yes & $0-12 \mathrm{mM}$ & Sigma Aldrich \\
Glycine & $96-\mathrm{DWP}$ & Yes & $0-12 \mathrm{mM}$ & Sigma Aldrich \\
Histidine & $96-\mathrm{DWP}$ & Yes & $0-12 \mathrm{mM}$ & Sigma Aldrich \\
Isoleucine & $96-\mathrm{DWP}$ & Yes & $0-12 \mathrm{mM}$ & Sigma Aldrich \\
Methionine & $96-\mathrm{DWP}$ & Yes & $0-12 \mathrm{mM}$ & Sigma Aldrich \\
Proline & $96-\mathrm{DWP}$ & Yes & $0-12 \mathrm{mM}$ & Sigma Aldrich \\
N-acetyl-cysteine & ST & No & $0-2 \mathrm{mM}$ & Sigma Aldrich \\
Ferric ammonium citrate & ST & Yes & $0-225 \mu \mathrm{M}$ & Merck \\
Cupric sulfate & ST & Yes & $0-225 \mu \mathrm{M}$ & Merck \\
Zinc sulfate & ST & Yes & $0-225 \mu \mathrm{M}$ & Merck \\
\hline
\end{tabular}

To further study the effect of cysteine, the amino acid was added throughout the culture as part of the feed instead of medium supplementation. The cell seeding density was set to $0.30 \times 10^{6}$ viable cells $/ \mathrm{mL}$ for cell line A and to $0.20 \times 10^{6}$ viable cells $/ \mathrm{mL}$ for cell line B. The exponentially growing cells were inoculated in proprietary medium only (in the absence of MSX). The high-throughput fed-batch cell culture was performed on a robotic liquid handling platform (Biomek FX, Beckman Coulter, Brea, CA). The plates were incubated with vented lids to minimize evaporation in a shaker incubator at $36.5{ }^{\circ} \mathrm{C}, 5 \% \mathrm{CO}_{2}, 90 \%$ humidity and $320 \mathrm{rpm}$ agitation (ISF1-X, Adolf Kühner, Birsfelden, Switzerland) for 14 days. 400 g/L glucose solution, 
chemically-defined feed containing over 30 components and alkaline amino acid solution were added on day 3, 5, 7, 10 and 12. Cysteine (Merck) concentrations in the chemicallydefined feed ranged between 0 and $30 \mathrm{mM}$ for cell line A and 0 and $50 \mathrm{mM}$ for cell line B. Prior to each feeding and at the end of the culture on day 14 , samples $(\leq 40 \mu \mathrm{L})$ were drawn for growth and viability assessment and product titer quantification (data not shown).

The effect of chelating agents ethylenediaminetetraacetic acid (EDTA) dimercaptosuccinic acid (DMSA) were studied at the end of the cell culture. They exhibited detrimental effects on the growth when added at the beginning or during the culture. To circumvent the toxic effects, the supernatant was supplemented at the end of culture on day 14 prior to harvesting with EDTA or DMSA at different concentrations according to table 6.2.

Table 6.2 - Concentrations of chelating agents added to the supernatant before harvesting (day 14).

\begin{tabular}{llll}
\hline Compound & Scale & Concentration Ranges & Supplier \\
\hline EDTA & ST & $0-2.2 \mathrm{mM}$ & Sigma Aldrich \\
DMSA & ST & $0-2.2 \mathrm{mM}$ & Sigma Aldrich \\
\hline
\end{tabular}

\subsubsection{Analytical Methods for Cell Culture Performance}

Growth and viability assessment of 96-DWP cultures was performed on a Guava easyCyte (Merck Life Science, Darmstadt, Germany), while for shake tube and 3.5-L bioreactor cultures a Vi-Cell analyzer (Beckman Coulter, Brea, CA). The product titer of the 96-DWP cultures was analyzed on day 14 by an Octet ${ }^{\circledR}$ (forteBIO, Menlo Park, CA), using Protein A sensors. Each sample was diluted 20-40 times into a dilution buffer ( $\mathrm{PBS} \mathrm{pH}=7.4, \mathrm{BSA} 1 \mathrm{~g} / \mathrm{L}$, Tween 20 at $0.02 \%$ ). The sensors were regenerated with aqueous buffer containing $10 \mathrm{mM}$ glycine$\mathrm{HCl}$ at $\mathrm{pH} 1.5$ and neutralized with the dilution buffer. Titer quantification of samples from shake tubes collected on day 14 was performed, using a Biacore C instrument (GE Healthcare, Waukesha, WI).

\subsubsection{Analysis of Low-Molecular-Weight Species Content}

At the end of each 96-DWP and ST fed-batch experiment (day 14), the supernatant was purified on small-scale affinity columns (PhytipsVR, PhyNexus, San Jose, CA), eluting in $20 \mathrm{mM}$ citric acid, $20 \mathrm{mM} \mathrm{PO}_{4}^{3-}$ buffer. Following neutralization in $0.5 \mathrm{M}$ Tris, the low-molecular-weight species content of the eluates was analyzed by LabChip GXII protein assay (PerkinElmer, Waltham, MA). All solutions mentioned hereafter were supplied by PerkinElmer, Waltham, MA. The chip and the reagents were equilibrated at room temperature for 20-30 minutes. The protein express gel matrix was added into the protein express dye solution to prepare the gel-dye. For destain solution preparation, the protein express gel matrix and the gel-dye were 
centrifuged at $9300 \mathrm{rcf}$ for 5 minutes at RT. Each well was rinsed and aspirated twice with ultra-pure water. Then both the destain solution and the gel-dye were added onto the chip. In addition, the protein express lower marker was added. Both sides of the chip window were cleaned with the supplied clean room cloth dampened with $70 \%$ isopropanol. The denaturing solution was obtained, mixing dithiothreitol with protein express sample buffer. The Phytips eluates were added into the denaturing solution. The protein express ladder was subsequently transferred to a microcentrifuge tube. The samples and the ladder were denatured at $100{ }^{\circ} \mathrm{C}$ for 5 minutes. At this time, ultra-pure water was added to the samples and to the ladder and both were mixed. After sample transfer to a microtiter plate, the prepared latter was transferred to the provided ladder tube. Finally, protein express wash buffer was added. The analysis was performed, using LabChipGX (PerkinElmer, Waltham, MA)

\subsection{Results}

\subsubsection{Amino Acids}

As part of a broader amino acid screening, it was observed that cysteine concentration in the medium correlated with the level of low-molecular-weight species in cell line 96-DWP cultures. Figure 6.1A shows that the LMW content ranged between 1.4 and $2.1 \%$ with no additional cysteine supplementation $(0 \mathrm{mM})$. It is not clear whether this range corresponds to the natural process variation. Analytical variability amounts to $2.4 \%$, and therefore, does not explain this wide range. It is also possible that increased levels of one of the amino acids alanine, glycine, histidine, isoleucine, methionine, and proline contributed to the variation at the low cysteine level. Nonetheless, higher cysteine levels in the cell culture medium exhibited a tendency of more abundant LMW formation. A concentration difference of $6 \mathrm{mM}$ favored the fragmentation of the expressed antibody. The LMW levels ranged between 2.6 and $2.8 \%$. The goodness of fit of the linear regression $\left(R^{2}\right)$ amounted to $61.9 \%$ and its slope was statistically significant ( $p$-value $=0.000$ ). The experiment was repeated, adding the cysteine during the culture in the CD-feed. Likewise, the cysteine feed supplementation was part of a global amino amino acid screening experiment. At $0 \mathrm{mM}$, the LMW varied between 3.7 and $6.2 \%$ (figure 6.1B). Hence, glycine, proline, serine, tyrosine, threonine, may also have induced a greater variation. Once again, the LMW levels tended to climb when more cysteine was added. For instance, at $25 \mathrm{mM}$, LMW varied between 5.1 and $8.5 \%$. The goodness of fit of the linear regression $\left(R^{2}\right)$ was $52.4 \%$. The slope of the line was significant ( $\mathrm{p}$-value $=0.000$ ).

To further study whether this increasing trend was limited to one cell line or if a similar effect may be observed in another as well, a screening experiment was performed with cell line B in 96-DWP. In figure 6.2A, a tendency towards higher LMW levels with higher cysteine concentrations can be noticed. If no additional cysteine is added, LMW varied between 2.1 and $3.2 \%$. Like in the above described experiment with cell line A, at this cysteine level the medium contained higher levels of one of the following amino acids: alanine, glycine, histidine, 
A

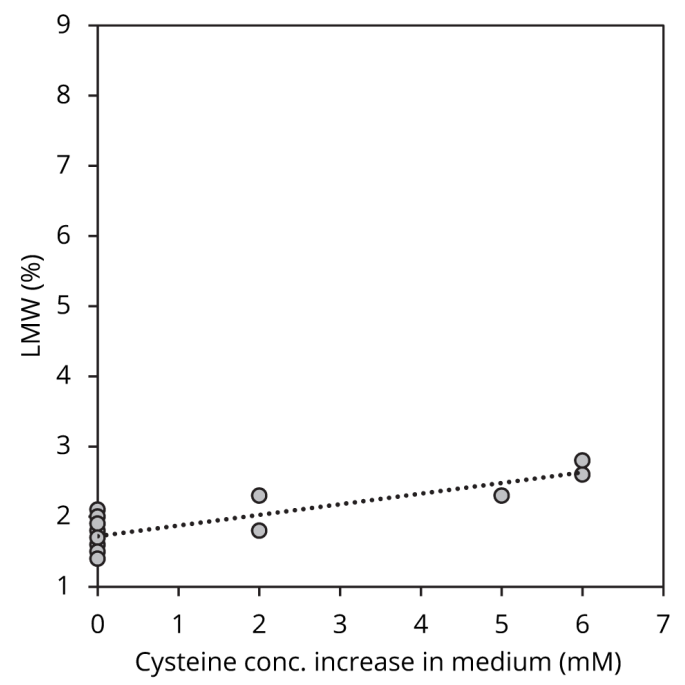

B

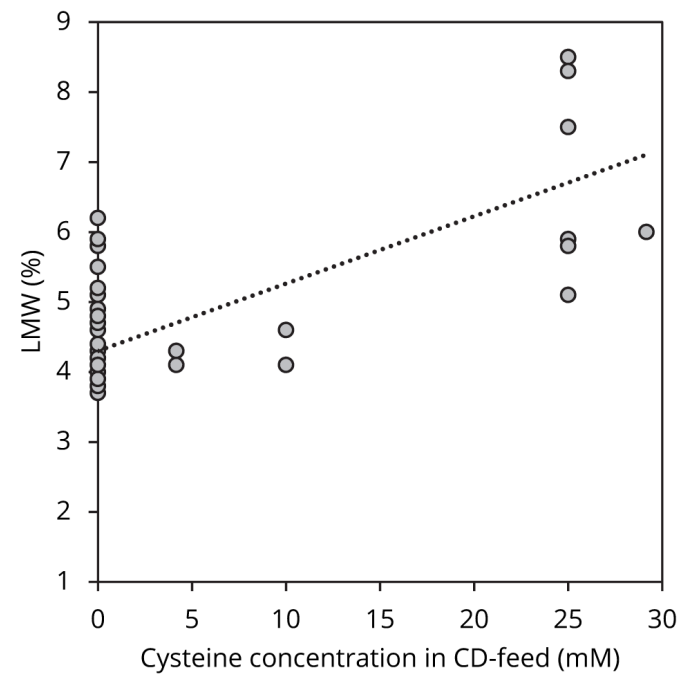

Figure 6.1 - (A) LMW levels of cell line A cultures in 96-DWP in function of the cysteine concentration increase in the medium prior to inoculation, including the linear regression line (equation: $\operatorname{LMW}(\%)=1.722+0.1517$ cysteine $\left.(\mathrm{mM}), R^{2}=61.9 \%\right)$. (B) LMW levels of cell line A cultures in 96-DWP in function of the cysteine concentration in the CD-feed added on days 3 , $5,7,10$, and 12. The linear regression line is shown as well (equation: $\operatorname{LMW}(\%)=4.405+0.09167$ cysteine (mM), $\left.R^{2}=52.4 \%\right)$.

isoleucine, methionine, and proline. They might have contributed to the LMW variation. Supplementation of the culture medium and thus an increase of the cysteine concentration by $6 \mathrm{mM}$ moved the LMW range to $3.1-3.9 \%$. Despite a rather poor fit $\left(R^{2}=36.4 \%\right)$, the linear regression does show an upward trend of LMW with increasing cystein concentrations. The slope of the trend-line was significant ( $\mathrm{p}$-value $=0.000$ ). Figure $6.2 \mathrm{~B}$ presents the LMW increase in the case of feed supplementation. At the exception of the outliers in the range of about $11 \%$, the same trend was observed. No presence of cysteine during the culture resulted in LMW levels of 3.6 to $5.0 \%$. These conditions also contained, as in cell line A experiments, higher levels of other amino acids. Feeds containing $50 \mathrm{mM}$ brought about higher LMW levels: 5.8 to $6.6 \%$. The goodness of fit of the linear regression is poor, taking into account the two outliers: $R^{2}=19.7 \%$. However, if they were removed, the quality of the regression greatly improved, reaching $79.8 \%$. The slope of the regression line was significant $(p$-value $=0.000$ ).

Interestingly, both cell lines featured a correlation between the level of fragmentation and the final product titer. Figure 6.3 shows a negative slope of the linear regressions of the harvest titers in various 96-DWP experiments of both cell lines. Increasing titer correlated with reduced generation of low molecular species. In cell line A cultures the regression line crossed the $2 \%$ LMW level at mAb titers of about $1000 \mathrm{mg} / \mathrm{L}$. In the range of 2000 to $2500 \mathrm{mg} / \mathrm{L}$, it ended up at $1.5 \%$. The three substantially higher points at a product titer of about $1000 \mathrm{mg} / \mathrm{L}$ were experimental conditions with increased cystein concentrations (gray circle). The goodness 
A

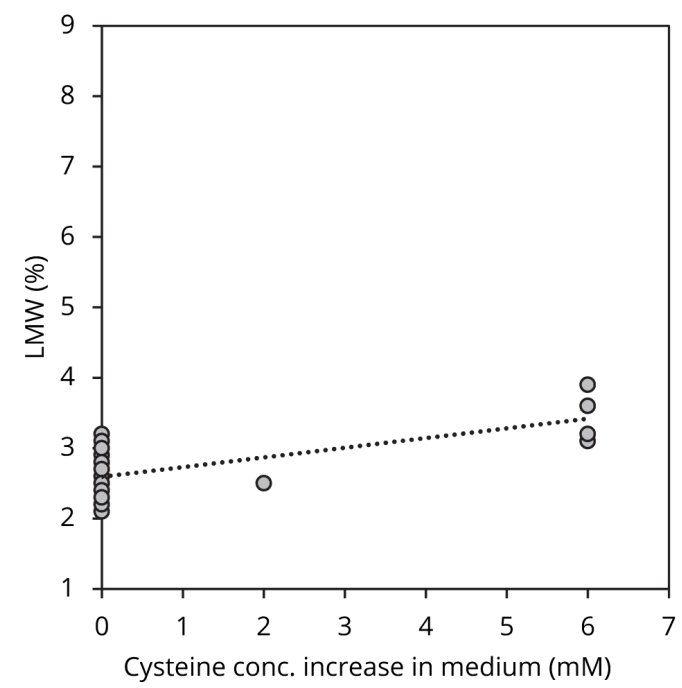

B

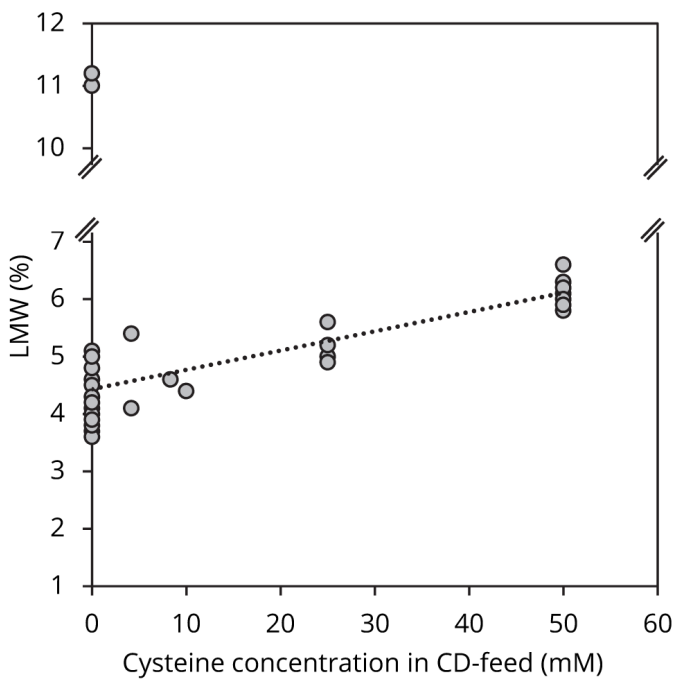

Figure 6.2 - (A) LMW levels of cell line B cultures in 96-DWP in function of the cysteine concentration increase in the medium prior to inoculation, including the linear regression line (equation: $\operatorname{LMW}(\%)=2.593+0.1377$ cysteine $\left.(\mathrm{mM}), R^{2}=36.4 \%\right)$. (B) LMW levels of cell line B cultures in 96-DWP in function of the cysteine concentration in the CD-feed added on days 3 , $5,7,10$, and 12 . The linear regression line is shown as well (equation: $\operatorname{LMW}(\%)=4.434+0.03361$ cysteine $\left.(\mathrm{mM}), R^{2}=19.7 \%\right)$.

of fit of the linear regression $\left(R^{2}\right)$ amounted to $40.7 \%$. Based on the statistical analysis, it can be concluded that the slope of the regression line is significant: $p$-value $=0.000$. Cell line B exhibited a similar pattern. The trend-line was located in the region of $2.8 \%$ at a harvest titer of $1000 \mathrm{mg} / \mathrm{L}$ and reached a level of LMW of about $2.2 \%$ at $3000 \mathrm{mg} / \mathrm{L}$. Likewise, the three substantially higher points (LMW $\geq 3.1 \%$ ) were experimental conditions with increased cystein concentrations (gray circle). The $R^{2}$ of the linear regression was rather low, amounting to $25.9 \%$. If the three cystein outliers are removed, the goodness of fit increases substantially: $48.2 \%$. Nonetheless, despite the presence of these outliers, the statistical analysis shows that the slope of the regression line was significant: $p$-value $=0.000$.

A larger cysteine concentration range was tested (figure 6.4) when scaling up the cell line B process to ST. The non-supplemented control cultures yielded on average a LMW level of $4.3 \%$. With higher cysteine concentrations (increases by 25 and $50 \mathrm{mM}$ ), the LMW levels climbed to $5.9 \%$ and to $6.3 \%$ on average. The most pronounced increase of LMW resulted when increasing the cysteine from 0 to $25 \mathrm{mM}$. While the LMW levels between 25 and $50 \mathrm{mM}$ still augmented, the amino acid produced a considerably smaller LMW increase at higher concentration. Overall, the relationship between the cysteine concentration in the supernatant and the level of fragmentation observed in 96-DWP was thus confirmed in ST. Figure 6.5 shows the electropherogram of one control culture (A) and one culture supplemented with $50 \mathrm{mM}$ (B). Two major fragmented species can be distinguished. The peak at 0.337 minutes corresponds to one 
A

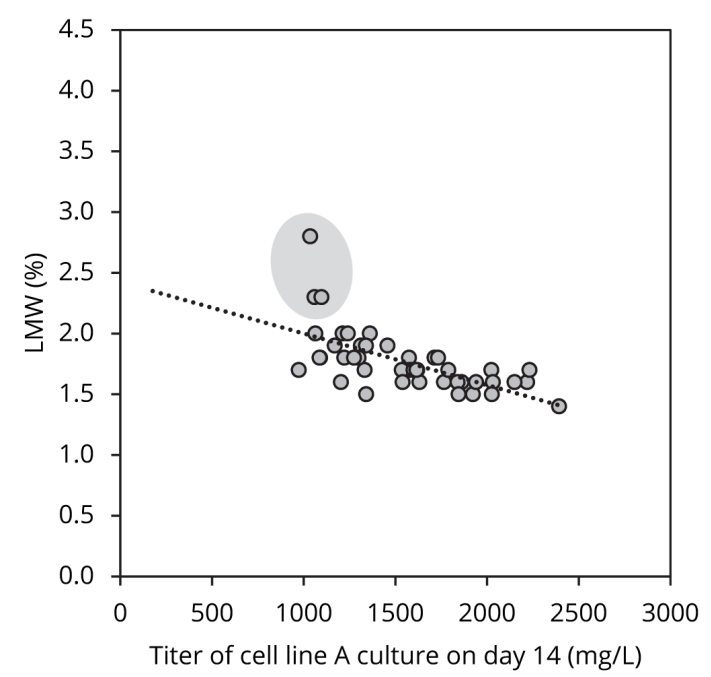

B

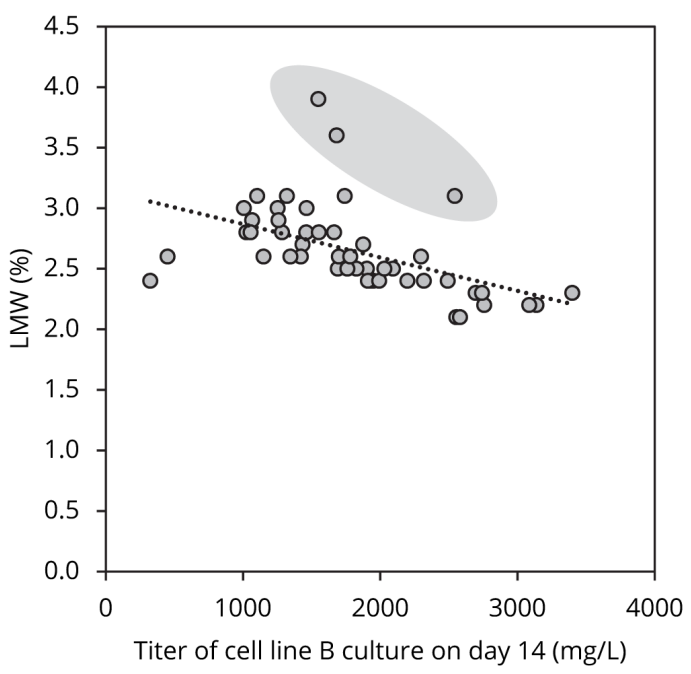

Figure 6.3 - (A) LMW content cell line A cultures in 96-DWP in function the cell line A protein titer at the end of the culture (day 14), including the linear regression line (equation: $\mathrm{LMW}(\%)=$ $2.425-0.000424$ titer $\left.(\mathrm{mg} / \mathrm{L}), R^{2}=40.7 \%\right)$. The cysteine supplemented cultures are highlighted (gray circle). (B) LMW content cell line B cultures in 96-DWP in function the cell line A protein titer at the end of the culture (day 14), including the linear regression line (equation: $\operatorname{LMW}(\%)=$ $3.145-0.000276$ titer $\left.(\mathrm{mg} / \mathrm{L}), R^{2}=25.9 \%\right)$. The cysteine supplemented cultures are highlighted (gray circle). 
single light chain (L), while the peak at 0.490 minutes corresponds to the antibody including two heavy chains and one light chain thus lacking one light chain: HHL. Comparing the two charts, one cannot visually see the increase of LMW in the supplemented culture. Only after peak integration the LMW difference can be appropriately quantified. Nonetheless, what can be seen is that no additional peaks appeared in the supplemented culture. Hence, the cysteine presence only increased the abundance of species already present in the non-supplemented culture and thus did not generate new species.

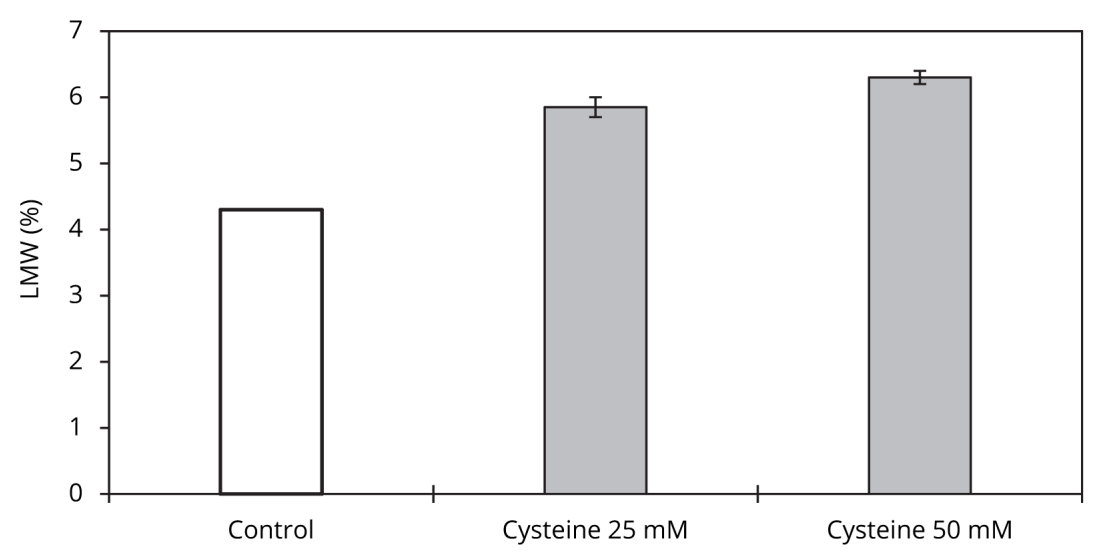

Figure 6.4 - LMW content in function of the cysteine concentration increase in the medium prior to inoculation. Each condition was performed in duplicates. All bars represent mean values of the corresponding conditions and the error bars report the maximum and minimum values.

\subsubsection{N-Acetyl-Cysteine}

$\mathrm{N}$-acetyl-cysteine (NAC) medium supplementation in the range of 0-2 $\mathrm{mM}$ affected the level of LMW in cell line B cultures in ST. According to figure 6.6, 3.9\% of the expressed antibody were fragmented on average. The degree of cleavage slightly increased to $4.4 \%$ at a NAC concentration of $0.29 \mathrm{mM}$. At the highest NAC level $(2 \mathrm{mM})$, fragmentation was further promoted, reaching a LMW level of 4.9\%. It is not clear why the condition at $1 \mathrm{mM}$ NAC produced less LMW than at $0.29 \mathrm{mM}$. But, this experiment suggest that an overall increasing trend of fragmentation correlated with increasing NAC concentration in the supernatant. Figure 6.7 shows the electropherogram of the corresponding analysis. Like in the case of cysteine, the most abundant fragmented species were one single light chain and the antibody lacking one light chain. No new peaks, and as a consequence, no new fragmented entity arose in the supplemented culture. 


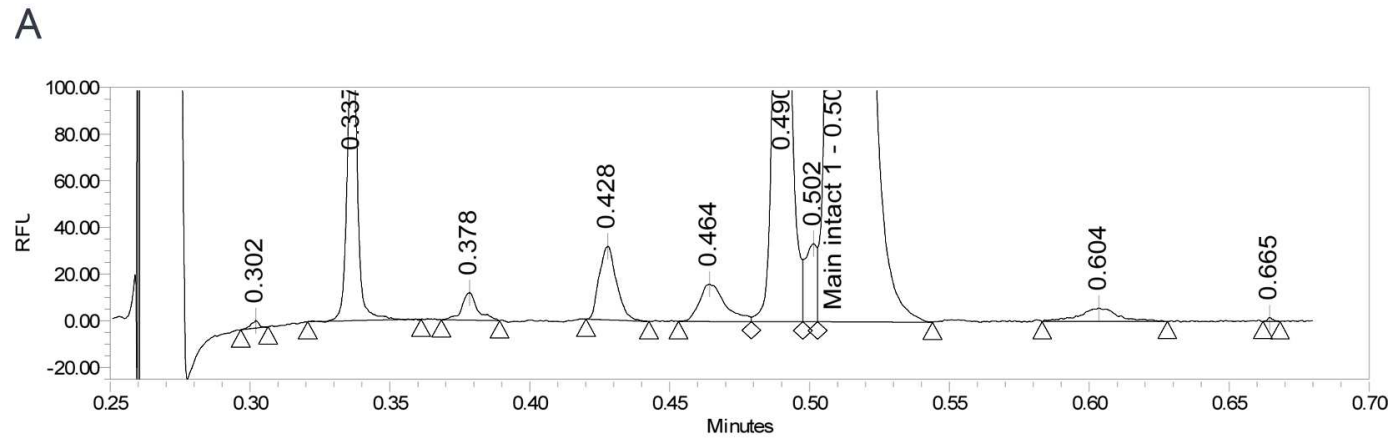

B

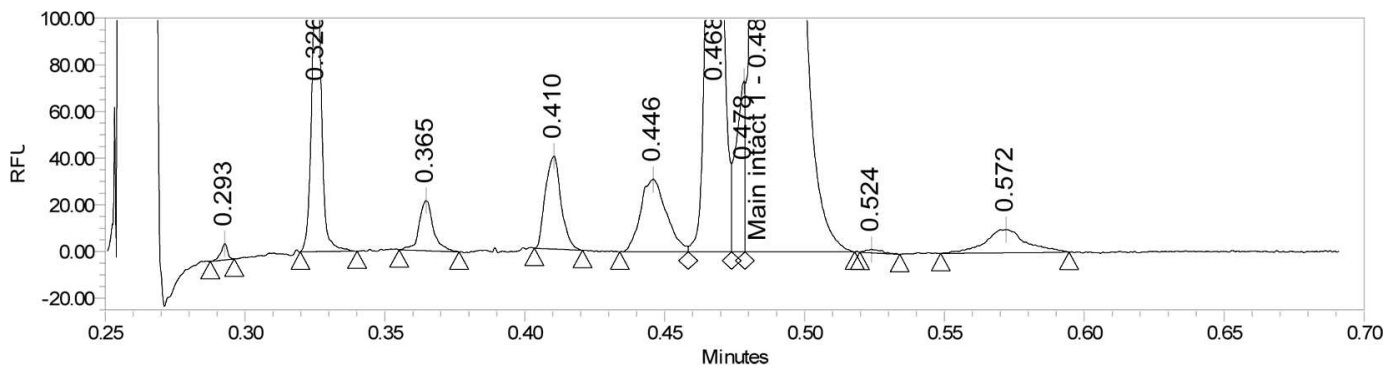

Figure 6.5 - Electropherogram of one control sample and one of the culture supplemented with $50 \mathrm{mM}$ cysteine.

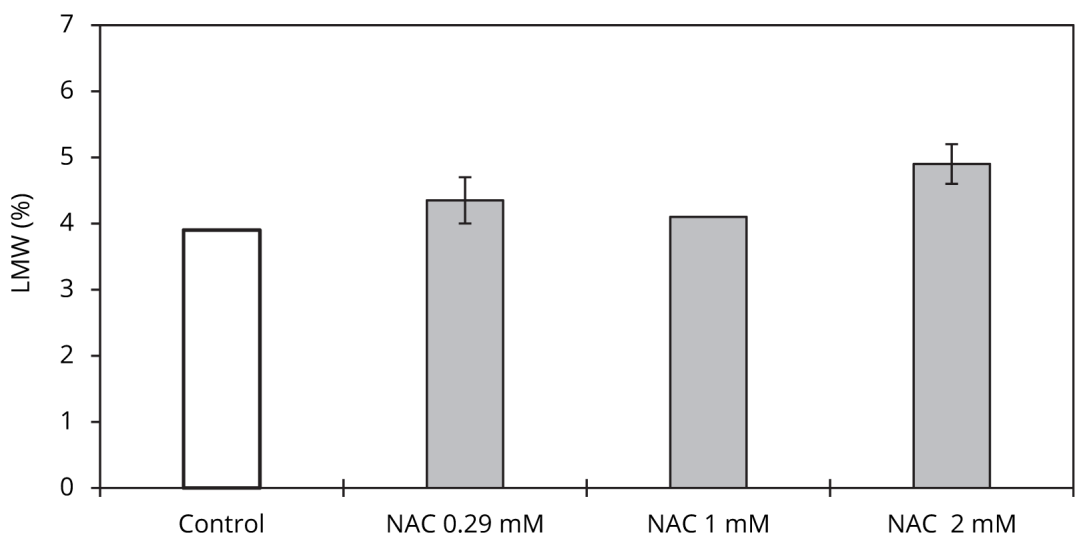

Figure 6.6 - LMW content in function of the $\mathrm{N}$-acetyl-cysteine concentration in the medium prior to inoculation. Each condition was performed in duplicates. All bars represent mean values of the corresponding conditions and the error bars report the maximum and minimum values. 
A

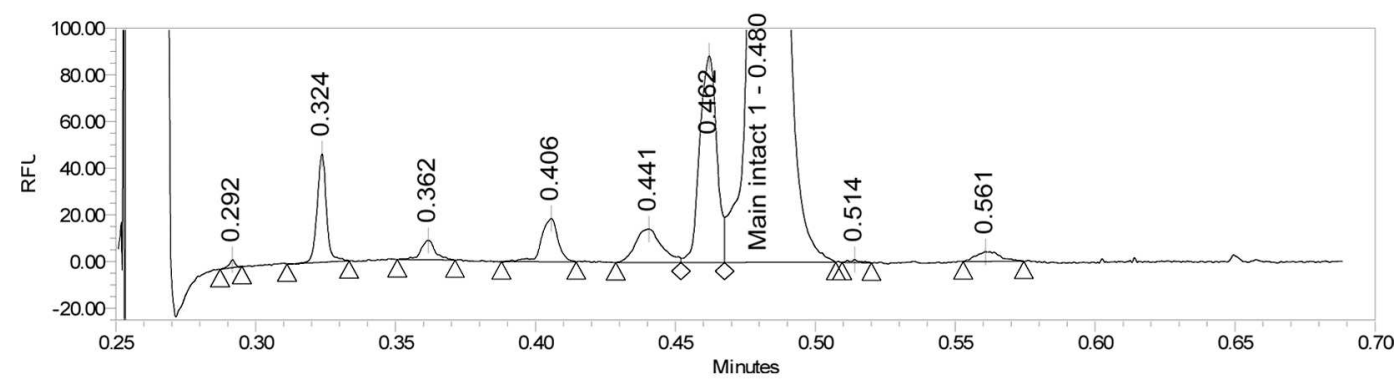

B

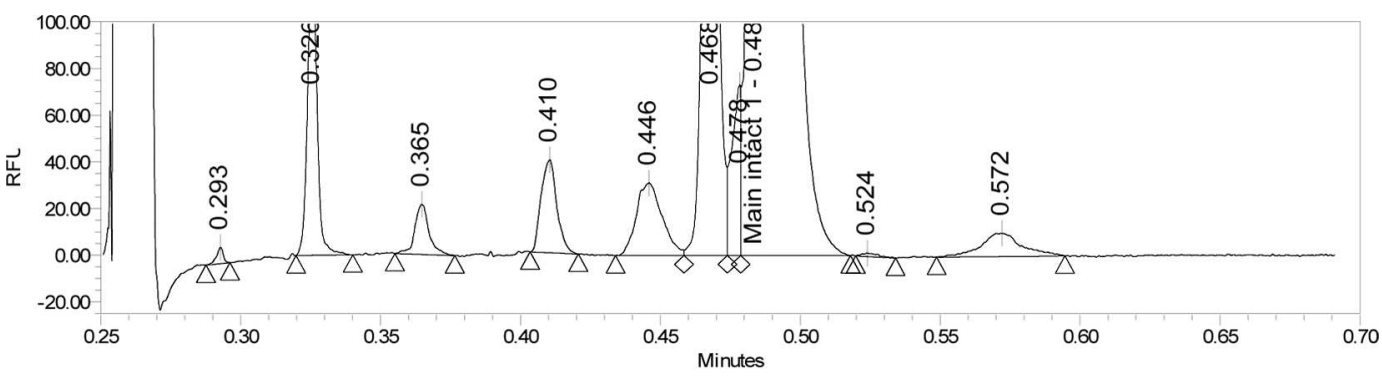

Figure 6.7 - Electropherogram of one control sample and one of the culture supplemented with $2 \mathrm{mM} \mathrm{N}$-acetyl-cysteine. 


\subsubsection{Chelating Agents}

The use of EDTA, a widely used chelating agent ${ }^{252}$, in cell culture, entailed small effects on the LMW formation (figure 6.8). 3.7\% of the control culture antibodies underwent cleavage. It is important to note that the control was analyzed in a separate analytical sequence than the remainder of the samples. While the analytical variability within one analytical sequence is small (2.4\%), a relative standard deviation (RSD) of $31.5 \%$ applies between sequences, which is due to unidentified inter-assay factors. Hence, it cannot be concluded whether the addition of EDTA induced a change of the LMW level. However, it is possible to compare the effects of EDTA and DMSA as they were part of the same analytical sequence. At 0.56 and $2.2 \mathrm{mM}$ EDTA 4.5 and $4.3 \%$ LMW were present in the supernatant. In the tested EDTA concentration range, EDTA did not exhibit any impact on the cleaving process of antibodies. A different picture emerged with DMSA. At a DMSA concentration of $0.56 \mathrm{mM}$ in the medium prior to inoculation, the level of LMW doubled in comparison with EDTA. It amounted to 8.8\%. Further supplementation increasing the concentration to $2.2 \mathrm{mM}$ resulted in $11.9 \%$ of cleaved species. Figure 6.9 shows that DMSA supplementation principally resulted in larger and taller L and HHL peaks. The HH at 0.463 minutes in figure 6.9B became slightly larger. The HL peak at 0.428 minutes as well. Interestingly the substantially higher percentage of LMW species was mainly due to more abundant L- and HHL-fragments.

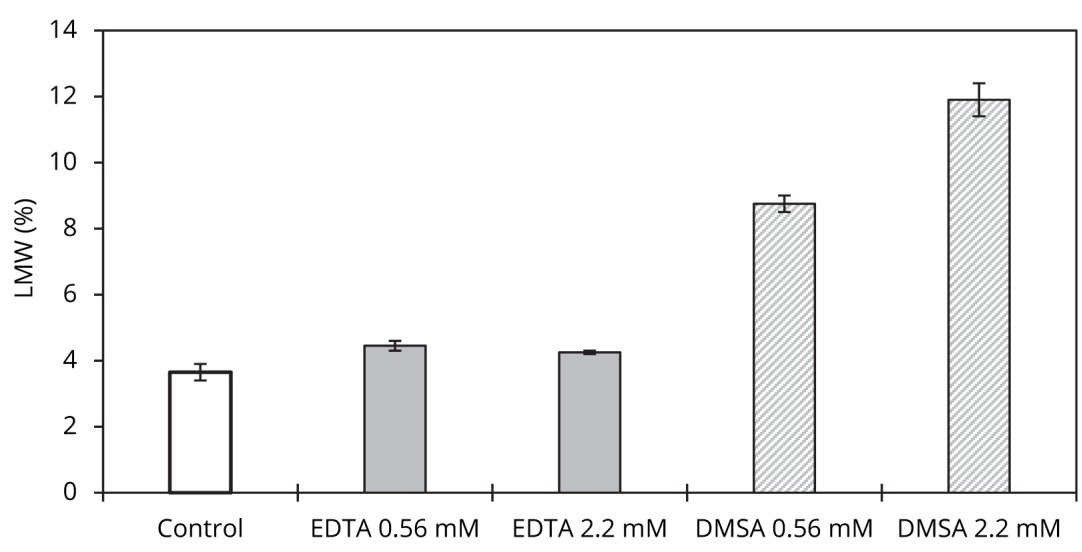

Figure 6.8 - LMW content in function of the EDTA and DMSA concentrations in the medium prior to inoculation. Each condition was performed in duplicates. All bars represent mean values of the corresponding conditions and the error bars report the maximum and minimum values. The control culture was analyzed in a separate sequence than the remainder of the supplemented conditions.

\subsubsection{Metal Ions}

Figure 6.10 summarizes the influence of the iron concentration in the medium on fragmentation. Its level in the control cultures at a ferric ammonium citrate concentration increase $83 \mu \mathrm{M}$ with respect to the control was comparable. On average, both the control and the 
A

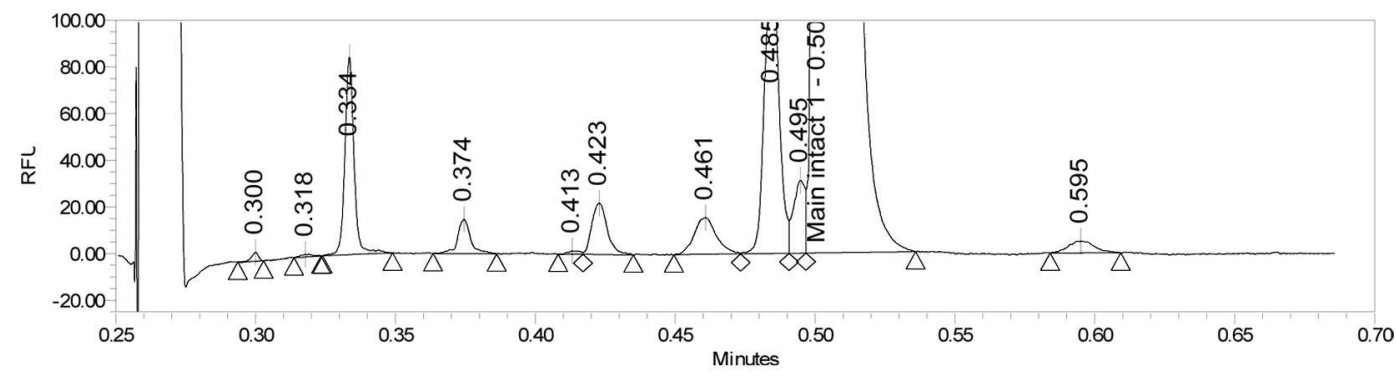

B

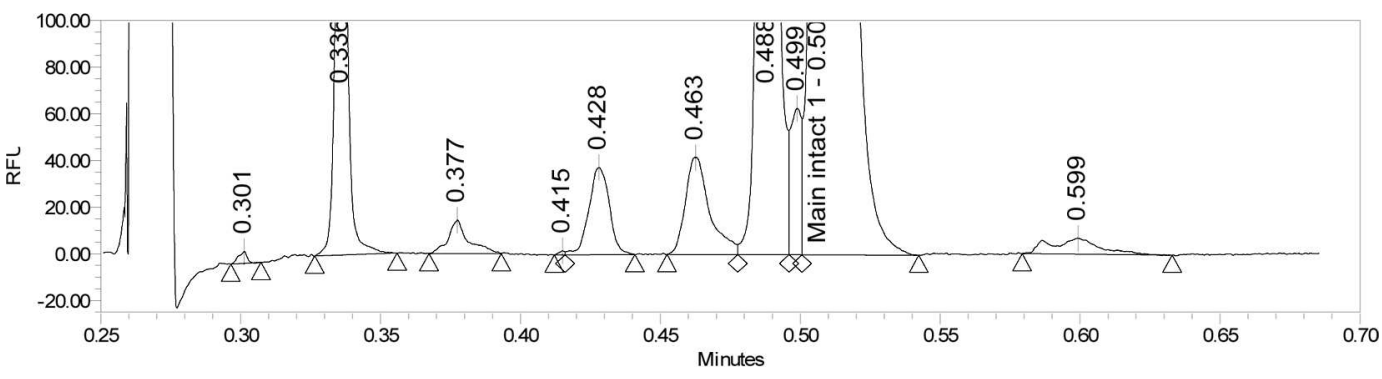

Figure 6.9 - Electropherogram of one control sample and one of the culture supplemented with $2.2 \mathrm{mM}$ DMSA. 
supplemented culture contained 1.5\% LMW. A ferric ammonium citrate increase by $225 \mu \mathrm{M}$ slightly favored protein cleavage. At culture harvest, the LMW level reached 1.9\%. Due to overall small peaks of the fragmented species in this analytical sequence (figure 6.11), one cannot visually identify significant changes. Iron supplementation did not effect new fragmented species in comparison to the cultures performed in non-supplemented medium.

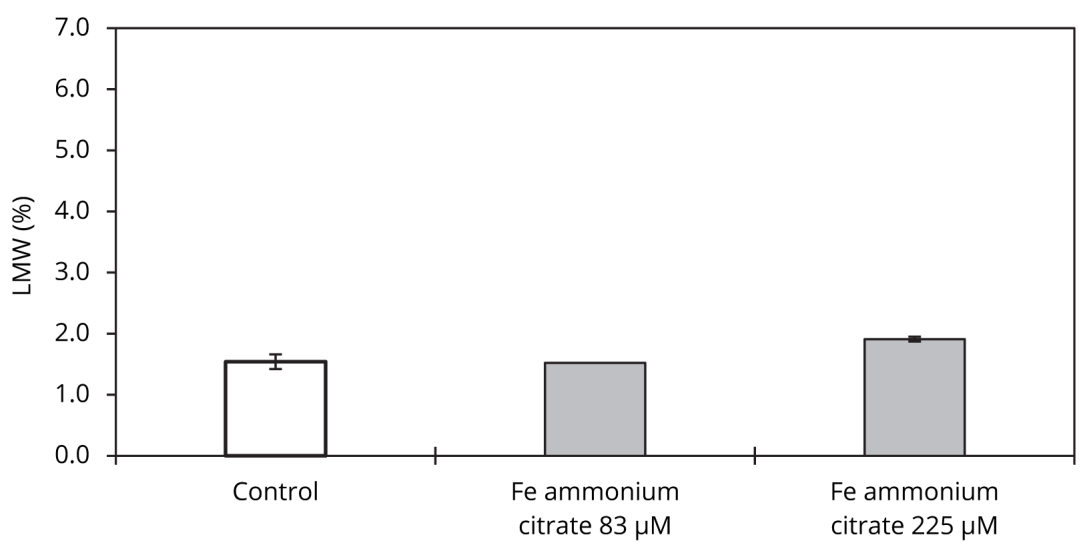

Figure 6.10 - LMW content in function of the ferric ammonium citrate concentration increase in the medium prior to inoculation. Each condition was performed in duplicates. All bars represent mean values of the corresponding conditions and the error bars report the maximum and minimum values.

Moreover, the effect of further metals already part of the medium formulation including copper and zinc was assessed. In the $\mathrm{CuSO}_{4}$ and $\mathrm{ZnSO}_{4}$ concentration ranges of our study, no significant changes of the LMW were observed (figure 6.12). The average LMW content in the control and at both 83 and $225 \mu \mathrm{M}$ of the respective supplement remained in the range of 1.5 to $1.6 \%$.

\subsection{Discussion}

Globally, the induced LMW level changes were small. The important preceding optimization efforts of the proprietary fed-batch process platform enable to maintain high viabilities, in general greater than $80 \%$ at the end of the culture of a 14 -day run ${ }^{19,25,251}$. As a result the current cell-culture platform process delivers consistently high titers and rather low aggregate and lowmolecular-weight species. However, even if fragmentation is relatively small in comparison to the expressed monomers, the reduction thereof was important to tackle for the proprietary purification platform does not remove them unlike efficient reduction of aggregates. Albeit lacking insights of the particular mechanisms underlying LMW generation in the proprietary platform process, it was possible to produce sufficiently large variations in the 96-DWP highthroughput screening platform. The cysteine supplementation in both the medium prior to inoculation and in the feed shows that the 96-DWP processes yielded LMW-level responses 
A

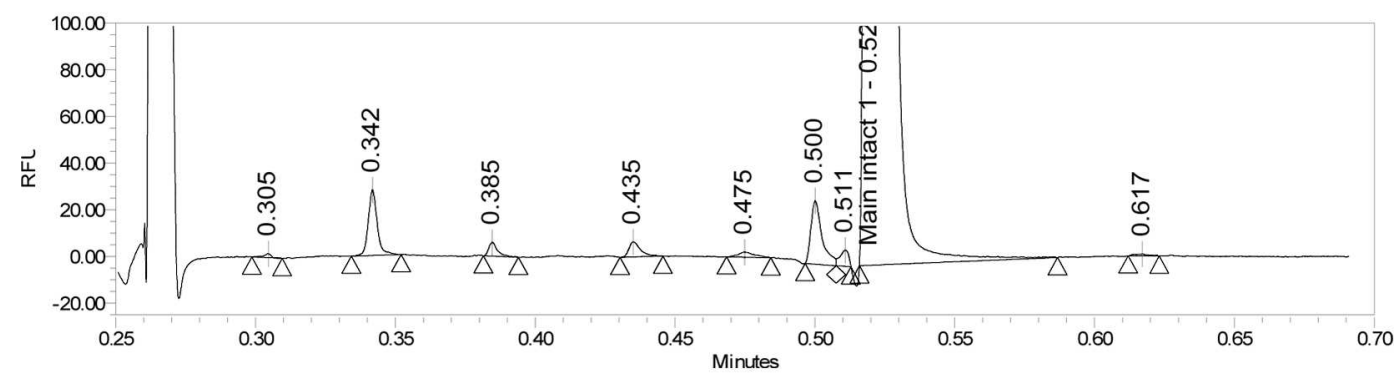

B

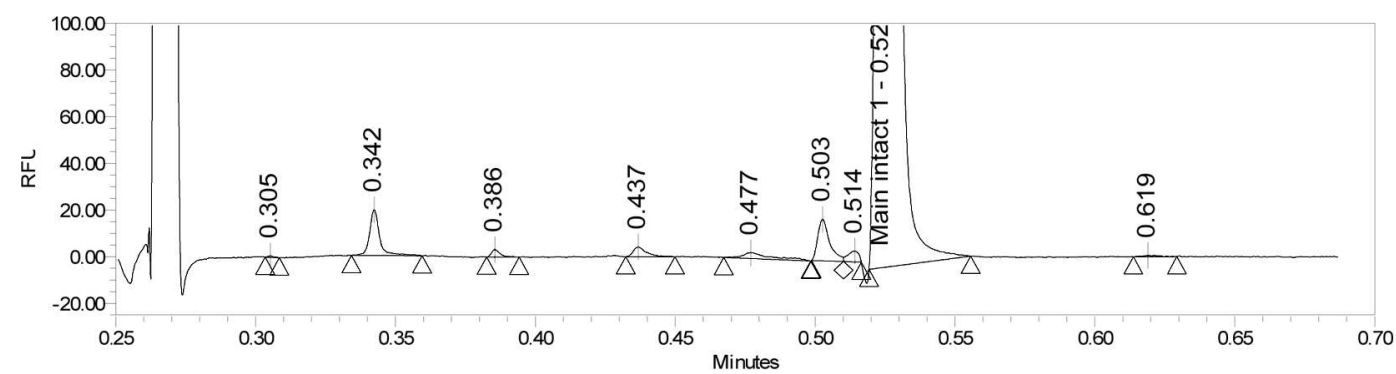

Figure 6.11 - Electropherogram of one control sample and one of the culture supplemented with $225 \mu \mathrm{M}$ ferric ammonium citrate.

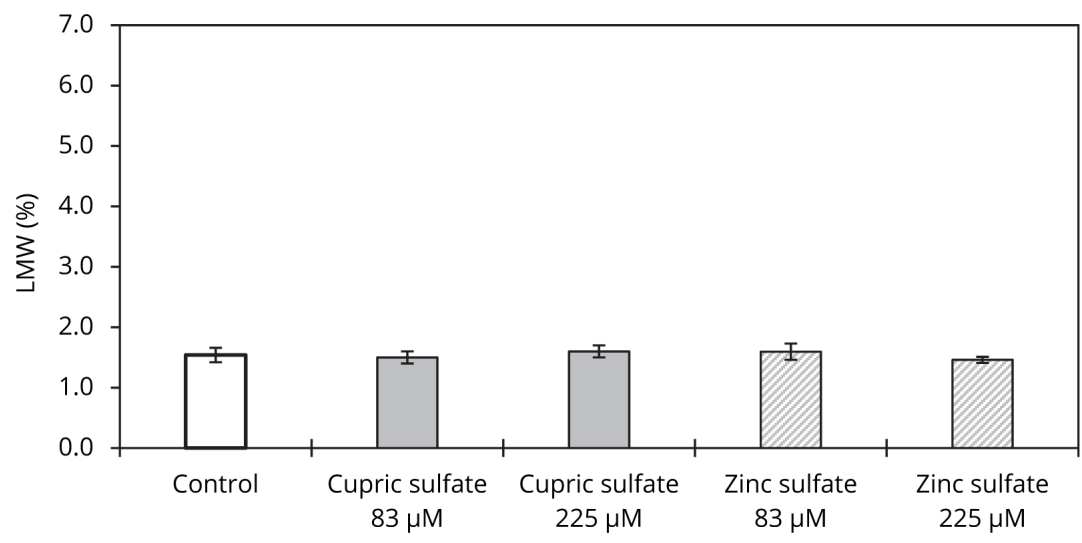

Figure 6.12 - LMW content in function of the $\mathrm{CuSO}_{4}$ and $\mathrm{ZnSO}_{4}$ concentration increases in the medium prior to inoculation. Each condition was performed in duplicates. All bars represent mean values of the corresponding conditions and the error bars report the maximum and minimum values. 
that were greater than the analytical variability. The process variability was high, especially due to the fact that many different amino acids were tested simultaneously. Nevertheless, an increasing trend of LMW with increasing cysteine concentration was observed in two different cell lines. At that stage of the development no quantitative changes were looked for. The 96-DWP provided the opportunity to test a great number of different conditions in order to identify supplements and/or concentration ranges that influence the LMW content. Although the process variability was high, the resulting differences were statistically significant. Concentration increases of cysteine in the medium by $6 \mathrm{mM}$ induced on average about $0.8 \%$ higher LMW levels in both cell lines, A and B. The amplitude of the LWM level change that feed supplementation experiments induced ranged between $1.7 \%$ (cell line B) and $2.8 \%$ (cell line A). Even when supplementing the medium, the 96-DWP model was good enough to produce qualitative results, allowing to discriminate between the various amino acids whether they influenced fragmentation or not in the experimental conditions of the study. And this was the case although some did not grow well and not produce sufficient protein for LMW quantification. No trend was observed in the presence of the other amino acids with no cysteine supplementation (data not shown). It is important to mention that the goodness of fit of the linear regression was relatively poor, which becomes evident when considering a $R^{2}$-values ranging between 20 and $62 \%$. The 96-DWP scale can be considered by no means as a reliable tool to identify solid correlations with weak responses. Nonetheless, one has to bear in mind, that the 96-DWP is a tool allowing to perform cell culture experiments at very small volumes, and due to the few controlled process parameters, greater variability resulted. On the other hand, the greater number of experiments still provided meaningful results for further confirmation studies in more controlled cell-culture systems.

Furthermore, the 96-DWP scale was a great tool to detect the correlation between the harvest titer (on day 14) and the degree of fragmentation. This high-throughput method allows a greater number of experimental conditions than in larger-scale systems, such as ST and microscale bioreactor systems (e.g. AMBR®), but small differences would be much more difficult to detect. Both cell lines (A and B) produced lower levels of fragmentation with increasing productivity. Interestingly, this pattern was preserved in the presence of cysteine too. In particular, LMW significantly increased at higher cysteine concentration, but even then, a downward trend in cell line B was noticed. With cysteine, the highest LMW levels were reached at the lowest harvest titer. At the moment, it was not possible to confirm any mechanism explaining this observation. It is hypothesized that one potential explanation might be the increased ratio between proteases and the secreted $\mathrm{mAb}$ in the supernatant at lower titers. Hence, the enzymes would be more easily available. In the literature it was reported that free light chains in culture media correlated with antibody productivity and quality ${ }^{47}$. There is also evidence that the quality of the folding process was linked with the aggregate content, and indirectly with $\mathrm{LMW}^{249}$. While no clear trend between the product titer and LMW was observed in their work, the LMW formation decreased with increasing specific productivity. The results of the present study show an inversely proportional correlation between the product titer and the level of LMW. It is suggested to explore whether cell cultures yielding higher titers benefited 
from better quality of the protein expression. Hence, it is imperative to further investigate the underlying mechanisms.

The experiments in ST at a wider cysteine concentration range in cell line B (0-50 mM) confirmed the effect of this sulfur-containing amino acid. In comparison to the preliminary screening in 96-DWP, the ST experiments induced a stronger LMW increase. Cysteine medium supplementation produced differences of about $2 \%$. The inter-replicate variability was low: $\pm 0.15 \%$ at the most, which is the same order of magnitude as the analytical variability. The chosen strategy to perform preliminary screening by means of medium blending in a highthroughput mode before scaling up and targeting the range to suitable concentrations proved to be an efficient way to reliably identify cysteine as being one factor effecting higher abundance of fragmented antibodies. Due to its instability in the medium, cysteine readily oxydizes to its dimer, cystine, which is taken up by the cells, and then the disulfide bridge disintegrates in the cell ${ }^{253}$. N-acetyl-cysteine (NAC) - a precursor of cysteine for glutathione synthesis ${ }^{254}$-is converted by an enzymatic reaction to cysteine inside the cell $^{255}$, or subsequently extracellular deacetylation of NAC it enters the cell in the form of cysteine ${ }^{256}$. N-acetyl-cysteine supplementation favored LMW formation, already at considerably lower concentrations than cysteine. At 2 mM NAC, fragmentation ended up $1 \%$ above the control, while at a tenfold greater cysteine concentration increase $(25 \mathrm{mM})$ entailed a difference of $1.6 \%$. NAC might have more readily entered the cytosol thus the intracellular cysteine concentration was higher, favoring disulfide bridge cleavage. As a next step, it is planned to increase the NAC medium concentration to further study its influence on fragmentation.

EDTA supplementation at the end of the culture did not show any effect on fragmentation. Unfortunately, EDTA supplementation during the culture either in the medium or in the feed had detrimental effects on the viability of cells. After addition the cells died, and consequently, it was not possible to study the effect of this chelating agent on trace metals, which are involved in the TrxR and glutathion pathways. Optimisation of EDTA concentration and feed timing are required to circumvent toxic effects. DMSA considerably increased fragmentation. Rather than acting as a chelating agent, DMSA very likely had a similar effect than mercaptoethanol, a well-known protein-reducing agent ${ }^{257}$. Thus, the electropherograms suggest that the disulfide bond cleavage may have been promoted in the presence of DMSA. It principally generated more L- and HHL-fragments. No additional peaks appeared.

Among the metal ions, only iron influenced the degree of cleavage. The absence of any effect by copper or zinc may be interpreted in various ways. Possibly, the concentration range was not optimal. While increasing concentration may be an option, it would be more appropriate to remove the metal concentrations in the medium and to perform a screen in a wider concentration range. By doing so, potential masking effects due to saturation would be avoided. The understanding of the metal ions in the observed fragmentation is pivotal. This piece of information will enable to further understand the specific fragmentation mechanisms, using the proprietary cell culture platform process. There is evidence that $\mathrm{CuSO}_{4}$ addition at harvest inhibits fragmentation of antibodies ${ }^{55}$. 
The tests presented in this chapter were part of a wider exploratory study. Hence, a streamlined approach for sample preparation and analysis was used. For this reason, all supernatants were captured by Phytips prior to the LMW analysis. While the Protein A resin mainly interacts with the Fc-part, weak interactions between the light-chain fragments were observed (data not shown). These are sufficient to detect the light chain, using the LMW assay described in section 6.2.4. Nevertheless, it was taken into account that the majority of non-Fc fragments are lost. Because this study focused on the global LMW generation, the described method was considered fit for purpose. Future studies addressing individual fragments will require alternatives to Protein A purification prior to analysis in order to conserve the fragment variety. The entire array of supplements that were tested in the scope of this study generated predominantly L- and HHL-fragments. Therefore, disulfide bridge reduction may likely be the origin of the antibody disintegration. Future studies, without Protein A purification, shall also show whether other fragmentation mechanisms exist. The intracellular thioredoxin system plays an important role in the protein disulfide bond reduction ${ }^{186,258}$. The mammalian thioredoxin system comprising thioredoxin reductase (TrxR), the thioredoxin (Trx) and NADPH generate reduced thioredoxin that catalyzes disulfide bond reduction in a great number of proteins ${ }^{259}$ Selenium, a culture medium component, may favor this pathway as a result of the higher availability of the trace element during the formation of the selenocysteine residue of TrxR. On the other hand, trace elements including $\mathrm{Hg}^{2+}, \mathrm{Cu}^{2+}, \mathrm{Zn}^{2+}, \mathrm{Co}^{2+}$ and $\mathrm{Mn}^{2+}$, have been reported to form complexes with thiols and selenols and thus inhibit TrxR or $\operatorname{Trx}{ }^{186}$. Hence, it is suggested to further ascertain the role of $\mathrm{Cu}^{2+}, \mathrm{Zn}^{2+}$ by extending the concentration ranges of future experiments. The second intracellular enzymatic system, glutaredoxin (GrxR), exclusively reduces disulfide bonds of S-glutathionylated entities ${ }^{260}$. One may assume that the Trx system is therefore probably the principal intracellular enzymatic system responsible for the reduction of the antibody inter-chain disulfide bridges, which has been previously reported ${ }^{186}$. In addition, it shall still be studied whether, and if applicable, how extracellular enzymatic reactions further increase disulfide bond reduction while the protein resides in the cell culture fluid until harvest.

Media blending of fed-batch cultures in 96-deepwell plates with no prior specific mechanistic knowledge related to the proprietary platform process fragmentation, allowed to reveal trends and to qualitatively interpret them. Despite the rather high process variability and the loss of experimental conditions, 96-DWP can be considered to be a suitable tool for screening in the early phases of the research activities. Nevertheless, the trends were confirmed in ST and that system was successfully used to identify further substances affecting the level of fragmentation. Additional work is needed to obtain more thorough understanding of the processes involved in the fragmentation and to propose both feasible and efficient medium composition changes to limit the generation of LMW species. It is essential to produce stronger LMW changes in order to ascertain whether all observed LMW increases really were significant or whether the process variability in some cases was more important. For the time being, there are not sufficient data in the ST model available to estimate the process variability. The important inter-assay variability prevents a global statistical analysis of all experiments 
to quantify the process variability for LMW. A small number of replicates was chosen in each experiment as it was sufficient for other quality attributes including glycosylation. The observed fragmentation effects of the various cell culture supplements and the indication that Trx may play an important role, laid a foundation for future experiments.

\subsection{Conclusion}

Medium blending experiments in high-throughput fed-batch cell culture in 96-DWP revealed that the cysteine concentration in the supernatant correlated with the abundance of LMW species. It picked up relatively small changes despite large variability. The trends were confirmed in ST and the concentration ranges further extended, resulting in stronger responses. Cysteine promoted fragmentation in two different cell lines. $\mathrm{N}$-acetyl-cysteine, which enters more easily the cell, where it eventually disintegrates into cysteine, also increased fragmentation at lower concentrations. EDTA addition at the end of the culture did not affect the abundance of LMW species. DMSA, another chelating agent, however strongly increased fragmentation, which very likely was due to the antibody disulfide bond reduction by this related compound with mercaptoethanol. Ferric iron citrate slightly increased fragmentation, while copper and zinc did not show any effect. Finally, in the 96-DWP it was observed that fragmentation decreased with higher protein expression and consequently greater harvest product titer. 


\section{Chapter 7}

\section{Parallel Experimental Design and}

\section{Multivariate Analysis Provides Efficient Screening of Cell Culture Media Supplements to Improve Biosimilar Product Quality ${ }^{1}$}

\subsection{Introduction}

With the aim to reduce health care costs and to create wider access to biotherapeutics, particularly in emerging markets, the development of biosimilars has gained considerable interest ${ }^{4}$. As a copy drug, a biosimilar should be highly analytically similar to a commercial product and not exhibit clinically meaningful differences ${ }^{261,262}$. A biosimilar molecule and its respective innovator drug product cannot be considered bioequivalent as their manufacturing processes are not the same ${ }^{263,264}$. As of 2016, the European Medicines Agency (EMA) had approved 22 biosimilars including infliximab, the first biosimilar $\mathrm{mAb}^{265}$. The widespread progression of biosimilars due to the great potential to copy a selection of the 44 approved mAbs (end of 2014) ${ }^{247}$ has changed the landscape of process development. Aiming to match the quality profile of the targeted reference medicinal product (RMP) as closely as possible, product quality modulation evolved into a field of utmost importance to assure safe and efficient treatment of patients ${ }^{10}$.

1. Submitted, D. Brühlmann, M. Sokolov, A. Butté, M. Sauer, J. Hemberger, J. Souquet, H. Broly and M. Jordan, Parallel Experimental Design and Multivariate Analysis Provides Efficient Screening of Cell Culture Media Supplements to Improve Biosimilar Product Quality. This chapter is the result of a collaboration between Merck Biopharma and ETH Zurich. D. Brühlmann and M. Sokolov contributed equally. 
The cell line, the culture conditions and the medium composition affect recombinant protein quality attributes $7,17,45$. With respect to cell-line engineering, opting for media optimization considerably reduces development timelines and the complexity of product quality comparability considerations, which typically results from redevelopment of cell lines. High-throughput cell culture systems opened the door for testing many conditions simultaneously ${ }^{266-268}$. The technology developed for media optimization in 96-deepwell plates (96-DWP) fed-batch cultures $^{26,27,251}$ can also be applied for product quality modulation ${ }^{25,64}$. Good alignment of the glycan profiles between 96-DWP and shake tubes has been shown, and in the same study trends observed in high-throughput systems have been confirmed in lab-scale bioreactors ${ }^{28}$. In addition, by calling on rational experimental design strategies and the use of statistical tools, many quality attributes can be assessed simultaneously in media optimization ${ }^{269}$. This significantly improves the efficiency in process development by enabling to derive relevant knowledge in early process screening and to drive the process development to larger scales in a focused manner, resulting in reduced experimental efforts.

The goal of this work was to identify quality modulating compounds and interactions thereof. Nonetheless, in the identification process of seventeen potential candidates, technical limitations became evident for current DoE designs. With a large number of factors, the addition of multiple stock solutions likely entails non-negligible dilution effects of medium components. As a consequence, the accuracy of the model degrades. Furthermore, without prior evaluation of suitable concentration ranges, the risk to lose valuable information due to growth issues in wells containing suboptimal blends multiplies with increasing number of supplements ${ }^{27}$. The extent of the effect on product quality may also greatly vary from one supplement to another. Fundamental disparities may cover weaker modulation effects of other DoE factors. Thus, a new DoE approach was developed to overcome these limitations (figure 7.1). The streamlined and rational approach, intended as a first factor screening rather than a comprehensive interaction study, consisted in a parallel DoE method for seventeen compounds, putting an upper limit of five factors per DoE to minimize both dilution effects and the repercussions due to non-optimal conditions. Wherever possible, they were arranged in groups with factors producing similar glycosylation effects. Furthermore, prior univariate evaluation of suitable concentration ranges was performed. Multivariate analysis tools were used to evaluate the data of the parallel 5-factor experiments in 96-DWP to identify group winners. Subsequently, the interactions of the group winners were studied in a DoE in shake tubes, confirming the findings of the 96-DWP experiment, and the impact of a process parameter was assessed (effect of temperature). The data demonstrate that the combination of parallel group experiments of potential product quality modulating compounds with a multivariate selection process of the best performers enabled to rapidly improve medium supplementation concentration ranges to further approach, sequentially, the targeted glycosylation profile. 


\subsection{Materials and Methods}

\subsubsection{Inoculum Preparation}

A CHO-S derived clonal cell line expressing a human monoclonal IgG1 antibody was used in this study. The cells were first expanded in multiple passages in shake tubes or shake bottles in Merck proprietary chemically-defined medium containing methionine sulphoximine (MSX) for at least 14 days in a shaker incubator at $36.5{ }^{\circ} \mathrm{C}, 5 \% \mathrm{CO}_{2}, 80 \%$ humidity and $320 \mathrm{rpm}$ agitation (ISF1-X, Adolf Kühner, Birsfelden, Switzerland or Multitron Cell, Infors HT, Bottmingen, Switzerland)

\subsubsection{Cell Culture in 96-deepwell Plates and Experimental Design}

Potentially influential compounds were selected based on literature research ${ }^{203}$ and in-house experience to tune the product quality of the recombinant protein. Seventeen compounds were selected and added at three different levels $(-1,0$, and 1$)$ into the cell culture medium (table 1). The concentration ranges were defined as close to the optimum as the available prior knowledge enabled to, using concentrations mentioned in the literature (if available) and in-house experience with some of these compounds or similar ones. Rather than testing the interactions between the entire set of supplements simultaneously in one single experiment, it was decided to split the experiment into five separate, smaller sets of experiments. The more specific factors were grouped according to their biological mode of action. Each group also included two non-specific glycosylation modulation factors: manganese (Mn) and asparagine (Asn) ${ }^{25}$. Two groups comprised compounds favoring high mannose glycans (groups 1 and 2), group 3 included potential sialylation and charge variant modulators, fucosylation and galactosylation was the focus of group 4, while group 5 investigated the interactions between growth promoters and the non-specific compounds Mn and Asn (table 7.1). All group designs on 3 levels for 5 factors are shown in tables A.1 to A.5 (cf. appendix A). The five group experiments were conducted in parallel in two 96-DWP. The liquid handling for supplement enrichment of the individual wells and the sampling was performed by a robotic platform (Biomek FX, Beckman Coulter, Brea, CA). Exponentially growing cells were seeded into a shaking 96-DWP filled with Merck proprietary medium enriched with supplements in the absence of MSX at a viable cell density of $0.30 \times 10^{6}$ viable cells $/ \mathrm{mL}$. The plates were incubated with vented lids in a shaker incubator at $36.5{ }^{\circ} \mathrm{C}, 5 \% \mathrm{CO}_{2}, 90 \%$ humidity and $320 \mathrm{rpm}$ agitation (ISF1-X, Adolf Kühner, Birsfelden, Switzerland) for 14 days. $400 \mathrm{~g} / \mathrm{L}$ glucose solution, chemically-defined feed (CDF) containing over 30 components and alkaline amino acid solution (AAAS) were added on days 3, 5, 7, 10 and 12. Prior to each feeding and at the end of the culture (day 14), samples ( $\leq 40 \mu \mathrm{L}$ ) were drawn for cell counting (Guava easyCyte (Merck Lifesciences, Darmstadt, Germany) and product titer quantification (Octet ${ }^{\circledR}$, forteBIO, Menlo Park, CA). 


\subsubsection{Cell Culture in Shake Tubes and Experimental Design}

Keeping the process development workflow in mind, the selected group winners (section 7.3.2) were evaluated in TPP® TubeSpin bioreactor tubes (referred to shake tubes or $S T$ ) to test the validity at a larger scale with more control options. Three group winners were selected based on the methodology described in section 7.2.4, the concentration ranges were kept unchanged and a D-optimal quadratic design on three levels was chosen. Furthermore, as in future process development steps a temperature shift for productivity improvements may be considered, the process tunability at ST scale was exploited. For this purpose, an augmented D-optimal design at two levels including a temperature shift on day 5 of the culture was proposed (cf. table A.6 in appendix A).

Exponentially growing cells were seeded into ST tubes filled with Merck in-house medium in the absence of MSX at a viable cell density of $0.30 \times 10^{6}$ viable cells $/ \mathrm{mL}$. The tubes were incubated in a shaker incubator at $36.5^{\circ} \mathrm{C}, 5 \% \mathrm{CO}_{2}, 80 \%$ humidity and $320 \mathrm{rpm}$ agitation (ISF1-X, Adolf Kühner, Birsfelden, Switzerland) for 14 days. The temperature of ST experiments 1 to 15 (table A.6) were kept at $36.5^{\circ} \mathrm{C}$ during the entire fed-batch experiment, while tubes 16 to 22 underwent a temperature shift to $33^{\circ} \mathrm{C}$ on day 5 . CDF containing over 30 components and AAAS were added on days 3, 5, 7 and 10. The $400 \mathrm{~g} / \mathrm{L}$ glucose solution was added on these days and day 12 as well. Prior to each feeding and at the end of the culture (day 14), aliquots $(\leq 2.5 \mathrm{~mL}$ ) were taken for cell counting (Vi-Cell analyzer (Beckman Coulter, Brea, CA),

Table 7.1 - Group factor concentrations in medium prior to inoculation of 96-DWP.

\begin{tabular}{|c|c|c|c|c|c|}
\hline Group & Compound & Level -1 & Level 0 & Level 1 & Unit \\
\hline \multirow[t]{3}{*}{1} & Sucrose & 0 & 12.5 & 25.0 & $\mathrm{mM}$ \\
\hline & Raffinose & 0 & 12.5 & 25.0 & $\mathrm{mM}$ \\
\hline & Kifunensine & 0 & 5 & 10 & $\mu \mathrm{M}$ \\
\hline \multirow[t]{3}{*}{2} & Mannostatin & 0 & 5 & 10 & $\mu \mathrm{M}$ \\
\hline & Swainsonine & 0 & 5 & 10 & $\mu \mathrm{M}$ \\
\hline & Kestose & 0 & 2.5 & 5.0 & $\mathrm{mM}$ \\
\hline \multirow[t]{3}{*}{3} & EDTA & 0 & 25 & 50 & $\mu \mathrm{M}$ \\
\hline & Hydrocortisone & 0 & 10 & 30 & $\mu \mathrm{M}$ \\
\hline & ManNAc & 0 & 10 & 20 & $\mathrm{mM}$ \\
\hline \multirow[t]{3}{*}{4} & Galactose & 0 & 12.5 & 25.0 & $\mathrm{mM}$ \\
\hline & Uridine & 0 & 10 & 20 & $\mu \mathrm{M}$ \\
\hline & 2F-p-fucose & 0 & 30 & 60 & $\mu \mathrm{M}$ \\
\hline \multirow[t]{3}{*}{5} & Enhancer 1 & 0 & 5 & 10 & $\mu \mathrm{M}$ \\
\hline & Enhancer 2 & 0 & 15 & 30 & $\mu \mathrm{M}$ \\
\hline & Enhancer 3 & 0 & 50 & 100 & $\mu \mathrm{M}$ \\
\hline \multirow[t]{2}{*}{ All } & Manganese & 0 & 250 & 500 & $\mathrm{nM}$ \\
\hline & Asparagine $^{\mathrm{a}}$ & +0 & +2.5 & +5 & $\mathrm{mM}$ \\
\hline
\end{tabular}

a Asparagine is already present in the basal medium. 
extracellular metabolite profiling (Nova Bioprofile 100+, Nova Biomedical, Waltham, MA) and product titer determination (Biacore C instrument (GE Healthcare, Waukesha, WI).

\subsubsection{Product Quality Analysis}

At the end of each 96-DWP and ST fed-batch experiment (day 14), the supernatant was purified on small-scale affinity columns (PhytipsVR, PhyNexus, San Jose, CA). The eluates were analyzed by Ultra Performance Liquid Chromatography (UPLC)-2-amino-benzamide labelling technique on a $100 \mathrm{~mm}$ column (Waters Corporation, Milford, MA, USA) for glycosylation, by imaged capillary isoelectric focusing (iCE280 analyzer, ProteinSimple, Santa Clara CA) for charge variants, and by size-exclusion-high-performance-liquid chromatography (SE-HPLC, Waters, Milford, MA) for aggregation. In total, thirteen glycan species and five charge variant clusters (two acidic, one neutral, and two basic) were identified. Eluates of ST experiments were also analyzed by LabChip GXII protein assay (PerkinElmer, Waltham, MA) for low-molecularweight species content (LMW).

\subsubsection{Data Analysis}

The obtained results were visualized using box plots ${ }^{270}$. Then the multivariate analysis to select the process settings yielding a product quality as close as possible to targeted molecule was carried out according to Sokolov et al. ${ }^{269}$ in three characteristic steps. First, a PCA was performed on thirteen glycoforms (Y variables) and the number of relevant principal components (PCs) was quantified visually from the scree $\operatorname{plot}^{271-273}$. Subsequently, the quality of the originator molecule was projected onto the score space to determine the Mahalanobis distance $^{274}$ to this target (within the considered number of PCs) for each 96-DWP condition. The first two steps provided a basis for visual comparison of the different runs as well as for a quantitative evaluation of their performance with respect to the targeted originator molecule. The final step of the methodology built the connection of the process to the product, namely linking the media supplements $(\mathrm{Z})$ to the glycoforms $(\mathrm{Y})$ by a decision tree (DT). DTs are a systematic and automated tool providing a hierarchical order of (binary) decisions on the input variables (here media supplements) separating the output variable (here Mahalanobis distance to optimum) into maximally different groups. In order to avoid overfitting, the DT was cut back (pruned), using sevenfold cross-validation. Subsequently, this tree was analyzed with the goal to select the conditions, which tend to reduce the distance to the target, and to discard those tending to increase this distance. Finally, the results of the ST confirmation runs were used to validate the findings of above multivariate factor selection analysis based on 96-DWP experiments and to provide additional process understanding to define a basis for further process development. 


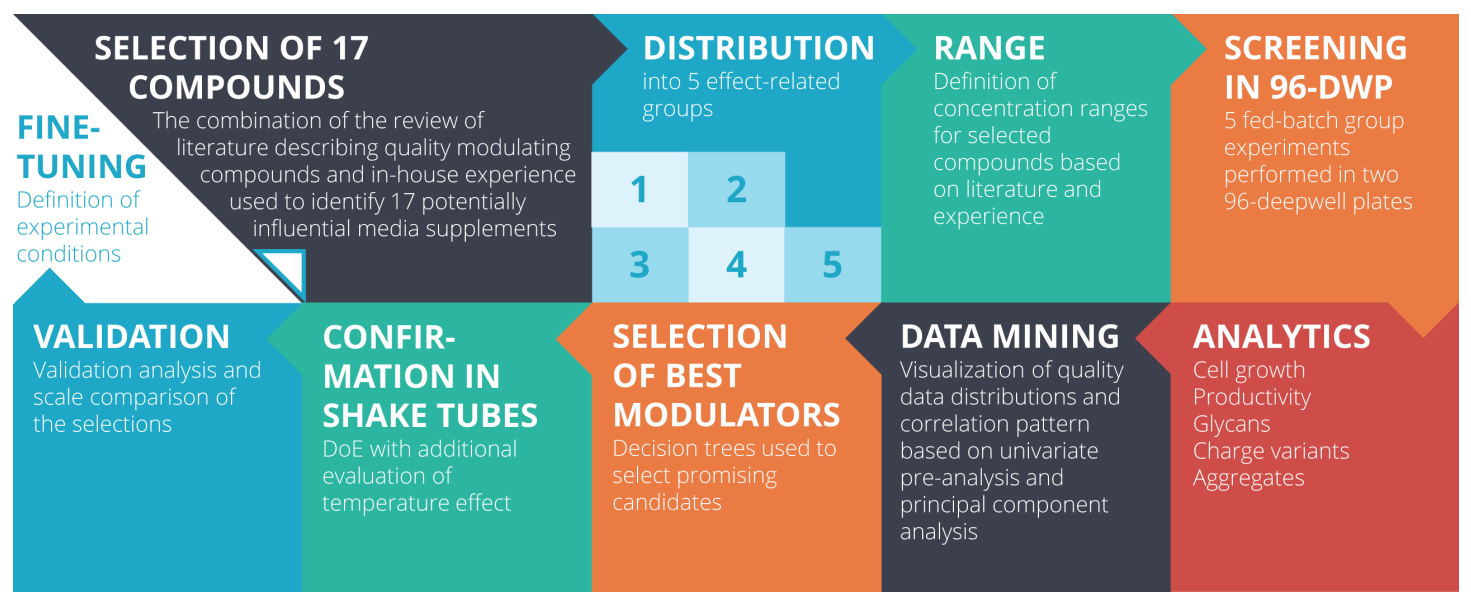

Figure 7.1 - Sequential design of experiments method using characteristic compound groups and multivariate selection of best quality modulating compounds.

\subsection{Results and Discussion}

\subsubsection{Cultures in 96-deepwell Plates}

The seventeen medium supplements of the five effect-related groups produced important glycosylation pattern changes. Each quality attribute class will be discussed separately hereafter.

\section{High Mannose Species}

Figure 7.2 shows that experimental group 2 entailed the strongest response on the abundance of high mannose species (Man4 to Man7). In particular, the presence of swainsonine yielded a peak at $98 \%$, while in the absence of this strong modulator the glycan processing was highly favored. Consequently, bountiful complex glycans resulted and high mannose species dropped to $1.4 \%$. Group 1 featured the second strongest effect with respect to the oligosaccharide levels, which started at $1.4 \%$ and reached $6.6 \%$ at the most. Thereby, many conditions of group 1 did not grow as a consequence of the high kifunensine concentrations in the medium at levels 0 and 1 . Hence, dividing the 17 compounds into five groups proved to be of great benefit to reduce such information loss to group 1 only. The high mannose levels in groups 3 to 5 were comparable to the control samples $(\mathrm{n}=8)$.

\section{Afucosylated Glycans}

The supplementation of 2F-peracetyl-fucose in group 4 was found to affect the level of afucosylated glycans (A0, A1 and A2). While the levels in groups 1 to 3 and 5 were comparable to the controls, group 4 exhibited a strong increase of afucosylated forms from 1.9 to $89.3 \%$. All 
five groups induced considerably higher variations of the FA2 glycan levels compared to the controls. FA2 varied between 0.7 and $86.6 \%$ (in group 2) and between 0.9 and $85 \%$ (in group 4).

\section{Galactosylation and Sialylation}

Likewise, groups 2 and 4 were characterized by the strongest response of the galactosylated species FA2G1 and FA2G2. However, for the galactosylated species the variations in groups 2 and 4 were only slightly higher than in the remaining groups. The strong increase of both high mannose in group 2 and afucosylated forms in group 4 accounted for the immense variation of the FA2. To a lesser extent, this change was due to increased galactosylation as the smaller FA2G1 and FA2G2 differences revealed. It shows that FA2 was strongly linked to its glycosylation precursors and successors in the glycosylation network. All media supplements induced small sialylation changes, which were, after all, significantly greater than the variation of the control cultures. Overall, manganese very likely was the supplement displaying the strongest galactosylation tuning effect-in agreement with the literature ${ }^{122}$-and possibly yielded similar ranges of galactosylation in all five groups.

\section{Charge Variants and Aggregation}

The effect of the supplements on the charge variants (clusters 1 to 5 ) as well as the aggregates was assessed also (figure 7.3). The clusters 1 to 4 in the groups 1 and 2 showed variations larger than the control runs, while for cluster 5 no group featured a clear variation compared to the controls. A decrease in aggregate levels during cell culture to circumvent undesired immunogenicity is an important objective ${ }^{243,275}$. Each group increased the range of protein aggregation compared to the controls. Group 3 stood out, due to the presence of hydrocortisone, demonstrating its capability to decrease protein aggregation. It is not clear whether this hormone influenced protein expression or stabilized the secreted antibody in the supernatant.

\subsubsection{Identification of the Best Glycosylation Modulators}

Considering the fact that glycosylation has a major effect on pharmacokinetics (PK) and protein physicochemical characteristics ${ }^{14}$, the study focused on the glycan tuning. Progressing in the rational design of experiment endeavor, the goal was to identify, among the entire array of seventeen compounds, those allowing to get as close as possible to the specifications for biosimilarity (referred to optimum in the subsequent charts). The 96-DWP PCA score, loading and scree plots are all provided in the supplementary material (figures A.1 to A.3 in appendix A). Figure 7.4A shows the score plot for a joint PCA on the 96-DWP and ST data, which shall also be used for later scale comparison. At this stage, one can point out that the majority of the 96-DWP experiments form a diagonal cluster far from the projected glycosylation optimum (figure A.1 in appendix A), while a cluster of group 4 experiments wound up even farther 
A

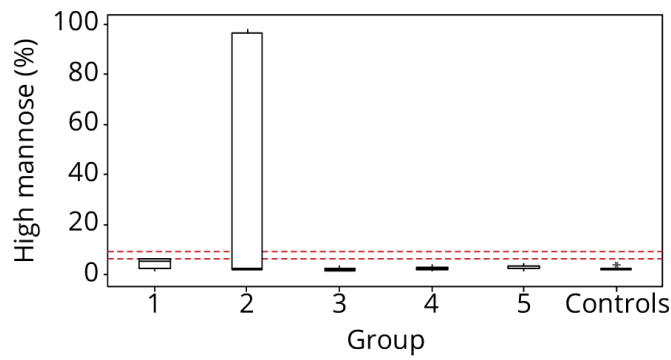

C

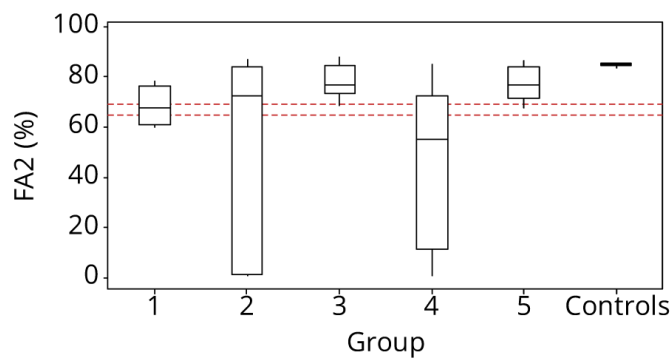

$\mathrm{E}$

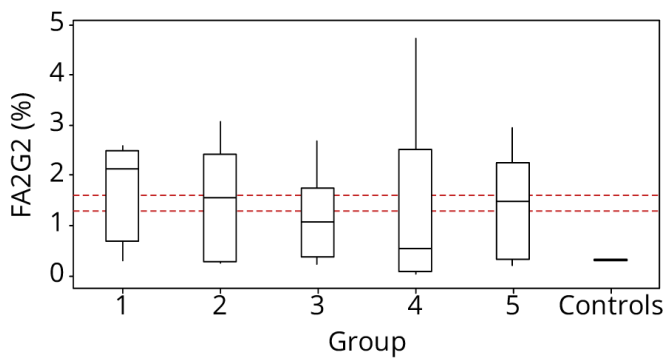

B

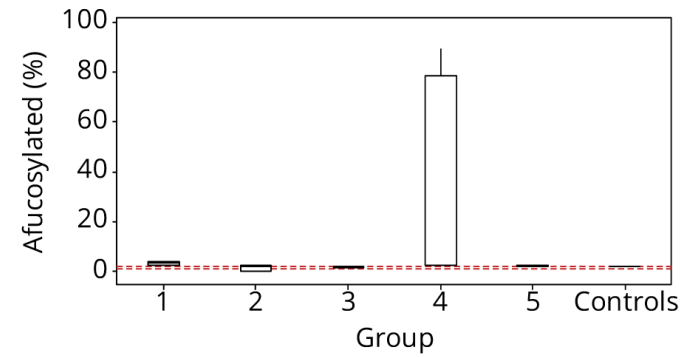

$\mathrm{D}$

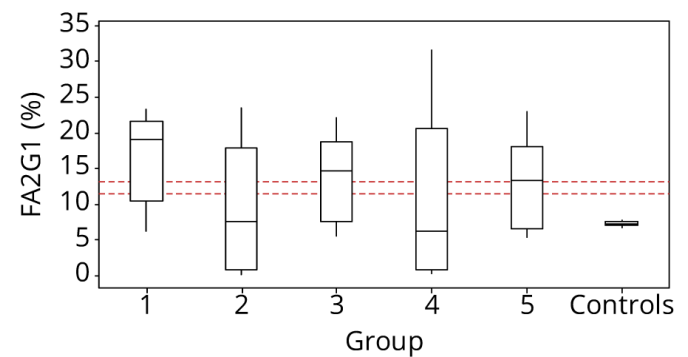

$\mathrm{F}$

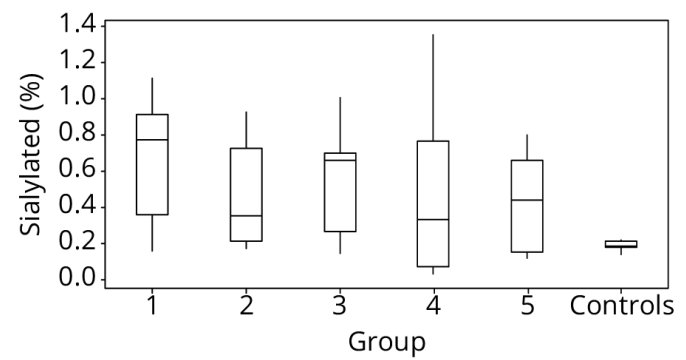

Figure 7.2 - Boxplots of glycan modulation ranges. The group independent control samples were conducted in 8 replicates: 4 on each 96-DWP plate. The dashed lines mark the respective specification ranges, where applicable. (A) High mannose glycan modulation ranges in each group (1-5). Man4 to Man7 were detected and summed up. (B) Modulation ranges of afucosylated species including A0, Al and A2 in the five groups. (C-E) The three charts present agalactosylated species (FA2), the sum of monogalactosylated species FA2[3]G1 and FA2[6]G1 as well as the abundance of digalactosylated glycan (FA2G2). (F) The sialylated forms FA2G2S1, FA2G2S2 and FA2G2S1(NGNA) were grouped in one single chart. 
A

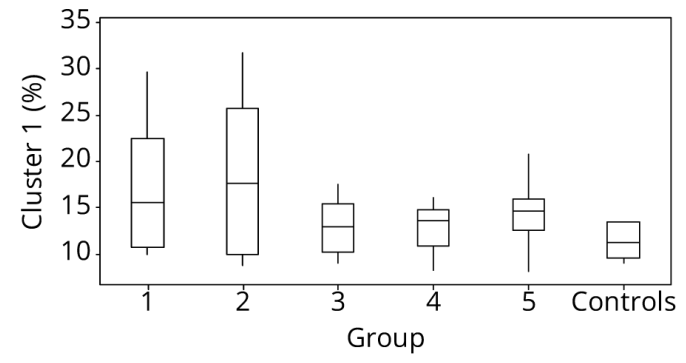

C

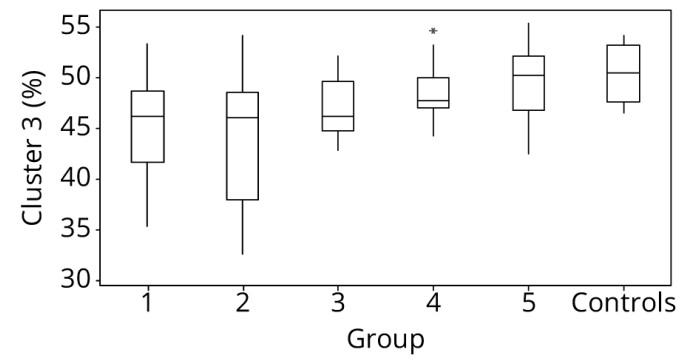

$\mathrm{E}$

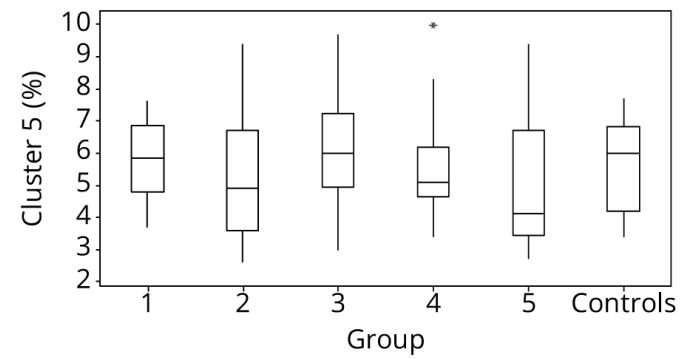

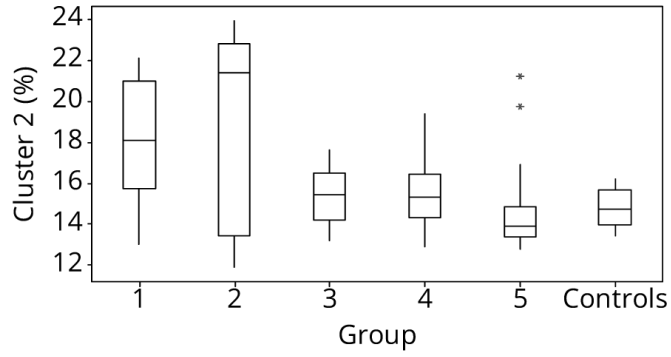

D

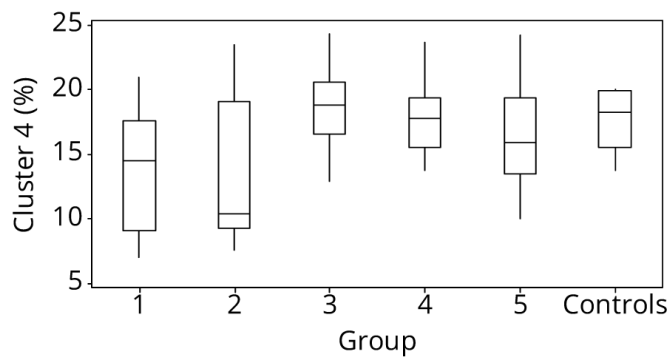

$\mathrm{F}$

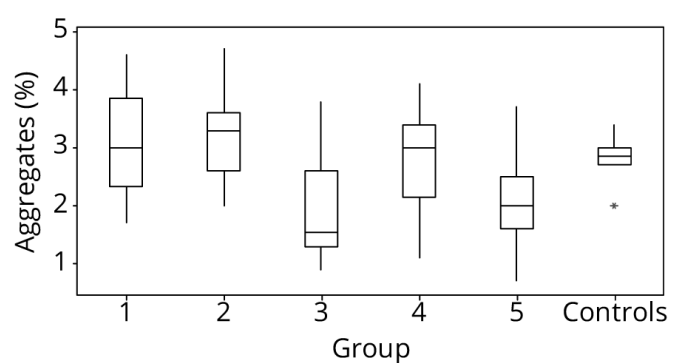

Figure 7.3 - Boxplots showing the range of charge variants and aggregation levels. The group independent control samples were conducted in 8 replicates: 4 on each 96-DWP plate. (A-E) The charge variants were grouped into 5 clusters: acidic (1-2), neutral (3), basic (4-5). The charts show the corresponding ranges within the five groups. (F) The aggregate ranges of each group are displayed. 
from the optimum at the right bottom corner. Those experiments were supplemented with 2F-peracetyl-fucose. The scree plot (figure A.3 in appendix A) shows a characteristic elbow at four PCs, indicating that the three PCs are likely to represent genuine biological effects. The first three PCs account for $76 \%$ of the glycan variance and thus indicate strong correlation of the glycans. The loading plot (figure A.2 in appendix A) shows the correlation structure of thirteen glycoforms. Hence, an analysis in reduced dimensions is feasible.
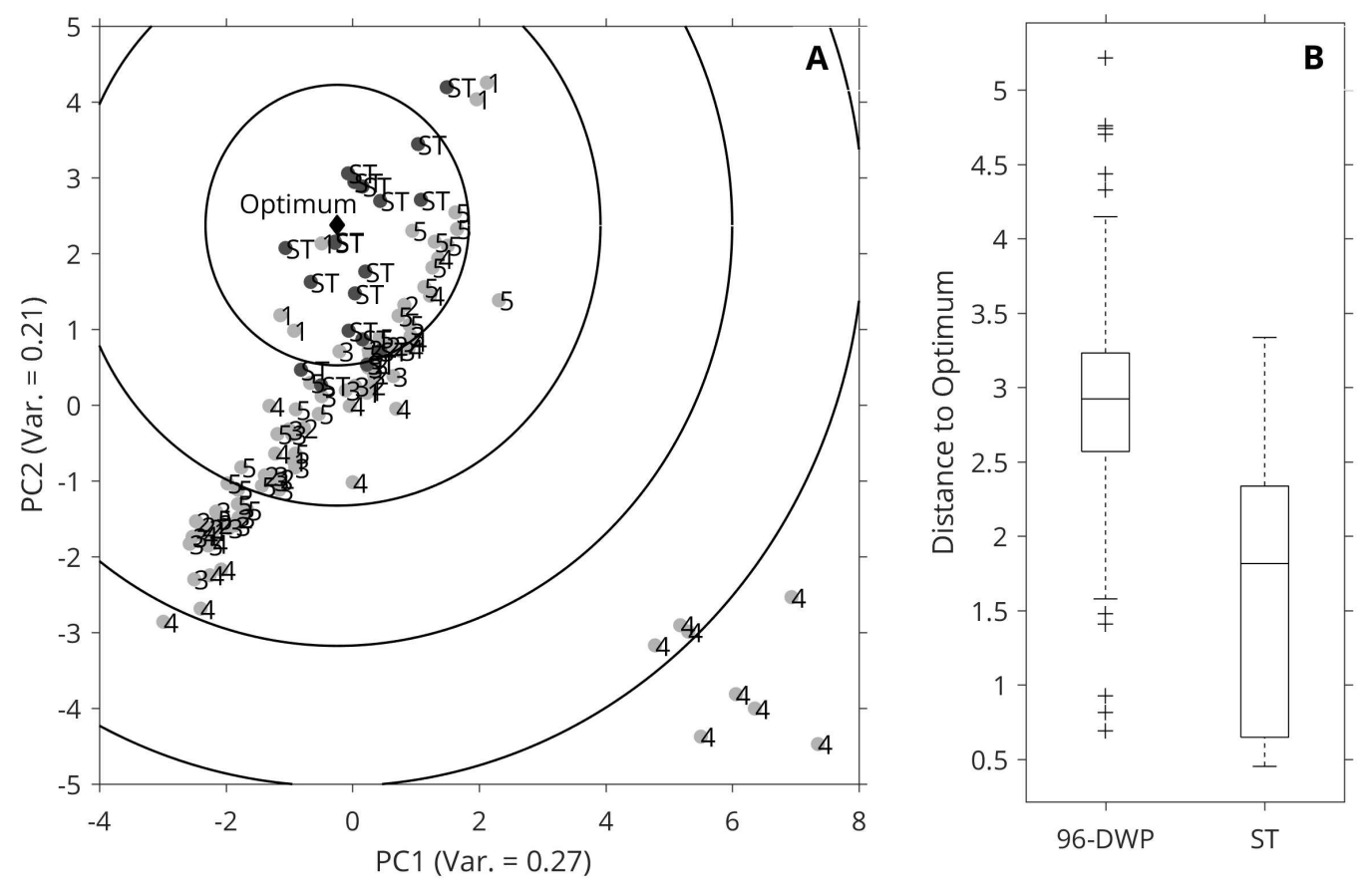

Figure 7.4 - (A) Score plot for joint PCA of 96-DWP experiments (light gray) and of ST experiments (deep gray) with projected optimum. The first two PCs are shown explaining almost $50 \%$ of the total variance. Ellipses show equidistant conditions according to Mahalanobis distance (1 to 4 distance units in the plain of the first two PCs). (B) Boxplots showing distance to optimum for 96-DWP and ST experiments based on first 3 PCs. PC3 explains additional $16 \%$ of variance. The plus symbols mark outliers.

The second analysis step was the quantification of the deviation from the optimum by the Mahalanobis distance. Figure 7.5 specifies the observations from the score plot, demonstrating that with increasing addition of 2F-peracetyl-fucose, product quality is dragged away from the optimum (median equal to 6 distance units at the highest concentration). Moreover, it was demonstrated that with increasing raffinose concentration the experimental conditions approached the optimum to a great extent, entailing a decrease of the median from about 4 to 1 Mahalanobis distance units. Enhancer 2 supplementation caused a slight decrease (median falling below 4), whereas the median of manganese remained unchanged at all concentration levels.

The analysis in figure 7.5 shows simple trends for the supplements. In order to select the 

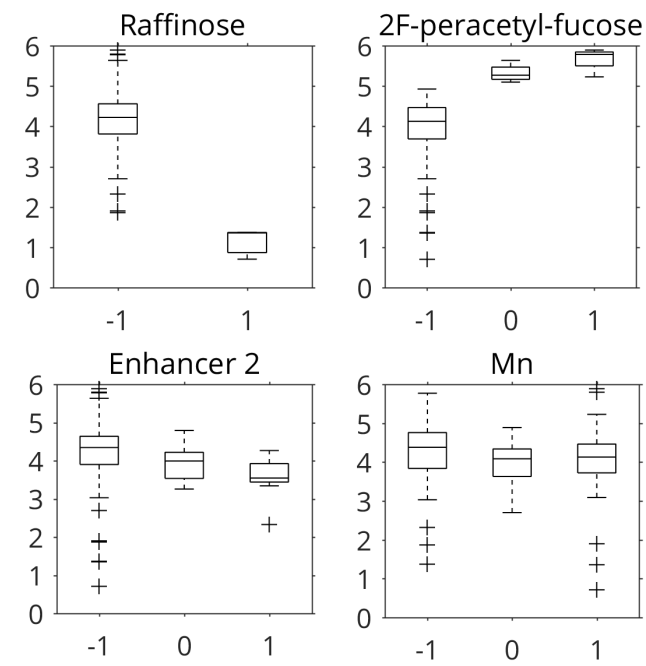

Figure 7.5 - Boxplots showing Mahalanobis distance to optimum in function of the concentration level of four compounds in 96-DWP experiments. The plus symbols mark outliers.

relevant glycosylation modulators among the seventeen media supplements at optimal levels as well as to rank their relevance a cross-validated (pruned) decisions tree (DT) was used. The DT shows the relevant decisions on the supplements, their levels and how these are sequentially impacting the distance from the optimum (figure 7.6). Based on the analysis of the DT, one can conclude that the largest effect can be obtained by increasing raffinose concentration, which allows to significantly approach the optimum. Secondly, 2F-peracetylfucose supplementation should be avoided due to its strong inhibition of fucosylation, which is not beneficial in this case. As a result of the presence of this fucose analog, the average distance to the optimum climbed up to 5.5. Sucrose addition also decreased the distance. Nonetheless, aiming to select one winner per effect-related group this compound was not further considered due to its similar behavior to raffinose. For group 5 experiments, the tree also recommended to use enhancers 1 and 2. Likewise, enhancer 2 (with the slightly stronger effect) was retained only. Last, galactose supplementation yielded a slightly favorable effect, decreasing the average distance by about 0.9 units. Even though this difference was small, galactose was kept as it favored higher titers (data not shown). Manganese exhibited both positive and negative effects on the various glycoforms (data not shown), so that those opposing trends made this compound never appear in the DT. The intention was to focus the selection process singly on the supplements featuring a clear capability of improving the entire product quality towards the optimum and to spread those selections among different DoE groups, so to have a media supplement toolkit affecting different parts of the glycosylation pattern. The selection of raffinose rather than sucrose and enhancer 2 rather than 1 draws the attention to the importance to rely at once on statistical analysis and prior experimental data as well as scientific rationales in the selection process ${ }^{276}$. The third selected group winner was galactose. It is important to highlight that those selections are highly dependent on the characteristics of the biotarget and the concentrations, at which the various species were 
analyzed. Therefore, the relevant effects of sucrose, enhancer 1 and 2F-peracetyl-fucose (at the investigated concentrations) shall be remembered in the framework of effective glycosylation modulators, while for the remaining components, a modification of the concentration range could be considered in further experiments.,

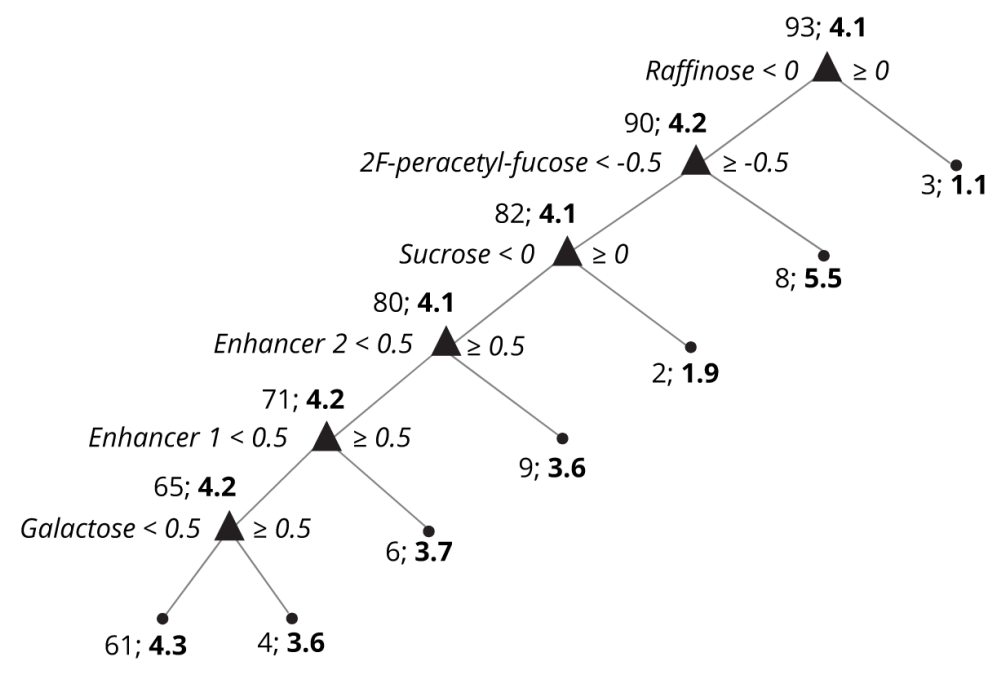

Figure 7.6 - Pruned decision tree for selection of best glycosylation modulators. At each node the number of observations (regular), and the average distance to the target (bold) is provided. The concentration level of the decision variable is shown in italic.

\subsubsection{Verification and Extension in Shake Tubes}

Addition of raffinose, galactose and enhancer 2, as well as the temperature shift on day 5 in ST (table A.6 in appendix A) modulated the product quality in the experiments. According to figure 7.7, the HM glycans resided between 1.5 and $4.7 \%$ in the absence of a temperature downshift. The temperature shift led to an overall increase, amounting to $2.3-5.6 \%$ of HM. Mild hypothermia has been shown to impact galactosylation depending on media composition, cell line and protein type ${ }^{277-279}$. As expected based upon biological knowledge, the media supplements weakly influenced the abundance of afucosylated species. The design produced an important interval of galactosylation level. At $36.5^{\circ} \mathrm{C}$, the fucosylated species (FA2) ranged between 66.6 and $83.8 \%$, while the galactosylated species FA2G1 and FA2G2 amounted to 9.4-18.4\% and $0.4-1.3 \%$, respectively. At $33^{\circ} \mathrm{C}$, the level of galactosylation increased as the lower FA2 box highlights, and consequently, a rise of the mono- and di-galactosylated boxes resulted. FA2 varied between 59.9 and $78.0 \%$, FA2G1 between 13.5 and $24.5 \%$ and FA2G2 between 0.8 and $2.1 \%$.

Second, the glycopattern obtained in ST shall be compared to the 96-DWP product quality results. The PCA score plot (figure 7.4A) displays that several experiments in ST greatly approached the optimum and that most of the ST experiments are located closer or equally close 
A
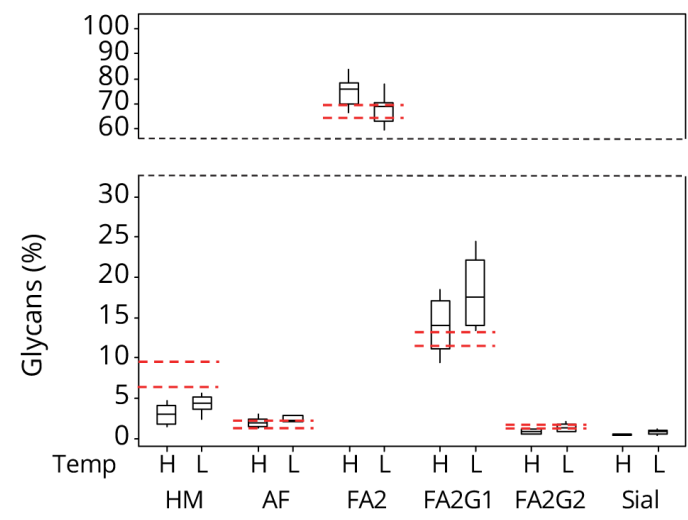

B
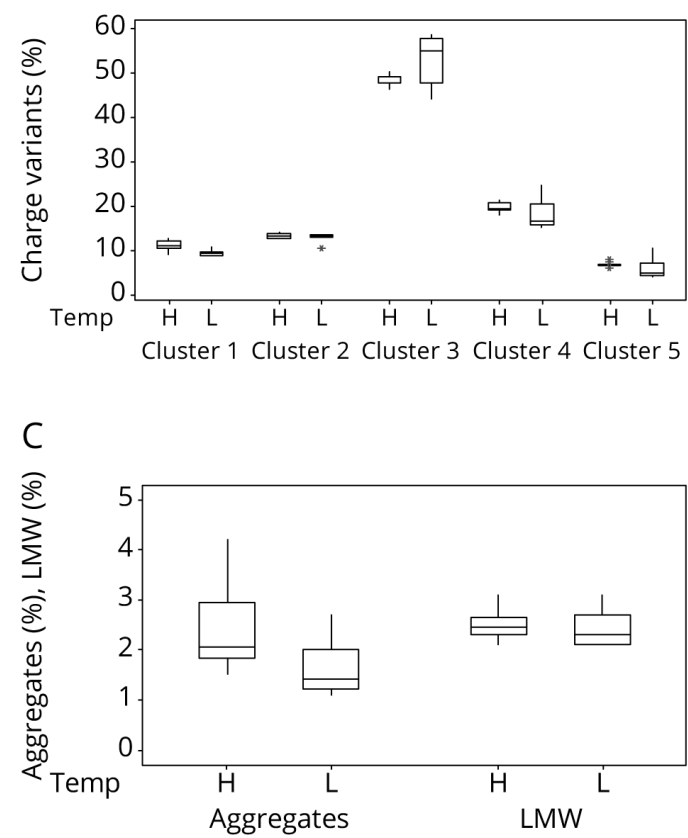

Figure 7.7 - (A) Boxplots of glycan modulation ranges obtained with raffinose, galactose and enhancer 2 in ST bioreactor tubes at $36.5^{\circ} \mathrm{C}(\mathrm{H})$ and when lowering the temperature to $33^{\circ} \mathrm{C}$ on culture day 5 (L). The dashed lines represent the specification ranges, where applicable. (B) Boxplots of the charge variants: acidic (clusters $1 \& 2$, neutral (cluster 3), basic (clusters 4 \& 5). (C) Boxplots of aggregates and low molecular species (LMW). 
to the optimum compared to the 96-DWP experiments. The positive effects of the selected glycosylation modulators can be further recognized in the comparison of the Mahalanobis distance distributions (calculated for first three PCs) shown in figure 7.4B. The median of the distance from the target decreased from about 3 units in 96 DWP to below 2 in the ST experiments. One can highlight that, in fact, $25 \%$ of the experiments in ST feature a distance smaller than 1 unit with the smallest value in the region of 0.5 . Moreover, $75 \%$ of ST conditions performed as well or better than the best $25 \%$ in 96 -DWP. These results confirm that modulator selection was beneficial for targeted process development. It is noticeable that even the ST experiments with four factors still yielded large glycan variability, which is of great value to further fine-tune the quality in a subsequent optimization round. Figure 7.8 visualizes the effect of those four factors on the distance from the target, depicting the presence of raffinose both at levels 0 and 1 was beneficial (median of the distance to the target at about 0.8 ). Also, the addition of enhancer 2 resulted in both lower medians and boxes. In particular, at level 1 the median significantly decreased to 0.5 . Those two trends parallel the downward trends visible in the 96-DWPs (figure 7.8). The temperature shift at culture day 5 led to a considerable decrease of the median from about 2 to 0.7 , as well as the box. With the exception of an outlier, all conditions that underwent a temperature decrease to $33^{\circ} \mathrm{C}$ on day 5 featured a distance between 0.5 and 1.5 units from the optimum. Thus, lowering the temperature proved to be beneficial in reaching the optimum. Unlike the other factors, the potential benefit of galactose could not be confirmed. Galactose did not feature a clear downward trend for the ST experiments. This is not unexpected as it appeared as the last (least relevant) decision criterion in the DT (figure 7.6). More importantly, the addition of galactose proved to yield higher titers in the ST experiments, justifying its inclusion as media supplement (data not shown). Taken together, the overall recommendations from the analysis of the 96-DWP experiment were confirmed in ST, showing the great advantage of performing early screening experiments in a high-throughput system. It was possible to reproduce the observed trends in ST, which is in line with the data published earlier, using a different cell $\operatorname{line}^{28}$. Even though some of the conclusions of the selection process were based on only a few experiments due to the loss of some conditions (e.g. three experiments with added raffinose), a critical statistical analysis (targeted to find general trends using cross-validation) combining all the results of the group experiments proved to be powerful. The improvement obtained in the second experiment can also be seen in table 7.2. The percentage of the experimental conditions within specification increased for most glycan species. The highest increase was obtained for A1 ( $74 \%$ vs. $27 \%$ ) and FA2 (32\% vs. $11 \%$ ). Although raffinose addition favored high mannose species, with the exception of Man7 (21\% vs. $3 \%$ ), none of the ST experiments fell within the specification range of these glycans. In fact, one experimental condition (with all three group winners added and at low temperature) resulted in five of the ten glycans at sufficiently large absolute concentrations being in-specification. It can be used as the center point for a subsequent experimental series. For further improvement, it is suggested to perform a univariate analysis to enrich the so far derived effective media supplement toolkit by specific drivers among the array of 17 components such as manganese for further fine-tuning of the glycans still out-of-specification. This specific glycan modeling can be refined with further 
process information (such as viable cell density, productivity) to increase the mechanistic knowledge by integrating further important characteristics besides the media composition in the framework of glycosylation modulation ${ }^{122,280-282}$. At later stages of process development, such additional (dynamic) characteristics are likely to play a key role in building predictive process models.
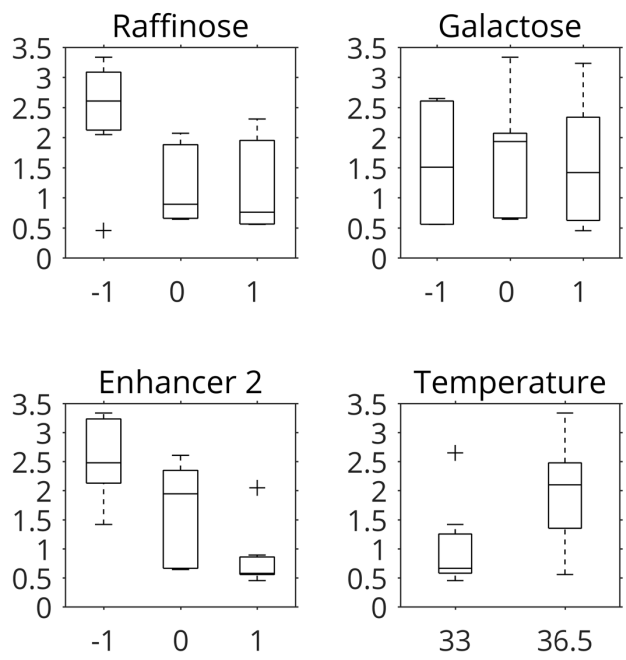

Figure 7.8 - Boxplots showing Mahalanobis distance to optimum in function of the concentration levels of the compounds and the culture temperature from day 5 of ST experiments. Plus symbols mark outliers.

Despite the fact that the ST experiments focused on the glycan tuning to reach the glycosylation optimum, the ramifications on charge variants, aggregation and low-molecular-weight species (LMW) were assessed too. The temperature decrease strongly extended the ranges of the neutral and basic clusters. Furthermore, the acidic cluster 1 slightly decreased. The effect of the culture temperature on charge variants has been reported previously, correlating a decrease of acidic forms and deamidation of IgGs with decreasing temperature ${ }^{283,284}$. The temperature shift produced lower aggregation, and potentially simplifies the required downstream activities. The low-molecular-species remained in a tight interval in all tested conditions.

\subsection{Conclusions}

Grouping 17 potential quality modulating medium supplements into five parallel experiments in 96-DWP produced wide glycosylation ranges, and in particular, a great modulation potential for afucosylated and galactosylated species. The combination of rational high-throughput optimization and multivariate analysis proved to be a powerful approach. Principal component analysis for visual comparison of the different runs, the determination of the Mahalanobis distance for a quantitative evaluation of their performance with respect to the optimum as well as the subsequent selection process following a hierarchical order of decisions on process 
Table 7.2 - Comparison of fulfillment of the specifications for biosimilarity of experiments in 96-DWP and ST. The structure of each glycan is shown: $\mathrm{N}$-acetylglucosamine (blue square), mannose (green circle), fucose (red triangle), galactose (purple circle). For each cell culture system the percentage of experiments reaching the optimum for the corresponding glycan are presented.

\begin{tabular}{|c|c|c|c|}
\hline Glycoform & Structure & DWP: within spec & ST: within spec \\
\hline A ${ }^{*}$ & & $0 \%$, far & $0 \%$, far \\
\hline Al & $=\{$ & $27 \%$ & $74 \%$ \\
\hline A2 & & $2 \%$ & $11 \%$ \\
\hline FA1 & - & $34 \%$ & $42 \%$ \\
\hline FA2 & & $11 \%$ & $32 \%$ \\
\hline FA2[6]G1 & & $11 \%$ & $16 \%$ \\
\hline FA2[3]G1 & & $12 \%$ & $21 \%$ \\
\hline FA2G2 & & $6 \%$ & $5 \%$ \\
\hline Hybrid-F ${ }^{*}$ & & $3 \%$ & $0 \%$, far \\
\hline $\operatorname{Man} 4^{*}$ & & $0 \%$, far & $0 \%$, far \\
\hline Man5 & & $0 \%$, close & $0 \%$, close \\
\hline Man6 & & $2 \%$ & $0 \%$, close \\
\hline Man7 & & $3 \%$ & $21 \%$ \\
\hline
\end{tabular}

\footnotetext{
* These species featured very low absolute concentrations close to their detection limit.
} 
variables using a decision tree enabled to select the best performing supplements in a systematic and automated way. The verification experiments in ST not only validated the conclusions of the selection process, but the experimental conditions significantly approached the optimum: $75 \%$ of ST conditions performed equally well or better than the best $25 \%$ in 96 -DWP. Furthermore, it provided the opportunity to include the culture temperature, an important process parameter, at this early stage, which paved the way for further glycosylation improvements and reduced aggregate formation. The presented method limited detrimental impacts of sub-optimal conditions as a result of unfavorable concentration ranges and masking effects by compounds with much stronger responses. Despite the loss of many cultures in group 1, meaningful 96-DWP results were obtained and confirmed in ST. The great added value of this method arises from the reduction of the high complexity due to the handling of two distinct fed-batch culture systems, multiple product quality attributes and consequently large data sets. In only two rounds of experiments, the glycosylation pattern of the selected experimental conditions substantially approached the optimum, which may entail significant experimental time savings (up to 3 to 6 months) and reduction of costly quality analysis testing (>50\%), and hence, huge cost-savings. The presented effective and target-oriented method significantly reduces the complexity of the initial screening procedure to select important glycosylation modulators. In the framework of cell culture process development, it provides a first process knowledge basis, which shall be further refined and optimized at the subsequent development stages usually performed at larger experimental scale.

\subsection{Acknowledgement}

We acknowledge the colleagues from Merck Biotech Process Sciences Analytics for their valuable support and the Department of Chemistry and Applied Biosciences, Institute for Chemical and Bioengineering of ETH Zurich for conducting the multivariate analysis. We thank in particular Michael Sokolov for providing figures 7.4, 7.5, 7.6 and 7.8. 



\section{Chapter 8}

\section{Linking Metabolomic Profiling with Glycosylation}

\subsection{Introduction}

Once the glycosylation profile and other critical post-translational modifications of the recombinant protein have been successfully adjusted in the development stage, the biotechnology industry must continually deliver drugs within specification to supply the market with consistent quality. The biochemical mechanisms of $\mathrm{N}$-glycosylation in the endoplasmic reticulum (ER) and the Golgi apparatus are highly complex and involve many different processes, enzymes, substrates and cofactors ${ }^{24}$. Furthermore, the metabolism of the host cell is linked with the glycosylation pattern of the recombinant protein, and hence, metabolic control may come into play to influence $\mathrm{N}$-glycan processing ${ }^{106,123,135}$. Metabolomic analysis of fed-batch cultures revealed increasing ornithine levels coincided with higher high mannose glycan levels ${ }^{285}$. In continuous $\mathrm{CHO}$ cell culture producing interferon- $\gamma$, site glycosylation occupancy was linked with the intracellular UDP-N-acetylgalactosamine concentration ${ }^{79}$. Ammonium plays an active part in the regulation of the $\mathrm{pH}$ of acidic intracellular compartments of $\mathrm{CHO}$ expressing recombinant immunoadhesin tumor necrosis factor-IgG (TNFR-IgG), including trans-Golgi and it affects intracellular nucleotide sugar pools, especially UDP-N-acetylglucosamine (UDP-GlcNAc) and UDP-N-acetylgalactosamine ${ }^{237}$. The availability of nucleotide sugars in the lumen of the Golgi apparatus also depends on the transport, as shown in Trypanosoma brucei, where the inhibition of transporters modified surface glycosylation ${ }^{286}$. Increasing levels of glycosylation precursors, as result of cell culture medium supplementation for instance, alters the glycan distribution ${ }^{124}$. Moreover, the most abundant cation in vertebrates, calcium $\left(\mathrm{Ca}^{2+}\right)$, regulates the intra-Golgi membrane transport ${ }^{287}$. Metal ion dependences have also been observed in other contexts. Various metal ions promote galactosyltransferase (GalT) activity by a synergistic effect of the metal and UDP-galactose (UDP-Gal), forming a bridge complex with the enzyme ${ }^{288}$. A synthetic model of the intra-Golgi transport 
highlights the importance of the three processes that equitably partake in membrane traffic: cisternal maturation, lateral diffusion, and a mostly retrograde vesicle-mediated transport ${ }^{289}$. All these observations depict the complexity and huge diversity of the various processes involved in the glycosylation pathway.

Metabolic flux studies and the corresponding models enable better understanding of glycosylation ${ }^{14}$. Notably, the visualization of the glycan distribution eases the study of reaction paths contingent on the various physiological or culture conditions ${ }^{290}$. Since N-glycan maturation in the endoplasmic reticulum and the Golgi consists in dynamic and non-template-driven processes, it creates a high degree of structural diversity, molecular dynamics simulation and glycan flux analysis may be considered ${ }^{291}$. High-throughput profiling of nucleotides and nucleotide sugars provided an insight into the effect of media additives on the intracellular nucleotides and nucleotide sugars, and thus, it was observed that the impact of elevated UDPGlcNAc and GDP-fucose (GDP-Fuc) levels on the final glycosylation patterns was marginal, while the UDP-Gal synthesis seemed to be limiting, showing the link between precursor availability and glycosylation maturation ${ }^{292}$. Despite the numerous factors that influence the enzymatic machinery in the secretory pathway, a dynamic mechanistic model successfully predicted time evolution of mAb glycosylation profiles during a fed-batch process ${ }^{293,294}$. Likewise, a dynamic mathematical model based on cisternal maturation by simplifying the Golgi apparatus to a plug flow reactor and by including recycling of Golgi-resident proteins not only describes glycosylation profiles of mAbs but also the result of fucosyltransferase gene silencing and cytosolic nucleotide sugar donors depletion with respect to the glycosylation fingerprint ${ }^{295}$. That work, as the authors highlighted, coupled the cellular metabolism with glycosylation. As an alternative to kinetic models, it was proposed to describe glycosylation as a stochastic process by means of methods from Markov chain theory and flux balance analysis, which was employed to predict and experimentally validate glycan patters of EPO, IgG and the endogenous secretome subsequent knock-out of glycosyltransferase in distinct $\mathrm{CHO}$ cell lines ${ }^{296}$.

With the aim to link metabolomic data with the glycosylation distribution of the expressed antibody, non-targeted metabolomic profiling of a monoclonal antibody expressing $\mathrm{CHO}$ cell line cultured in four distinct process formats was performed. As a starting point, univariate analysis of intracellular and extra-cellular profiles of various metabolites was used to reveal substantial differences in a variety of pathways. Moreover, the effect of the medium and feed composition on the time evolution of the glycosylation profiles as well as the ratio between fucosylated and galactosylated subspecies was studied. Intracellular levels of nucleotide sugars were contrasted with the observed differences of the respective glycan distributions. Driven by the limitations of univariate analysis of the huge metabolomic data set, the use of multivariate analysis (MVA), namely principal component analysis, was evaluated to reduce the complexity and to draw meaningful conclusions. These tools allowed to pinpoint the intracellular metabolites that correlate with time-dependent glycan profiling data. They provide a basis for a more comprehensive, and importantly, pathway-focussed data analysis. Finally, this chapter presents a partial-least-square multivariate model to predict the glycosylation pattern. The 
observation model built with three of the four processes is capable to predict the glycosylation profile of an external data set, namely the fourth process, of a specific culture day based on the extracellular metabolite levels.

\subsection{Materials and Methods}

\subsubsection{Inoculum Preparation}

A CHO-S derived clonal cell line expressing a human monoclonal IgG1 antibody was used in the frame of this study. Cells were first expanded in multiple passages in shake tubes or shake bottles in proprietary medium containing methionine sulfoximine (MSX) for at least 14 days in a shaker incubator at $36.5^{\circ} \mathrm{C}, 5 \% \mathrm{CO}_{2}, 80 \%$ humidity and $320 \mathrm{rpm}$ agitation (ISF1-X, Adolf Kühner, Birsfelden, Switzerland or Multitron, Infors HT, Bottmingen, Switzerland).

\subsubsection{Cell Culture in 3.5-L Bioreactors}

This study was focusing on the process performance, metabolite profiling and glycosylation pattern of four different bioreactor processes formats (Process A, B, C, D) in parallel, using the same cell line. The processes were performed with different media and main feed compositions. The media of processes A, B and D exhibited distinct levels of asparagine, while the medium of process $\mathrm{C}$ was characterized by changes of the levels of various components according to table 8.1. Exponentially growing cells were seeded at $0.30 \times 10^{6}$ viable cells $/ \mathrm{mL}$ in 3.5-L bioreactors (Biostat B, Sartorius, Göttingen, Germany; final volume: 3.0 L) filled with proprietary medium in the absence of MSX. The chemically-defined feed (CD-feed) supplemented with one or several compounds including trace elements (TE), manganese (Mn) and galactose (Gal) according to table 8.2 and the alkaline amino acid solution were added on days $3,5,7$ and 10 . Glucose was fed daily from day 3 to the end of the culture. For process $C$, an additional asparagine feed was added on days 3 and 5 .

Table 8.1 - Media supplementation of the four 3.5-L bioreactor fed-batch processes.

\begin{tabular}{ccc}
\hline Process & Basal Medium & Asn (-) \\
\hline A & Medium 1 & $1 \times$ \\
B & Medium 1 & $2.6 \times$ \\
C & Medium 2 & $1.8 \times$ \\
D & Medium 1 & $2.6 \times$ \\
\hline
\end{tabular}




\subsubsection{Analytical Methods for Cell Culture Performance}

Growth and viability assessment was performed on a Vi-Cell analyzer (Beckman Coulter, Brea, CA) on day 0 and then daily from day 3 to 14 . For product titer measurements the samples were collected daily from day 5 through 14 and analyzed, using a Biacore $C$ instrument (GE Healthcare, Waukesha, WI). Extracellular glucose, lactate and ammonium levels were measured on day 0 and from day 3 to 14 daily. The collected samples were centrifuged and the $0.2 \mu \mathrm{m}$ filtered supernatant was analyzed by Nova Bioprofile 100+ (Nova Biomedical, Waltham, MA).

\subsubsection{Glycan Analysis}

The collected samples from each 3.5-L bioreactor run from day 3 to day 14 were centrifuged, the supernatant $0.2 \mu \mathrm{m}$ filtered and purified on small-scale Protein A affinity columns (PhytipsVR, PhyNexus, San Jose, CA), and then eluted in $20 \mathrm{mM}$ citric acid, $20 \mathrm{mM} \mathrm{PO}_{4}^{3-}$ buffer. The samples were neutralized in $0.5 \mathrm{M}$ Tris. The neutralized samples from day 3 to 7 were concentrated, using Vivaspin 500 30,000 MWCO pore size (Sartorius Stedim, Göttingen, Germany). Both the concentrated (days 3-7) and the non-concentrated neutralized eluates (days 8-14) were denatured by indole-3-acetic acid in $0.6 \mathrm{M}$ denaturation reagent (GlykoPrep-plus, Europa Bioproducts, Cambridge, UK) and reduced. Following purification, the samples were labelled with 2-amino-benzamide and then dried for 3 days. The dried samples were dissolved in $50 \%$ acetonitrile and subsequently injected into the $100 \mathrm{~mm}$ UPLC column in length supplied by Waters Corporation, Milford, MA and eluted, using a gradient.

\subsubsection{Non-Targeted Metabolite Profiling}

Cells were quenched at each time point in $\mathrm{NaCl} 0.9 \%(\mathrm{w} / \mathrm{v})$ at $0.5^{\circ} \mathrm{C}$. The supernatant (for exometabolome analysis) was removed and flash-frozen in liquid nitrogen and stored at $-80^{\circ} \mathrm{C}$. The cell pellet (for endometabolome analysis) was washed in $\mathrm{NaCl} 0.9 \%$, after centrifugation the supernatant discarded and the cell pellet flash-frozen in liquid nitrogen and stored at $-80^{\circ} \mathrm{C}$. Pellets were resuspended in $500 \mu \mathrm{L} 80 \%$ methanol $\left(-20^{\circ} \mathrm{C}\right)$ including internal stan-

Table 8.2 - Feed supplementation of the four 3.5-L bioreactor fed-batch processes.

\begin{tabular}{cccccc}
\hline Process & Feed & Asn $(-)$ & TE $(-)$ & Mn $(\mu \mathrm{M})$ & Gal $(\mathrm{mM})$ \\
\hline A & CD-feed & $1 \times$ & $1 \times$ & 0 & 46 \\
B & CD-feed & $2.7 \times$ & $1.6 \times$ & 5 & 0 \\
C & CD-feed & $1 \times{ }^{\text {a }}$ & $2.9 \times$ & 12 & 0 \\
D & CD-feed & $2.7 \times$ & $3.5 \times$ & 5 & 0 \\
\hline
\end{tabular}

${ }^{\text {a }}$ An additional Asn feed was added on days 3 and 5. 
dards. For metabolite extraction, the cells were disrupted by three freeze-thaw cycles, vortexed and centrifuged at $4{ }^{\circ} \mathrm{C}$ at $13,500 \mathrm{rpm}$ for 5 minutes. An aliquot of $200 \mu \mathrm{L}$ was taken and stored at $-80{ }^{\circ} \mathrm{C}$ for further GC- and LC-MS analysis. Medium samples were prepared by thawing the samples on ice and adding $720 \mu \mathrm{L} 80 \%$ methanol $\left(-20^{\circ} \mathrm{C}\right)$ including internal standards. Samples were vortexed and centrifuged at $4{ }^{\circ} \mathrm{C}$ at 13,500 rpm for 5 minutes. An aliquot of $200 \mu \mathrm{L}$ was taken and stored at $-80{ }^{\circ} \mathrm{C}$ until GC- and LC-MS analysis. Derivatization and analyses of metabolites by a GC-MS 7890A mass spectrometer (Agilent, Santa Clara, CA) were carried out as described $^{297}$. Metabolites were identified in comparison to Metabolomic Discoveries' database entries of authentic standards. The LC separation was performed using hydrophilic interaction chromatography with a ZIC-HILIC $3.5 \mu \mathrm{m}, 200$ A column (Merck Sequant, Darmstadt, Germany), operated by an Agilent 1290 UPLC system (Agilent, Santa Clara, CA). The LC mobile phase was (A) 95\% acetonitrile; $5 \% 10 \mathrm{mM}$ ammonium acetate and (B) $95 \% 10 \mathrm{mM}$ ammonium acetate; $5 \%$ acetonitrile with a gradient from $95 \%$ A to $72 \%$ A at 7 minutes, to $5 \%$ at 8 minutes, followed by a 3-minute wash with $5 \%$ A. The flow rate was $400 \mu \mathrm{L} / \mathrm{min}$, injection volume $1 \mu \mathrm{L}$. The mass spectrometry was performed, using a 6540 QTOF/MS Detector and a AJS ESI source (Agilent Technologies, Santa Clara, CA). The measured metabolite intensities were normalized to internal standards.

\subsubsection{Multivariate Analysis}

SIMCA-P+ (MKS Data Analytics Solutions, Umeå, Sweden) was used for principal component (PCA) analysis of intracellular and extracellular metabolite data and for partial-least-square (PLS) modelling of glycosylation data based on extracellular metabolite profiles. Following univariate analysis of metabolomic data and glycosylation patterns, PCA score and loading plots of intracellular metabolomics data were generated. The purpose of PCA-a multivariate projection method-is the extraction and the representation of the systematic variation in a data set $X$, calling on orthogonal transformation of the data to a new coordinate system that best approximates the data according to equation (8.1) ${ }^{298}$. The $X$ matrix was built with either intra- or the extracellular levels of the four processes, considering the 21 time points throughout each fed-batch process (84 observations in total) and the detected metabolites (both identified and non-identified: 656 variables for intracellular data, 407 variables for extracellular data).

$$
X=1 \cdot \overline{x^{\prime}}+T \cdot P^{\prime}+E
$$

$1 \cdot \bar{x}$ encompasses the variable averages obtained subsequent unit-variance scaling (dividing each value by the column standard deviation) and mean-centering (subtracting the mean of each column), the product $T \cdot P^{\prime}$ comprizes the score matrix $T$, including the scores of the $n$ principal components $\left(t_{1}, t_{2}, \ldots, t_{n}\right)$ and the transposed loading matrix $P^{\prime}$ composed of the loadings of the $n$ principal components $\left(p_{1}, p_{2}, \ldots, p_{n}\right)$, while $E$ represents the noise ${ }^{298}$. The number of principal components (PC) included in the model was defined by optimizing at the same time the goodness of fit $R^{2} X$ and the goodness of prediction $Q^{2} X$. The number of PC is 
given by the maximum of $Q^{2} X$ where the best trade-off between the parameters results. The use of PCA enabled us to identify the metabolites that have a strong influence on the model.

Rather than a maximum least squares projection of $X$ in the case of a PCA, a regression extension of PCA, namely partial least squares projections to latent structures (PLS), or in other terms, a covariance model was built to describe the relationship between $X$ and $Y$ as shown in equation (8.2) ${ }^{298}$. The PLS model was calibrated with the identified extracellular metabolite data of process A, B and D ( $X$ matrix) amounting to 117 variables $(m)$ at the timepoints glycosylation was measured, which corresponds to 36 observations $(k)$. The $Y$ matrix comprized the information related to the temporal glycosylation patterns, including 36 observations $(k)$ and 20 variables $(n)$.

$$
X=\left[\begin{array}{ccccc}
x_{11} & x_{12} & x_{13} & \ldots & x_{1 m} \\
x_{21} & x_{22} & x_{23} & \ldots & x_{2 m} \\
\vdots & \vdots & \vdots & \ddots & \vdots \\
x_{k 1} & x_{k 2} & x_{k 3} & \ldots & x_{k m}
\end{array}\right] \quad \stackrel{\text { PLS }}{\longrightarrow} \quad Y=\left[\begin{array}{cccc}
y_{11} & y_{12} & \ldots & y_{1 n} \\
y_{21} & y_{22} & \ldots & y_{2 n} \\
\vdots & \vdots & \ddots & \vdots \\
y_{k 1} & y_{k 2} & \ldots & y_{k n}
\end{array}\right]
$$

The PLS model is generated by fitting two PCA-like models simultaneously with the aim to model $X$ and $Y$ in addition to predict $Y$ based on the $X$ matrix $^{298}$.

$$
\begin{aligned}
& X=1 \cdot \bar{x}^{\prime}+T \cdot P^{\prime}+E \\
& Y=1 \cdot \bar{y}^{\prime}+U \cdot C^{\prime}+F
\end{aligned}
$$

Likewise, $1 \cdot \bar{x}^{\prime}$ and $1 \cdot \overline{y^{\prime}}$ encompass the variable averages obtained subsequent unit-variance scaling and mean-centering, the score matrices $T$ and $U$ include the observation characteristics, while the variable data is located in both the $X$-loading matrix $P^{\prime}$ and the weight matrix $C^{\prime}$, $E$ and $F$ are called residual matrices, and thereby it is suggested to translate the PLS solution of the latent variable framework as regression model as follows ${ }^{298}$.

$$
\begin{aligned}
Y & =1 \cdot \bar{y}^{\prime}+X \cdot B_{P L S}+F \\
B_{P L S} & =W\left(P^{\prime} W\right)^{-1}=W^{*} C
\end{aligned}
$$

$B_{P L S}$ represents the PLS regression coefficients that are composed of $X$-weight matrix $W^{*}$ and the $Y$-weight matrix $C^{298}$. We defined the number of latent variables-PLS components-to be included in the model by simultaneously optimizing the balance between the goodness of fit $R^{2} Y$ and the goodness of prediction $Q^{2} Y$. PLS regression coefficients, loadings and the variable importance in the projection describe the correlation between the $X$ and $Y$ variables $^{276}$. PLS scatter and score plots were generated to analyze visually the trajectories of the runs included in the model as well as to assess the weight of the specific glycans on the model $(Y)$. As a result of the great number of dimensions of the model, the variable-importance plot (VIP) was used to determine how strongly each of the $117 X$ variables explained both the $X$ and $Y$ space in 
the latent variable model ${ }^{276}$. To further assess the modelling approach and thereby to test all possible permutations, three additional models were built, using the same methodology. One model with processes $\mathrm{A}, \mathrm{B}$, and to predict $\mathrm{D}$, one with $\mathrm{ACD}$ to predict $\mathrm{B}$ and one with $\mathrm{BCD}$ to predict A. Finally, the performance of all four models was assessed, using a two-step validation process. First, a 7-fold cross-validation of each $Y$ variable was conducted. Then, an external data set was included, namely the extracellular metabolite profiles of process $C$ (prediction set) to predict its glycosylation pattern, using the PLS observation model built with the three other runs (calibration set). This step was repeated for the other three models.

\subsection{Results}

\subsubsection{Non-targeted Profiling of Intra- and Extracellular Metabolites}

The four processes A, B, C and D in 3.5-L bioreactors exhibited distinct performance and metabolite responses. Figure 8.1 shows cell growth profiles, viability, titer and three extracellular metabolites. Up to culture day 6, cell growth was comparable in all four process formats (figure 8.1A). Process $\mathrm{C}$ reached the highest viable cell density on day 9 of $24.4 \times 10^{6}$ cells $/ \mathrm{mL}$. At harvest on day 14 , the cell density decreased to $20.3 \times 10^{6} \mathrm{VC} / \mathrm{mL}$. Processes B and D reached slightly lower peak cell densities of respectively 22.4 and $23.3 \times 10^{6} \mathrm{VC} / \mathrm{mL}$. Beyond, both displayed considerably pronounced density decreases, reaching 13.0 and $15.9 \times 10^{6} \mathrm{VC} / \mathrm{mL}$, respectively. Process A stands out from the four runs. The peak was lower $\left(18.8 \times 10^{6} \mathrm{VC} / \mathrm{mL}\right)$ and reached two days earlier, namely on day 7 . At the end of the culture its cell density was in the same range than process $\mathrm{B}$. According to the viability profile in figure $8.1 \mathrm{~B}$, two groups can be identified. Process $\mathrm{A}$ and $\mathrm{C}$ maintained high viabilities throughout the entire culture ( $\geq 90 \%$ ). Starting on day 10 , both B and D distinguish themselves by a much faster decline, going down to 70 and $81 \%$, respectively. Interestingly, the productivity of process A was high. Despite the lower cell growth, the harvest titer amounted to $2.7 \mathrm{~g} / \mathrm{L}$, which was comparable to process $\mathrm{C}(2.8 \mathrm{~g} / \mathrm{L})$ as shown in figure 8.1C. Process D ended up third with a titer of $2.3 \mathrm{~g} / \mathrm{L}$, while process $\mathrm{B}$ yielded $1.9 \mathrm{~g} / \mathrm{L}$. One can also notice that the routine extracellular metabolite analyses highlight different behaviors among the four cell culture processes (figures 8.1D to F). While the glucose profile was comparable for most of the runs, process A was characterized by substantially higher peaks on days 4,7 and 10 . The processes featured three different lactate profile patterns. In all cultures, extracellular lactate levels peaked on day 3 in the region of $2 \mathrm{~g} / \mathrm{L}$. The cells in process A rapidly switched over to lactate consumption, which effected pronounced decrease, reaching levels below the detection limit on day 7 already and remained there until day 14. Process $C$ also eventually switched over to lactate consumption. Nonetheless, lactate levels drop more slowly and were not detected any more, starting on day 10. A slight increase was observed on day 12. The third group is composed of processes B and D. Like process $\mathrm{C}$, the lactate consumption started on day 3 and resulted in a decrease until day 10 when no lactate was detected. However, the cells seemed to favor lactate generation once again 
from day $11 \mathrm{on}$. Process B ended with $0.6 \mathrm{~g} / \mathrm{L}$ lactate in the supernatant and process D reached $0.5 \mathrm{~g} / \mathrm{L}$. In the latter, the increase was not progressive. On day 13 , no lactate was measured. It is not known whether this was a real response or an analytical artefact. The cultivated cells in process A generated the lowest amount of ammonium. Overall, the level progressively climbed up to $7.9 \mathrm{mM}$ on day 14. Process $\mathrm{C}$ showed a more pronounced ammonium secretion until day 6. At that point, it plateaued in the region of 9.4 to $11.2 \mathrm{mM}$. Processes B and D had similar ammonium concentrations throughout the entire culture, reaching high levels. They peaked at about 18 to $19 \mathrm{mM}$ on day 12 and slightly decreased, ending up in the region of 17 to $18 \mathrm{mM}$ of ammonium in the cell culture fluid.

Using non-target metabolite analysis, the next step looked for intracellular and extracellular metabolites, which featured similar patterns to the above presented lactate profiles. Figure 8.2 shows the asparagine (Asn) level changes throughout the culture. One can notice the peak around day 10 of both the intracellular and extracellular levels. Interestingly, process B and $\mathrm{D}$ featured the most pronounced increase of asparagine like they did with lactate, while the asparagine levels only slightly increased in processes A and C. The concentration of this amino acid was the lowest in process $A$ at all times with respect to the other three process formats. The asparagine level seemed to correlate with the lactate concentration. This finding is further supported by the fact that process $\mathrm{C}$ generated intermediate asparagine levels, as was also the case for lactate. A similar pattern showed homocysteine, a sulfur amino acid intersecting two pathways: remethylation to methionine and transsulfuration to cystathionine ${ }^{299}$. Once more, process A stood out due to low intra- and extracellular levels, while intermediate homocysteine levels were observed in process C (figure 8.3). In the supernatant, the levels of processes B and $\mathrm{D}$ were the highest and their levels were globally comparable in the first part of the culture. The intracellular levels were also higher than in the other two process, but process D generated substantially greater amounts. In all processes, an intra- and extracellular increase was observed on day 10. Like in the case of lactate and asparagine, processes A and C induced the smallest change of the homocysteine concentration at that point. B and D resulted in severalfold higher increases.

Figure 8.4 depicts the alanine intra- and extracellular profiles. The intracellular alanine levels of processes A, B and C were comparable the first 3 days. Slightly higher levels were measured in process D. In the supernatant, all four processes were comparable in the beginning. However, the profiles diverged after the first feed addition on day 3. Both in the cell and in the medium of process A, the alanine levels decreased and remained at significantly lower levels. In the other processes, alanine became more abundant inside and outside the cell. Process D exhibited the fastest increase in the cell and remained at the top until the end. The extracellular level of the three processes was comparable throughout the culture. They peaked on day 6 and then progressively decreased. Globally, the extracellular levels correlated with the intracellular levels. High extracellular alanine abundance accompanied high intracellular concentrations. At a few exceptions, the order of the runs in the intracellular profile corresponded to the extracellular environment until day 9 . Then, process $C$ caused the highest alanine levels in the supernatant, while process D remained at the top inside the cell. 

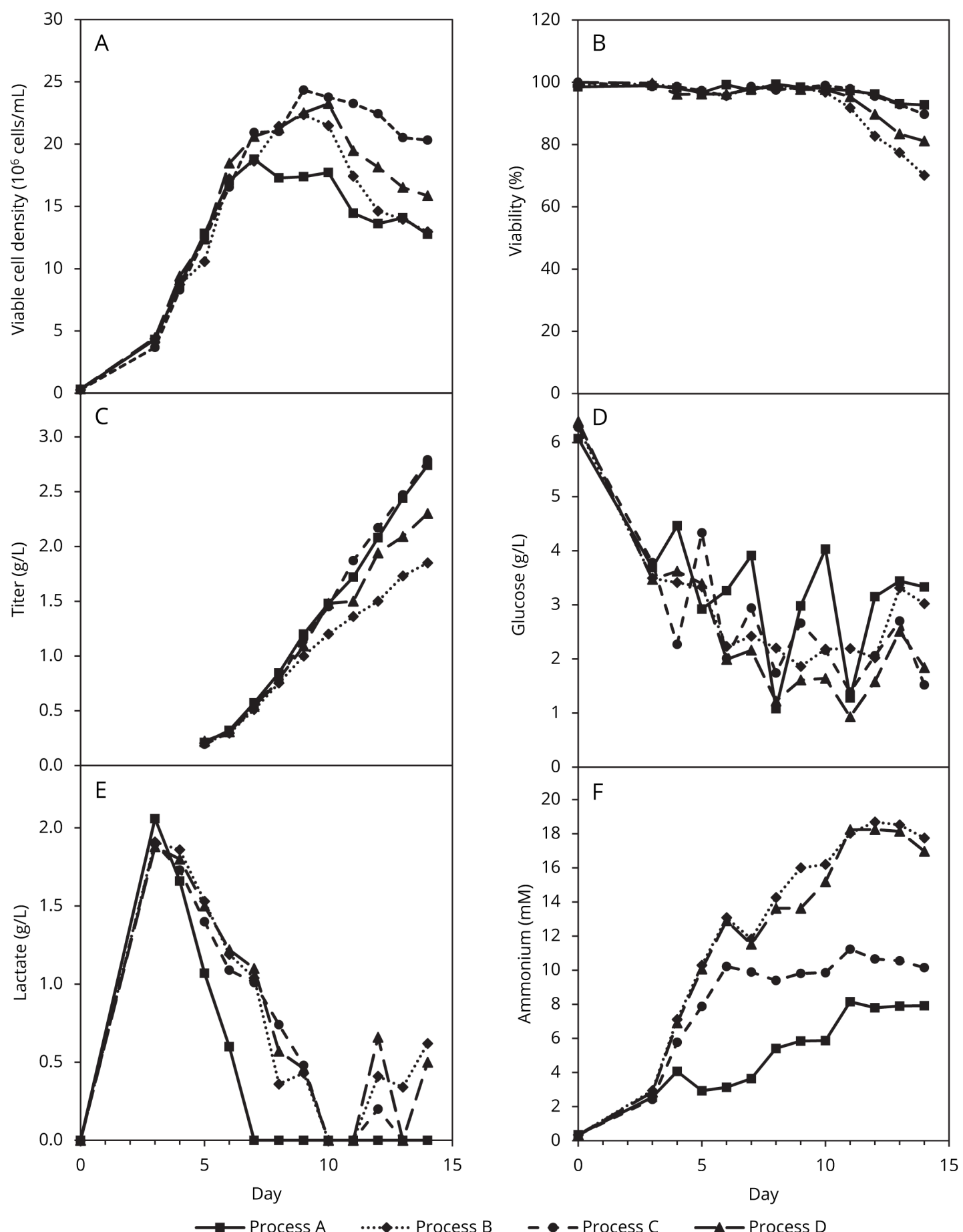

Figure 8.1 - (A) Viable cell densities. (B) Viabilities. (C) Product titer. (D) Extracellular glucose concentration prior to feeding. (E) Extracellular lactate concentration. (F) Extracellular ammonium concentration. All four runs were conducted in 3.5-L bioreactors for 14 days. 


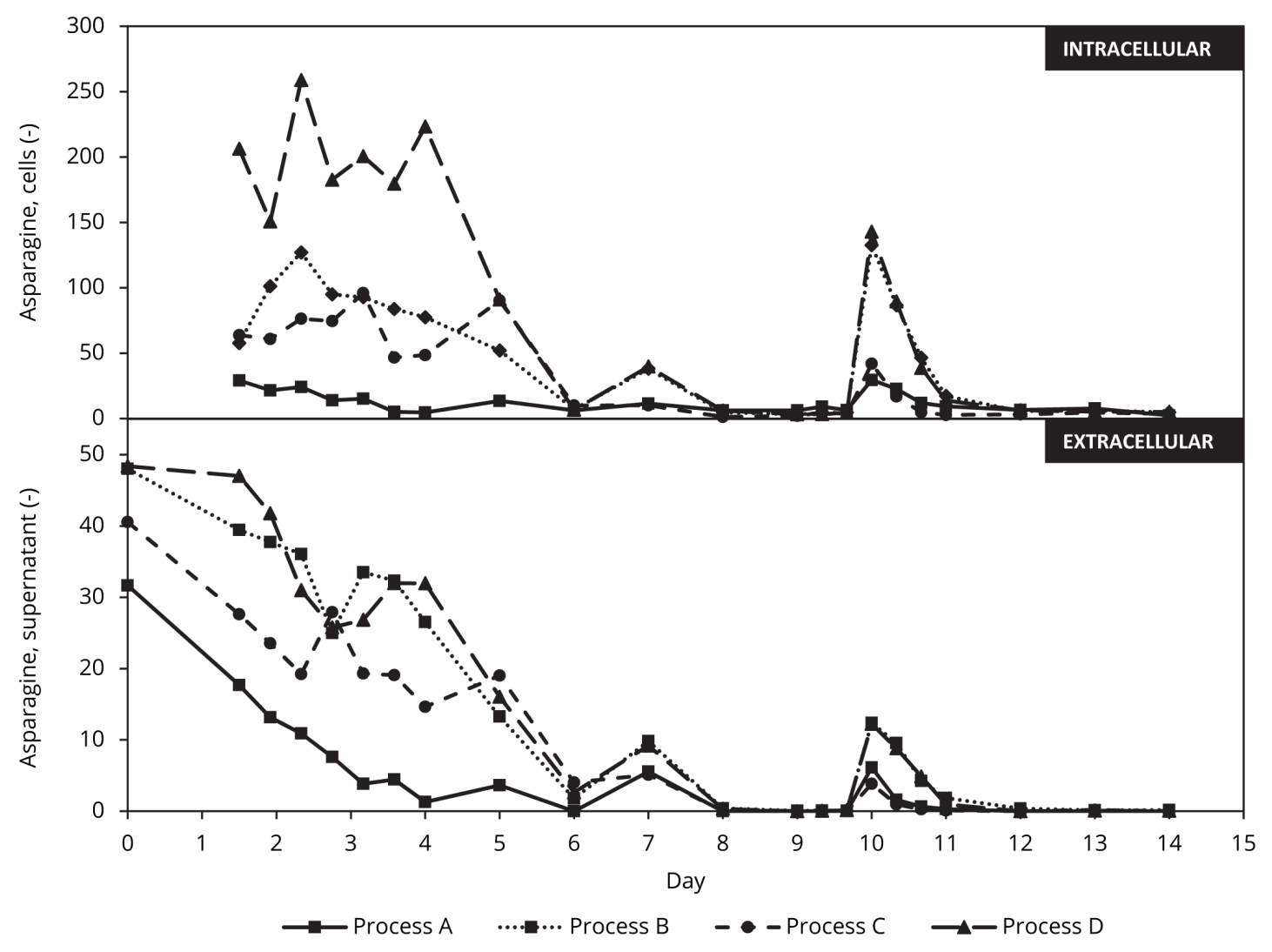

Figure 8.2 - Intracellular (above) and extracellular (below) asparagine profiles. 


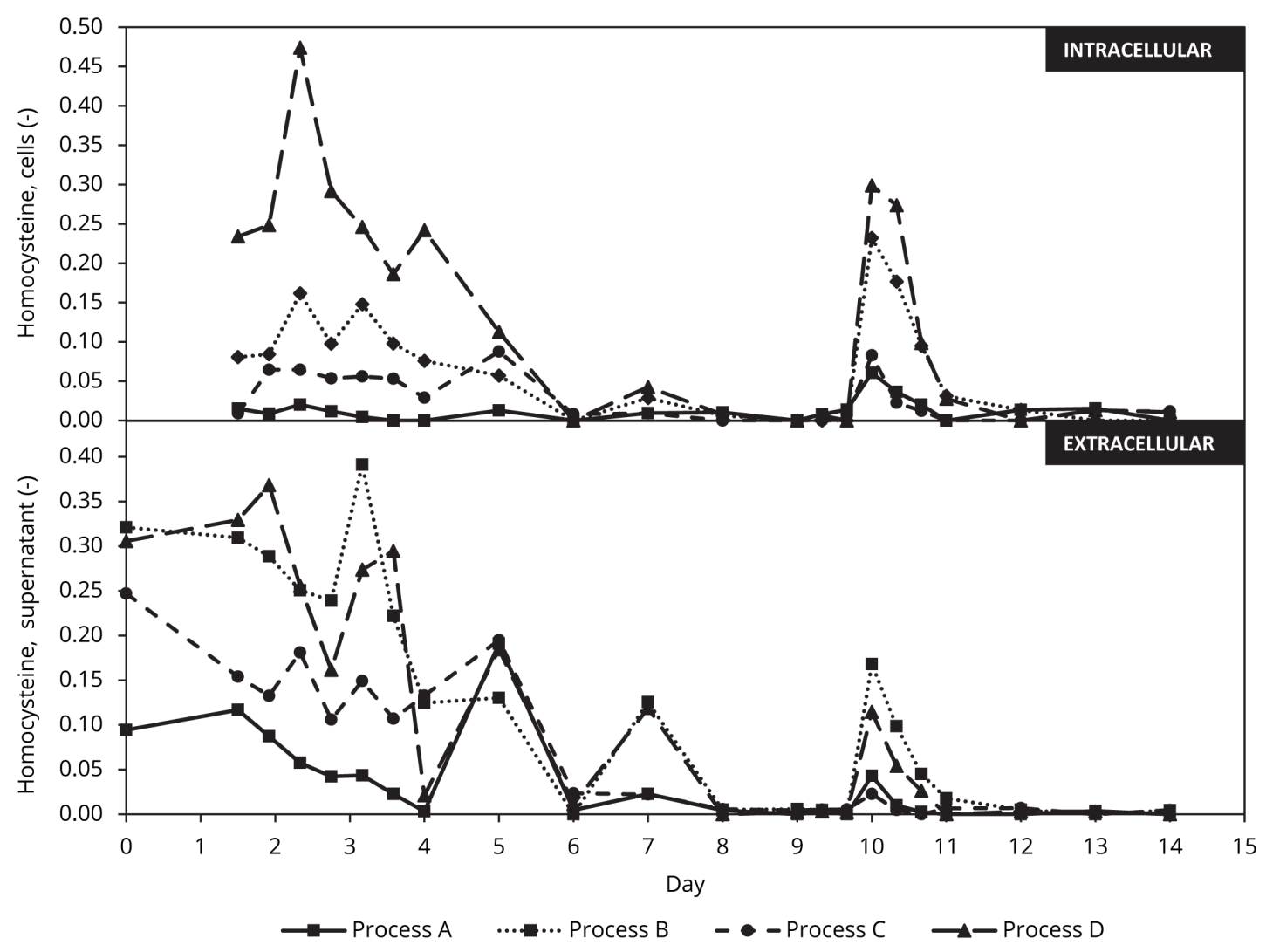

Figure 8.3 - Intracellular (above) and extracellular (below) homocysteine profiles. 


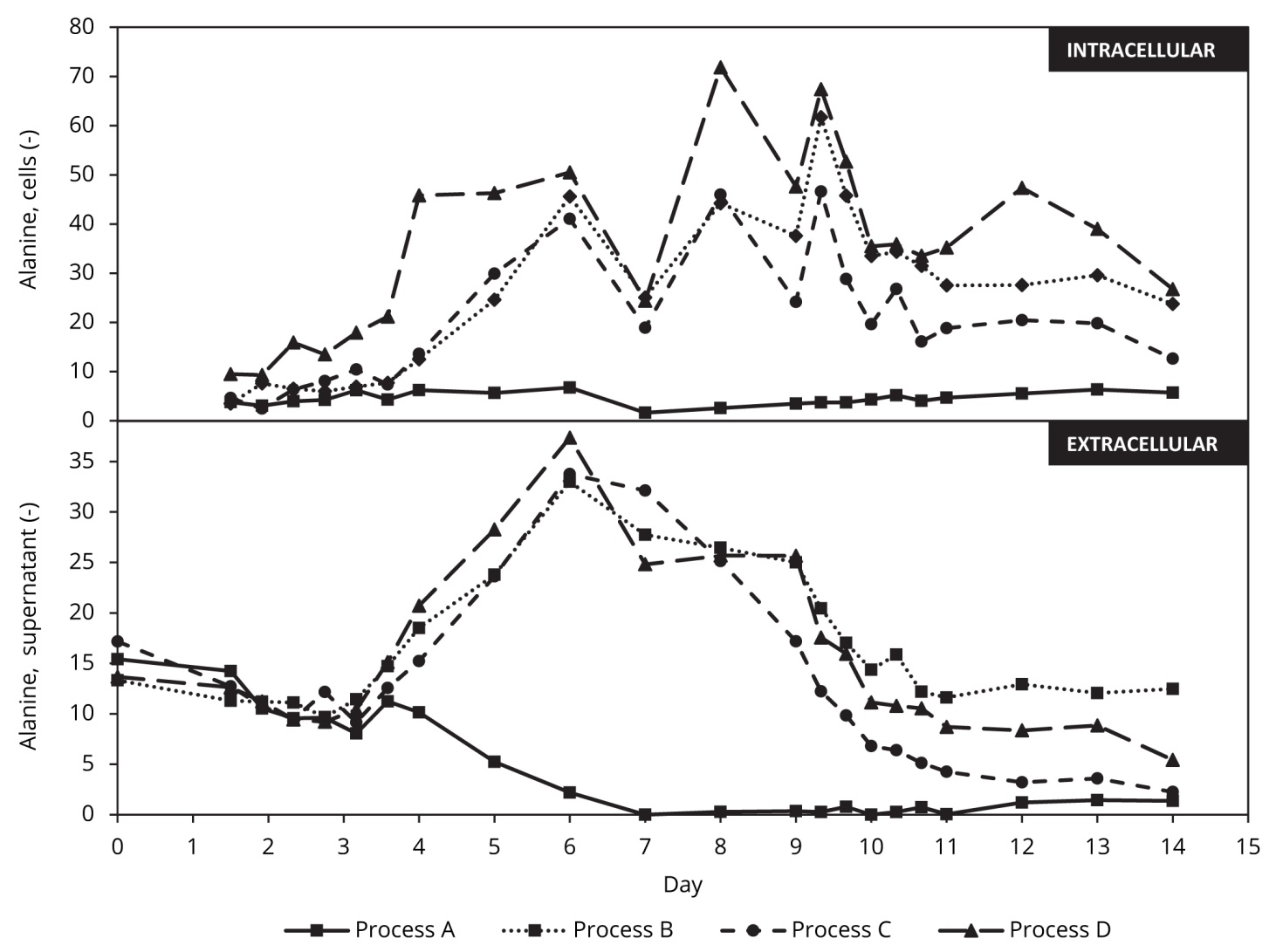

Figure 8.4 - Intracellular (above) and extracellular (below) alanine profiles. 


\subsubsection{Temporal Glycosylation and Nucleotide Sugar Profiles}

At this point, the interest was directed toward the glycosylation fingerprint of each process. The glycan profile was determined once a day, starting on culture day 3 until the end of the run. The fucosylated species FA2-the main glycan peak-and the two galactosylated species, including the mono-galactosylated form FA2G1 (sum of FA2[3]G1 and FA2 [6]G1) as well as the di-galactosylated glycan, FA2G2, in function of time are presented in figure 8.5. The cells readily expressed galactosylated $\mathrm{mAb}$ at the beginning of the culture. On day $3,38.1 \%$ of the $\mathrm{mAb}$ in the supernatant were mono-galactosylated and $4.8 \%$ entirely galactosylated. As the culture progressed, the galactosylation level dwindled slowly. On day 14, galactosylation dropped to $19.6 \%$ of FA2G1 and $1.4 \%$ FA2G2. While exhibiting a distinct progression, processes $\mathrm{B}$ to $\mathrm{D}$ had in common with process A reduced levels of galactosylation at the end of the bioreactor run. Rather than progressively promoting FA2 forms with time, they either started at a similar or slightly lower galactosylation level than process $\mathrm{A}$, and then, in the first part of the culture until day 6 galactosylation levels climbed. Succeeding the peak galactosylation dropped to levels observed in process A. Process B, displayed the lowest galactosylation level of all four processes on day 3, amounting to 29.5\% FA2G1 and 4.5\% FA2G2. Galactosylation peaked at $41.5 \%$ FA2G1 and 6.3\% FA2G2. Both process $\mathrm{C}$ and D expressed comparable amounts of terminal galactose to process A on day 3. They reached higher levels than process B on day 6, amounting to 46.1 and $44.5 \%$ FA2G1, and $6.1 \%$ and $7.0 \%$ FA2G2, respectively. These results show that the abundance of the FA2 glycoform most strongly depended on how readily galactosyltransferase attached galactose to the terminal GlcNAc moiety.

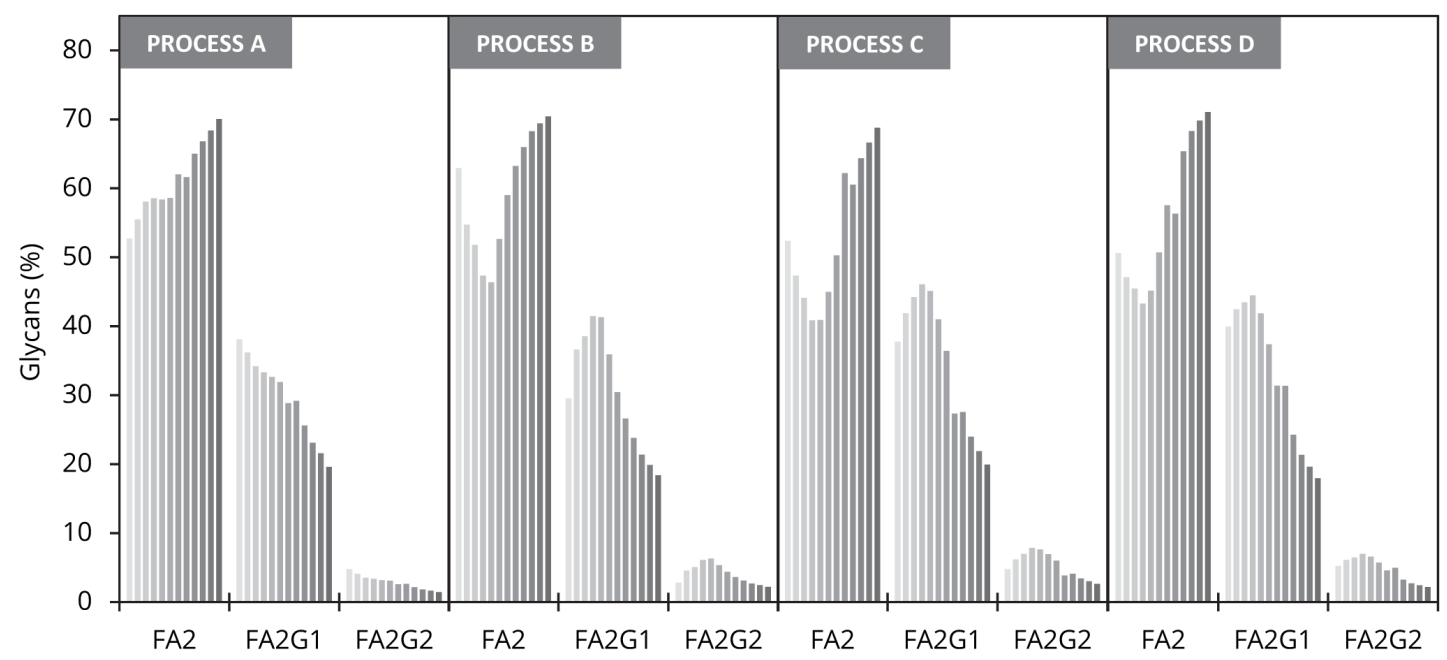

Figure 8.5 - Levels of FA2, FA2G1 (sum of FA2[3]G1 and FA2[6]G1) and FA2G2 in processes A to $\mathrm{D}$ as a relation of the culture time. The glycosylation profile was analysed daily from culture 3 to 14 by $2 \mathrm{AB}-U P L C$.

Although the changes of the mono-galactosylated species were correlated with di-galactosylated species, figure $8.6 \mathrm{~A}$ shows the ratio of the two structures changed during the culture. 
Processes B, C and D expressed the highest ratio of entirely galactosylated glycans around days 6 and 7. At that point, the level of galactosylation peaked. Hence, more abundant galactosylation correlated with higher ratios of FA2G2. The ratios of processes C and D were in the same range on day 3 and at the end of the culture. Process B highly favored mono-galactosylated forms at the beginning and then reached comparable levels to $\mathrm{D}$ towards the end. Process $C$ tended to push the equilibrium towards entirely galactosylated forms. On the contrary, process A exhibited a distinct fingerprint. Like its glycosylation profile, the ratio of FA2G2 versus FA2G1 continually decreased. More abundant glycosylation at the beginning correlated with higher levels of FA2G2, and on day 14, when galactosylation reached the lowest point, the least di-galactosylated antibodies were present in the supernatant. That level was substantially lower than in B, C and D. While all four processes reached comparable galactosylation levels, the ratio of FA2G2 versus FA2G1 of process A was substantially lower, reaching a value of 0.074 . The ratio of the other process formats amounted to $\geq 0.119$.

A

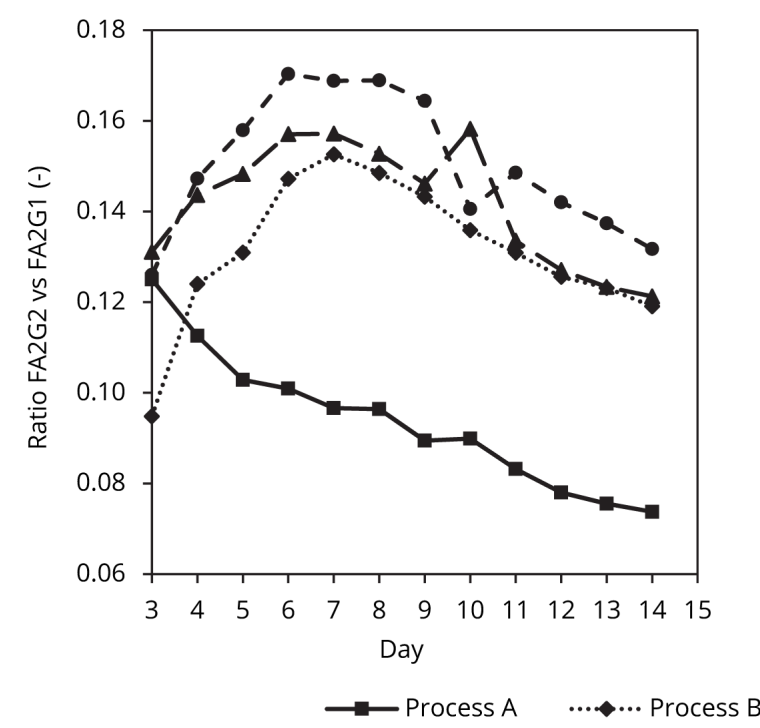

B

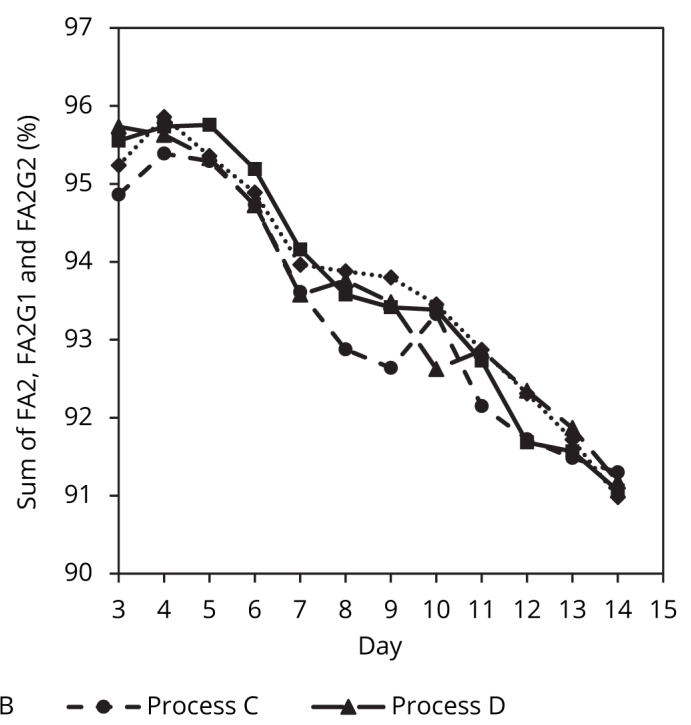

Figure 8.6 - (A) Ratio between di-galactosylated (FA2G2) and mono-galactosylated (FA2G1) forms in function of the culture day. (B) Sum of agalactosylated (FA2), mono-galactosylated (FA2G1) and di-galactosylated forms (FA2G2) in function of the culture day.

Surprisingly, the fucosylated species as a whole were comparable in all four processes throughout the entire fed-batch culture duration. Figure 8.6B highlights that the sum of agalactosylated form FA2, the mono- and di-galactosylated species (FA2G1 and FA2G2) varied between 94.9 and $95.7 \%$ at day 3 and decreased to a narrow range of 91.0 to $91.3 \%$. Theses species remained the main glycans in the entire processes. Nonetheless, the formation of fucosylated species including galactosylation was favored at the beginning, and as the culture progressed, they slightly decreased, while mainly FA1 and mannose 5 more readily appeared (data not shown).

Following the observation of the distinct glycosylation profile trajectories in the respective cell 
culture processes, the levels of glycosylation enzyme substrates and precursors were studied. Figure 8.7 shows the evolution of intracellular UDP-GlcNAc, the substrate of GlcNAc-transferase and the UDP-glucose, a precursor of UDP-galactose. The non-targeted analysis did not pick up the galactosyltransferase substrate, UDP-galactose. Comparable and low levels of UDP-GlcNAc were recorded among the entire array of processes until day 6 (figure 8.7A). Subsequently, the intracellular levels increased until day 12. Processes B, C and D exhibited a stronger increase than A. Nevertheless, the three former were characterized by comparable levels. The nucleotide sugar concentration was considerably lower in process A. Interestingly, the levels in all four processes started to decrease on day 12 or 13. The decrease was the most pronounced in B. On the other hand, the levels of UPD-glucose levels may provide insight on the distinct galactosylation levels of the four processes. UDP-glucose 4-epimerase reversibly converts UDP-glucose to UDP-galactose ${ }^{300}$ and thus may be linked to the abundance of terminal galactose. Until day 4 , the intracellular levels of UDP-glucose were comparable. Then it dropped in processes $\mathrm{B}, \mathrm{C}$ and $\mathrm{D}$ until day 9, while process A remained at higher levels throughout the entire culture. A slight increase was observed in all processes from day 9 to the end of the culture.

A

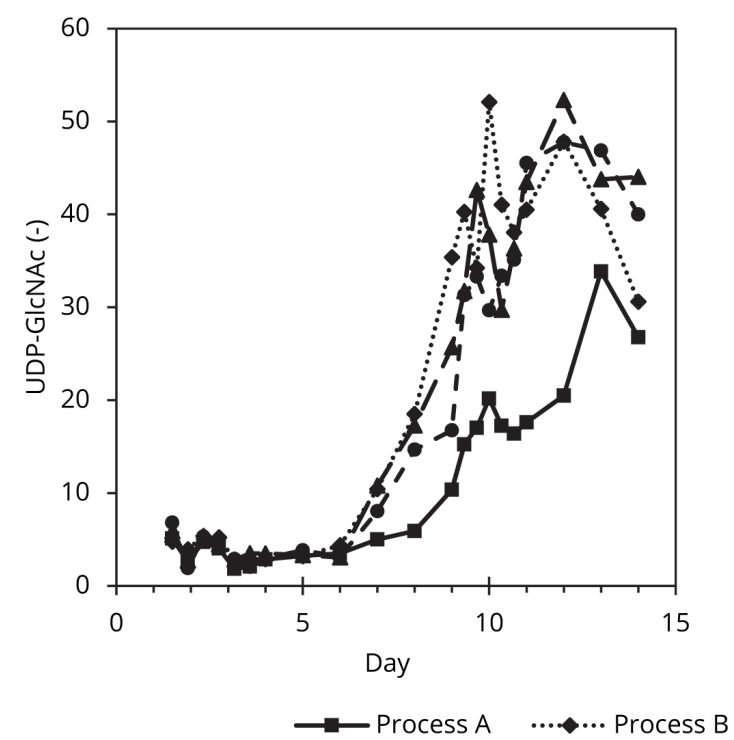

B

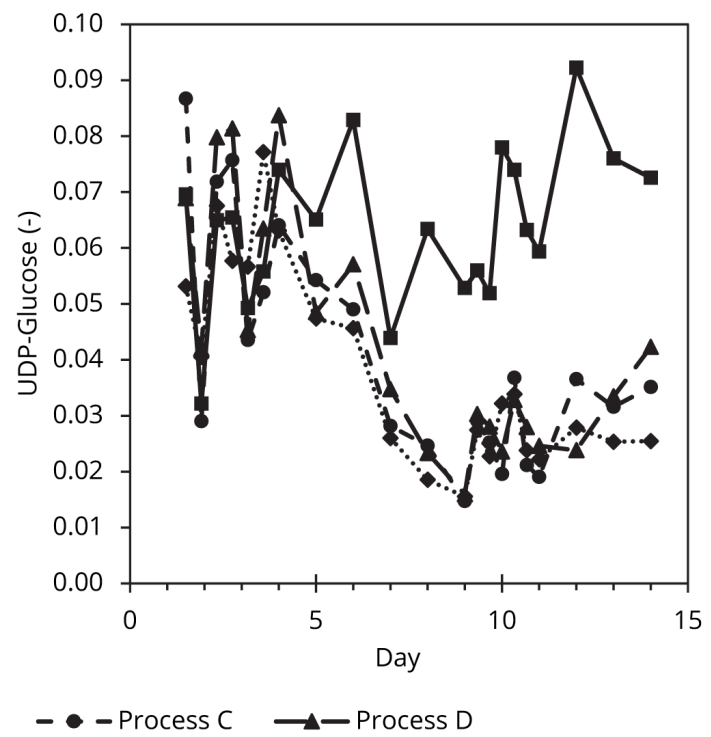

Figure 8.7 - (A) Intracellular UDP-GlcNAc profile throughout the cell culture of processes A to D. (B) Intracellular UDP-glucose profile throughout the cell culture of processes A to D.

The purpose of this chapter is to provide an overview of the metabolic profiles, and in particular, to identify a way to control the glycosylation pattern in routine production. The short summary hereafter describes how the four 3.5-L bioreactor process formats affected the cell metabolism ${ }^{1}$. Process A exhibited low concentrations of alanine, asparagine, homocysteine as well as glutamine and malic acid at the exception of glycine, beta-alanine, inositol and

1. Summary according to analytical service provider's conclusions. 
2-hydroxy-glutarate. Process B affected glutamine levels and urea-cycle linked metabolites arginine, ornithine and agmatine. Furthermore, it produced the highest amount of ammonium. Process $C$ influenced the sugar metabolism, entailing accumulation sugars and sugar alcohols at late time points. Citrate and isocitrate, lysine, arginine, proline, methionine and cadaverine were also more readily accumulated in comparison to the other processes. Finally, the strongest effects were observed in process $\mathrm{D}$ where many pathways were affected, including the TCA-cycle, methionine and homocysteine, beta-alanine and pantothenate, the urea-cycle, homoserine and threonine and also highly linked glutamate and glutamine. The extracellular levels of all processes did not always reflect the endometabolic trends.

\subsubsection{Multivariate Analysis and Modelling}

Thereby, the four fed-batch processes showed different fingerprints of both a selection of intracellular metabolites including nucleotide sugars and glycosylation pattern trajectories. Aiming to identify a way to control glycosylation during production, it was evaluated to what extent multivariate analysis can be used to predict the observed differences and to reduce the complexity. Figure 8.8 presents the PCA score plot of the intracellular metabolites ( $X$ variable). The first principal component (PC1) explains $44 \%$ of variance and the second (PC2) $7 \%$. Early time points were located in the upper left quadrant. Intermediate points were projected in the lower left and right quadrants. Late time points of process A remained in the lower right quadrant, highlighting the distinct metabolite profiles in comparison to the other process formats. The score plot also depicted the intermediate pattern of process $\mathrm{C}$. In general, its trajectory followed processes B and D. But at the end, it approached the central point rather than further progressing upwards and to the right. Process D exhibited a distinct trajectory in the lower left quadrant and ended up most distant to the center on culture day 14 . The first two PC of the score plot including the extracellular metabolites explain $26.6 \%$ and $11.6 \%$, respectively, of variance (data not shown).

Subsequently, a partial-least-square model was built that may be used to control the glycosylation pattern in routine production. Rather than using intracellular metabolite data, it was preferred to calibrate the model exclusively with extracellular metabolite profiles of processes A, B and D and their respective glycosylation profiles. This choice was driven by practical reasons. In routine production, the measurement of intracellular metabolites would be too complex. Moreover, various existing on-line and at-line technologies are suitable to acquire extracellular data. The first step in the PLS model building consisted of the determination of the optimal number of latent variables. The optimal number was located in the region of 3 to 4 , where the best balance between the goodness of fit $\left(R^{2} X\right.$ and $R^{2} Y$ ) and the goodness of prediction $\left(Q_{\text {cum }}^{2}\right)$ was encountered (cf. table 8.3). To avoid any overfitting, the PLS observation model was built with the first three components only. Although the first two components of the PCA- $X$ of extracellular data explained less variance when using intracellular data (41 vs. 51\%), figure 8.9 shows that the goodness of fit of the first two components of the model amounted to $39 \%$ and $10 \%$, which summed up to almost $50 \%$ of the variance. The third latent variable 


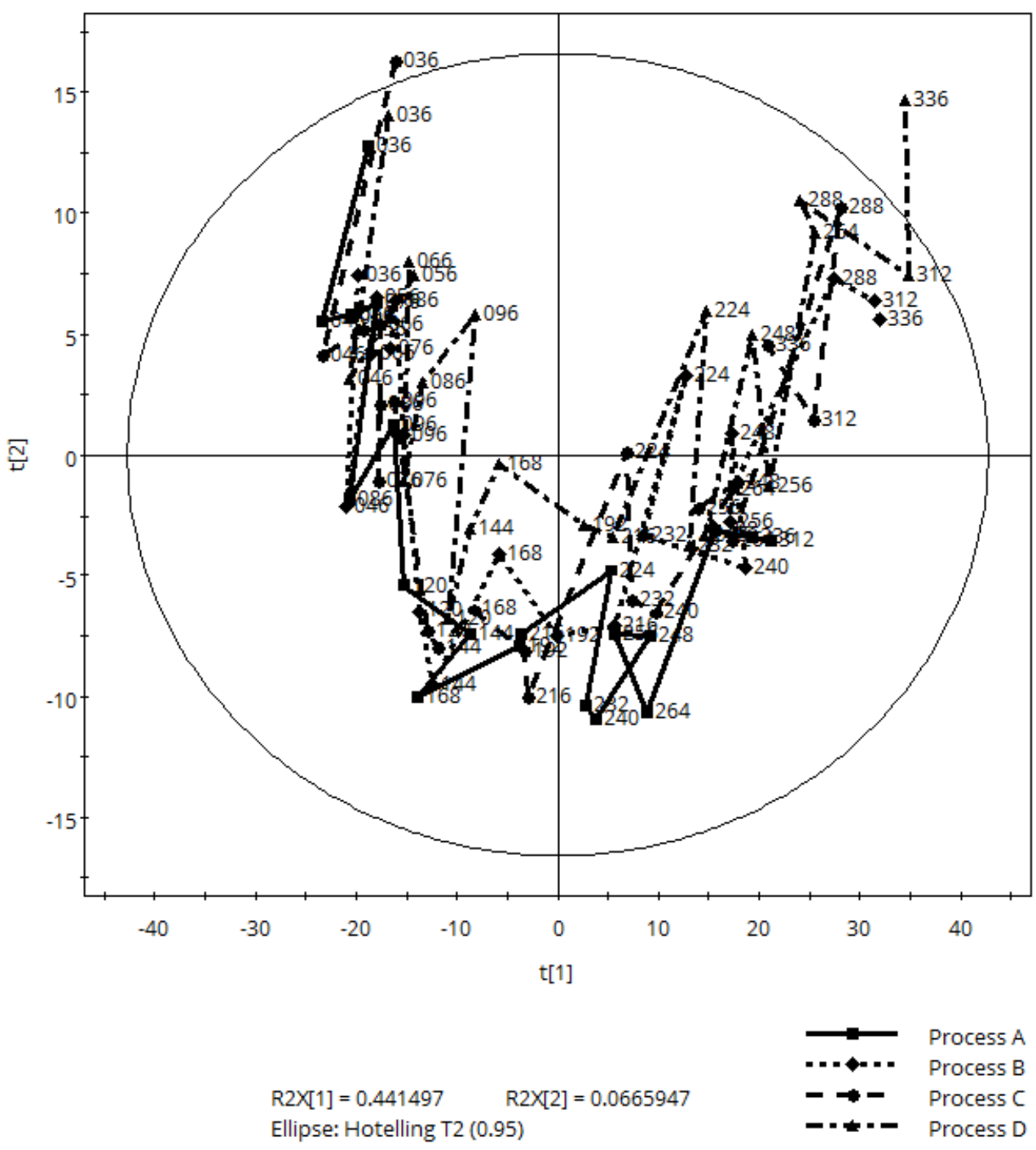

Figure 8.8 - PCA-X score plot of processes A to D. The PC1 explains $44 \%$ of variance, and PC2 $7 \%$. Each time point is labelled with the respective culture hour of the corresponding process format. The ellipse delimits the $0.95 \%$-confidence area. 
adds additional $6 \%$ of variance explained by the model. It is important to notice the absence of strong outliers. In the score plot, the 95\%-confidence ellipse shows the operating area defined by Hotelling's $T^{2298}$. All observations are within that area. The weight plot of the model in figure 8.10 shows that the galactosylated species are located in the upper left quadrant, which means that galactosylation was favored in the beginning of the culture. The early time points are located at the left in the score plot. The observer's eye catches the cluster in the lower right quadrant, including high mannoses (Man5 and 6) and fucosylated glycans (FA1 and FA2). The late time points of the three processes can be found in the lower right quadrant of the score plot. Hence, the model shows that with progressing cell culture, the cells more readily expressed high mannose and fucosylated forms containing antibodies, while galactosylation was reduced towards the end. A2 and Man7 can also be found on the right-hand side of the weight plot. Notice the different trajectories of process A at 144 and 168 hours (days 6 and 7) accounting for the distinct profiles of some extracellular metabolites. According to the variable importance plot in figure 8.11, the metabolites having the greatest influence in the model are glycerol, arabitol, glucoronic acid and homoserine.

Table 8.3 - Goodness of fit $\left(R^{2} X\right.$ and $\left.R^{2} Y\right)$ and goodness of prediction $\left(Q_{\text {cum }}^{2}\right)$ of PLS model in function of the number of latent variables.

\begin{tabular}{cccc}
\hline \# Latent Variables & $R^{2} X$ & $R^{2} Y$ & $Q_{\text {cum }}^{2}$ \\
\hline 1 & 0.387 & 0.444 & 0.413 \\
2 & 0.488 & 0.640 & 0.564 \\
3 & 0.553 & 0.762 & 0.668 \\
4 & 0.638 & 0.792 & 0.691 \\
5 & 0.691 & 0.812 & 0.671 \\
\hline
\end{tabular}

The small number of batches prevented building a batch model. The resulting model quality was poor (data not shown). The observation model was used instead to predict the glycosylation profile of process $\mathrm{C}$ based on the metabolite data. The model can be used to predict the glycan pattern at a specific day based on the extracellular metabolite levels at that moment. However, the model is not capable to predict the final glycan fingerprint at the very end of the fed-batch process. To assess the prediction quality of the four major glycan species, FA2, FA2 [6]G1, FA2[3]G1, and FA2G2, the observed versus predicted value plots in figure 8.12 provided insight at each specific glycan value. The predicted points of FA2[3]G1 lie rather closely to the diagonal line, whereas for the points of the other three species form a curve. Both at the extremes and in the middle the prediction error is larger as the larger distance between the points and the line indicates. For instance, at 55 and $70 \%$ of FA2 the prediction is good.

Two time points of the culture were chosen to assess the difference of the experimental and the predicted glycosylation profiles of process $C$ as shown in figure 8.13. On day 6, the FA2 and the galactosylated glycan peaks of the PLS-model calibration set processes exhibited the largest differences (cf. section 8.3.2). At this time point, the model overestimates the FA2 peak (figure $8.13 \mathrm{~A}$ ). While $40.8 \%$ of the secreted antibodies on day 6 held a FA2 glycan, the 


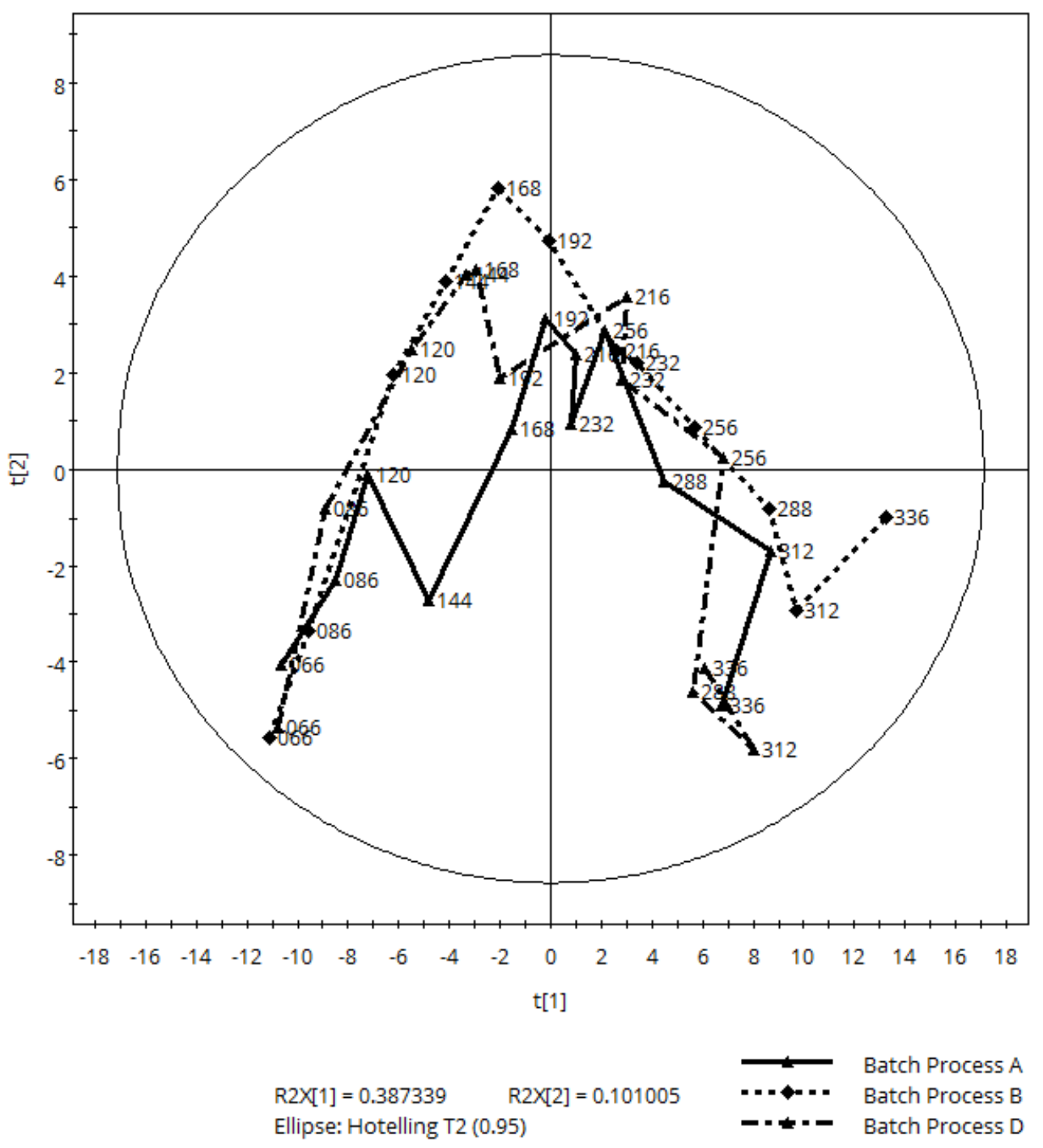

Figure 8.9 - PLS scatter plot of processes A, B and D. The goodness of fit $\left(R^{2} X\right)$ of the first component amounts to $39 \%$ and the second component to $10 \%$. Each time point is labelled with the respective culture hour of the corresponding process format. The ellipse delimits the 0.95\%-confidence area. 


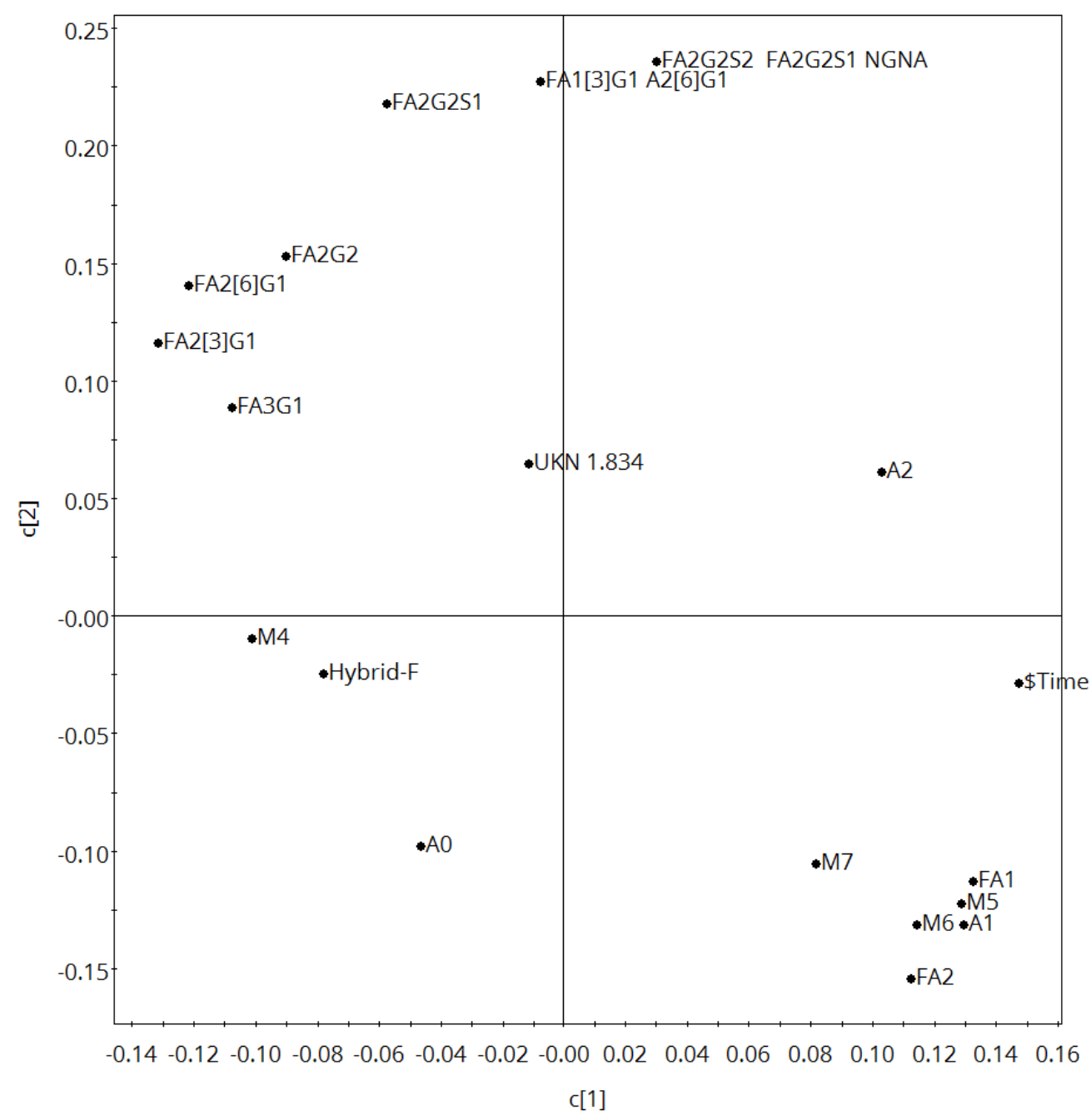

$\mathrm{R} 2 \mathrm{X}[1]=0.387339 \mathrm{R} 2 \mathrm{X}[2]=0.101005$

Figure 8.10 - PLS weight plot of processes A, B and D. The goodness of fit $\left(R^{2} X\right)$ of the first component amounts to $39 \%$ and the second component to $10 \%$. 


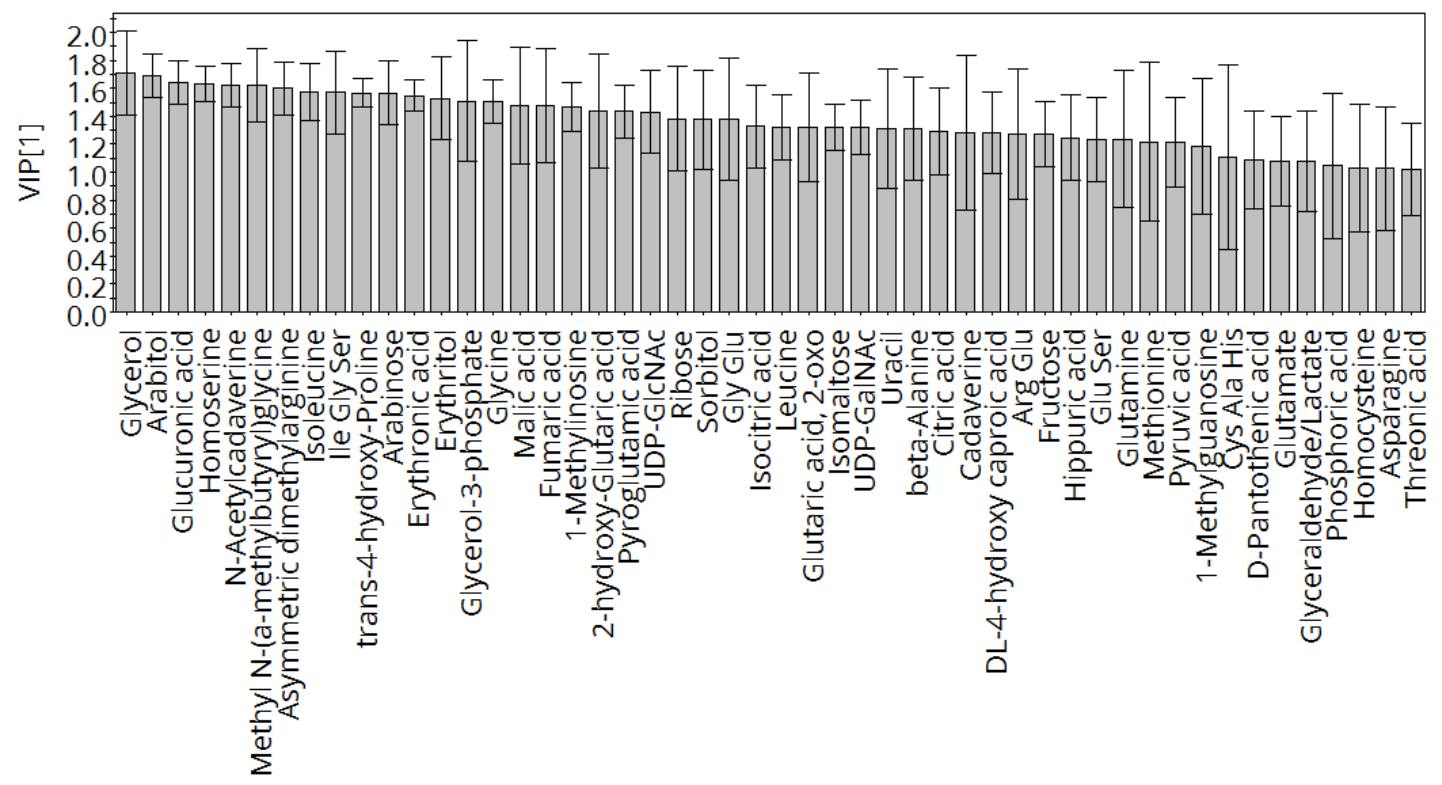

Figure 8.11 - Variable importance plot. The chart includes the variables above 1 . The error bars indicate the $95 \%$ confidence intervals.

model estimation amounted to $51.7 \%$. Galactosylation was underestimated: the experimental values of the monogalactosylated forms FA2 [6]G1 and FA2[3]G1 were 34.7 and $11.3 \%$. The model predicted 28.2 and $9.8 \%$, respectively. In process C, di-galactosylation reached $7.8 \%$, whereas $5.2 \%$ were predicted. The model exhibited a quite well prediction accuracy for A2 (1.3 vs. 1.2\%) and the minor peaks FA2G2S2 FA2G2S1 NGNA, FA3G1 and Man5. At the end of the culture, on day 14, the model quality was substantially improved, as shown in figure 8.13B. The abundance of the main glycan peak - the agalactosylated form FA2-was perfectly well predicted. At harvest, $68.8 \%$ of the mAbs were agalactosylated. The PLS model prognosticated a slightly lower percentage, adding up to $68.0 \%$. Although to a smaller extent, it still overestimated mono-galactosylated species FA2[6]G1 (14.4 vs. 15.9\%) and FA2[3]G1 (5.5 vs. 6.0\%). Nonetheless, the level of di-galactosylated glycans was well estimated. Both the experimental and the predicted values amounted to $2.6 \%$. The model also proved to be reliable for Man5, estimating $1.5 \%$, which is nearby the experimental value of $1.4 \%$. A2 and FA1 were slightly underestimated, resulting in differences $\leq 0.8 \%$ between experimental and predicted values. The comparison of the major glycan species FA2 between the four models that resulted from permutation of the processes included in the calibration set in figure 8.14 depicts an overall good prediction accuracy. On day 6 , the calibration set including processes A, B and D featured the best congruence with the prediction set. Like in the case of univariate analysis, process A distinguished itself once more. When process A was part of the calibration set, FA2 abundance was overestimated, while the model including B, C and D underestimated the FA2 of process A. The prediction was greatly enhanced on the final culture day. Three of the four models predicted the FA2 level within less than $2 \%$ absolute difference. The distinct characteristics of process A was not apparent. The root mean square error of evaluation (RMSEE), of cross 
A

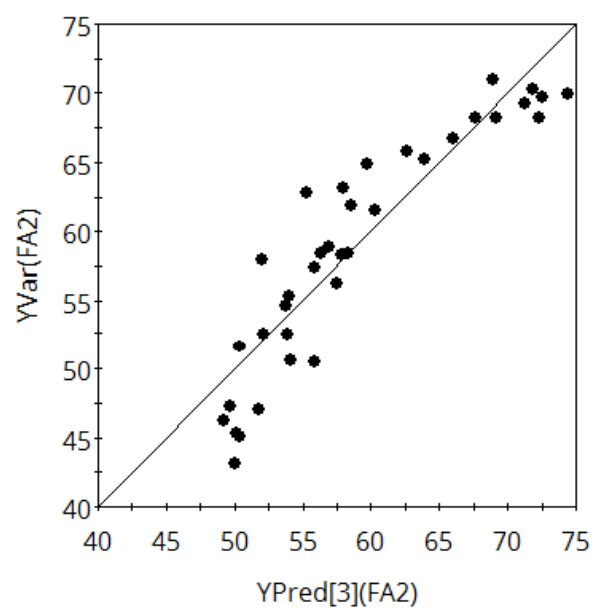

C

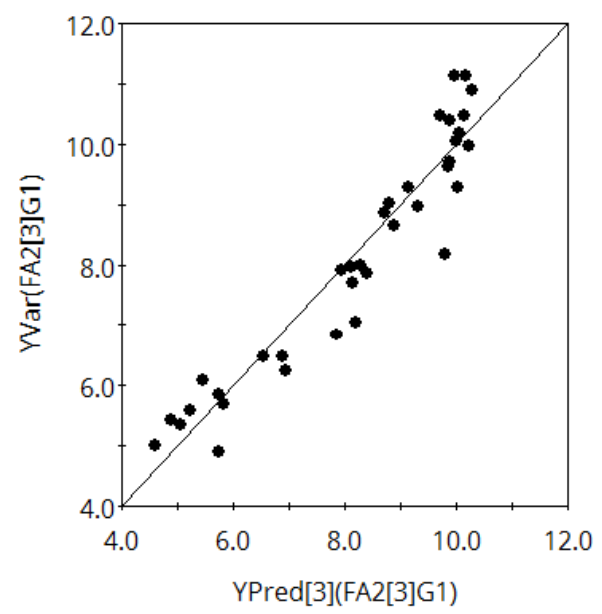

B

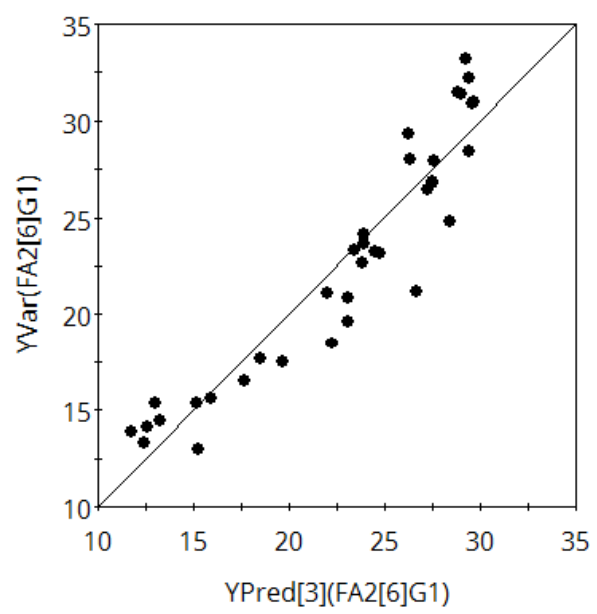

D

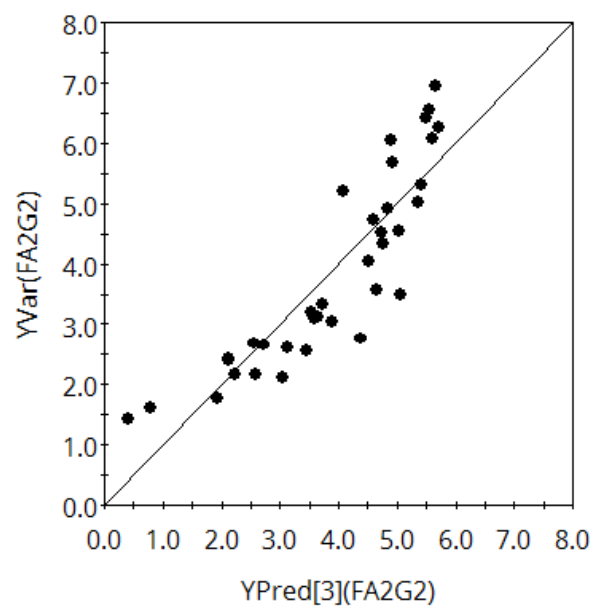

Figure 8.12 - Observed versus predicted values of FA2, FA2[6]G1, FA2[3]G1 and FA2G2. 
validation (RMSECV), and of prediction (RMSEP) of the modelled glycan species were in the same range (table 8.4), and more importantly the RMSEP were lower than the actual range of the predicted values (except for A0, a minor species). In particular, the prediction error was considerably lower than values of the main glycan species FA2 as well as both mono- and di-galactosylated forms.

Table 8.4 - Average PLS observation model errors of each glycan species \pm 2 standard deviations taking into account all four models.

\begin{tabular}{lcccc}
\hline Glycan & RMSEE (\%) & RMSECV (\%) & RMSEP (\%) & Glycan Range (\%) \\
\hline A0 & $0.01 \pm 0.01$ & $0.01 \pm 0.01$ & $0.01 \pm 0.01$ & $0.00-0.09$ \\
A1 & $0.05 \pm 0.02$ & $0.06 \pm 0.02$ & $0.06 \pm 0.03$ & $0.09-0.61$ \\
A2 & $0.19 \pm 0.05$ & $0.21 \pm 0.06$ & $0.29 \pm 0.23$ & $0.84-2.20$ \\
FA1 & $0.17 \pm 0.08$ & $0.20 \pm 0.08$ & $0.25 \pm 0.16$ & $0.33-2.37$ \\
FA1[3]G1 A2[6]G1 & $0.09 \pm 0.02$ & $0.09 \pm 0.02$ & $0.13 \pm 0.08$ & $0.30-0.91$ \\
FA2 & $3.56 \pm 0.21$ & $3.97 \pm 0.29$ & $5.50 \pm 0.79$ & $40.84-71.05$ \\
FA2[6]G1 & $2.18 \pm 0.21$ & $2.49 \pm 0.26$ & $3.34 \pm 0.14$ & $13.04-34.73$ \\
FA2[3]G1 & $0.59 \pm 0.07$ & $0.68 \pm 0.08$ & $0.81 \pm 0.19$ & $4.92-11.32$ \\
FA2G2 & $0.78 \pm 0.11$ & $0.86 \pm 0.14$ & $1.40 \pm 0.60$ & $1.44-7.84$ \\
FA2G2S1 & $0.09 \pm 0.02$ & $0.10 \pm 0.02$ & $0.15 \pm 0.08$ & $0.40-1.13$ \\
FA2G2S2 FA2G2S1 NGNA & $0.06 \pm 0.01$ & $0.06 \pm 0.01$ & $0.07 \pm 0.03$ & $0.14-0.60$ \\
FA3G1 & $0.02 \pm 0.00$ & $0.02 \pm 0.00$ & $0.03 \pm 0.04$ & $0.21-0.38$ \\
Hybrid-F & $0.03 \pm 0.01$ & $0.03 \pm 0.01$ & $0.04 \pm 0.02$ & $0.01-0.22$ \\
Man4 & $0.02 \pm 0.00$ & $0.02 \pm 0.00$ & $0.02 \pm 0.00$ & $0.03-0.13$ \\
Man5 & $0.19 \pm 0.04$ & $0.22 \pm 0.05$ & $0.21 \pm 0.08$ & $0.27-2.27$ \\
Man6 & $0.04 \pm 0.01$ & $0.05 \pm 0.01$ & $0.06 \pm 0.03$ & $0.05-0.43$ \\
Man7 & $0.04 \pm 0.01$ & $0.04 \pm 0.01$ & $0.06 \pm 0.02$ & $0.20-0.47$ \\
UKN 1.834 & $0.09 \pm 0.07$ & $0.10 \pm 0.09$ & $0.21 \pm 0.28$ & $0.34-1.09$ \\
\hline
\end{tabular}

\subsection{Discussion}

The presented results show that diverse medium compositions substantially altered the metabolism of the cultured cells. The four process formats not only displayed differing cell culture performances including cell growth and productivity, but resulted in a variation of extracellular lactate and ammonium profile shapes. The process format influenced the degree of lactate consumption versus lactate production. The asparagine concentration in the medium was directly correlated with the production of ammonium. The medium of process A contained the lowest amount of Asn of the four 3.5-L bioreactor processes, and as a result, low Asn levels were present in the supernatant. Increased ammonium levels in processes B, C and $\mathrm{D}$ were correlated with supplementary Asn additions either combined with the CD-feed or as a separate feed solution. Processes B and D were characterized by high levels of ammonium $\left(\geq 18 \mathrm{mM}\right.$ ) that were well beyond the reported galactosylation inhibiting levels ${ }^{126,237}$. The 
A

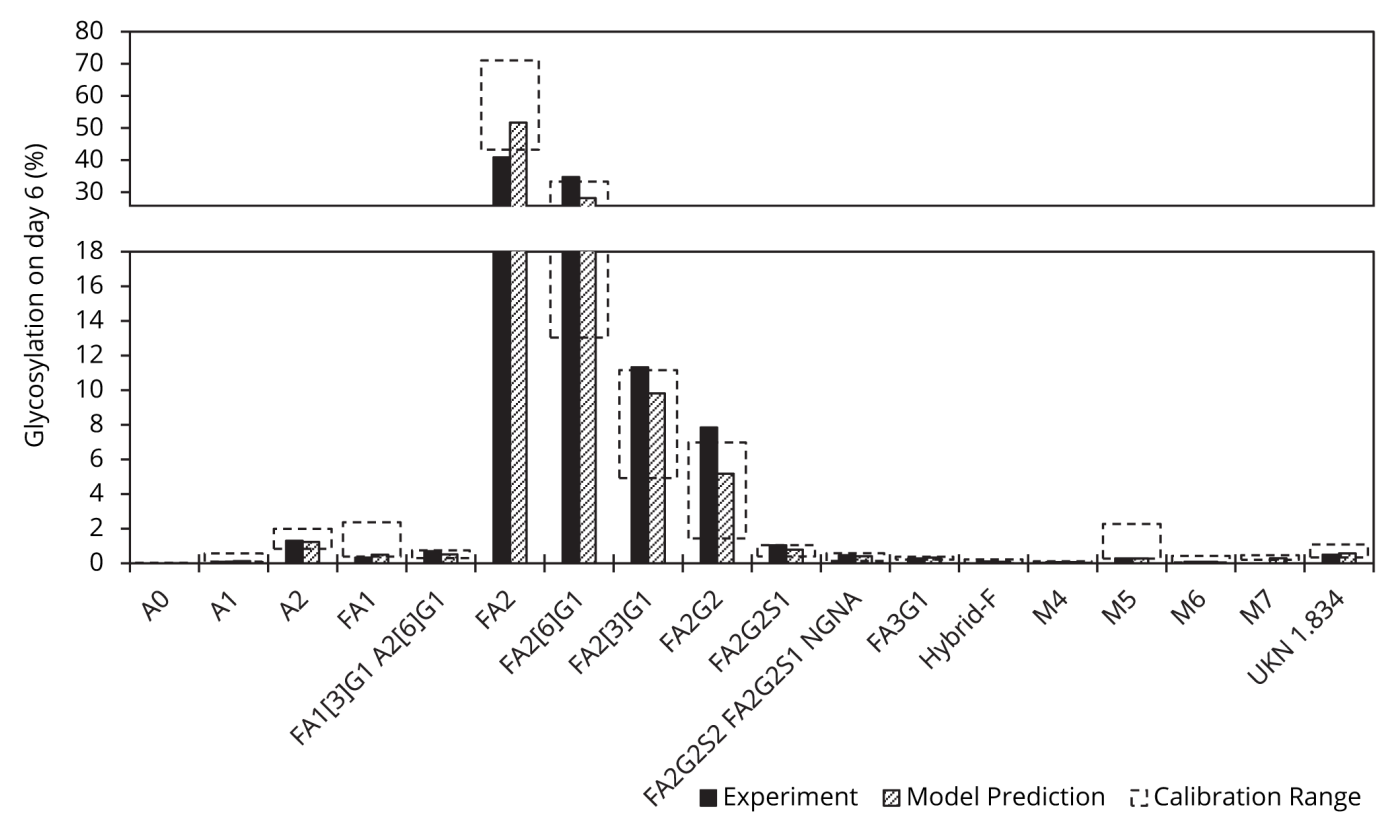

B

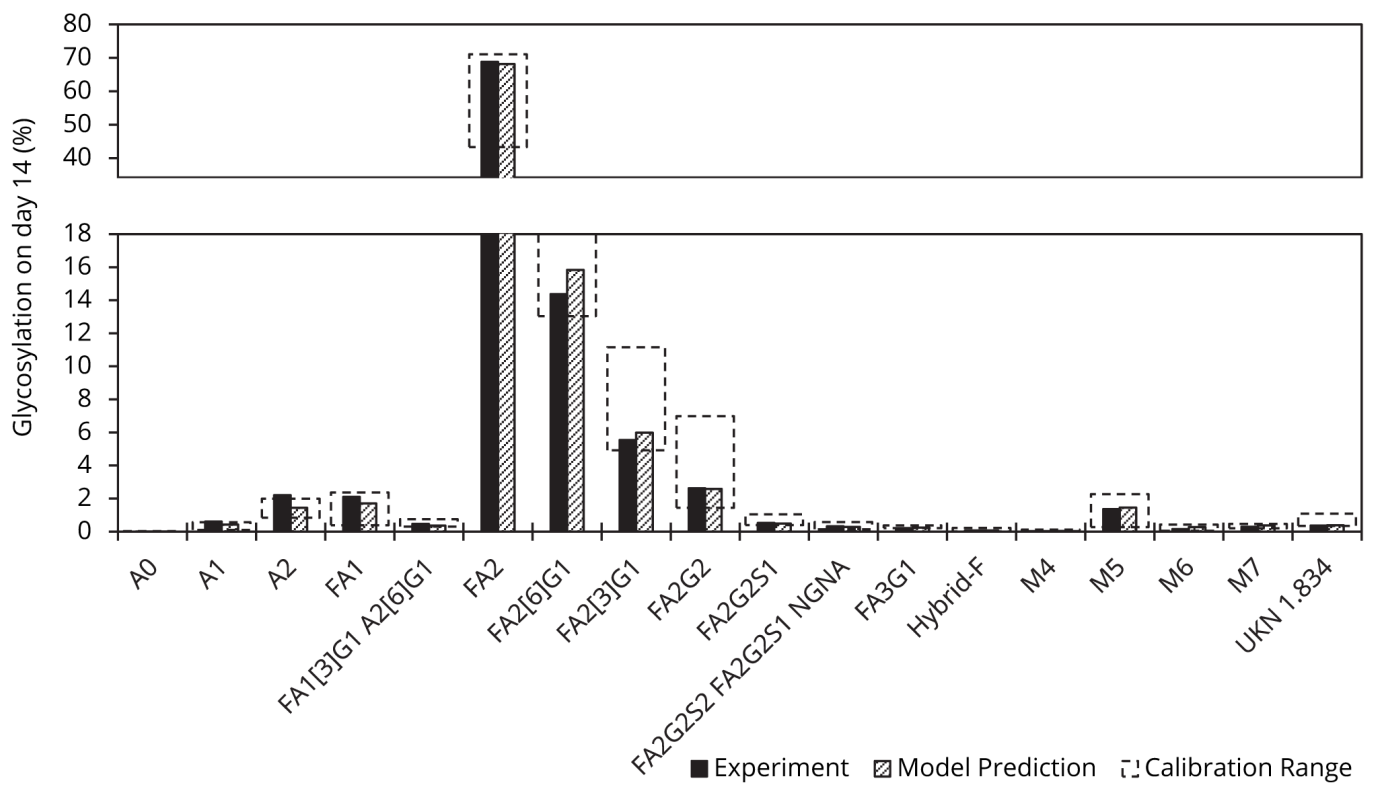

Figure 8.13 - Experimental and predicted glycans of process C by PLS model on days 6 (A) and 14 (B). The model was built with extracellular metabolite data of process A, B and D. The dashed box marks the calibration set range of the corresponding glycan. 
A

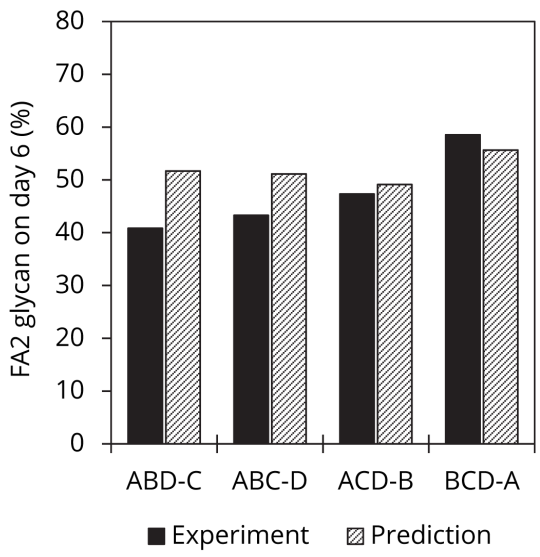

B

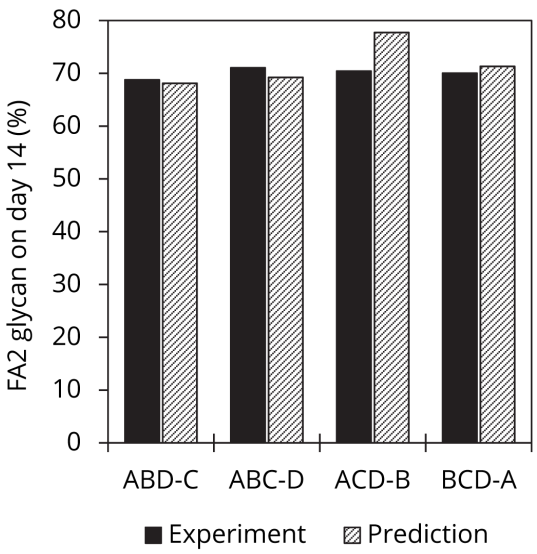

Figure 8.14 - Comparison of experimental FA2 values versus predicted values of the four different models indicated in the chart legend. The first three letters indicate the processes included in the calibration set of the model, while the last corresponds to the predicted process.

lower cell densities as well as reduced productivity suggest that the high ammonium levels entailed toxic effects for the cells. The literature reported that ammonium levels $\geq 10 \mathrm{mM}$ effected the cell culture performance, and in particular at $20 \mathrm{mM}$ dramatic effects were observed $^{301}$. Therefore, higher galactosyltransferase activity and gene expression in processes A and $\mathrm{C}$ is assumed. It is possible that manganese supplementation compensated the reduced activity in processes $\mathrm{B}$ and $\mathrm{D}$, thus allowing to reach comparable levels of galactosylated glycans at the end of the culture. Nonetheless, it seems that even at important ammonium levels, galactose was still attached to the terminal GlcNAc of the glycan backbone. On day 6 , when the galactosylation was the most abundant, the product titers were in the range of 0.30 to $0.32 \mathrm{~g} / \mathrm{L}$. Until harvesting, the secreted mAb concentrations increased by six to nine times. At the same time, galactosylation decreased only 1.7- to 2.6-fold. Thus, an important number of galactosylated mAbs was produced between days 6 and 14, while in particular in processes $\mathrm{B}$ and $\mathrm{D}$ ammonium levels were aloft, climbing up to levels substantially beyond the reported threshold of $10 \mathrm{mM}$. The glycosylation profiles of the four 3.5-L fed-batch experiments indicate that process optimization including media and feed design allowed to reach the same glycosylation endpoint, starting the culture at different levels. Although the sum of agalactosylated, mono-galactosylated and di-galactosylated forms was comparable among the tested processes, it decreased as the culture progressed, showing that the generation of other species became more frequent. Moreover, the degree of galactosylation-the ratio of FA2G2 versus FA2G1 - was higher with increasing percentage of galactosylated antibodies and strongly dependent on the process format. Despite the galactose containing feed of process A, the absence of manganese seemed to impede the attachment of galactose to the terminal GlcNAc. The ratio of di-galactosylated and mono-galactosylated forms of process A amounted to about 0.08 . In B, C and it was greater than 0.12 , which corresponds to a 1.5-fold increase of 
entirely galactosylated proteins.

Univariate analysis of the non-targeted metabolite profiling results drew the attention to a number of intra- and extracellular metabolites having similar profiles than the extracellular lactate concentration. Interestingly, high levels of asparagine and homocysteine, for instance, were detected at the beginning of the culture, correlating with lactate production. Their levels eventually decreased when the cultured cells switched over to lactate consumption. Particularly, process A stood out with low levels of Asn and homocysteine, as well as a fast lactate concentration decrease starting on day 3. The lactate production peaks beyond day 10 were also correlated with increased levels of Asn and homocysteine. Processes B and D featured all at once the highest lactate concentration increases and the highest levels of the two amino acids. Homocysteine is either transformed to methionine (Met) by the Met-synthase using 5 -methyltetrahydrofolate or to cystathionine by the cystathionine- $\beta$-synthase, involving serine ${ }^{299}$. The non-targeted metabolomic analysis detected neither intra- nor extracellular cystathionine. On the other hand, Met did not display a similar pattern to the extracellular lactate concentration (data not shown).

The non-targeted profiling enabled to have a closer look at the relation of a number of substrates and their precursors of glycosylation enzymes and the resulting glycan pattern. The process format influenced the UDP-GlcNAc profile. In particular, process A exhibited lower levels in the second part of the fed-batch culture. However, the overall sum of agalactosylated, mono-galactosylated and di-galactosylated glycans remained comparable throughout the entire experiment and the A2 levels were small and did not differ substantially between the process formats (data not shown). It is assumed that the level of the GlcNAc transferase II substrate, UDP-GlcNAc, may have played a minor role at that stage of the glycan maturation. One explanation may be that the enzyme expression and the enzymatic reaction rate took precedence over the substrate availability. The cell might have compensated the differences in the intracellular UDP-GlcNAc levels by regulating genetic parameters and the presence of certain metabolites might have increased the enzyme activtiy. There is also evidence that ammonia accumulation correlated with enhanced UDP-GlcNAc formation as reported earlier ${ }^{141}$. This observation is at least applicable to processes $\mathrm{B}, \mathrm{C}$ and $\mathrm{D}$, which all exhibited higher extracellular ammonium and intracellular UDP-GlcNAc levels than process A. In contrast, more abundant intracellular UDP-glucose coincided with higher galactosylation. The enzyme UDP-galactose 4'-epimerase interconverts UDP-glucose and UDP-galactose in the final step of the Leloir pathway of galactose metabolism, while galactose-1-phosphate uridyl transferase is involved in the second step of the pathway, catalyzing the transformation of UDP-glucose and galactose-1-phosphate to glucose-1-phosphate and UDP-galactose ${ }^{124,302}$. In comparison to other process formats, in process A the substrate demand of galactosyltransferase was lower after day 6 . The lower degree of galactosylation at that time of the culture increases the substrate demand in order to reach a comparable percentage of galactosylated mAb to B, C and $\mathrm{D}$ at the end. It has been reported that despite 5 -fold higher intracellular UDP-galactose levels, galactosylation was not significantly improved ${ }^{123}$, which highlights the importance to take enzyme activity and gene expression into account too. The cofactor for galactosyltransferase, 
manganese, increases the GalT activity and thus considerably promotes galactosylation ${ }^{23,52}$. The three manganese containing processes $\mathrm{B}, \mathrm{C}$ and $\mathrm{D}$ exhibited improved galactosylation in particular around day 6 , and the presence of the trace element likely coincided with enhanced enzyme activity. Since ammonium continued to build up, the intra-Golgi pH increased, and as a result, increasingly hampered GalT activity despite Mn enhancement. Galactosylation drastically diminished, amounting to a maximum absolute difference of $-31 \%$ between the peak value and the endpoint. Media optimization might be considered to reduce detrimental effects of ammonium. An earlier study depicted how threonine, proline, and glycine had significant protective impact on important metabolic parameters, including glucose consumption, lactate production, glutamine utilization, and final ammonium levels ${ }^{301}$. At the same time an increase in the levels of sialic acid and galactosylated proteins was observed, which may indicate that the co-metabolism of those amino acids had possibly circumvented the detrimental effects of ammonia ${ }^{24,301}$. They also concluded that alanine secretion may reduce ammonium stress by bifurcation of the amine group from glutamate to alanine rather than in the form of a free ammonia molecule ${ }^{301}$. This finding suggests that the distinct alanine profiles in the present study may be a result of the high ammonium generating metabolisms of processes $\mathrm{B}, \mathrm{C}$ and $\mathrm{D}$.

As described above, many pathways were affected by changing the medium and feed composition. It is strenuous to analyze the plethora of intra- and extracellular results simultaneously and to draw meaningful conclusions by simple univariate analysis. Hence, multivariate analysis was performed to reduce the complexity of the data analysis in order to identify metabolites that directly or indirectly played a key role in the glycosylation pathways. Although the extracellular levels did not always reflect the intracellular levels, and more importantly, the trends, the PLS observation model based on the extracellular metabolites of the calibration set (processes A, B and D) proved to be reliable. The model predicted the level of mannose 5, afucosylated and fucosylated forms as well as the level of galactosylation of the prediction set (process C). Likewise, the following permutations leading to three additional models highlight that a high quality of prediction was obtained by all four combinations. The analysis of the model errors for each specific glycan species (RMSEE, RMSECV and RMSEP) shows that the models fairly well translate exometabolomic data into the glycan pattern at that specific time point. The major glycan species exhibited small errors. The errors were in the same range and lower than the real glycan values of the respective prediction set. Nonetheless, the prediction accuracy was substantially lower for minor glycan forms. This study also highlights the limitations of metabolomic profiling and the use of mathematical models explaining the relationship between metabolite levels and glycosylation patterns. Even if the PLS observation model explains the glycosylation pattern of the prediction set well, in particular on day 14 , it is certainly limited by the small number of batches included, and more importantly, by the absence of data regarding enzyme activity, expression levels, and substrate transport. The univariate analysis of the UDP-GlcNAc clearly reveals the limited capability of metabolomic profiling alone to explain certain glycan pattern characteristics. Glycosylation pathways are highly complex and involve multiple parameters. Despite the great diversity of extracellular metabolite profiles 
on day 6, the model error was substantially higher than for late culture time points. Further calibration with future development and commercial runs would increase the model quality. Despite these limitations, PLS-modelling of extracellular metabolomic data demonstrated its potential to predict glycosylation patterns. In large-scale manufacturing particularly, the measurement of extracellular metabolites may be easily and quickly carried out at-line or even on-line, using for instance Raman-based multivariate calibration models ${ }^{303}$. Real-time data rather than off-line analytical testing would be a great benefit in routine manufacturing for both monitoring and control. Thus, in future development efforts, it would be worthwhile and of great interest to further reduce the model complexity by including a small number of important variables only. For tight glycan pattern control a simplified model would be of great benefit to monitor critical cell culture process parameters on-line rather than relying on post-production interpretation of analytical results. This model can then be further calibrated with real large-scale data of future commercial batches.

\subsection{Conclusion}

Non-targeted endo- and exometabolomic profiling of cell culture processes offers great insights in the various pathways. As a starting point, the univariate analysis of the immense data sets pinpointed metabolites that featured a similar pattern than the distinct extracellular lactate profile of the four fed-batch processes. The degree of ammonium production and the presence of certain medium and feed components, namely manganese, influenced the time course of the glycosylation profile. Substrate or substrate precursors of the oligosaccharide transferases may be linked with the abundance of the corresponding glycan species. Nonetheless, the data suggests that the underlying processes are more complex and one should include further parameters, including enzyme activity, substrate transport and gene expression. In addition to reduced complexity due to the multivariate analysis of non-targeted metabolomic data, PLS modelling proved to be a suitable tool to investigate the correlations between metabolite levels and the glycan fingerprint. The resulting PLS observation model shows its great potential for the glycosylation control in routine manufacturing. 


\section{Chapter 9}

\section{Generation of a Wide Range of Glycan Variants for Bioactivity Testing}

\subsection{Introduction}

The structural features of recombinant therapeutic proteins shape their inherent biological activity in vivo ${ }^{11}$. The literature describes several examples linking glycosylation and biological activity. Glycosylation is essential to preserving its interaction with effector systems. Aglycosylated chimeric-mouse human IgG has for instance been reported to lose its binding capacity to the human $\mathrm{Fc} \gamma$-receptor $(\mathrm{Fc} \gamma \mathrm{RI})^{15}$. Sialic acid variants of a fusion protein (FST$\Delta$-Fc), namely the glycosylation sites located in the FST1 domain (sialylation was low at Fc domain), affected pharmacokinetic parameters in ASPGR-1 knockout mice, reducing clearing at higher level of sialylation ${ }^{304}$. Also in one case, its has been reported that higher levels of sialylated forms of IgG1 molecules have diminished antibody-dependent cellular cytotoxicity (ADCC) potency as a result of lower binding affinity to Fc $\gamma$ RIIIa ${ }^{198}$. Higher levels of sialylation of IgG brought about anti-inflammatory properties due to somewhat reduced binding to Fc $\gamma$ Rs $^{305}$. Many authors have described the pivotal role of Fc $\gamma$ RIII in connection with the efficacy of therapeutics ${ }^{67,306,307}$. It is not surprising that increasing effector functions by means of Fc-glycosylation engineering has gained considerable interest ${ }^{196}$. As a consequence, the pharmaceutical industry has allocated remarkable research efforts to glycan engineering, aiming to increase ADCC of mAbs, in particular by limiting core fucosylation ${ }^{48,65,91,308-313}$. Many reports have described the significant increase of ADCC whenever the fucose attachment to the glycan backbone was precluded ${ }^{42,196,308,312}$.

Health agencies may require an assessment of the impact on the biological activity, due to glycan pattern tuning, in the registration process of a new molecule. Easy and powerful ways to generate much wider intervals of glycoforms are needed so that the analytical methods may detect the induced changes on the biological activity. Following the example of the bio- 
therapeutics process development, media optimization stands out due to its ease of use and great potential. For instance, a simple and rapid method to produce non-fucosylated high mannose forms was proposed to assess biological activity of an IgG1, using kifunensine, a strong mannosidase I inhibitor ${ }^{91}$. Likewise, the addition of both potent and specific inhibitors of fucosyl- and sialyltransferases into cell culture media gave rise to complete enzyme inhibition $^{112}$. In this work, the effects of cell culture media supplements were levered by extending the glycosylation variation ranges as far as cell culture processes allow it in order to create a variety of mAb variants. Moreover, the goal was to test the performance of these glycosylation modulating compounds in the production of an antibody Fc-fusion molecule that contains three $\mathrm{N}$-glycosylation sites. One is located in the $\mathrm{CH} 2$ region of the Fc part of the antibody fraction, and the two other sites on the fusion entity (non-Fc part). As an alternative approach, the potential of enzymatic glycoengineering of purified proteins was assessed to further propel the levels of galactosylation and to remove sialic acid of the highly sialylated fusion molecule. Glycopeptide mass spectroscopy technology enabled to study the induced changes of the media supplements at each individual glycosylation site separately. The glycovariants were submitted to potency and binding assays. The results show that media supplementation allows to generate sufficient glycosylation variation for both classical mAb and complex molecules having several glycosylation sites, such as an antibody fusion molecule, and most importantly, statistically significant biological activity differences.

\subsection{Materials and Methods}

\subsubsection{Inoculum Preparation}

Two CHO-S derived clonal cell lines were used. Cell line A expressed a human monoclonal IgG1 antibody and cell line B an antibody Fc-fusion molecule comprised of three $\mathrm{N}$-glycosylation sites. Cells were first expanded in multiple passages in shake tubes or shake bottles in proprietary medium containing methionine sulfoximine (MSX) for at least 14 days in a shaker incubator at $36.5{ }^{\circ} \mathrm{C}, 5 \% \mathrm{CO}_{2}, 80 \%$ humidity and $320 \mathrm{rpm}$ agitation (ISF1-X, Adolf Kühner, Birsfelden, Switzerland or Multitron Cell, Infors HT, Bottmingen, Switzerland).

\subsubsection{Cell Culture}

Exponentially growing cells were seeded into a TPP® TubeSpin bioreactor tubes (referred to shake tubes or $S T$ ) filled with proprietary medium in the absence of MSX at a viable cell density of $0.30 \times 10^{6}$ viable cells $/ \mathrm{mL}$ for cell line A and $0.20 \times 10^{6}$ viable cells $/ \mathrm{mL}$ for cell line B. The supplements inducing a change of the glycosylation profile were added into the cell culture medium prior to seeding according to table 9.1. Ammonium chloride was also added on day 5 to reach $10 \mathrm{mM}$ in the cell culture solution. Depending on the nature of the supplement, different $\mathrm{NaCl}$ concentrations were added to medium to reach the same osmolality in all 
cultures. The tubes were incubated in a shaker incubator at $36.5^{\circ} \mathrm{C}, 5 \% \mathrm{CO}_{2}, 80 \%$ humidity and $320 \mathrm{rpm}$ agitation (ISF1-X, Adolf Kühner, Birsfelden, Switzerland) for 14 days (cell line A) and for 12 days (cell line B). Chemically defined feed containing over 30 components and alkaline amino acid solution were added on day 3, 5, 7 and 10. The $400 \mathrm{~g} / \mathrm{L}$ glucose solution was added the same days as well, and for cell line A at day 12 as well. Prior to each feeding and at the end of the culture (day 14), aliquots $(\leq 2.5 \mathrm{~mL}$ ) were taken for viable cell counting, extracellular metabolite profiling and product titer determination.

Table 9.1 - Medium supplement concentrations prior to inoculation.

\begin{tabular}{lll}
\hline Medium Supplement & Concentration & Supplier \\
\hline Raffinose & $65 \mathrm{mM}$ & Sigma \\
Kifunensine & $1 \mu \mathrm{M}^{\mathrm{a}}, 30 \mu \mathrm{M}^{\mathrm{b}}$ & Sigma \\
2F-peracetyl-fucose & $120 \mu \mathrm{M}$ & Merck \\
Manganese & $0.5 \mu \mathrm{M}$ & Merck \\
Galactose & $50 \mathrm{mM}$ & Merck \\
Ammonium chloride & $3 \mathrm{mM}^{\mathrm{c}}$ & Merck \\
\hline${ }^{\mathrm{a}}$ Cell lines A and B. & & \\
${ }^{\mathrm{b}}$ Cell line B. & & \\
${ }^{\mathrm{c}} 3$ mM NH $\mathrm{m}_{4}$ Cl prior to inoculation. Further $\mathrm{NH}_{4} \mathrm{Cl}$ \\
\multicolumn{2}{c}{ supplementation on day 5 to reach $10 \mathrm{mM}}$.
\end{tabular}

\subsubsection{Enzymatic Glycoengineering}

To evaluate the potential of enzymatic glycoengineering, drug substance (cell line A) and Protein A eluates (cell line B) were treated with $\beta$-1,4-galactosyltransferase (for cell line A, Sigma-Aldrich, Darmstadt, Germany; for cell line B, Roche, Basel, Switzerland) in $100 \mathrm{mM}$ MES (Sigma-Aldrich, Darmstadt, Germany) containing $10 \mathrm{mM}$ UDP-galactose (Sigma-Aldrich, Darmstadt, Germany). The protein was incubated during 4 hours at $37^{\circ} \mathrm{C}$ and $\mathrm{pH} 6.5$. In a separate set of experiments, Protein A eluates of cell line B were incubated in $75 \mathrm{mM}$ sodium phosphate (Prozyme, Hayward CA, USA) at pH 6.0 in the presence of Sialidase ATM (Prozyme, Hayward CA, USA) for 1 hour at $37^{\circ} \mathrm{C}$.

\subsubsection{Analytical Methods for Cell Culture Performance}

Growth and viability assessment was performed using a Vi-Cell analyzer (Beckman Coulter, Brea, CA). The product titer of the shake tubes collected on days 5, 7, 10, 12 (cell lines 1 \& 2), and day 14 (cell line A) was performed by a Biacore C instrument (GE Healthcare, Waukesha, WI) for cell line A and by Protein A HPLC for cell line B. 


\subsubsection{Glycan Analysis}

Cell line A expresses an antibody having one glycosylation site at the Fc part of the molecule (CH2 domain). The antibody fusion molecule of cell line B has three different glycosylation sites. One is located at the Fc region of the antibody part and two distinct sites are found on the fusion entity (non-Fc part) as shown in table 9.2.

Table 9.2 - Glycan sites of antibody (cell line A) and fusion antibody (cell line B).

\begin{tabular}{lll}
\hline Cell line & Glycan location & Label \\
\hline A & Fc part & - \\
B & Fc part & Site 1 \\
& Fusion entity & Site 2 \\
& Fusion entity & Site 3 \\
\hline
\end{tabular}

The cell culture supernatants and the treated enzymatic glycoengineering samples of cell line A were loaded onto a pre-packed ProSep ${ }^{\circledR}$ Ultra Plus column $(1 \mathrm{~mL}, 0.8 \times 2 \mathrm{~cm}$, Merck Life Science, Darmstadt, Germany). Subsequent elution in $20 \mathrm{mM}$ acetic acid at pH 4.0, $1 \mathrm{mM}$ MES was added to reach a final $\mathrm{pH}$ of 5.2. The neutralized samples were denatured by IAA in $0.6 \mathrm{M}$ denaturation reagent (GlykoPrep-plus, Europa Bioproducts, Cambridge, UK) and reduced. Following purification, the samples were labelled with 2-amino-benzamide and then dried for 3 days. The dried samples were dissolved in $50 \% \mathrm{ACN}$ and subsequently injected into the $100 \mathrm{~mm}$ UPLC column in length supplied by Waters Corporation, Milford, MA, USA and eluted, using a gradient. The individual glycans were grouped according to table 9.3 for enhanced visual interpretation of the data presented hereafter. For cell line B, the supernatants and the glycoengineering samples were purified by a ProSep ${ }^{\circledR}$ Ultra Plus column $(0.6 \mathrm{~mL}, 0.5 \times$ $3 \mathrm{~cm}$, Merck Life Science, Darmstadt, Germany) and eluted with $20 \mathrm{mM}$ acetic acid at pH 3.2. Subsequent $\mathrm{pH}$ adjustment with $1 \mathrm{M}$ Tris to $\mathrm{pH}$ 5.0, the samples were suspended in the denaturation buffer containing $8 \mathrm{M}$ guanidine- $\mathrm{HCl}, 1 \mathrm{mM}$ EDTA, $130 \mathrm{mM}$ Tris $\mathrm{pH}$ 7.6. Reduction was performed at $37^{\circ} \mathrm{C}$ by DTT. Then, alkylation was performed in dark, at room temperature for 30 minutes, with IAM. The reduced and alkylated samples were buffer exchanged with the digestion buffer containing $2 \mathrm{M}$ urea, $50 \mathrm{mM}$ Tris- $\mathrm{HCl}, \mathrm{pH} 8.0$ using Amicon Ultracel 10K centrifugal filters. The protein was digested with trypsin with an enzyme-to-substrate ratio of $1: 20$, at $37^{\circ} \mathrm{C}$ for 4 hours and subsequently analyzed by Xevo G2-S, Q-TOF Mass Spectrometer (Waters, Milford, MA) equipped with an Acquity UPLC system (Waters, Milford, MA). The peptides were separated on an Acquity BEH glycan UPLC column $(1.7 \mu \mathrm{m}, 2.1 \times 150 \mathrm{~mm}$, Waters, Milford, MA). The solvents used for the analysis were A: $10 \mathrm{mM}$ ammonium formate pH 4.5, and B: $10 \mathrm{mM}$ ammonium formate in $90 \%$ acetonitrile. MS and MSE spectra were acquired in the data-independent mode by alternating the high and low energy values for collision-induced dissociation (CID). The instrument was operated with a capillary voltage of $3 \mathrm{kV}$, sampling cone of 37 , source temperature of $100^{\circ} \mathrm{C}$, desolvation temperature of $250^{\circ} \mathrm{C}$, cone gas flow of $10 \mathrm{~L} / \mathrm{h}$, desolvation gas flow of $500 \mathrm{~L} / \mathrm{h}$, and a scan range of $100-2500 \mathrm{~m} / \mathrm{z}$. Data processing was performed by BiopharmaLynx 1.3.4 software (Waters, Milford, MA). The 
values for the relative distribution of the glycans at each of the $\mathrm{N}$-glycosylation sites were computed by the software. The data were manually checked for the false positives and/or miss-assignments. The individual glycans were grouped according to table 9.4 for enhanced visual interpretation of the data presented hereafter.

Table 9.3 - Glycan grouping for cell line A.

\begin{tabular}{ll}
\hline Glycan & Sum of glycan peaks \\
\hline $\mathrm{HM}=$ & $\mathrm{M} 4+\mathrm{M} 5+\mathrm{M} 6+\mathrm{M} 7+\mathrm{M} 8+\mathrm{M} 9$ \\
$\mathrm{AF}=$ & $\mathrm{A} 0+\mathrm{A} 1+\mathrm{A} 2$ \\
$\mathrm{Fuc}=$ & $\mathrm{FA} 1+\mathrm{FA} 2$ \\
$\mathrm{Gal}=$ & FA2G1+FA2G2+FA3G1+(FA1[3]G1+A2[6]G1) \\
& $+(\mathrm{A} 2[3] \mathrm{G} 1+\mathrm{M} 5 \mathrm{~A} 1)+\mathrm{A} 2 \mathrm{G} 2)$ \\
Sial $=$ & FA2G2S1+A2G2S1+A2G2S2+(FA2G2S2+FA2G2S1(NGNA)) \\
Misc $=$ & UKN_1.834+Hybrid-F+FA2BG1+FM5A1G1S1 \\
\hline
\end{tabular}

\subsubsection{Analysis of Biological Activity}

\section{Cell Line A}

FcrRIII affinity (SPR)—Real-time biomolecular interaction analysis (BIA) uses the optical phenomenon called surface plasmon resonance (SPR) to monitor biomolecular interaction. Detection depends on changes in the mass concentration of macromolecules at the biospecific interface and requires no labelling of interactants. Interactions are followed in real time, so that kinetic information is readily derived. Measurements with Biacore are based on the interaction of the analyte ( $\mathrm{mAb}$ ) in solution with the ligand (Fcg $\gamma \mathrm{RIII})$ immobilized to a sensorchip. The sensor-chip is a glass slide coated with a thin layer of gold, to which a matrix of carboxymethylated dextran is covalently attached. The gold is required for the generation of the surface plasmon resonance (SPR) response. A light source is focalized on the sensor-chip by a prism with a specific angle of incidence and the reflected light is revealed by a detector array. The binding of the analyte to the chip modifies the angle of incidence generating variations of the SPR. The association and dissociation kinetic rates $\left(k_{a}\right.$ and $\left.k_{d}\right)$ and the deduced dissociation affinity constant $\left(\mathrm{K}_{\mathrm{D}}=\mathrm{k}_{\mathrm{d}} / \mathrm{k}_{\mathrm{a}}\right)$ of the cell line A product samples were determined, using Biacore SPR technology. While the Fc-receptor proteins were captured on an immobilized Tetra.His Antibody on a sensor-chip, the signal of mAb binding at several different concentrations complexed with the target ligand was measured in real time for binding to the low affinity Fc $\gamma$ RIIIa (V158 and F158 isoforms) and IIIb receptors. The mean affinities of the mAbs for FcgRIIIs were expressed as $\mathrm{K}_{\mathrm{D}}(\mu \mathrm{M})$ obtained from 3 independent experiments.

ADCC reporter activity (Luminescence)—Cell line A product capability to induce ADCC pathway activation was determined, using the ADCC Reporter Bioassay (Promega). This bioluminescent 
Table 9.4 - Glycan grouping for cell line B.

\begin{tabular}{|c|c|c|c|}
\hline Site & Glycan & & Sum of glycan peaks \\
\hline \multirow[t]{6}{*}{1} & HM & $=$ & $\mathrm{M} 5+\mathrm{M} 6+\mathrm{M} 7+\mathrm{M} 8+\mathrm{M} 9$ \\
\hline & $\mathrm{AF}$ & $=$ & $\mathrm{A} 1+\mathrm{A} 2$ \\
\hline & Fuc & $=$ & $\mathrm{FA} 1+\mathrm{FA} 2$ \\
\hline & Gal & $=$ & $\mathrm{A} 2 \mathrm{G} 1+\mathrm{A} 2 \mathrm{G} 2+\mathrm{FA} 2 \mathrm{G} 1+\mathrm{FA} 2 \mathrm{G} 2$ \\
\hline & Sial & $=$ & FA1G1S1 \\
\hline & Misc & $=$ & FM4A1G1 \\
\hline \multirow[t]{6}{*}{2} & HM & $=$ & $\mathrm{M} 4+\mathrm{M} 5+\mathrm{M} 6+\mathrm{M} 7+\mathrm{M} 8+\mathrm{M} 9$ \\
\hline & $\mathrm{AF}$ & $=$ & $\mathrm{A} 2+\mathrm{A} 3$ \\
\hline & Fuc & $=$ & $\mathrm{FA} 1+\mathrm{FA} 2+\mathrm{FA} 3+\mathrm{FA} 4$ \\
\hline & Gal & $=$ & A2G2+FA2G1+FA2G2+FA3G1+FA3G2+FA3G3+FA4G4 \\
\hline & Sial & $=$ & $\begin{array}{l}\text { A2G1S1+A2G2S2+FA1G1S1+FA2G1S1+FA2G2S1_1Ac } \\
+ \text { FA2G2S1_2Ac+FA2G2S2+FA2G2S2_1Ac+FA2G2S2_1NGNA }\end{array}$ \\
\hline & Misc & $=$ & Not detected \\
\hline \multirow[t]{14}{*}{3} & $\mathrm{HM}$ & $=$ & $\mathrm{M} 5+\mathrm{M} 6+\mathrm{M} 7+\mathrm{M} 8+\mathrm{M} 9$ \\
\hline & $\mathrm{AF}$ & $=$ & A3 \\
\hline & Fuc & $=$ & $\mathrm{FA} 2+\mathrm{FA} 3+\mathrm{FA} 4$ \\
\hline & Gal & $=$ & FA3G1+FA3G2+FA3G3+FA4G1+FA4G4+FA4G4L1 \\
\hline & Sial & $=$ & A3G1S1+A3G2S2+A3G3S2+A3G3S3+A3G3S3_1Ac \\
\hline & & & +A4G3S2+A4G3S3+A4G4S2+A4G4S3+A4G4S4+A4G4S4_1Ac \\
\hline & & & +FA1G1S1+FA2G1S1+FA2G2S1+FA2G2S2+FA2G2S2_1Ac \\
\hline & & & +FA3G1S1+FA3G2S1+FA3G2S2+FA3G3S1+FA3G3S2+FA3G3S2_1Ac \\
\hline & & & +FA3G3S3+FA3G3S3_1Ac+FA3G3S3_1NGNA+FA3G3S3_2Ac \\
\hline & & & +FA4G1S1+FA4G3S1+FA4G3S2+FA4G3S3+FA4G4L1S2 \\
\hline & & & +FA4G4L1S3+FA4G4L1S4+FA4G4S1+FA4G4S2+FA4G4S2_1Ac \\
\hline & & & +FA4G4S3+FA4G4S3_1Ac+FA4G4S3_1NGNA+FA4G4S4 \\
\hline & & & +FA4G4S4_1Ac+FA4G4S4_1NGNA+FA4G4S4_2Ac \\
\hline & Misc & $=$ & FM4A1G1 \\
\hline
\end{tabular}


reporter assay quantified pathway activation by antibodies on gene transcription in effector cells stably expressing the Fc $\gamma$ RIIIA receptor, V158 (high affinity) variant, and a NFAT response element driving expression of firefly luciferase. Antibody biological activity in ADCC reporter bioassay was quantified with a luminescence readout of the luciferase produced in the effector cells, as a result of NFAT pathway activation induced by crosslinking with the antibody and the target cells. The ADCC reporter bioassay was performed according to manufacturer instructions. Cell line A product was serially diluted and incubated with effector and target cells at an effector-to-target ratio ( $\mathrm{E}: \mathrm{T}$ ratio) of $6: 1$. Finally, luminescence was read after the addition of the luminescent substrate, using a plate reader with a glow luminescence detector. The fold induction was calculated with respect to the luminescence produced without antibody. The experimental data were interpolated with the $4 \mathrm{PL}$ algorithm and the half-maximal effector concentration $\left(\mathrm{EC}_{50}\right)$ of luciferase activity (fold induction) was obtained and expressed as percentage of activity relative to the untreated control material. The ADCC reporter activity was released as average of results coming from three independent assays.

Clq binding (ELISA)—Cell line A product binding capability to the human Clq was determined by ELISA. Different concentrations of the mAb were coated onto a 96-well plate. Unbound sites were blocked with $1 \%$ BSA, then a fixed concentration of Clq was added to the mAb coated 96-well plate and allowed to react. The reaction was revealed by means of addition of anti Clq-HRP antibody and a proper substrate that triggered a colorimetric reaction. The intensity of the colorimetric signal was directly proportional to the C1q protein bound to the coated antibody. The experimental data were interpolated with the 4PL algorithm. The Clq binding activity of a sample was expressed as percentage of activity with respect to the untreated control material and was the percentage expression of the half-maximal effector concentration $\left(\mathrm{EC}_{50}\right)$. The $\mathrm{Clq}$ binding activity was released as average of results coming from three independent assays.

CDC activity (Luminescence)—Cell line A product capability to induce Clq pathway activation was determined, using a CDC assay. Target-cells were incubated in a dilution series of the untreated control material or cell line A product and rabbit serum was added to the wells as a source of complement. Following incubation, the reduction of target cell viability was determined by a luminescent cell viability assay measuring ATP levels. The data was fitted to a 4PL model and the half-maximal effector concentration $\left(\mathrm{EC}_{50}\right)$ was reported relative to the untreated control material response. The $\mathrm{CDC}$ reporter activity was released as average of results coming from two independent assays.

\section{Cell Line B}

Reporter gene assay - The purified samples of cell line B were analyzed by both a reporter gene and ligand binding assay. For each reporter gene bioassay, 50,000 $4 \mathrm{~T} 1$ cells/well were loaded on a 96-well plate and allowed to adhere to the plate for 4 to 5 hours at $37^{\circ} \mathrm{C}, 5 \% \mathrm{CO}_{2}$. Then, the cells were pre-coated on the plate. The dose-response curves for both reference standard 
and samples were prepared (from 100 to $0.78125 \mathrm{ng} / \mathrm{mL}$ ) and added to the pre-coated plates. Subsequently, a fixed amount of $5 \mathrm{ng} / \mathrm{mL}$ of the recombinant human immune suppressor protein was added. The plate was incubated overnight at $37^{\circ} \mathrm{C}, 5 \% \mathrm{CO}_{2}$. Luciferase expression was evaluated by using D-Luciferin reagent. When luciferin is oxidized under the catalytic effects of luciferase and ATP, a bluish-green light is produced. The emitted light is inversely related to the protein concentration. The output was plotted against the log transformed protein concentration and fitted by 4PL. For each data set, the protein concentration able to inhibit $50 \%$ of the maximum possible $\left(\mathrm{EC}_{50}\right)$ was automatically calculated. The biological activity of a sample was expressed as percentage of activity with respect to the reference material and derived from the percentage expression of the potency ratio. The potency was released as average of results coming from three independent assays.

Ligand binding assay - In the frame of the ligand binding assay, the capability to bind to the receptor was quantified. Microtiter plates (MaxiSorp Plate for ELISA) were coated with Protein A ( $5 \mu \mathrm{g} / \mathrm{mL})$ overnight at $5{ }^{\circ} \mathrm{C}$ or for 1 hour at room temperature. The plate was washed and blocked with a $1.5 \%$ BSA solution. Different concentrations of the antibody fusion molecule (from $600 \mathrm{ng} / \mathrm{mL}$ to $4.6875 \mathrm{ng} / \mathrm{mL}$ ) were coated onto the Protein A coated plate, through the binding of the antibody Fc portion, for 30 minutes at room temperature under swirling. Then $50,000 \mathrm{HEK}-293$ cells/well were added to the coated plate and allowed to bind to the receptor for 1 hour at $37^{\circ} \mathrm{C}, 5 \% \mathrm{CO}_{2}$. The unbound cells were then washed out and the bound cells revealed in each well by the ATPlite 1 step, an ATP monitoring system. The binding capability was released as average of results coming from three independent assays.

\subsection{Results}

\subsubsection{Cell Culture Performance}

Using the potential of the two cell lines with the aim to generate a large glycan diversity, high medium supplement concentrations were required. First, the interest was directed towards their effect on the cell culture performance including cell growth and productivity. According to figure 9.1A three groups of viable cell densities profiles of cell line A can be distinguished. Manganese and galactose as well as ammonium supplemented cultures displayed comparable viable cell density to the control cultures. 2F-peracetyl fucose addition entailed a comparable cell growth until the peak at day 7 before decreasing faster as the production phase progressed. Kifunensine and particularly raffinose supplementation inhibited cell growth. Medium supplementation had limited effects on the product titer at harvest (day 14). As one can note in figure 9.1C, the presence of kifunensine and ammonium led to a small titer decrease, while the other supplements did not impact product concentration. With the exception of raffinose and kifunensine, the growth of cell line B was not altered due to the presence of the quality modulators (figures 9.2A and B). While raffinose hampered growth, entailing a peak cell density of $12.3 \times$ $10^{6}$ viable cells $/ \mathrm{mL}$ (the non-supplemented control cultures of the first series maxed out at 
$19.1 \times 10^{6}$ viable cells $/ \mathrm{mL}$ ), $30 \mu \mathrm{M}$ kifunensine displayed a drastic effect on the cell density. These cultures reached a peak of $6.4 \times 10^{6}$ viable cells $/ \mathrm{mL}$ and the non-supplemented control cultures of the second series amounted to $16.9 \times 10^{6}$ viable cells $/ \mathrm{mL} .1 \mu \mathrm{M}$ kifunensine had no detrimental effect of growth. In contrast to cell line A, raffinose also lowered the product titer of cell line B (figure 9.2C). The control culture (first series) reached $1280 \mathrm{mg} / \mathrm{L}$ at harvest on day 12. In the presence of raffinose, the expressed amount decreased about twofold, coming to a halt at $720 \mathrm{mg} / \mathrm{L}$. With $1 \mu \mathrm{M}$ kifunensine the culture yielded comparable titers. On the other hand, the 30-fold higher kifunensine concentration reduced the product titer. The duplicate ST experiments yielded $692 \mathrm{mg} / \mathrm{L}$ on average, while the control of the second series contained $1236 \mathrm{mg} / \mathrm{L}$ of the protein of interest.

\subsubsection{Glycan Distribution}

Media supplementation of cell line A cultures produced a variety of distinct glycoforms (figure 9.3). Both raffinose and kifunensine increased high mannose glycans. They climbed up to $9.1 \%$ with raffinose. Kifunensine addition mainly resulted in mannosylated mAbs, amounting to $81 \%$, and thus, this experiment covered a range between $2.2 \%$ and $81 \%$ of high mannose. In the conditions of this study, the addition of $2 \mathrm{~F}$-peracetyl fucose pushed the afucosylated species from $1.8 \%$ up to $89.9 \%$. Raffinose and kifunensine decreased fucosylated species as a consequence of the increase of high mannoses, which are linked. Likewise, in the presence of 2F-peracetyl fucose, they diminished due to fucosyltransferase inhibition. Manganese and galactose led to more frequent docking of galactose onto terminal GlcNAc of FA2 glycan entities. In total, the mono-galactosylated form FA2G1 and the di-galactosylated FA2G2 peaked at $50.1 \%$ while the level of galactosylation in the control amounted to $13.8 \%$. Ammonium supplementation reduced terminal galactose to $6.8 \%$. By this means, cell culture medium supplementation enabled to vary the level of galactosylation, covering a range between $6.8 \%$ and $50.1 \%$. As anticipated, the supplements had minor ramifications on sialylation. The combined supplementation of manganese and galactose was also compared with the enzymatic glycoengineering of drug substance, using galactosyltransferase (GalT). Subsequent GalT treatment, $35.0 \%$ of the antibody was galactosylated. In comparison to GalT treatment, Mn \& Gal supplementation yielded a higher level of entirely galactosylated mAbs. Medium supplementation resulted in 40.2\% FA2G1 and 8.1\% FA2G2. Dissimilarly, GalT treatment produced $29.4 \%$ FA2G1 and 3.8\% FA2G2.

Analogously to cell line A cultures, the medium supplements produced a variety of glycan distributions in cell line B (figure 9.4). Nevertheless, each of the three N-glycosylation sites responded in distinct ways to the presence of the additives. In comparison with the important changes that kifunensine supplementation induced, the controls of series 1 and 2 were sufficiently similar, and hence, only the control of the first series will be presented as comparison hereafter. In the control, the FA2 species was predominant at site 1 located at the $\mathrm{CH} 2$ domain of the antibody backbone of the fusion protein. Raffinose supplementation slightly increased high mannose species. While $1 \mu \mathrm{M}$ kifunensine produced weak effects, this alkaloid strongly 


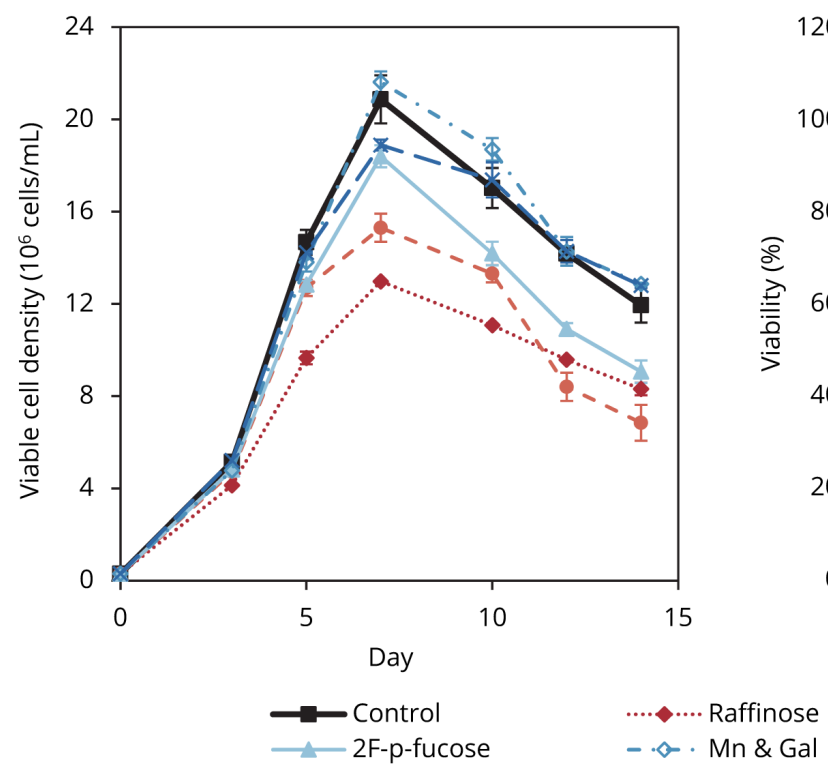

C

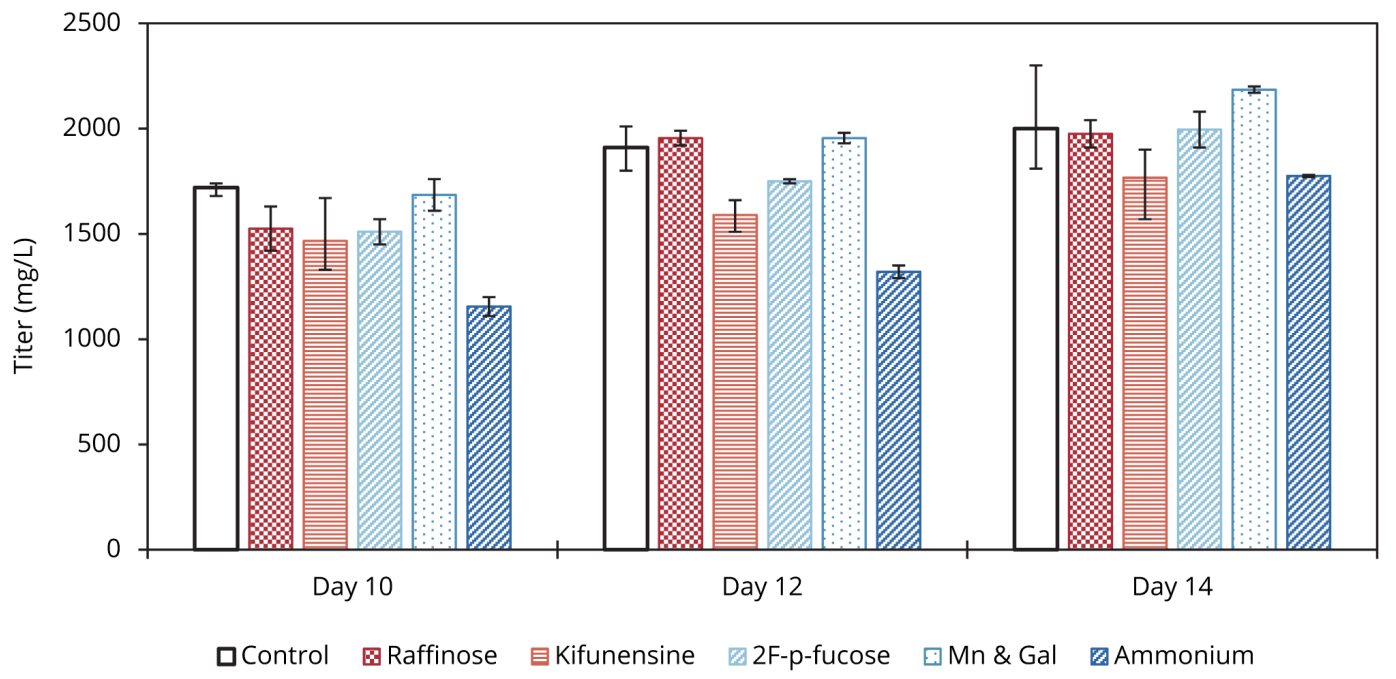

Figure 9.1 - (A) Viable cell densities of cell line A cultures supplemented with glycosylation modulators and non-supplemented control culture. The control and the kifunensine supplemented cultures were conducted in triplicates $(n=3)$, while the others in duplicates $(n=2)$. (B) Viabilities. (C) Product titers on days 10, 12 and 14. In all charts average values of the replicates are shown. The error bars indicate the upper and lower limits of the values. 
A

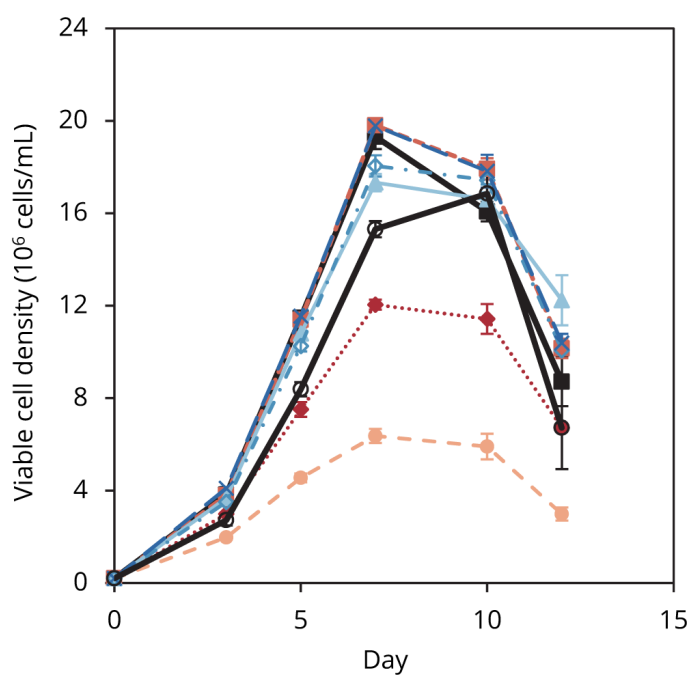

- - Kifunensine $30 \mu \mathrm{M}$

$-\star$ Ammonium

C

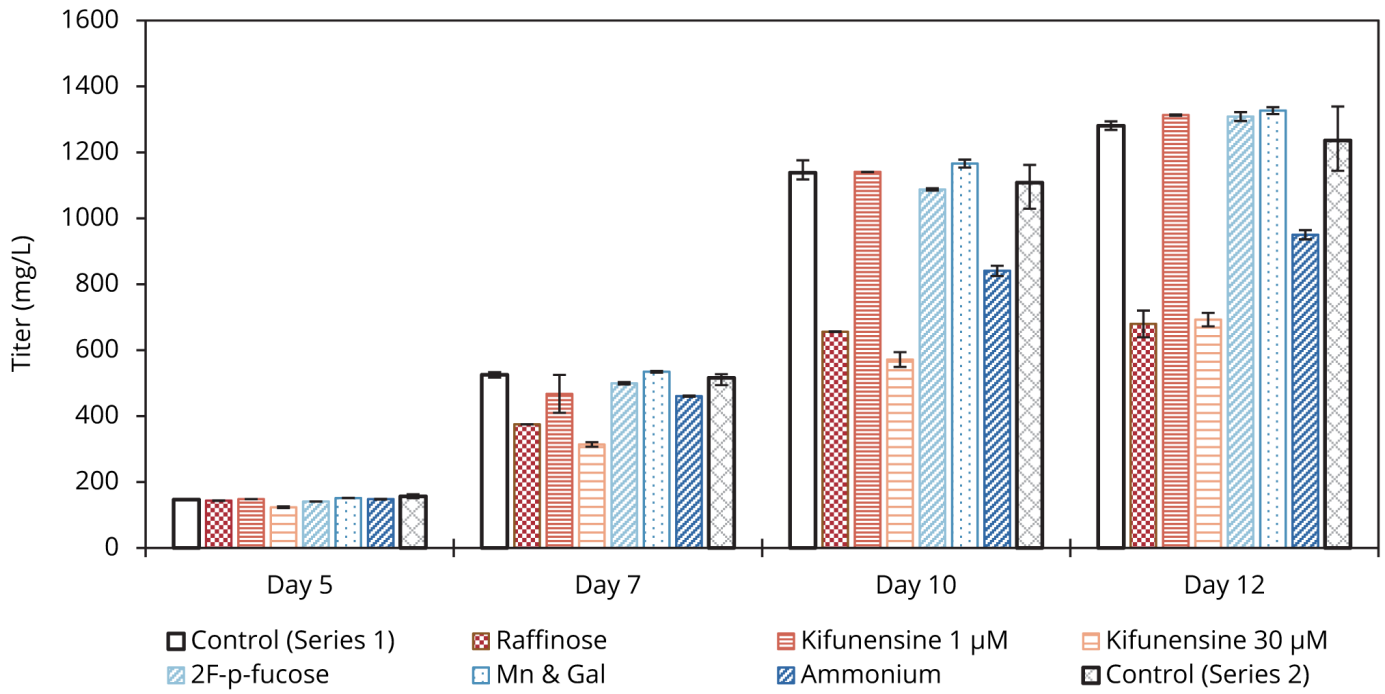

B

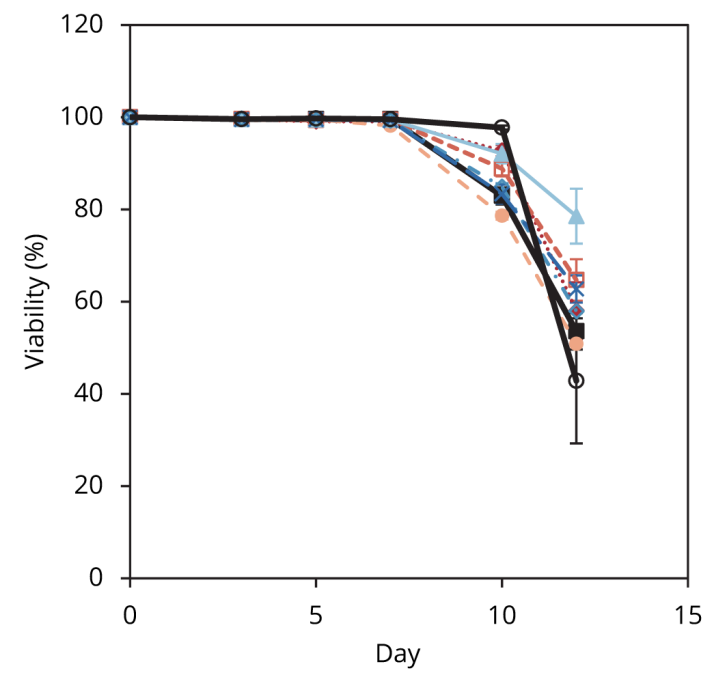

-- - Kifunensine $1 \mu \mathrm{M}$

$-\diamond \cdot \mathrm{Mn} \& \mathrm{Gal}$

Figure 9.2 - (A) Viable cell densities of cell line B cultures supplemented with glycosylation modulators and non-supplemented control culture. The experiment was conducted in two independent series. At the exception of $30 \mu \mathrm{M}$ kifunensine, the conditions belong to series 1 . Both control 1 and the control of the second series (control 2) were conducted in triplicates $(n=3)$. All supplemented cultures of series 1 and 2 were performed in duplicates $(n=2)$. (B) Viabilities. (C) Product titers on days 5, 7, 10 and 12. All charts show average values of the replicates. The error bars indicate the upper and lower limits of the replicate values. 


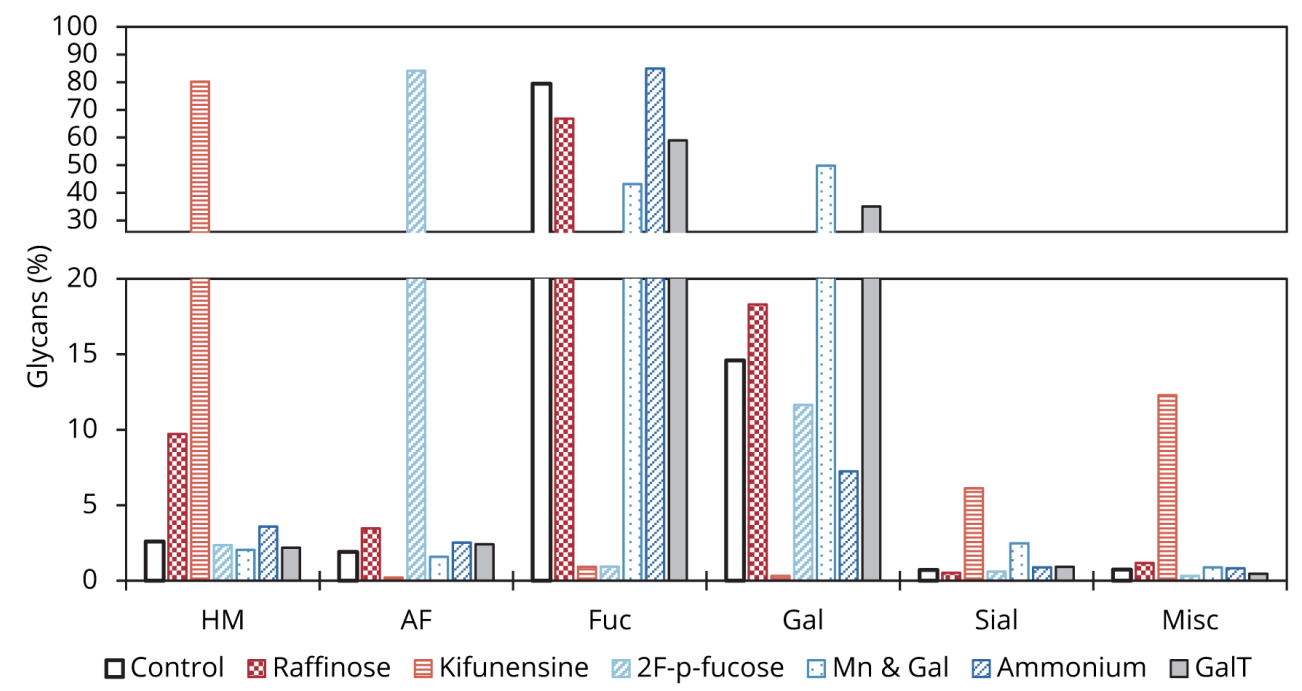

Figure 9.3 - Glycan pattern of control and cultures supplemented with either raffinose, kifunensine, 2F-p-fucose, manganese \& galactose, or ammonium (cell line A).

impacted the glycosylation pathway and thus predominantly generated high mannose glycans at $30 \mu \mathrm{M}$. Its presence raised the abundance of $\mathrm{HM}$ up to $99.7 \%$. 2F-p-fucose increased the afucosylated forms from 1.0 to $82.2 \%$. Manganese and galactose addition favored galactosylation (increase from 14.6 to $53.4 \%)$. Ammonium slightly limited terminal galactose (-2.9\%). Enzymatic treatment using GalT resulted in a surpassing degree of terminal galactose. It amounted to $98.6 \%$ and the FA2 peak completely vanished. While media supplementation mainly produced mono-galactosylated forms (FA2G1: 45.2\%, FA2G2: 8.2\%), complete galactosylation abounded ensuing enzymatic glycoengineering (FA2G1: 41.3\%, FA2G2: 57.3\%). In the control, terminal sialic acid reached $79.1 \%$ (site 2) and $80.9 \%$ (site 3). Like at the $\mathrm{CH} 2$ domain, $30 \mu \mathrm{M}$ kifunensine exhibited potent effects on the glycosylation maturation of sites 2 and 3. HM amounted to $98.7 \%$ at the former and $79.0 \%$ at the latter site. All other supplements did not produce important high mannose changes. At a first glance, the effect of 2F-p-fucose seemed to be weak. Its presence resulted in an increase of afucoslyated species of $16.0 \%$ (site 2) and $5.9 \%$ (site 3 ). The grouping of afucosylated species exclusively contained non-galactosylated and non-sialylated species, which was perfectly adapted to mAb glycans. In figure 9.5, the glycan grouping of afucosylated and fucosylated species was enhanced to obtain a suitable resolution for sites 2 and 3. In this chart, both afucosylated and fucosylated species encompass galactosylated and sialylated forms as well. 2F-p-fucose supplementation exhibited strong fucosylation inhibition at both fusion moiety sites. At site 2, afucosylated forms amounted to $78.5 \%$ (control: $0.2 \%$ ) and at site 3 , they reached $91.4 \%$ (control: none). At both fusion-moiety sites (figure 9.4), manganese and galactose supplementation reduced fucosylated structures, but the level of galactosylation remained unchanged. The greater availability of terminal galactose moieties seemed to favor sialylation that rose to $96.5 \%$ at site 2 (control: $79.1 \%$ ) and $96.6 \%$ at site 3 (control: $80.9 \%$ ). Interestingly, the presence of raffinose also enhanced sialylation at sites 2 and 3. The trisaccharide produced increases of 13.3 and $15.0 \%$ at sites 2 
and 3 , respectively. In comparison to site 1 , the fusion moiety glycosylation sites were more responsive to the ammonium level throughout the cell culture. Fucosylated species increased by $8.6 \%$ (site 2) and $13.1 \%$ (site 3). GalT treatment of the cell culture supernatant resulted in a reduction of the fucosylated species in favor of galactosylated species. They increased by $9.8 \%$ at site 2 and $3.1 \%$ at site 3 . Surprisingly, the level of sialic acid moieties ended up at a higher level $(+9.0 \%$ at site $2,+12.5 \%$ at site 3$)$. The treatment of the supernatant with sialidase was the only way to increase terminal galactose by means of enzymatic removal of terminal sialic acid. After completion of the enzymatic removal, $81.6 \%$ (site 2) and $78.8 \%$ (site 3) of the recombinant protein entities were galactosylated. At the same time sialylation was low, amounting to $2.6 \%$ at site 2 and $0.3 \%$ at site 3 .

Furthermore, the interest was directed towards the influence of the medium supplements and the enzymatic treatment on the antennarity of the generated glycans. 2F-p-fucose brought about an interesting variation. While the fucose analog did not feature any effect on the distribution between mono- and biatenary glycans at the $\mathrm{CH} 2$ domain site, particularly the sialylated species of sites 2 and 3 were strongly affected (figure 9.6). The biantennary sialylated forms of the control summed up to $41.9 \%$, the triantennary and tetraantennary forms to $34.0 \%$ and $3.2 \%$, respectively, at site 2 . In the $2 \mathrm{~F}$-p-supplemented culture, the majority of sialylated glycans were afucosylated and the triantennary forms were predominant (53.6\%). The biantennary forms decreased to $12.4 \%$ and tetrantennarity increased to $7.5 \%$. Similarly, in the presence of the fluorinated fucose compound, the triantennarity dropped to $13.9 \%$ (control: 39.6\%). The tetraantennary sialylated forms nearly doubled, reaching $76.6 \%$ (control: $39.5 \%$ ). Moreover, 2F-p-fucose generated comparable levels of the afucosylated entities A2 and A3 compared to the control culture fucosylated forms (FA2 and FA3). At both fusion moiety sites the equivalent abundance of FA4 in the control did not emerge in the afucosylated group. Rather than favoring the A4 from, the 2F-p-fucose supplementation presumably contributed to the increase of the tetrasialylated forms.

\subsubsection{Biological Activity}

The great choice of glycans that arouse following cell culture supplementation and enzymatic glycoengineering, using galactosyltransferase and sialidase, enabled to assess how the induced changes affected the biological activity in vitro. For cell line A, the resulting glycan distribution of raffinose, kifunensine and 2F-p-fucose supplemented cultures displayed significant effects on the affinities to the Fc $\gamma$ receptor III and on the ADCC reporter potency (figures 9.7A to $\mathrm{C}$ ). The binding affinity $\left(\mathrm{K}_{\mathrm{D}}\right)$ of $\mathrm{mAb}$ of the control culture with the Fc $\gamma$ RIIIa V158 receptor amounted to $0.6 \mu \mathrm{M}$. Raffinose supplementation brought about an increased affinity $\left(\mathrm{K}_{\mathrm{D}}=0.4 \mu \mathrm{M}\right)$. Both kifunensine and 2F-p-fucose effected great affinity increases with binding affinity constants of 0.1 and $0.002 \mu \mathrm{M}$, respectively. Likewise, the three supplements produced enhanced affinities for the Fc $\gamma$ RIIIa F158 receptor. The $K_{D}$ of the non-supplemented control culture reached $1.7 \mu \mathrm{M}$. The ranking was preserved and thereby the affinity constant of raffinose, kifunensine and 2F-p-fucose experiments equalled 1.0, 0.25 and $0.12 \mu \mathrm{M}$, respec- 


\section{A - Site 1}

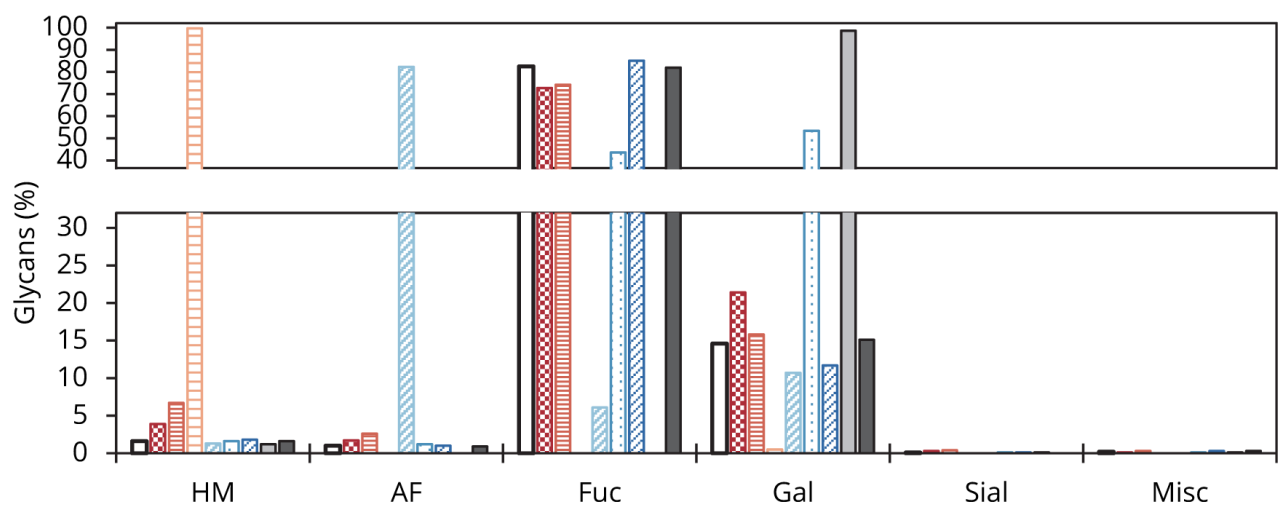

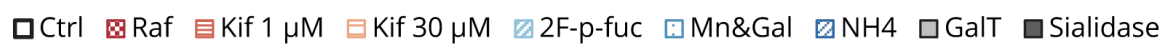

B - Site 2

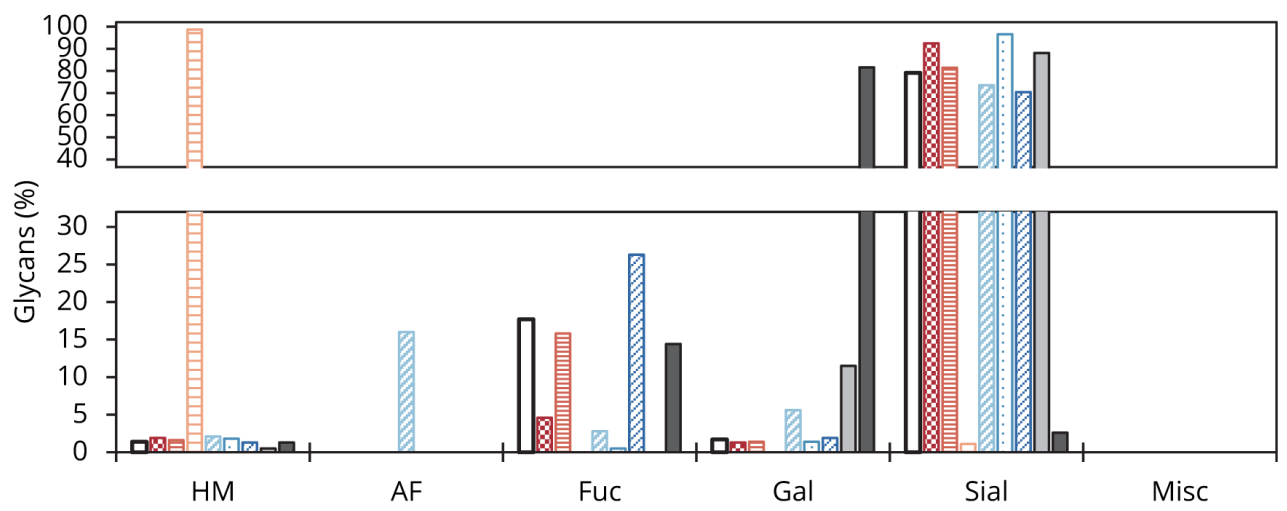

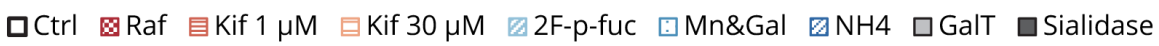

C - Site 3

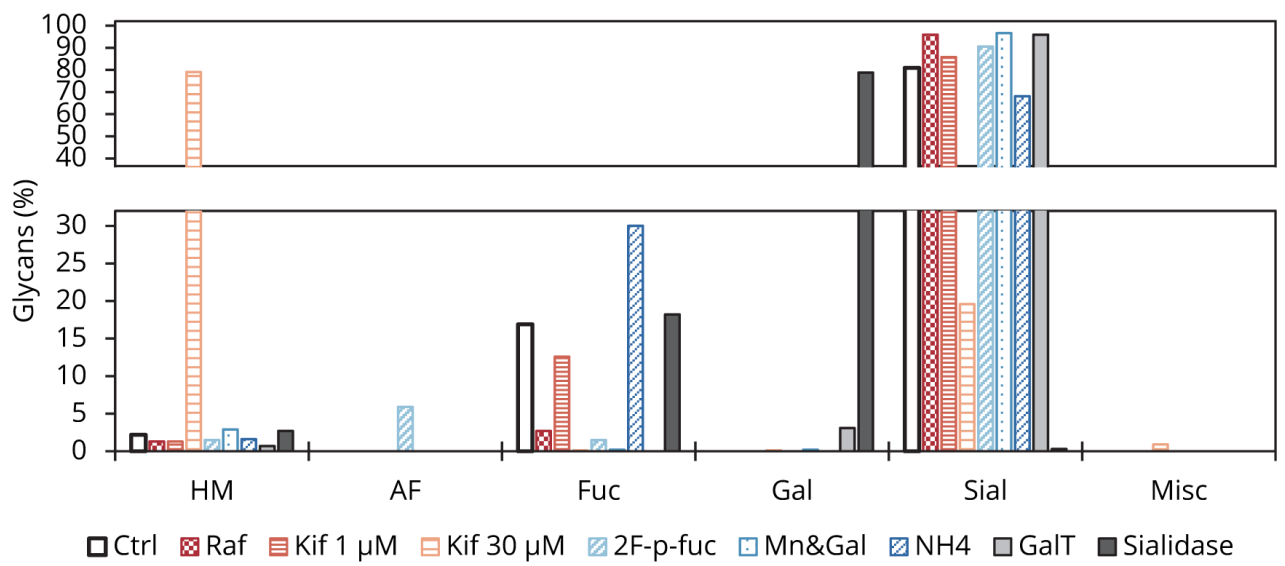

Figure 9.4 - Glycan pattern of control and cultures supplemented with either raffinose, kifunensine, 2F-p-fucose, manganese \& galactose, or ammonium (cell line B). Each chart corresponds to one of the three glycan sites. (A) Glycan site 1 located at Fc domain. (B) Glycan site 2 located at non-Fc-part of fusion entity. (C) Glycan site 3 located at non-Fc-part of fusion entity. 


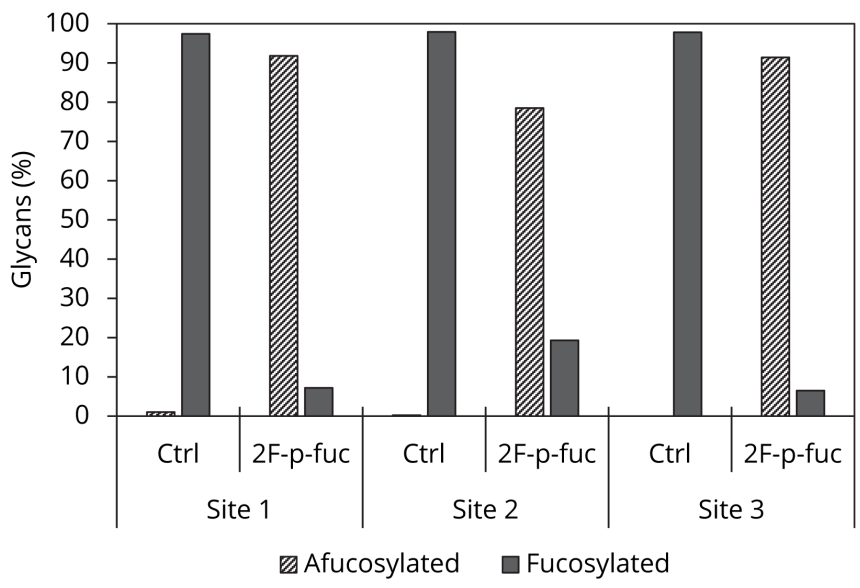

Figure 9.5 - Level of total afucosylated and fucosylated species at the three glycosylation sites of the antibody fusion molecule expressed by cell line B. Both afucosylated and fucosylated forms include galactosylated and sialylated forms. High mannose species were not considered.

tively. The same order was also applicable to the Fc $\gamma$ RIIIb affinity. The binding affinity constant of the control, raffinose, kifunensine and the fucose analog reached 9.6, 7.9, 2.5 and 1.4 $\mu \mathrm{M}$, respectively. The ADCC reporter potency results presented similar effects consistent with the Fc $\gamma$ RIIIa results and with the levels of afucosylated and high mannose gycans (figure 9.7D). Raffinose treated samples displayed a twofold increase, while kifunensine and 2F-p-fucose resulted in 8 -fold and 18-fold increases, respectively, of the relative ADCC reporter potency.

Among the supplements, $\mathrm{Mn}, \mathrm{Gal}$ and $\mathrm{NH}_{4}$, as well as the $\mathrm{mAb}$ treated with GalT, significant changes of the relative CDC potencies were observed, as well as for the relative C1q with GalT treated product (figures 9.7E and F). Mn \& Gal supplemented cultures entailed a relative Clq potency of $110 \%, \mathrm{NH}_{4}$ and GalT featured 105 and $115 \%$, respectively. Due to the inherent variability between the independent assays, only the increase of the GalT-treated samples was significant. The relative mAb CDC potency of the Mn \& Gal and ammonium supplemented cultures, as well as treatment with GalT altered the relative CDC potency, reaching 117, 90 and $128 \%$, respectively. Hence, the increasing levels of galactosylation correlated with higher relative CDC potency. None of the medium supplements nor the GalT treated samples effected significant impacts on the Fab affinity to the tumor necrosis factor- $\alpha$ (data not shown).

As expected, afucosylation (of cell line B) resulted in increased affinities for Fc $\gamma$ RIII V158 and Fc $\gamma$ RIII F158 (figures 9.8A and B). For the former, the $K_{D}$ of the control amounted to $0.9 \mu \mathrm{M}$, for raffinose, $1 \mu \mathrm{M}$ kifunensine (no results available for $30 \mu \mathrm{M}$ kifunensine) and $2 \mathrm{~F}$-p-fucose to 0.8, 0.5 and $0.1 \mu \mathrm{M}$, respectively. The Fc $\gamma$ RIII F158 dissociation affinity constant of the control reached 1.7 $\mu \mathrm{M}$. Raffinose, $1 \mu \mathrm{M}$ kifunensine and 2F-p-fucose supplementation resulted in values of 1.6, 1.0 and $0.2 \mu \mathrm{M}$, respectively. Neither cell culture medium supplementation nor enzymatic glycoengineering significantly altered the Fab activity including the cell-based ligand binding activity and Biacore receptor binding. The results of the two assays were 
A - Site 1

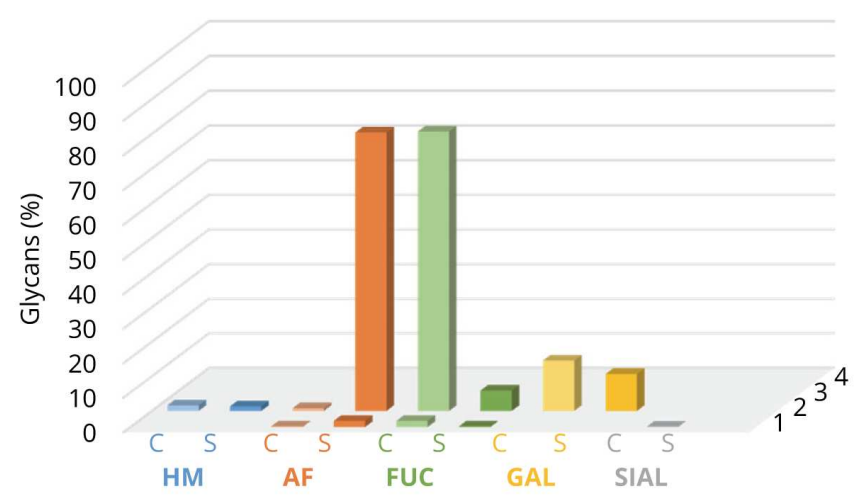

B - Site 2

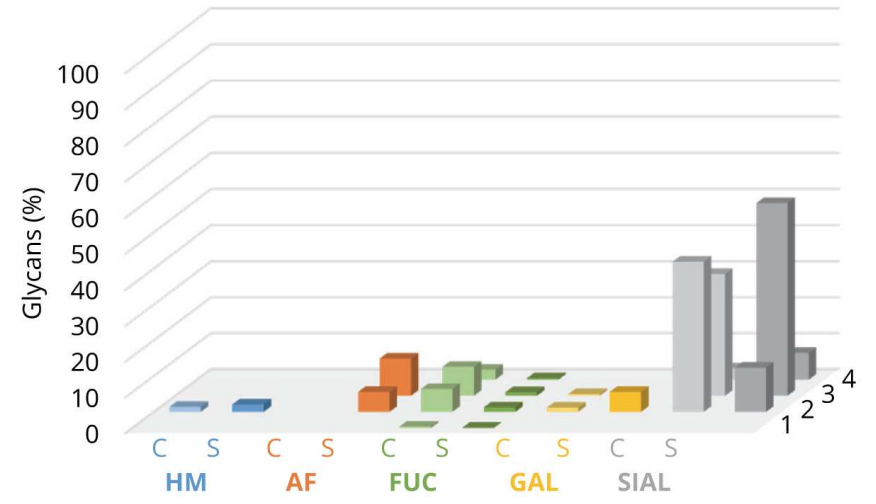

C - Site 3

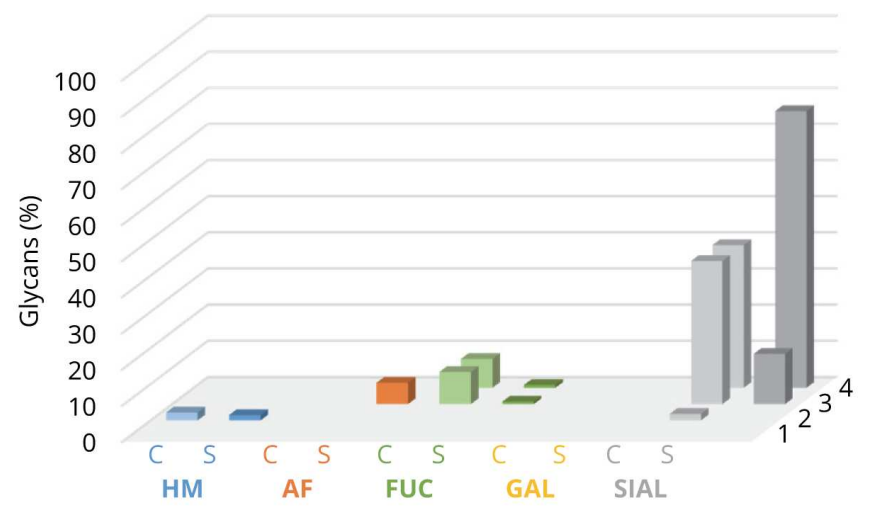

Figure 9.6 - Glycan antennarity of control and culture supplemented with 2F-p-fucose of cell line B. (A) Glycan site 1 located at Fc domain. (B) Glycan site 2 located at non-Fc-part of fusion entity. (C) Glycan site 3 located at non-Fc-part of fusion entity. The number of branches is shown on the right-hand side of the charts. 

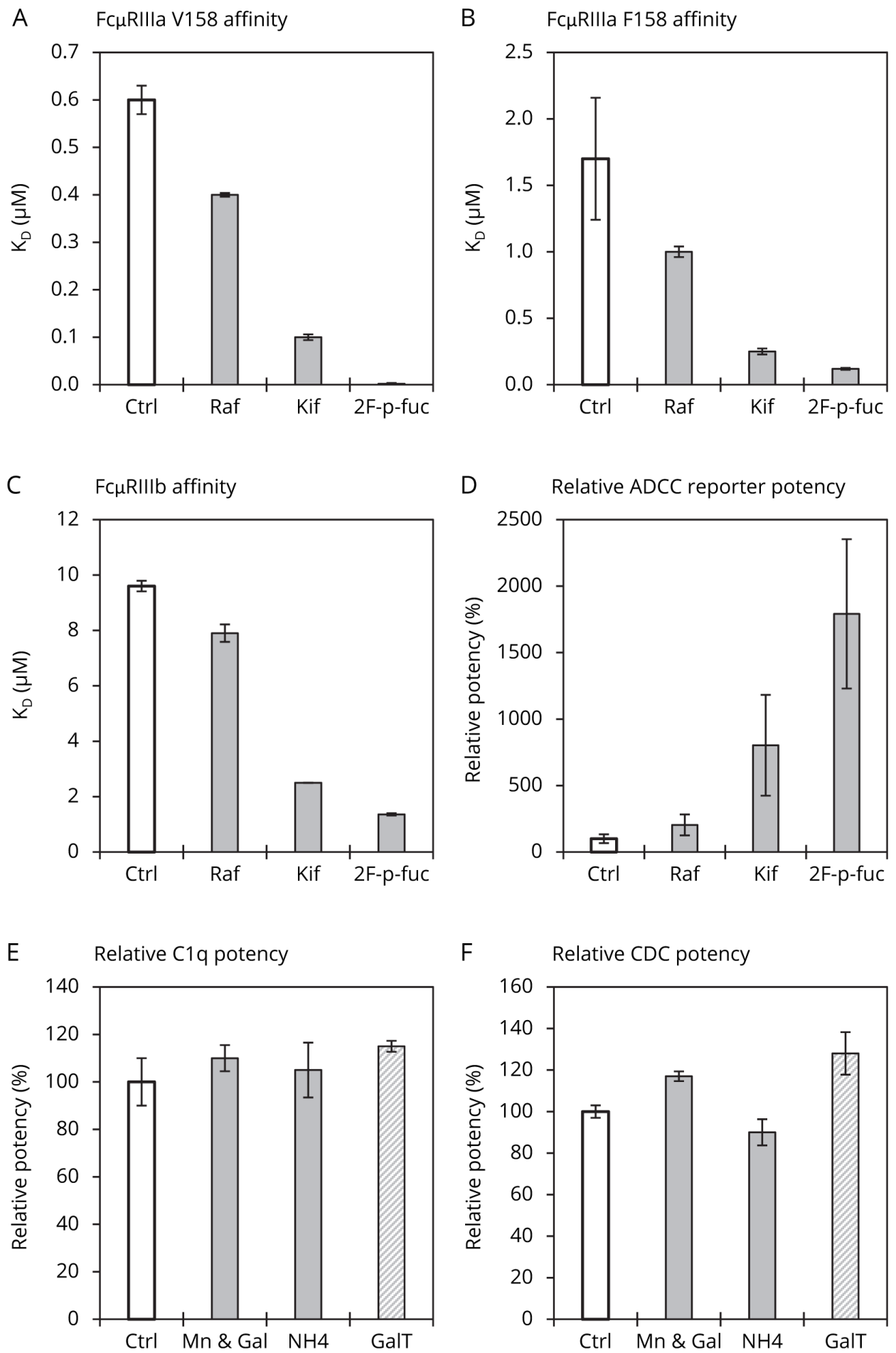

Figure 9.7 - (A) Fc $\gamma$ RIIIa F158 affinity of cell line A. (B) Fc $\gamma$ RIIIa V158 affinity. (C) Fc $\gamma$ RIIIb affinity. (D) Relative ADCC reporter potency. (E) Relative Clq potency. (F) Relative CDC potency. The results were released as averages of independent assays. The error bar mark the variability. 
comparable (data not shown). The glycovariants did not significantly affect the fusion entity binding affinity according to the cell-based reporter gene assay (figure 9.8C). While solely high mannose and afucosylated variants induced weak increases of the fusion entity activity in the cell-based assay, increased levels of high mannose, afucosylated, galactosylated, and sialylated glycans significantly enhanced the activity in the Biacore assay (figure 9.8D).
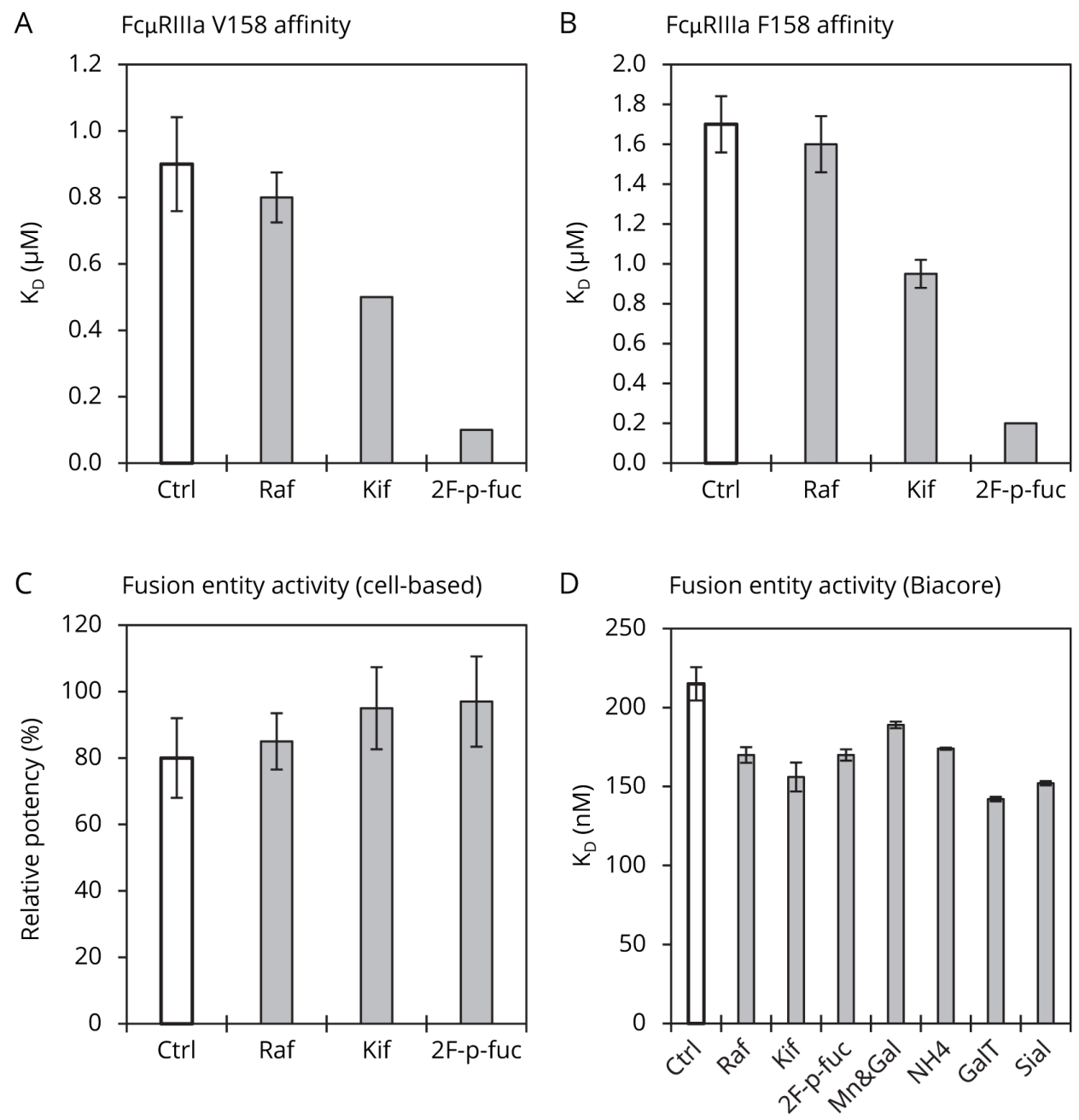

Figure 9.8 - (A) Fc $\gamma$ RIIIa F158 affinity. (B) Fc $\gamma$ RIIIa V158 affinity. (C) Fusion entity cell-based activity. (D) Fusion entity activity by Biacore. The results were released as averages of three independent assays. The error bar mark the variability.

\subsection{Discussion}

These results highlight that cell culture medium supplementation is a powerful tool to induce metabolic changes that affect the glycosylation pathway. A great diversity of a variety of glycans resulted while limiting detrimental effects on the cell line A culture performance. Although the 
high kifunensine concentration of the cell line B cultures entailed impaired growth, sufficient amounts of protein were expressed for the subsequent glycan and bioactivity analyses. This alkaloid proved to be the supplement of choice to generate a great abundance of high mannose glycans in both cell lines. While the identified amount of HM for cell line A amounted to $80.2 \%$, the real level of high mannose species was probably even higher. According to the reported mechanisms ${ }^{91}$, it is unlikely that high amounts of sialylated species would be generated. It is assumed that the reported $6 \%$ sialic acid were due to a $2 \mathrm{AB}$-UPLC peak miss-assignment. The peaks of the control culture were well characterized in the frame of the method set-up. Nevertheless, since the kifunensine supplementation brought about a variety of atypical peaks, the established routine peak assignment procedure presumably identified hybrid glycans as sialylated species. Mass spectroscopy technology may be used to identify the species accurately. Because the purpose of this study was the evaluation of the potential of media design for bioactivity assessment, the identification of those peaks was not further investigated. The hypothesis is further backed up by the results of the glycopeptide method that was used for the more complex cell line B molecule. The glycopeptide measurement detected an abundance of 99.7\% HM (10.2\% Man8 and 88.7\% Man9) at $30 \mu \mathrm{M}$. Interestingly, cell line B required a 30-fold higher concentration to effectively inhibit glycan maturation. Raffinose can be used effectively to generate predominantly Man5. It also favored galactosylation. Its effect on the glycosylation metabolism of cell line B was significantly weaker. Nonetheless, the productivity was strongly impaired. The metabolism of cell line B was apparently more sensitive to the important concentration of raffinose in the medium although the medium osmolality was maintained constant. The trisaccharide entailed a substantially lower productivity and thereby the harvest titer amounted to $680 \mathrm{mg} / \mathrm{L}$ (control: $1281 \mathrm{mg} / \mathrm{L}$ ). Specific inhibition of fucosylation resulted with 2F-p-fucose addition. The constant medium osmolality approach enabled high galactose concentrations that, in combination with $\mathrm{Mn}$, allowed to obtain a level of $50 \%$ of galactosylated species.

The environment of the glycosylation site plays a pivotal role in the glycan distribution. While site 1, located in the $\mathrm{CH} 2$ domain of the antibody moiety of the fusion molecule (cell line B), featured a similar glycan distribution to classical mAbs, sites 2 and 3 were characterized by a high degree of sialic acid. These result show that despite equal sialyltransferase activity and substrate levels in the Golgi apparatus, and as a consequence, at all three glycosylation sites of the molecule, terminal sialic acid was not favored at the $\mathrm{CH} 2$ domain. Steric hindrance presumably obstructed this process. The lower degree of branching further supports this hypothesis. The specific surroundings of site 2 and 3 also effected a distinct pattern of the sialylated species, in particular. In the non-supplemented cultures, site 2 had a slight preference for triantennary and site 3 for tetraantennary sialylated forms. In addition, site 3 featured a substantially greater number of different glycan species than site 2 . Astonishingly, 2F-p-fucose supplementation strongly enhanced the preference for tetraantennary sialylated forms. Mainly afucosylated, $73 \%$ of the sialylated forms at site 2 featured three branches, while site 3 contained $85 \%$ tetraantennary sialylated entities. The presence or absence of core fucosylation exhibited a central function in the fusion moiety glycan maturation. While in 
non-supplemented conditions fucosylated glycans with four branches with neither terminal galactose nor sialic acid (FA4) were present, no A4 glycans appeared at site 2 and site 3 in the 2F-p-fucose supplemented culture. Thus, the absence of the core fucose further favored terminal sialic acid, which may have effected greater accessibility of the glycosylation site or enhanced stability of the branched and matured glycan due to increased interaction with the amino acids of the polypeptide backbone nearby. Moreover, the specific environment of each site plays an important role in the nature and the strength of the hydrogen bonds and Van der Waals forces ${ }^{314}$. Manganese and galactose supplementation considerably increased the level of galactosylation at site 1 from 14.6 to $53.4 \%$. However, this strategy failed at site 2 as well as site 3. The two media additives effected a significant decrease of the fucosylated forms, but the level of galactosylation did not change. Instead, the sialylated forms increased by the same order of magnitude than the reduction of fucosylation. It seems that the increased availability of terminal galactose enhanced sialylation, and thus, the overall level of galactosylation remained unchanged. Ammonium was the only medium supplement that produced significant galactosylation increases at site 2 . This is surprising as it generally inhibits galactosylation due to the rise of the intra-Golgi $\mathrm{pH}^{237}$. The only way to generate mainly galactosylated glycans at the fusion-moiety sites was by means of enzymatic glycoengineering of the supernatant. Sialidase cleaved the terminal sialic acid as the significant decrease of that peak implies. As a result, the level of galactosylation increased to 82 and $79 \%$, respectively. GalT entailed $99 \%$ galactosylation at the $\mathrm{CH} 2$ domain, while at the others sites galactosylation slightly increased and sialic acid further increased. The underlying mechanism leading to higher levels of sialic acid are not fully understood and require further investigation. Due to sample or substrate contamination and insufficient supernatant purification, residual sialyltransferase and sialic acid may have been still present. On the other hand, to some extent, this can be linked to a redistribution of di-, tri-, and tetra-antennary species. The hypothesis that the subsequent Protein A purification may not retain newly created species and thus may have led to an altered glycan distribution has to be confirmed. Also, it shall be investigated if small portions of the host cell proteins ensemble contained sialyltransferase, which possibly transferred sialic acid from host cell proteins to the recombinant protein. The performance of GalT used for cell line A and the one for 2 differed. GalT treatment of the former generated a higher ratio of mono-galactosylated than di-galactosylated forms $(8: 1)$, while the second predominantly formed di-galactosylated species at the $\mathrm{CH} 2$ domain (1.0: 1.4). As a parallel approach, enzymatic glycoengineering is a valuable tool to further extend the glycosylation modulator library. Nonetheless, the choice of the right enzyme is pivotal. The two GalT enzymes sourced from two different suppliers exhibited different effects.

Both cell culture medium supplementation and enzymatic glycoengineering produced substantial effects on receptor affinities and potencies. For cell line A, 2F-p-fucose, and to a slightly lesser extent, kifunensine increased the Fc $\gamma$ RIII binding affinities. The afucosylated mAbs exhibited 39-fold enhanced binding with Fc $\gamma$ RIIIa V158, 14-fold with Fc $\gamma$ RIIIa F158 and 7-fold with Fc $\gamma$ RIIIb. The high mannose variants generated with raffinose featured intermediate affinity increases. The relative ADCC reporter potencies of these three supplements were 
aligned with trends observed for the Fc $\gamma$ RIII binding affinities. The level of galactosylation influenced both C1q and CDC potencies. Even though Mn \& Gal supplementation resulted in $50 \%$ galactosylation, the variants were characterized by lower C1q and CDC potencies than the GalT samples. Different ratios between FA2G1 3-arm and FA2G1 6-arm as well as between FA2G1 and FA2G2 may have effected this behavior. Cell line B featured a similar pattern to cell line A for Fc $\gamma$ RIII receptor binding. It was most strongly affected by the degree of fucosylation. The afucosylated variants yielded 9-fold stronger Fc $\gamma$ RIII V158 and F158 affinities. Slightly higher levels of high mannose glycans due to the presence of $1 \mu \mathrm{M}$ kifunensine already effected significant binding affinity enhancements, highlighting the importance of the level of high mannose glycans in cell line B. Interestingly, in both molecules the glycosylation did not impact the Fab binding activities. Moreover, the glycosylation had weak effects on the binding activity of the fusion entity of cell line $\mathrm{B}$. The results suggest that the glycosylation of the recombinant protein most strongly impacts its Fc activity, and given the similar trends of both molecules, it is assumed that the Fc-glycosylation pattern has the greatest effect on the affinity of the fusion antibody. The data highlight that cell culture medium supplementation and enzymatic glycoengineering offer a valuable and straightforward approach to generate great glycan diversity, which induces significant responses in the corresponding bioactivity assays.

\subsection{Conclusion}

Cell culture medium supplementation with a variety of compounds interfering with the direct and indirect glycosylation pathways was successfully applied to generate extreme glycan variants. Important levels of high mannose and afucosylated glycans of two different molecules were generated. While, this approach enabled a level of galactosylation of $50 \%$, high degrees were achieved, using enzymatic glycoengineering. Cell-culture supplementation proved to be ineffective for significant increases of galactosylation of the highly sialylated antibody fusion molecule. Solely sialidase treatment generated highly galactosylated variants. Glycopeptide analysis also showed that the environment, namely the steric effects, shape the glycan pattern at each specific site. Sialylation was low at the Fc-glycan sites of both molecules, while it was the predominant glycan group including a great variety of highly branched entities at the two fusion-entity sites. Furthermore, the afucosylated glycans promoted terminal sialic acid and as a consequence, the protein expressed in medium supplemented with $2 \mathrm{~F}$-p-fucose featured substantially higher levels of sialylation. Afucosylated and high mannose variants of both $\mathrm{mAb}$ and antibody fusion molecule substantially increased Fc $\gamma$ III receptor binding affinities and ADCC reporter activities. Clq and CDC potencies increased with higher level of $\mathrm{mAb}$ galactosylation. In conclusion, cell culture medium supplementation has a great potential to induce substantial glycan distribution changes of both classical mAb and complex molecules having several glycosylation sites, and thus to assess their effects on the biological activity. 



\section{Chapter 10}

\section{Concluding Remarks and Perspectives}

Recombinant protein quality modulation has become an inherent part of the cell culture process development workflow for new biological entities and biosimilars. During several decades, the biotechnology industry was mainly concentrating its effort to improve productivity, which has certainly paid off. Nonetheless, the advent of biosimilars triggered the innovation of a variety of technologies to alter the quality attributes of biotherapeutics. The present work consisted in levering the central role of cell culture medium to induce changes in the metabolic pathways that are directly and indirectly involved in post-translational modifications. Rather than calling on cell-line-engineering techniques and cell-culture-process optimization, the focus was directed towards medium and feed supplementation to alter, in particular, glycosylation and to initiate the study of inherent low-molecular-species formation of the in-house platform process, working with both common cell culture media components and new compounds. First of all, the glycosylation modulation library was extended, using high throughput 96-deepwell plate and shake tubes. Chapter 4 describes how raffinose supplementation reproducibly increased the levels of high mannose glycans in two different cell lines. The findings were confirmed in controlled $\mathrm{pH}-, \mathrm{O}_{2}$ - and $\mathrm{CO}_{2}$-conditions in 3.5-L bioreactors. The presence of raffinose influenced the cell metabolism, thus affecting cell culture performance. The supplement also induced changes in intracellular nucleotide levels and gene regulation. In chapter 5 , the addition of fluorinated galactose analogs in media and feed solutions specifically reduced galactosylation. Furthermore, spermine and L-ornithine inhibited the attachment of terminal galactose on the GlcNAc moiety. Then in chapter 6 , in an attempt to expand the scope of quality modulation, various disulfide-bridge-reducing agents were supplemented in cell culture experiments. They showed that reduction is probably at the core of the observed level of fragmentation in the proprietary cell-culture-platform process. Rather than performing single, univariate screening in high throughput fed-batch cultures, the effect of seventeen medium supplements on product quality were successfully assessed in five parallel 96-deepwell plate experiments as described in chapter 7. The compounds effected wide glycosylation pattern ranges. The best modulating substances to improve the glycosylation profile with respect to the specifications for biosimilarity were identified for 
the subsequent evaluation in shake tubes by a three-step multivariate analysis approach calling on principal component analysis, evaluation of modulation performance and selection following a hierarchical order by means of a decision tree. Combining parallel testing and the use of multivariate tools proved to be particularly effective. The glycosylation profile of the shake tube experiments was substantially enhanced. Moreover, non-targeted metabolomic intra- and extracellular profiling provided many insights on the time course of a plethora of metabolites (chapter 8). The four mAb 3.5-L bioreactor processes exhibited distinct profiles. First of all, univariate analysis was carried out to identify a number of metabolites featuring similar profiles to the extracellular lactate profiles. The potential of modelling the glycosylation profile was shown based on the extracellular metabolite data. The partial-least-square (PLS) projection on latent variables model calibrated with three of the four processes predicted the glycan pattern of the fourth process well. Multivariate modelling is sought after, in particular in large-scale routine manufacturing, to tightly control the process within the defined operating range, using real-time data. Finally, as shown in chapter 9, further extension of the glycan modulating compound library enabled the generation of a variety of extreme glycan variants of the major glycan species including high mannose, afucosylated, low- and highly galactosylated, and low- and highly sialylated forms of both a classical mAb and an antibody fusion molecule with three distinct $\mathrm{N}$-glycosylation sites. In that frame, enzymatic glycoengineering of galactosylation and of sialic acid of the purified protein was evaluated. The generated glycoforms induced significant responses in binding and activity assays. Hence, medium and feed supplementation lends itself to assess the effect of the induced glycosylation changes on the biological activity, and in addition, the impact of the remaining differences between the biosimilar in development and the reference medicinal product (RMP).

In this thesis, exclusively $\mathrm{CHO}-\mathrm{S}$ and $\mathrm{CHO}-\mathrm{K} 1$ derived cell lines were used. It would be desirable to extent this work to other rodent cell lines, such as SP2/0 and NS0, as well as to human cell lines. For future development projects, the knowledge for other rodent cell lines would be of great value. Further understanding of the glycosylation modulation techniques, in particular in human cell lines, would potentially benefit other research areas in diagnostics and potentially in the field of oncology. It is suggested to extend the design-of-experiment approach for enhanced mechanistic learnings that would be transferable to other cell lines and processes. Along this endeavor, the introduction of additional cell culture process parameters and metabolite data will lay a foundation for the creation of predictive process models as early as possible in the process development workflow.

The generated data regarding the formation of low-molecular-weight species indicate that disulfide brige reduction is one of the underlying mechanisms driving the disassembly of the expressed recombinant proteins in the proprietary platform process. Further work is required, namely more specific tests enabling to confirm the described hypotheses. There are also a number of compounds that potentially inhibit the thioredoxin reductases. The final goal would be to redesign the proprietary medium to limit LMW species substantially. The modulation of the charge variants was out-of-scope in the present work. Additional studies aiming to understand how the various post-translational modifications are involved in the changes of 
the charge profile will be of great value for future process development programs.

It is suggested to combine media design and the optimization of process parameters in controlled cell culture equipment. Micro-scale bioreactors would definitely be the tool of choice. In chapter 7, a temperature decrease was also included in shake tube experiments. By doing so, the overall quality-modulation response was increased. Last but not least, routine manufacturing facilities would benefit from further modelling efforts resulting in multi-parameter models that include kinetics and potentially transcriptomics data. The development of reliable and easy-to-use models in combination with on-line measurement technologies, such as optical probes and Raman spectroscopy, are strongly encouraged. Nonetheless, sophisticated methodologies will be required to cope with the complex mathematical data treatment.

In conclusion, the results of this work demonstrate the great potential of media design to fine-tune the quality profile of recombinant proteins within the potential of the selected cell line. The capacity of quality modulation compound library largely responds to the fine-tuning requirements in the development of new biotherapeutics. The high-throughput experimental strategy and the multivariate modelling provide rational, systematic and more rapid ways to identify well performing modulating compounds and to lever mechanistic understanding for glycosylation control in routine manufacturing. The leverage of the key learnings of this present work provided an approach to assess the impact of the induced glycan changes on the biological activity. It further shows the sensational features of media supplementation that allows to generate a great diversity of the glycan pattern. 

Appendix A

\section{Experimental Designs for Efficient Screening of Cell Culture Media Supplements to Improve the Product Quality (Chapter 7)}

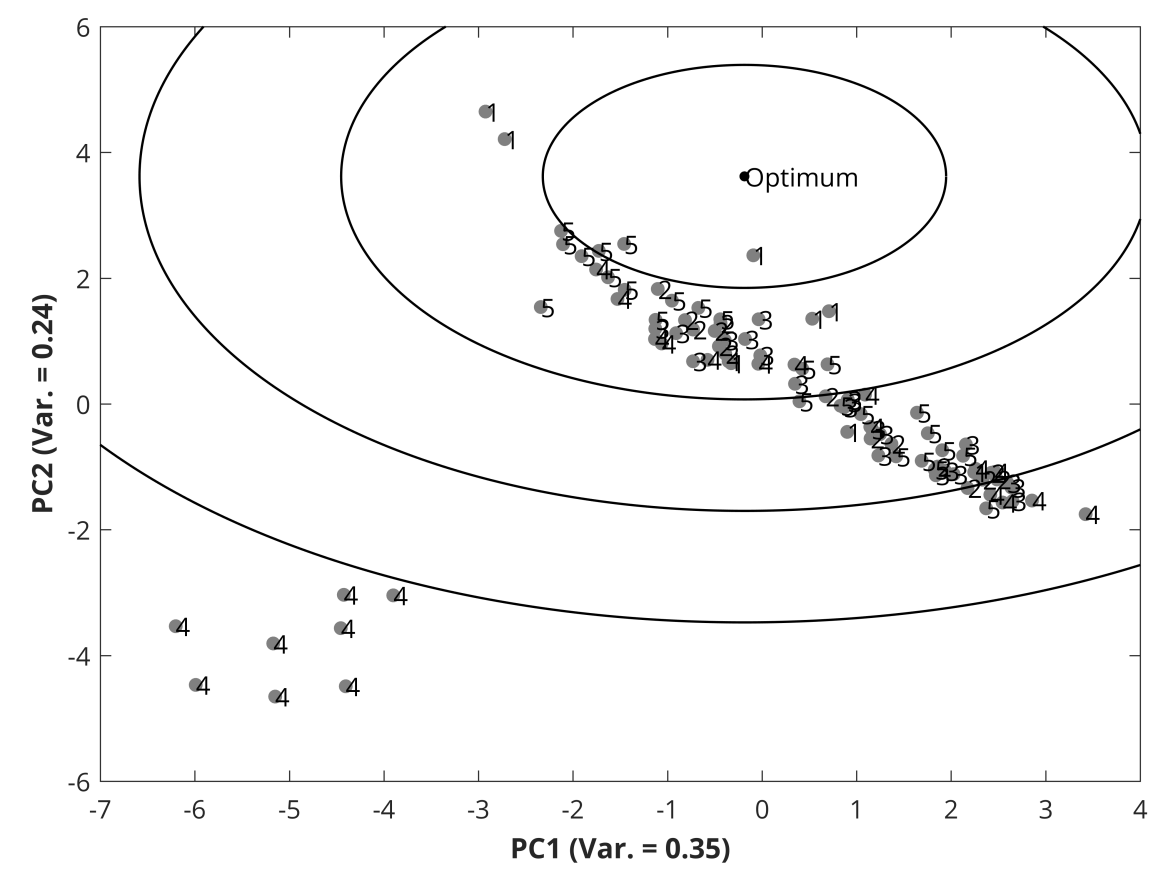

Figure A.1 - PCA score plot of 96-DWP experiments. The PC1 explains $35 \%$ of variance, and PC2 24\%. The different experiments are marked with their DoE group. 


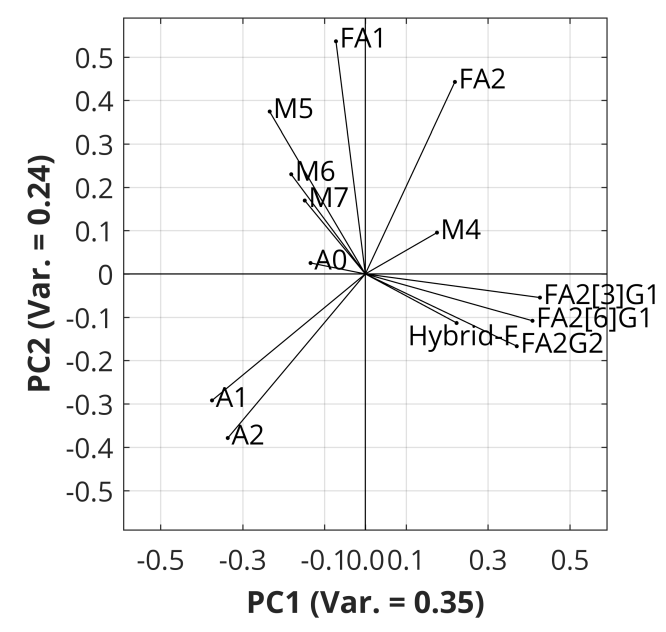

Figure A.2 - PCA loading plot of 96-DWP experiments.

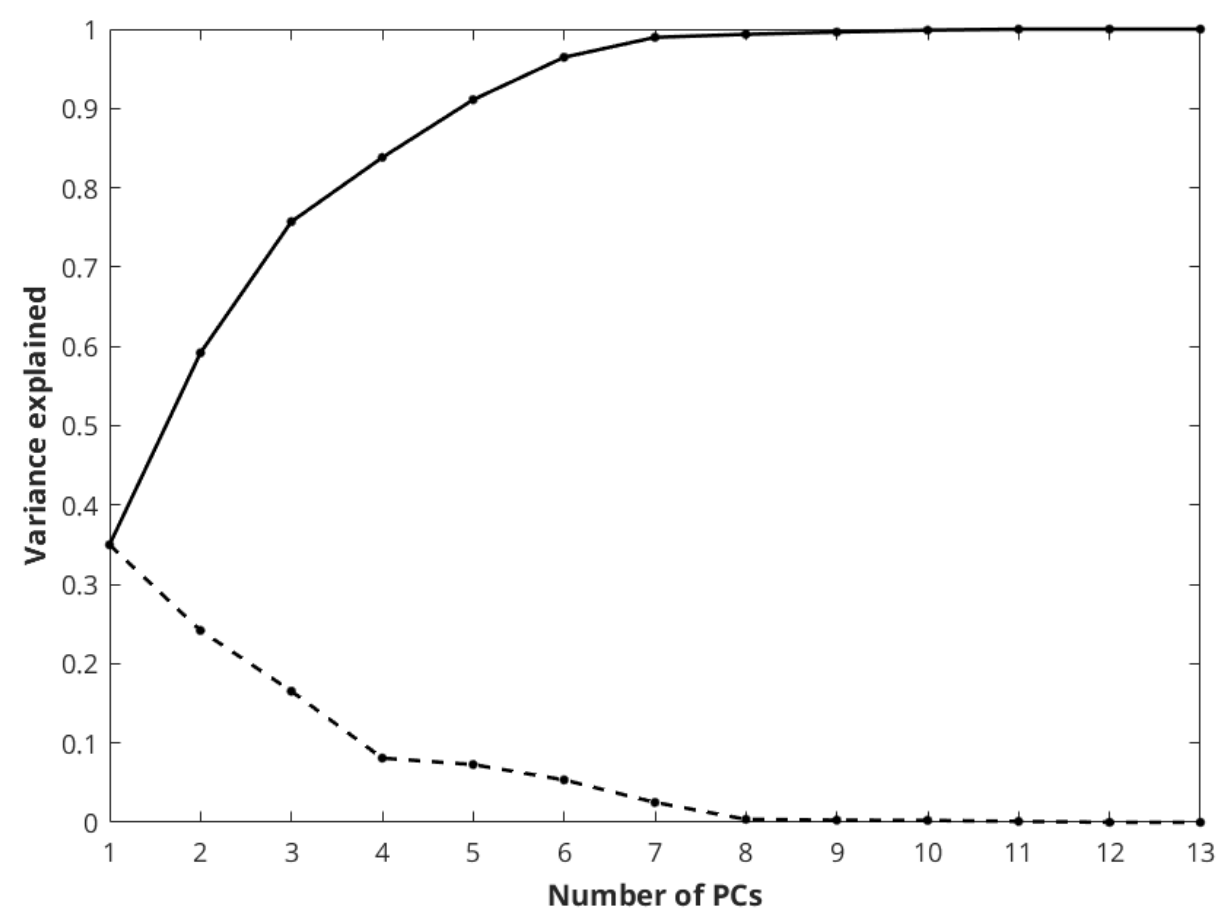

Figure A.3 - PCA on 96-DWP experiments: Cumulative variance explained by the PCs (solid line) and Scree plot showing variance explained by each PC (dashed line). The characteristic elbow at $\mathrm{PC}=4$ indicates that the relevant information is likely to be captured by the first 3 PCs. 
Table A.1 - Experimental design of group 1 in 96 DWP.

\begin{tabular}{cccccc}
\hline Well & Factor 1 & Factor 2 & Factor 3 & Mn & Asn \\
\hline 1 & 0 & 0 & 0 & 0 & 0 \\
2 & 0 & 0 & 0 & 0 & 0 \\
3 & -1 & -1 & -1 & -1 & -1 \\
4 & -1 & -1 & -1 & -1 & -1 \\
5 & -1 & -1 & -1 & 0 & 1 \\
6 & 1 & 1 & 1 & 1 & -1 \\
7 & -1 & 1 & 1 & 1 & 1 \\
8 & 1 & -1 & -1 & 1 & -1 \\
9 & 1 & 1 & -1 & -1 & -1 \\
10 & -1 & -1 & 1 & 1 & -1 \\
11 & -1 & 1 & -1 & -1 & 1 \\
12 & 1 & 1 & -1 & 1 & 1 \\
13 & -1 & 1 & 1 & -1 & -1 \\
14 & -1 & -1 & -1 & 1 & 1 \\
15 & 1 & -1 & 1 & 1 & 1 \\
16 & -1 & 1 & -1 & 1 & -1 \\
17 & -1 & -1 & 1 & -1 & 1 \\
18 & 1 & 1 & 1 & -1 & 1 \\
19 & 1 & -1 & -1 & -1 & 1 \\
20 & 1 & -1 & 1 & -1 & -1 \\
21 & 1 & -1 & 1 & -1 & -1 \\
22 & 1 & 0 & 0 & 1 & 1 \\
23 & 0 & 0 & -1 & -1 & 1 \\
24 & 1 & 0 & -1 & 0 & -1 \\
25 & 1 & -1 & 1 & 0 & 0 \\
26 & 0 & -1 & 0 & 1 & -1 \\
27 & -1 & 1 & 0 & 0 & 1 \\
28 & -1 & 0 & 1 & 1 & 0 \\
29 & 0 & -1 & 1 & 1 & 1 \\
30 & 1 & 1 & 0 & -1 & 0 \\
31 & -1 & 1 & 1 & -1 & 0 \\
32 & 0 & 1 & -1 & 1 & 0 \\
33 & 0 & 1 & 1 & 0 & -1 \\
\hline & & & & & \\
& 1 & 1 & 1
\end{tabular}


Table A.2 - Experimental design of group 2 in 96 DWP.

\begin{tabular}{cccccc}
\hline Well & Factor 1 & Factor 2 & Factor 3 & Mn & Asn \\
\hline 1 & 0 & 0 & 0 & 0 & 0 \\
2 & 0 & 0 & 0 & 0 & 0 \\
3 & -1 & -1 & -1 & -1 & -1 \\
4 & -1 & -1 & -1 & -1 & -1 \\
5 & -1 & -1 & -1 & 0 & 0 \\
6 & 1 & -1 & -1 & -1 & 1 \\
7 & -1 & 1 & 1 & 1 & 1 \\
8 & 1 & -1 & 1 & 1 & 1 \\
9 & 1 & 1 & -1 & -1 & -1 \\
10 & 1 & -1 & -1 & 1 & -1 \\
11 & 1 & 1 & -1 & -1 & -1 \\
12 & 1 & 1 & 1 & -1 & 1 \\
13 & 1 & 1 & 1 & 1 & -1 \\
14 & -1 & -1 & -1 & 1 & 1 \\
15 & -1 & -1 & 1 & -1 & 1 \\
16 & 1 & -1 & 1 & -1 & -1 \\
17 & -1 & -1 & 1 & 1 & -1 \\
18 & -1 & 1 & -1 & 1 & -1 \\
19 & 1 & 1 & -1 & 1 & 1 \\
20 & -1 & 1 & 1 & -1 & -1 \\
21 & -1 & 1 & -1 & -1 & 1 \\
22 & 1 & 0 & 1 & 0 & -1 \\
23 & -1 & -1 & 0 & -1 & 1 \\
24 & 1 & -1 & 1 & 1 & 0 \\
25 & -1 & 0 & 0 & 1 & -1 \\
26 & 1 & 1 & 0 & 0 & 1 \\
27 & -1 & 1 & 1 & 0 & -1 \\
28 & 0 & 1 & 0 & -1 & -1 \\
29 & 1 & -1 & 0 & -1 & 0 \\
30 & 0 & 0 & -1 & -1 & 1 \\
31 & 0 & 1 & 1 & 1 & 0 \\
32 & -1 & 0 & 1 & -1 & 0 \\
33 & 0 & -1 & 1 & 0 & 1 \\
\hline & & & & &
\end{tabular}


Table A.3 - Experimental design of group 3 in 96 DWP.

\begin{tabular}{cccccc}
\hline Well & Factor 1 & Factor 2 & Factor 3 & Mn & Asn \\
\hline 1 & 0 & 0 & 0 & 0 & 0 \\
2 & 0 & 0 & 0 & 0 & 0 \\
3 & -1 & -1 & -1 & -1 & 0 \\
4 & -1 & -1 & 1 & 1 & -1 \\
5 & -1 & -1 & 1 & -1 & -1 \\
6 & 1 & 1 & -1 & 1 & -1 \\
7 & -1 & -1 & -1 & 1 & -1 \\
8 & 1 & -1 & 1 & 1 & -1 \\
9 & 1 & -1 & -1 & -1 & -1 \\
10 & 1 & 1 & 1 & 1 & 1 \\
11 & 1 & 1 & -1 & -1 & 1 \\
12 & -1 & 1 & -1 & 1 & 1 \\
13 & 1 & -1 & -1 & 1 & 1 \\
14 & 1 & 1 & 1 & -1 & -1 \\
15 & 1 & -1 & 1 & -1 & 1 \\
16 & -1 & 1 & 1 & 1 & -1 \\
17 & -1 & -1 & 1 & 1 & 1 \\
18 & -1 & 1 & -1 & -1 & -1 \\
19 & -1 & -1 & 0 & 0 & 1 \\
20 & 0 & -1 & -1 & -1 & 1 \\
21 & 0 & 0 & -1 & 1 & -1 \\
22 & -1 & 0 & 1 & 1 & 1 \\
23 & 1 & 1 & -1 & 0 & 0 \\
24 & -1 & 0 & 1 & -1 & 0 \\
25 & 1 & 0 & 0 & -1 & 1 \\
26 & 1 & 0 & 1 & 0 & -1 \\
27 & 0 & 1 & 1 & 0 & 1 \\
28 & -1 & 1 & -1 & 0 & -1 \\
\hline & & & & &
\end{tabular}


Table A.4 - Experimental design of group 4 in 96 DWP.

\begin{tabular}{cccccc}
\hline Well & Factor 1 & Factor 2 & Factor 3 & Mn & Asn \\
\hline 1 & -1 & -1 & -1 & 0 & 0 \\
2 & -1 & -1 & -1 & -1 & -1 \\
3 & -1 & -1 & -1 & -1 & -1 \\
4 & -1 & -1 & -1 & 0 & -1 \\
5 & 0 & 0 & 0 & 1 & 1 \\
6 & 0 & 0 & 0 & -1 & 1 \\
7 & -1 & -1 & -1 & 1 & 1 \\
8 & -1 & 1 & -1 & 1 & -1 \\
9 & 1 & 1 & -1 & -1 & -1 \\
10 & 1 & 1 & 1 & 1 & 1 \\
11 & -1 & -1 & -1 & 1 & -1 \\
12 & 1 & 1 & 1 & -1 & 1 \\
13 & -1 & 1 & 1 & 1 & -1 \\
14 & 1 & -1 & -1 & 1 & 1 \\
15 & 1 & -1 & -1 & 1 & -1 \\
16 & -1 & -1 & -1 & -1 & 1 \\
17 & -1 & 1 & 1 & -1 & -1 \\
18 & 1 & 1 & -1 & -1 & -1 \\
19 & -1 & -1 & 1 & -1 & 1 \\
20 & 1 & -1 & 1 & 1 & -1 \\
21 & 1 & -1 & 1 & 1 & -1 \\
22 & -1 & 1 & -1 & 1 & 1 \\
23 & -1 & -1 & 1 & 0 & 1 \\
24 & 1 & 0 & 0 & 1 & 0 \\
25 & 0 & -1 & 0 & -1 & -1 \\
26 & 0 & 0 & 1 & 1 & 0 \\
27 & -1 & 0 & 0 & -1 & 0 \\
28 & 1 & 0 & -1 & 0 & -1 \\
29 & -1 & 0 & -1 & 1 & 1 \\
30 & 0 & 1 & -1 & 1 & -1 \\
31 & 1 & 1 & 0 & 0 & 0 \\
32 & 1 & -1 & -1 & 0 & 0 \\
33 & -1 & 1 & 0 & -1 & -1 \\
34 & 0 & 1 & -1 & -1 & -1 \\
35 & 1 & 0 & -1 & 1 & 0 \\
\hline & & & & &
\end{tabular}


Table A.5 - Experimental design of group 5 in 96 DWP.

\begin{tabular}{cccccc}
\hline Well & Factor 1 & Factor 2 & Factor 3 & Mn & Asn \\
\hline 1 & -1 & -1 & -1 & 0 & 0 \\
2 & -1 & -1 & -1 & -1 & -1 \\
3 & -1 & -1 & -1 & -1 & 1 \\
4 & 0 & 0 & 0 & -1 & -1 \\
5 & 0 & 0 & 0 & -1 & -1 \\
6 & -1 & -1 & -1 & 1 & -1 \\
7 & -1 & -1 & -1 & -1 & -1 \\
8 & -1 & -1 & -1 & -1 & 1 \\
9 & 1 & -1 & 1 & -1 & 1 \\
10 & 1 & 1 & -1 & 1 & -1 \\
11 & 1 & 1 & 1 & 1 & -1 \\
12 & -1 & 1 & 1 & 1 & 1 \\
13 & 1 & -1 & -1 & 1 & 1 \\
14 & -1 & -1 & 1 & 1 & 1 \\
15 & -1 & -1 & 1 & -1 & 1 \\
16 & 1 & -1 & -1 & -1 & -1 \\
17 & -1 & 1 & 1 & -1 & 1 \\
18 & 1 & 1 & -1 & 1 & -1 \\
19 & 1 & -1 & 1 & 1 & 1 \\
20 & 1 & 1 & 1 & 0 & 1 \\
21 & 1 & -1 & 1 & 1 & -1 \\
22 & -1 & 1 & -1 & 1 & 1 \\
23 & -1 & 1 & -1 & 0 & -1 \\
24 & -1 & -1 & -1 & 1 & -1 \\
25 & 1 & 0 & 1 & -1 & 1 \\
26 & 0 & 0 & 1 & -1 & 1 \\
27 & -1 & 0 & 0 & 1 & 0 \\
28 & 1 & 1 & 1 & 0 & 1 \\
29 & 0 & 1 & -1 & 0 & -1 \\
30 & 0 & 1 & 0 & -1 & 0 \\
31 & 1 & 0 & 0 & -1 & 0 \\
\hline & & & & &
\end{tabular}


Table A.6 - Design of experiments in TubeSpin bioreactor tubes.

\begin{tabular}{lccccc}
\hline & ST & Raffinose & Galactose & Enhancer 2 & T-Shift \\
\hline Center points for & 1 & 0 & 0 & 0 & -1 \\
evaluation of & 2 & 0 & 0 & 0 & -1 \\
repeatability & 3 & 0 & 0 & 0 & -1 \\
\hline D-optimal quadratic & 4 & -1 & 1 & 1 & -1 \\
design for 3 group & 5 & 1 & 1 & -1 & -1 \\
winners at 3 levels & 6 & 1 & -1 & 1 & -1 \\
& 7 & -1 & -1 & 1 & -1 \\
& 8 & -1 & -1 & 0 & -1 \\
& 9 & 0 & -1 & -1 & -1 \\
& 10 & 1 & 1 & 1 & -1 \\
& 11 & -1 & 1 & 0 & -1 \\
& 12 & -1 & 0 & 1 & -1 \\
& 13 & 1 & -1 & -1 & -1 \\
& 14 & -1 & 0 & -1 & -1 \\
& 15 & 0 & 1 & -1 & -1 \\
\hline Check of T-Shift at & 16 & 0 & 0 & 0 & 1 \\
center point & 17 & 0 & 0 & 0 & 1 \\
\hline D-optimal augmentation & 18 & 1 & 1 & -1 & 1 \\
of all experiments to & 19 & 1 & 1 & 1 & 1 \\
T-shift testing at 2 levels & 20 & -1 & -1 & -1 & 1 \\
& 21 & -1 & 1 & 1 & 1 \\
& 22 & 1 & -1 & 1 & 1 \\
\hline
\end{tabular}




\section{Bibliography}

[1] J. Zhu, Mammalian cell protein expression for biopharmaceutical production, Biotechnology Advances 30 (5) (2012) 1158-1170.

[2] W. S. Ahn, M. R. Antoniewicz, Towards dynamic metabolic flux analysis in CHO cell cultures, Biotechnology Journal 7 (1) (2012) 61-74.

[3] H. Schellekens, N. Casadevall, Immunogenicity of recombinant human proteins: causes and consequences, Journal of Neurology 251 Suppl (2004) II4-9.

[4] M. Aitken, Delivering on the Potential of Biosimilar Medicines: The Role of Functioning Competitive Markets Introduction, IMS Health March (2016) 1-37.

[5] C. Morrison, Fresh from the biotech pipeline—2015, Nature Biotechnology 34 (2) (2016) 129-132.

[6] A. Kantardjieff, W. Zhou, Mammalian cell cultures for biologics manufacturing, Vol. 139, Springer, 2014.

[7] M. Butler, A. Meneses-Acosta, Recent advances in technology supporting biopharmaceutical production from mammalian cells, Applied Microbiology and Biotechnology 96 (4) (2012) 885-894.

[8] F. M. Wurm, Production of recombinant protein therapeutics in cultivated mammalian cells, Nature Biotechnology 22 (11) (2004) 1393-1398.

[9] M. De Jesus, F. M. Wurm, Manufacturing recombinant proteins in kg-ton quantities using animal cells in bioreactors, European Journal of Pharmaceutics and Biopharmaceutics 78 (2) (2011) 184-188.

[10] P. K. Chugh, V. Roy, Biosimilars: Current scientific and regulatory considerations, Current Clinical Pharmacology 9 (1) (2014) 53-63.

[11] D. Bumbaca, C. A. Boswell, P. J. Fielder, L. A. Khawli, Physiochemical and Biochemical Factors Influencing the Pharmacokinetics of Antibody Therapeutics, The AAPS Journal 14 (3) (2012) 554-558. 
[12] N. Jenkins, L. Murphy, R. Tyther, Post-translational modifications of recombinant proteins: significance for biopharmaceuticals, Molecular Biotechnology 39 (2) (2008) 113118.

[13] H. Schellekens, Factors influencing the immunogenicity of therapeutic proteins, Nephrology, Dialysis, Transplantation 20 Suppl 6 (2005) vi3-vi9.

[14] P. Hossler, Protein glycosylation control in Mammalian cell culture: past precedents and contemporary prospects, Advances in Biochemical Engineering/Biotechnology 127 (2012) 187-219.

[15] M. H. Tao, S. L. Morrison, Studies of aglycosylated chimeric mouse-human IgG. Role of carbohydrate in the structure and effector functions mediated by the human IgG constant region, J Immunol 143 (8) (1989) 2595-2601.

[16] Q. Wang, M. Stuczynski, Y. Gao, M. J. Betenbaugh, Strategies for Engineering Protein N-Glycosylation Pathways in Mammalian Cells, in: A. Castilho (Ed.), Glyco-Engineering, Vol. 1321 of Methods in Molecular Biology, Springer New York, New York, NY, 2015, Ch. 20, pp. 287-305.

[17] M. Ivarsson, T. K. Villiger, M. Morbidelli, M. Soos, Evaluating the impact of cell culture process parameters on monoclonal antibody N-glycosylation, Journal of Biotechnology 188C (2014) 88-96.

[18] P. Hossler, S. F. Khattak, Z. J. Li, Optimal and consistent protein glycosylation in mammalian cell culture, Glycobiology 19 (9) (2009) 936-949.

[19] M. Jordan, M. Stettler, H. Broly, Will we ever find a perfect medium for mammalian cell culture?, Pharmaceutical Bioprocessing 1 (5) (2013) 411-413.

[20] S. S. Ozturk, M. R. Riley, B. O. Palsson, Effects of ammonia and lactate on hybridoma growth, metabolism, and antibody production, Biotechnology and Bioengineering 39 (4) (1992) 418-431.

[21] M. G. Vander Heiden, D. R. Plas, J. C. Rathmell, C. J. Fox, M. H. Harris, C. B. Thompson, Growth factors can influence cell growth and survival through effects on glucose metabolism, Molecular and cellular biology 21 (17) (2001) 5899-5912.

[22] M. Butler, M. Spearman, The choice of mammalian cell host and possibilities for glycosylation engineering, Current Opinion in Biotechnology 30C (2014) 107-112.

[23] C. K. Crowell, G. E. Grampp, G. N. Rogers, J. Miller, R. I. Scheinman, Amino acid and manganese supplementation modulates the glycosylation state of erythropoietin in a CHO culture system, Biotechnology and Bioengineering 96 (3) (2007) 538-549.

[24] S. Sha, C. Agarabi, K. Brorson, D.-Y. Lee, S. Yoon, N-Glycosylation Design and Control of Therapeutic Monoclonal Antibodies, Trends in Biotechnology 34 (10) (2016) 835-846. 
[25] Y. Rouiller, A. Périlleux, M.-N. Vesin, M. Stettler, M. Jordan, H. Broly, Modulation of $\mathrm{mAb}$ quality attributes using microliter scale fed-batch cultures, Biotechnology Progress 30 (3) (2014) 571-583.

[26] M. Jordan, D. Voisard, A. Berthoud, L. Tercier, B. Kleuser, G. Baer, H. Broly, Cell culture medium improvement by rigorous shuffling of components using media blending, Cytotechnology 65 (1) (2013) 31-40.

[27] Y. Rouiller, A. Périlleux, N. Collet, M. Jordan, M. Stettler, H. Broly, A high-throughput media design approach for high performance mammalian fed-batch cultures, mAbs 5 (3) (2013) 501-511.

[28] Y. Rouiller, J.-M. Bielser, D. Brühlmann, M. Jordan, H. Broly, M. Stettler, Screening and assessment of performance and molecule quality attributes of industrial cell lines across different fed-batch systems, Biotechnology Progress 32 (1) (2016) 160-170.

[29] K. P. Jayapal, K. F. Wlaschin, W.-S. H. Hu, M. G. S. Yap, Recombinant Protein Therapeutics from CHO Cells — 20 Years and Counting, CEP Magazine (2007) 40-47.

[30] J. X. Zhou, T. Tressel, X. Yang, T. Seewoester, Implementation of advanced technologies in commercial monoclonal antibody production, Biotechnology Journal 3 (9-10) (2008) $1185-1200$.

[31] F. Meuwly, U. Weber, T. Ziegler, A. Gervais, R. Mastrangeli, C. Crisci, M. Rossi, A. Bernard, U. von Stockar, A. Kadouri, Conversion of a CHO cell culture process from perfusion to fed-batch technology without altering product quality, Journal of Biotechnology 123 (2006) 106-116.

[32] M. S. Croughan, K. B. Konstantinov, C. Cooney, The future of industrial bioprocessing: Batch or continuous?, Biotechnology and Bioengineering 112 (4) (2015) 648-651.

[33] F. Li, N. Vijayasankaran, A. Y. Shen, R. Kiss, A. Amanullah, Cell culture processes for monoclonal antibody production, mAbs 2 (5) (2010) 466-479.

[34] Y.-M. Huang, W. Hu, E. Rustandi, K. Chang, H. Yusuf-Makagiansar, T. Ryll, Maximizing productivity of $\mathrm{CHO}$ cell-based fed-batch culture using chemically defined media conditions and typical manufacturing equipment, Biotechnology Progress 26 (5) (2010) $1400-1410$.

[35] M. Pohlscheidt, M. Jacobs, S. Wolf, J. Thiele, A. Jockwer, J. Gabelsberger, M. Jenzsch, H. Tebbe, J. Burg, Optimizing capacity utilization by large scale $3000 \mathrm{~L}$ perfusion in seed train bioreactors, Biotechnology Progress 29 (1) (2013) 222-229.

[36] D. J. Karst, E. Serra, T. K. Villiger, M. Soos, M. Morbidelli, Characterization and comparison of ATF and TFF in stirred bioreactors for continuous mammalian cell culture processes, Biochemical Engineering Journal 110 (2016) 17-26. 
[37] D. J. Karst, F. Steinebach, M. Soos, M. Morbidelli, Process performance and product quality in an integrated continuous antibody production process, Biotechnology and Bioengineering 114 (2) (2017) 298-307.

[38] K. Konstantinov, C. Goudar, M. Ng, R. Meneses, J. Thrift, S. Chuppa, C. Matanguihan, J. Michaels, D. Naveh, The "Push-to-Low" Approach for Optimization of High-Density Perfusion Cultures of Animal Cells, in: Cell Culture Engineering, no. July, 2006, pp. 75-98.

[39] N. Jenkins, P. Meleady, R. Tyther, L. Murphy, Strategies for analysing and improving the expression and quality of recombinant proteins made in mammalian cells, Biotechnology and Applied Biochemistry 53 (Pt 2) (2009) 73-83.

[40] C. A. Boswell, D. B. Tesar, K. Mukhyala, F.-P. Theil, P. J. Fielder, L. A. Khawli, Effects of charge on antibody tissue distribution and pharmacokinetics, Bioconjugate Chemistry 21 (12) (2010) 2153-2163.

[41] M. Tsubaki, I. Terashima, K. Kamata, A. Koga, C-terminal modification of monoclonal antibody drugs: amidated species as a general product-related substance, International Journal of Biological Macromolecules 52 (2013) 139-147.

[42] M. Satoh, S. Iida, Non-fucosylated therapeutic antibodies as next-generation antibodies, Expert Opinion on Biological Therapy 6 (11) (2006) 1161-1173.

[43] W. Wang, E. Q. Wang, J. P. Balthasar, Monoclonal antibody pharmacokinetics and pharmacodynamics, Clinical Pharmacology and Therapeutics 84 (5) (2008) 548-558.

[44] L. A. Khawli, S. Goswami, R. Hutchinson, Z. W. Kwong, J. Yang, X. Wang, Z. Yao, A. Sreedhara, T. Cano, D. Tesar, I. Nijem, D. E. Allison, P. Y. Wong, Y.-H. Kao, C. Quan, A. Joshi, R. J. Harris, P. Motchnik, Charge variants in IgG1: Isolation, characterization, in vitro binding properties and pharmacokinetics in rats, mAbs 2 (6) (2010) 613-624.

[45] B. Kremkow, K. Lee, Next-generation sequencing technologies and their potential impact on CHO cell-based biomanufacturing, Pharmaceutical Bioprocessing 1 (5) (2013) 455465.

[46] M. E. M. Cromwell, E. Hilario, F. Jacobson, Protein aggregation and bioprocessing, The AAPS Journal 8 (3) (2006) E572-E579.

[47] P. Bhoskar, B. Belongia, R. Smith, S. Yoon, T. Carter, J. Xu, Free light chain content in culture media reflects recombinant monoclonal antibody productivity and quality, Biotechnology Progress 29 (5) (2013) 1131-1139.

[48] A. Beck, J. M. Reichert, Marketing approval of mogamulizumab, mAbs 4 (4) (2012) 419-425.

[49] Z. Du, D. Treiber, J. D. McCarter, D. Fomina-Yadlin, R. A. Saleem, R. E. McCoy, Y. Zhang, T. Tharmalingam, M. Leith, B. D. Follstad, B. Dell, B. Grisim, C. Zupke, C. Heath, A. E. 
Morris, P. Reddy, Use of a small molecule cell cycle inhibitor to control cell growth and improve specific productivity and product quality of recombinant proteins in CHO cell cultures, Biotechnology and Bioengineering 112 (2015) 141-155.

[50] A. E. Schmelzer, W. M. Miller, Hyperosmotic stress and elevated pCO2 alter monoclonal antibody charge distribution and monosaccharide content, Biotechnology Progress 18 (2) (2002) 346-353.

[51] M. J. Gramer, Product quality considerations for Mammalian cell culture process development and manufacturing, Advances in Biochemical Engineering/Biotechnology 139 (2014) 123-166.

[52] M. J. Gramer, J. J. Eckblad, R. Donahue, J. Brown, C. Shultz, K. Vickerman, P. Priem, E. T. J. van den Bremer, J. Gerritsen, P. H. C. van Berkel, Modulation of antibody galactosylation through feeding of uridine, manganese chloride, and galactose, Biotechnology and Bioengineering 108 (7) (2011) 1591-1602.

[53] J. Luo, J. Zhang, D. Ren, W.-L. Tsai, F. Li, A. Amanullah, T. Hudson, Probing of C-terminal lysine variation in a recombinant monoclonal antibody production using Chinese hamster ovary cells with chemically defined media, Biotechnology and Bioengineering 109 (9) (2012) 2306-2315.

[54] Y. Jing, M. Borys, S. Nayak, S. Egan, Y. Qian, S.-H. Pan, Z. J. Li, Identification of cell culture conditions to control protein aggregation of IgG fusion proteins expressed in Chinese hamster ovary cells, Process Biochemistry 47 (1) (2012) 69-75.

[55] M. Trexler-Schmidt, S. Sargis, J. Chiu, S. Sze-Khoo, M. Mun, Y.-H. Kao, M. W. Laird, Identification and prevention of antibody disulfide bond reduction during cell culture manufacturing, Biotechnology and Bioengineering 106 (3) (2010) 452-461.

[56] R. J. Kaufman, M. Swaroop, P. Murtha-Riel, Depletion of manganese within the secretory pathway inhibits O-linked glycosylation in mammalian cells, Biochemistry 33 (33) (1994) 9813-9819.

[57] H. Eagle, Nutrition needs of mammalian cells in tissue culture, Science 122 (3168) (1955) 501-514.

[58] A. G. McAtee, N. Templeton, J. D. Young, Role of Chinese hamster ovary central carbon metabolism in controlling the quality of secreted biotherapeutic proteins, Pharmaceutical Bioprocessing 2 (1) (2014) 63-74.

[59] S. A. Brooks, Protein glycosylation in diverse cell systems: implications for modification and analysis of recombinant proteins, Expert Review of Proteomics 3 (3) (2006) 345-359.

[60] R. Apweiler, H. Hermjakob, N. Sharon, On the frequency of protein glycosylation, as deduced from analysis of the SWISS-PROT database, Biochimica et Biophysica Acta 1473 (1) (1999) 4-8. 
[61] H. Liu, G. Gaza-Bulseco, D. Faldu, C. Chumsae, J. Sun, Heterogeneity of Monoclonal Antibodies, Journal of Pharmaceutical Sciences 97 (7) (2008) 2426-2447.

[62] D. Chee Furng Wong, K. Tin Kam Wong, L. Tang Goh, C. Kiat Heng, M. Gek Sim Yap, Impact of dynamic online fed-batch strategies on metabolism, productivity and $\mathrm{N}$ glycosylation quality in CHO cell cultures, Biotechnology and Bioengineering 89 (2) (2005) 164-177.

[63] E. Pacis, M. Yu, J. Autsen, R. Bayer, F. Li, Effects of cell culture conditions on antibody N-linked glycosylation-what affects high mannose 5 glycoform, Biotechnology and Bioengineering 108 (10) (2011) 2348-2358.

[64] Y. Rouiller, A. Périlleux, M. Marsaut, M. Stettler, M.-N. Vesin, H. Broly, Effect of hydrocortisone on the production and glycosylation of an Fc-fusion protein in $\mathrm{CHO}$ cell cultures, Biotechnology Progress 28 (3) (2012) 803-813.

[65] Y. Konno, Y. Kobayashi, K. Takahashi, E. Takahashi, S. Sakae, M. Wakitani, K. Yamano, T. Suzawa, K. Yano, T. Ohta, M. Koike, K. Wakamatsu, S. Hosoi, Fucose content of monoclonal antibodies can be controlled by culture medium osmolality for high antibodydependent cellular cytotoxicity, Cytotechnology 64 (3) (2012) 249-265.

[66] J.-M. Yang, J. Ai, Y. Bao, Z. Yuan, Y. Qin, Y.-W. Xie, D. Tao, D. Fu, Y. Peng, Investigation of the correlation between charge and glycosylation of IgG1 variants by liquid chromatography-mass spectrometry, Analytical Biochemistry 448 (2014) 82-91.

[67] R. Jefferis, Glycosylation as a strategy to improve antibody-based therapeutics, Nature Reviews Drug Discovery 8 (3) (2009) 226-234.

[68] A. Eon-Duval, H. Broly, R. Gleixner, Quality attributes of recombinant therapeutic proteins: an assessment of impact on safety and efficacy as part of a quality by design development approach, Biotechnology Progress 28 (3) (2012) 608-622.

[69] P. Zhang, S. Woen, T. Wang, B. Liau, S. Zhao, C. Chen, Y. Yang, Z. Song, M. R. Wormald, C. Yu, P. M. Rudd, Challenges of glycosylation analysis and control: An integrated approach to producing optimal and consistent therapeutic drugs, Drug Discovery Today 21 (5) (2016) 740-765.

[70] I. J. del Val, C. Kontoravdi, J. M. Nagy, Towards the implementation of quality by design to the production of therapeutic monoclonal antibodies with desired glycosylation patterns, Biotechnology Progress 26 (6) (2010) 1505-1527.

[71] J. N. Arnold, M. R. Wormald, R. B. Sim, P. M. Rudd, R. A. Dwek, The impact of glycosylation on the biological function and structure of human immunoglobulins, Annual Review of Immunology 25 (2007) 21-50.

[72] I. H. Yuk, B. Zhang, Y. Yang, G. Dutina, K. D. Leach, N. Vijayasankaran, A. Y. Shen, D. C. Andersen, B. R. Snedecor, J. C. Joly, Controlling glycation of recombinant antibody in fed-batch cell cultures, Biotechnology and Bioengineering 108 (11) (2011) 2600-2610. 
[73] A. Lapolla, P. Traldi, D. Fedele, Importance of measuring products of non-enzymatic glycation of proteins, Clinical Biochemistry 38 (2) (2005) 103-115.

[74] X. Zhuang, X. Pang, W. Zhang, W. Wu, J. Zhao, H. Yang, W. Qu, Effects of zinc and manganese on advanced glycation end products (AGEs) formation and AGEs-mediated endothelial cell dysfunction, Life Sciences 90 (3-4) (2012) 131-139.

[75] C. Quan, E. Alcala, I. Petkovska, D. Matthews, E. Canova-Davis, R. Taticek, S. Ma, A study in glycation of a therapeutic recombinant humanized monoclonal antibody: where it is, how it got there, and how it affects charge-based behavior, Analytical Biochemistry 373 (2) (2008) 179-191.

[76] K. Nakajou, H. Watanabe, U. Kragh-Hansen, T. Maruyama, M. Otagiri, The effect of glycation on the structure, function and biological fate of human serum albumin as revealed by recombinant mutants, Biochimica et Biophysica Acta 1623 (2-3) (2003) 88-97.

[77] M. Gagnon, G. Hiller, Y.-T. Luan, A. Kittredge, J. DeFelice, D. Drapeau, High-end pHcontrolled delivery of glucose effectively suppresses lactate accumulation in CHO fedbatch cultures, Biotechnology and Bioengineering 108 (6) (2011) 1328-1337.

[78] D. Jayme, T. Watanabe, T. Shimada, Basal medium development for serum-free culture: a historical perspective, Cytotechnology 23 (1-3) (1997) 95-101.

[79] G. B. Nyberg, R. R. Balcarcel, B. D. Follstad, G. Stephanopoulos, D. I. Wang, Metabolic effects on recombinant interferon-gamma glycosylation in continuous culture of Chinese hamster ovary cells, Biotechnology and Bioengineering 62 (3) (1999) 336-347.

[80] A. E. Chapman, J. C. Calhoun, Effects of glucose starvation and puromycin treatment on lipid-linked oligosaccharide precursors and biosynthetic enzymes in Chinese hamster ovary cells in vivo and in vitro, Archives of Biochemistry and Biophysics 260 (1) (1988) 320-333.

[81] B. Liu, M. Spearman, J. Doering, E. Lattová, H. Perreault, M. Butler, The availability of glucose to CHO cells affects the intracellular lipid-linked oligosaccharide distribution, site occupancy and the $\mathrm{N}$-glycosylation profile of a monoclonal antibody, Journal of Biotechnology 170 (2014) 17-27.

[82] J. S. Seo, B. S. Min, Y. J. Kim, J. M. Cho, E. Baek, M. S. Cho, G. M. Lee, Effect of glucose feeding on the glycosylation quality of antibody produced by a human cell line, F2N78, in fed-batch culture, Applied Microbiology and Biotechnology 98 (8) (2014) 3509-3515.

[83] C. Villacrés, V. S. Tayi, E. Lattová, H. Perreault, M. Butler, Low glucose depletes glycan precursors, reduces site occupancy and galactosylation of a monoclonal antibody in CHO cell culture, Biotechnology Journal 10 (7) (2015) 1051-1066. 
[84] H. J. Cruz, C. M. Peixoto, M. Nimtz, P. M. Alves, E. M. Dias, J. L. Moreira, M. J. Carrondo, Metabolic shifts do not influence the glycosylation patterns of a recombinant fusion protein expressed in BHK cells, Biotechnology and Bioengineering 69 (2) (2000) 129-139.

[85] P. M. Hayter, E. M. Curling, A. J. Baines, N. Jenkins, I. Salmon, P. G. Strange, J. M. Tong, A. T. Bull, Glucose-limited chemostat culture of Chinese hamster ovary cells producing recombinant human interferon-gamma, Biotechnology and Bioengineering 39 (3) (1992) 327-335.

[86] N. S. C. Wong, L. Wati, P. M. Nissom, H. T. Feng, M. M. Lee, M. G. S. Yap, An investigation of intracellular glycosylation activities in $\mathrm{CHO}$ cells: effects of nucleotide sugar precursor feeding, Biotechnology and Bioengineering 107 (2) (2010) 321-336.

[87] H. Kayakiri, S. Takase, T. Shibata, M. Okamoto, H. Terano, M. Hashimoto, T. Tada, S. Koda, Structure of kifunensine, a new immunomodulator isolated from an actinomycete, The Journal of Organic Chemistry 54 (17) (1989) 4015-4016.

[88] A. D. Elbein, J. E. Tropea, M. Mitchell, G. P. Kaushal, Kifunensine, a potent inhibitor of the glycoprotein processing mannosidase I, The Journal of Biological Chemistry 265 (26) (1990) 15599-15605.

[89] E. Liebminger, S. Hüttner, U. Vavra, R. Fischl, J. Schoberer, J. Grass, C. Blaukopf, G. J. Seifert, F. Altmann, L. Mach, R. Strasser, Class I alpha-mannosidases are required for Nglycan processing and root development in Arabidopsis thaliana, The Plant Cell 21 (12) (2009) 3850-3867.

[90] T. D. Butters, D. S. Alonzi, N. V. Kukushkin, Y. Ren, Y. Blériot, Novel mannosidase inhibitors probe glycoprotein degradation pathways in cells, Glycoconjugate Journal 26 (9) (2009) 1109-1116.

[91] Q. Zhou, S. Shankara, A. Roy, H. Qiu, S. Estes, A. McVie-Wylie, K. Culm-Merdek, A. Park, C. Pan, T. Edmunds, Development of a simple and rapid method for producing nonfucosylated oligomannose containing antibodies with increased effector function, Biotechnology and Bioengineering 99 (3) (2008) 652-665.

[92] H. H. Shi, C. T. Goudar, Recent advances in the understanding of biological implications and modulation methodologies of monoclonal antibody N-linked high mannose glycans, Biotechnology and Bioengineering 111 (10) (2014) 1907-1919.

[93] A. Kato, N. Kato, E. Kano, I. Adachi, K. Ikeda, L. Yu, T. Okamoto, Y. Banba, H. Ouchi, H. Takahata, N. Asano, Biological properties of D- and L-1-deoxyazasugars, Journal of Medicinal Chemistry 48 (6) (2005) 2036-2044.

[94] K. S. Shashidhara, S. M. Gaikwad, M. I. Khan, K. C. Bharadwaj, G. Pandey, Interaction of $\alpha$-mannosidase from Aspergillus fischeri with glycosidase inhibitors, metal ions and group specific reagents, Research Journal of Biotechnology 4 (4) (2009) 39-48. 
[95] A. Vidyasagar, K. M. Sureshan, Total Synthesis and Glycosidase Inhibition Studies of (-)-Gabosine J and Its Derivatives, European Journal of Organic Chemistry 2014 (11) (2014) 2349-2356.

[96] A. Kato, L. Wang, K. Ishii, J. Seino, N. Asano, T. Suzuki, Calystegine B3 as a specific inhibitor for cytoplasmic alpha-mannosidase, Man2C1, Journal of Biochemistry 149 (4) (2011) 415-422.

[97] A. Gholamhoseinian, H. Fallah, F. Sharifi-Far, M. Mirtajaddini, Alpha Mannosidase Inhibitory Effect of Some Iranian Plant Extracts, International Journal of Pharmacology 4 (6) (2008) 460-465.

[98] Y. Zhu, M. D. L. Suits, A. J. Thompson, S. Chavan, Z. Dinev, C. Dumon, N. Smith, K. W. Moremen, Y. Xiang, A. Siriwardena, S. J. Williams, H. J. Gilbert, G. J. Davies, Mechanistic insights into a Ca2+-dependent family of alpha-mannosidases in a human gut symbiont, Nature Chemical Biology 6 (2) (2010) 125-132.

[99] R. J. Williams, J. Iglesias-Fernández, J. Stepper, A. Jackson, A. J. Thompson, E. C. Lowe, J. M. White, H. J. Gilbert, C. Rovira, G. J. Davies, S. J. Williams, Combined inhibitor freeenergy landscape and structural analysis reports on the mannosidase conformational coordinate, Angewandte Chemie (International Edition in English) 53 (4) (2014) 10871091.

[100] T. Aoyagi, T. Yamamoto, K. Kojiri, H. Morishima, M. Nagai, M. Hamada, T. Takeuchi, H. Umezawa, Mannostatins A and B: new inhibitors of alpha-D-mannosidase, produced by Streptoverticillium verticillus var. quintum ME3-AG3: taxonomy, production, isolation, physico-chemical properties and biological activities, The Journal of Antibiotics 42 (6) (1989) 883-889.

[101] D. A. Kuntz, W. Zhong, J. Guo, D. R. Rose, G.-J. Boons, The molecular basis of inhibition of Golgi alpha-mannosidase II by mannostatin A, Chembiochem 10 (2) (2009) 268-277.

[102] L. Petersen, A. Ardèvol, C. Rovira, P. J. Reilly, Molecular mechanism of the glycosylation step catalyzed by Golgi alpha-mannosidase II: a QM/MM metadynamics investigation, Journal of the American Chemical Society 132 (24) (2010) 8291-8300.

[103] C. A. Retamal, A. J. B. Dias, F. C. Brasil, F. R. Lanzana, M. L. López, Alpha-mannosidase activity in stallion epididymal fluid and spermatozoa, Theriogenology 78 (2) (2012) 252-262.

[104] N. Asano, R. J. Nash, R. J. Molyneux, G. W. Fleet, Sugar-mimic glycosidase inhibitors: natural occurrence, biological activity and prospects for therapeutic application, Tetrahedron: Asymmetry 11 (8) (2000) 1645-1680.

[105] T. Siadak, E. Espling, J. Mcgourty, R. Lowe, P. Baum, A. Wahl, P. Thompson, V. Yabannavar, Enhancing Biological Activity of Immunoglycoproteins by a Convenient Method of Generating Preferred Glycovariants (2008). 
[106] M. M. St Amand, D. Radhakrishnan, A. S. Robinson, B. A. Ogunnaike, Identification of manipulated variables for a glycosylation control strategy, Biotechnology and Bioengineering 111 (10) (2014) 1957-1970.

[107] T. Surve, M. Gadgil, Manganese increases high mannose glycoform on monoclonal antibody expressed in $\mathrm{CHO}$ when glucose is absent or limiting: Implications for use of alternate sugars, Biotechnology Progress 31 (2) (2015) 460-467.

[108] P. Hossler, S. McDermott, C. Racicot, C. Chumsae, H. Raharimampionona, Y. Zhou, D. Ouellette, J. Matuck, I. Correia, J. Fann, J. Li, Cell culture media supplementation of uncommonly used sugars sucrose and tagatose for the targeted shifting of protein glycosylation profiles of recombinant protein therapeutics, Biotechnology Progress (2014) 20-24.

[109] C.-J. Huang, H. Lin, J. X. Yang, A robust method for increasing Fc glycan high mannose level of recombinant antibodies, Biotechnology and Bioengineering 112 (6) (2015) 1200-1209.

[110] P. G. Slade, R. G. Caspary, S. Nargund, C.-J. Huang, Mannose metabolism in recombinant CHO cells and its effect on IgG glycosylation, Biotechnology and Bioengineering 113 (7) (2016) 1468-1480.

[111] Z. Tu, Y.-N. Lin, C.-H. Lin, Development of fucosyltransferase and fucosidase inhibitors, Chemical Society Reviews 42 (10) (2013) 4459-4475.

[112] C. D. Rillahan, A. Antonopoulos, C. T. Lefort, R. Sonon, P. Azadi, K. Ley, A. Dell, S. M. Haslam, J. C. Paulson, Global metabolic inhibitors of sialyl- and fucosyltransferases remodel the glycome, Nature Chemical Biology 8 (7) (2012) 661-668.

[113] J. Kamińska, J. Dziecioł, J. Kościelak, Triazine dyes as inhibitors and affinity ligands of glycosyltransferases, Glycoconjugate Journal 16 (11) (1999) 719-723.

[114] J. G. Allen, M. Mujacic, M. J. Frohn, A. J. Pickrell, P. Kodama, D. Bagal, T. San Miguel, E. A. Sickmier, S. Osgood, A. Swietlow, V. Li, J. B. Jordan, K. W. Kim, A. M. C. Rousseau, Y. J. Kim, S. Caille, M. Achmatowicz, O. Thiel, C. H. Fotsch, P. Reddy, J. D. McCarter, Facile Modulation of Antibody Fucosylation with Small Molecule Fucostatin Inhibitors and Cocrystal Structure with GDP-Mannose 4,6-Dehydratase, ACS Chemical Biology 11 (10) (2016) 2734-2743.

[115] K. Hosoguchi, T. Maeda, J.-I. Furukawa, Y. Shinohara, H. Hinou, M. Sekiguchi, H. Togame, H. Takemoto, H. Kondo, S.-I. Nishimura, An efficient approach to the discovery of potent inhibitors against glycosyltransferases, Journal of Medicinal Chemistry 53 (15) (2010) 5607-5619.

[116] X. Niu, X. Fan, J. Sun, P. Ting, S. Narula, D. Lundell, Inhibition of fucosyltransferase VII by gallic acid and its derivatives, Archives of Biochemistry and Biophysics 425 (1) (2004) 51-57. 
[117] S. C. Burleigh, T. van de Laar, C. J. M. Stroop, W. M. J. van Grunsven, N. O’Donoghue, P. M. Rudd, G. P. Davey, Synergizing metabolic flux analysis and nucleotide sugar metabolism to understand the control of glycosylation of recombinant protein in CHO cells, BMC Biotechnology 11 (1) (2011) 95.

[118] M. P. Kötzler, S. Blank, F. I. Bantleon, M. Wienke, E. Spillner, B. Meyer, Donor assists acceptor binding and catalysis of human $\alpha 1$,6-fucosyltransferase, ACS Chemical Biology 8 (8) (2013) 1830-1840.

[119] A. Zhang, V. L. Tsang, L. R. Markely, L. Kurt, Y.-M. Huang, S. Prajapati, R. Kshirsagar, Identifying the differences in mechanisms of mycophenolic acid controlling fucose content of glycoproteins expressed in different CHO cell lines, Biotechnology and Bioengineering 113 (11) (2016) 2367-2376.

[120] M. C. Galan, A. P. Venot, G.-J. Boons, Glycosyltransferase activity can be modulated by small conformational changes of acceptor substrates, Biochemistry 42 (28) (2003) 8522-8529.

[121] M. Crispin, D. J. Harvey, V. T. Chang, C. Yu, A. R. Aricescu, E. Y. Jones, S. J. Davis, R. A. Dwek, P. M. Rudd, Inhibition of hybrid- and complex-type glycosylation reveals the presence of the GlcNAc transferase I-independent fucosylation pathway, Glycobiology 16 (8) (2006) 748-756.

[122] R. K. Grainger, D. C. James, CHO cell line specific prediction and control of recombinant monoclonal antibody N-glycosylation, Biotechnology and Bioengineering 110 (11) (2013) 2970-2983.

[123] A. E. Hills, A. Patel, P. Boyd, D. C. James, Metabolic control of recombinant monoclonal antibody N-glycosylation in GS-NS0 cells, Biotechnology and Bioengineering 75 (2) (2001) 239-251.

[124] H. F. Kildegaard, Y. Fan, J. W. Sen, B. Larsen, M. R. Andersen, Glycoprofiling effects of media additives on IgG produced by $\mathrm{CHO}$ cells in fed-batch bioreactors, Biotechnology and Bioengineering 113 (2) (2016) 359-366.

[125] N. A. McCracken, R. Kowle, A. Ouyang, Control of galactosylated glycoforms distribution in cell culture system, Biotechnology Progress 30 (3) (2014) 547-553.

[126] P. Chen, S. W. Harcum, Effects of elevated ammonium on glycosylation gene expression in CHO cells, Metabolic Engineering 8 (2) (2006) 123-132.

[127] K. Descroix, G. K. Wagner, The first C-glycosidic analogue of a novel galactosyltransferase inhibitor, Organic \& Biomolecular Chemistry 9 (6) (2011) 1855-1863.

[128] K. Descroix, T. Pesnot, Y. Yoshimura, S. S. Gehrke, W. Wakarchuk, M. M. Palcic, G. K. Wagner, Inhibition of galactosyltransferases by a novel class of donor analogues, Journal of Medicinal Chemistry 55 (5) (2012) 2015-2024. 
[129] J. R. Brown, F. Yang, A. Sinha, B. Ramakrishnan, Y. Tor, P. K. Qasba, J. D. Esko, Deoxygenated disaccharide analogs as specific inhibitors of betal-4-galactosyltransferase 1 and selectin-mediated tumor metastasis, The Journal of Biological Chemistry 284 (8) (2009) 4952-4959.

[130] N. Mitsuhashi, H. Yuasa, A Novel Galactosyltransferase Inhibitor with Diamino Sugar as a Pyrophosphate Mimic, European Journal of Organic Chemistry 2009 (10) (2009) 1598-1605.

[131] Y. Gao, J. Z. Vlahakis, W. A. Szarek, I. Brockhausen, Selective inhibition of glycosyltransferases by bivalent imidazolium salts, Bioorganic \& Medicinal Chemistry 21 (5) (2013) 1305-1311.

[132] Y. Gao, C. Lazar, W. A. Szarek, I. Brockhausen, Specificity of $\beta 1$,4-galactosyltransferase inhibition by 2-naphthyl 2-butanamido-2-deoxy-1-thio- $\beta$-D-glucopyranoside, Glycoconjugate Journal 27 (7-9) (2010) 673-684.

[133] T. Hayashi, B. W. Murray, R. Wang, C. H. Wong, A chemoenzymatic synthesis of UDP-(2deoxy-2-fluoro)-galactose and evaluation of its interaction with galactosyltransferase, Bioorganic \& Medicinal Chemistry 5 (3) (1997) 497-500.

[134] X. Gu, D. I. Wang, Improvement of interferon-gamma sialylation in Chinese hamster ovary cell culture by feeding of $\mathrm{N}$-acetylmannosamine, Biotechnology and Bioengineering 58 (6) (1998) 642-648.

[135] K. N. Baker, M. H. Rendall, A. E. Hills, M. Hoare, R. B. Freedman, D. C. James, Metabolic control of recombinant protein $\mathrm{N}$-glycan processing in NS0 and CHO cells, Biotechnology and Bioengineering 73 (3) (2001) 188-202.

[136] K. Bork, W. Reutter, W. Weidemann, R. Horstkorte, Enhanced sialylation of EPO by overexpression of UDP-GlcNAc 2-epimerase/ManAc kinase containing a sialuria mutation in CHO cells, FEBS Letters 581 (22) (2007) 4195-4198.

[137] K. Bork, R. Horstkorte, W. Weidemann, Increasing the sialylation of therapeutic glycoproteins: the potential of the sialic acid biosynthetic pathway, Journal of Pharmaceutical Sciences 98 (10) (2009) 3499-3508.

[138] M. B. Jones, H. Teng, J. K. Rhee, N. Lahar, G. Baskaran, K. J. Yarema, Characterization of the cellular uptake and metabolic conversion of acetylated $\mathrm{N}$-acetylmannosamine (ManNAc) analogues to sialic acids, Biotechnology and Bioengineering 85 (4) (2004) 394-405.

[139] J. Rodriguez, M. Spearman, N. Huzel, M. Butler, Enhanced production of monomeric interferon-beta by $\mathrm{CHO}$ cells through the control of culture conditions, Biotechnology Progress 21 (1) (2005) 22-30. 
[140] J. Liu, J. Wang, L. Fan, X. Chen, D. Hu, X. Deng, H. Fai Poon, H. Wang, X. Liu, W. S. Tan, Galactose supplementation enhance sialylation of recombinant $\mathrm{Fc}$-fusion protein in CHO cell: an insight into the role of galactosylation in sialylation, World Journal of Microbiology and Biotechnology 31 (7) (2015) 1147-1156.

[141] M. Yang, M. Butler, Effects of ammonia and glucosamine on the heterogeneity of erythropoietin glycoforms, Biotechnology Progress 18 (1) (2002) 129-138.

[142] S. Magesh, V. Savita, S. Moriya, T. Suzuki, T. Miyagi, H. Ishida, M. Kiso, Human sialidase inhibitors: design, synthesis, and biological evaluation of 4-acetamido-5-acylamido-2fluoro benzoic acids, Bioorganic \& Medicinal Chemistry 17 (13) (2009) 4595-4603.

[143] T. K. Ha, Y.-G. Kim, G. M. Lee, Effect of lithium chloride on the production and sialylation of Fc-fusion protein in Chinese hamster ovary cell culture, Applied Microbiology and Biotechnology 98 (22) (2014) 9239-9248.

[144] A. Viktorínová, E. Toserová, M. Krizko, Z. Duracková, Altered metabolism of copper, zinc, and magnesium is associated with increased levels of glycated hemoglobin in patients with diabetes mellitus, Metabolism: Clinical and Experimental 58 (10) (2009) 1477-1482.

[145] K. Mikulíková, A. Eckhardt, J. Kunes, J. Zicha, I. Miksík, Advanced glycation end-product pentosidine accumulates in various tissues of rats with high fructose intake, Physiological research / Academia Scientiarum Bohemoslovaca 57 (1) (2008) 89-94.

[146] J. Han, C. Tan, Y. Wang, S. Yang, D. Tan, Betanin reduces the accumulation and cross-links of collagen in high-fructose-fed rat heart through inhibiting non-enzymatic glycation, Chemico-Biological Interactions 227C (2015) 37-44.

[147] C.-H. Wu, S.-M. Huang, J.-A. Lin, G.-C. Yen, Inhibition of advanced glycation endproduct formation by foodstuffs, Food \& Function 2 (5) (2011) 224-234.

[148] G. Gaza-Bulseco, B. Li, A. Bulseco, H. C. Liu, Method to differentiate asn deamidation that occurred prior to and during sample preparation of a monoclonal antibody, Analytical Chemistry 80 (24) (2008) 9491-9498.

[149] J. Vlasak, R. Ionescu, Heterogeneity of Monoclonal Antibodies Revealed by ChargeSensitive Methods, Current Pharmaceutical Biotechnology 9 (6) (2008) 468-481.

[150] H. Yang, R. A. Zubarev, Mass spectrometric analysis of asparagine deamidation and aspartate isomerization in polypeptides, Electrophoresis 31 (11) (2010) 1764-1772.

[151] A. L. Pace, R. L. Wong, Y. T. Zhang, Y.-H. Kao, Y. J. Wang, Asparagine deamidation dependence on buffer type, pH, and temperature, Journal of Pharmaceutical Sciences 102 (6) (2013) 1712-1723.

[152] L. P. Stratton, R. M. Kelly, J. Rowe, J. E. Shively, D. D. Smith, J. F. Carpenter, M. C. Manning, Controlling deamidation rates in a model peptide: effects of temperature, peptide 
concentration, and additives, Journal of Pharmaceutical Sciences 90 (12) (2001) 21412148 .

[153] A. A. Wakankar, R. T. Borchardt, Formulation Considerations for Proteins Susceptible to Asparagine Deamidation and Aspartate Isomerization, Journal of Pharmaceutical Sciences 95 (11) (2006) 2321-2336.

[154] J. A. Ji, B. Zhang, W. Cheng, Y. J. Wang, Methionine, tryptophan, and histidine oxidation in a model protein, PTH: mechanisms and stabilization, Journal of Pharmaceutical Sciences 98 (12) (2009) 4485-4500.

[155] J. Bergès, P. Trouillas, C. Houée-Levin, Oxidation of protein tyrosine or methionine residues: From the amino acid to the peptide, Journal of Physics: Conference Series 261 (2011) 012003.

[156] E. Folzer, K. Diepold, K. Bomans, C. Finkler, R. Schmidt, P. Bulau, J. Huwyler, H. C. Mahler, A. V. Koulov, Selective Oxidation of Methionine and Tryptophan Residues in a Therapeutic IgG1 Molecule, Journal of Pharmaceutical Sciences 104 (9) (2015) 28242831.

[157] S. Li, T. H. Nguyen, C. Schöneich, R. T. Borchardt, Aggregation and precipitation of human relaxin induced by metal-catalyzed oxidation, Biochemistry 34 (17) (1995) 57625772 .

[158] L. W. Dick, D. Qiu, D. Mahon, M. Adamo, K.-C. Cheng, C-terminal lysine variants in fully human monoclonal antibodies: investigation of test methods and possible causes, Biotechnology and Bioengineering 100 (6) (2008) 1132-1143.

[159] W. Xu, Y. Peng, F. Wang, B. Paporello, D. Richardson, H. Liu, Method to convert Nterminal glutamine to pyroglutamate for characterization of recombinant monoclonal antibodies, Analytical Biochemistry 436 (1) (2013) 10-12.

[160] M. Schiestl, T. Stangler, C. Torella, T. Cepeljnik, H. Toll, R. Grau, Acceptable changes in quality attributes of glycosylated biopharmaceuticals, Nature Biotechnology 29 (4) (2011) 310-312.

[161] K. A. Johnson, K. Paisley-Flango, B. S. Tangarone, T. J. Porter, J. C. Rouse, Cation exchangeHPLC and mass spectrometry reveal C-terminal amidation of an IgG1 heavy chain, Analytical Biochemistry 360 (1) (2007) 75-83.

[162] T. Kaschak, D. Boyd, F. Lu, G. Derfus, B. Kluck, B. Nogal, C. Emery, C. Summers, K. Zheng, R. Bayer, A. Amanullah, B. Yan, Characterization of the basic charge variants of a human IgG1: effect of copper concentration in cell culture media, mAbs 3 (6) (2011) 577-583.

[163] I. H. Yuk, S. Russell, Y. Tang, W.-T. Hsu, J. B. Mauger, R. P. S. Aulakh, J. Luo, M. Gawlitzek, J. C. Joly, Effects of copper on CHO cells: Cellular requirements and product quality considerations, Biotechnology Progress (2014) 1-13. 
[164] N. Vijayasankaran, S. Varma, Y. Yang, M. Mun, S. Arevalo, M. Gawlitzek, T. Swartz, A. Lim, F. Li, B. Zhang, S. Meier, R. Kiss, Effect of cell culture medium components on color of formulated monoclonal antibody drug substance, Biotechnology Progress 29 (5) (2013) $1270-1277$.

[165] X. Zhang, H. Tang, Y. T. Sun, X. Liu, W. S. Tan, L. Fan, Elucidating the effects of arginine and lysine on a monoclonal antibody C-terminal lysine variation in $\mathrm{CHO}$ cell cultures, Applied Microbiology and Biotechnology 99 (16) (2015) 6643-6652.

[166] C. Chumsae, K. Gifford, W. Lian, H. Liu, C. H. Radziejewski, Z. S. Zhou, Arginine modifications by methylglyoxal: discovery in a recombinant monoclonal antibody and contribution to acidic species, Analytical Chemistry 85 (23) (2013) 11401-11409.

[167] P. Hossler, M. Wang, S. McDermott, C. Racicot, K. Chemfe, Y. Zhang, C. Chumsae, A. Manuilov, Cell culture media supplementation of bioflavonoids for the targeted reduction of acidic species charge variants on recombinant therapeutic proteins, Biotechnology Progress 31 (4) (2015) 1039-1052.

[168] M. Vázquez-Rey, D. A. Lang, Aggregates in monoclonal antibody manufacturing processes, Biotechnology and Bioengineering 108 (7) (2011) 1494-1508.

[169] A. J. Paul, K. Schwab, F. Hesse, Direct analysis of mAb aggregates in mammalian cell culture supernatant, BMC Biotechnology 14 (1) (2014) 99.

[170] Y.-B. Zhang, J. Howitt, S. McCorkle, P. Lawrence, K. Springer, P. Freimuth, Protein aggregation during overexpression limited by peptide extensions with large net negative charge, Protein Expression and Purification 36 (2) (2004) 207-216.

[171] M. Spearman, J. Rodriguez, N. Huzel, K. Sunley, Effect of Culture Conditions on Glycosylation of Recombinant beta-Interferon in CHO Cells, Cell Technology for Cell Products (2007) 71-85.

[172] H. K. Ju, S.-J. Hwang, C.-J. Jeon, G. M. Lee, S. K. Yoon, Use of NaCl prevents aggregation of recombinant COMP-angiopoietin-1 in Chinese hamster ovary cells, Journal of Biotechnology 143 (2) (2009) 145-150.

[173] C. R. Brown, L. Q. Hong-Brown, J. Biwersi, A. S. Verkman, W. J. Welch, Chemical chaperones correct the mutant phenotype of the delta F508 cystic fibrosis transmembrane conductance regulator protein, Cell Stress \& Chaperones 1 (2) (1996) 117-125.

[174] S. Zhou, B. Zhang, E. Sturm, D. L. Teagarden, C. Schöneich, P. Kolhe, L. M. Lewis, B. K. Muralidhara, S. K. Singh, Comparative evaluation of disodium edetate and diethylenetriaminepentaacetic acid as iron chelators to prevent metal-catalyzed destabilization of a therapeutic monoclonal antibody, Journal of Pharmaceutical Sciences 99 (10) (2010) 4239-4250. 
[175] H.-C. Mahler, W. Friess, U. Grauschopf, S. Kiese, Protein aggregation: pathways, induction factors and analysis, Journal of Pharmaceutical Sciences 98 (9) (2009) 2909-2934.

[176] P. Hossler, S. McDermott, C. Racicot, J. C. H. Fann, Improvement of mammalian cell culture performance through surfactant enabled concentrated feed media, Biotechnology Progress 29 (4) (2013) 1023-1033.

[177] M. Onitsuka, M. Tatsuzawa, R. Asano, I. Kumagai, A. Shirai, H. Maseda, T. Omasa, Trehalose suppresses antibody aggregation during the culture of Chinese hamster ovary cells, Journal of Bioscience and Bioengineering 117 (5) (2014) 632-638.

[178] M. Onitsuka, A. Kawaguchi, R. Asano, I. Kumagai, K. Honda, H. Ohtake, T. Omasa, Glycosylation analysis of an aggregated antibody produced by Chinese hamster ovary cells in bioreactor culture, Journal of Bioscience and Bioengineering 117 (5) (2014) 639-644.

[179] J. Vlasak, R. Ionescu, Fragmentation of monoclonal antibodies, mAbs 3 (3) (2011) 253-263.

[180] H. Sandberg, D. Lütkemeyer, S. Kuprin, M. Wrangel, A. Almstedt, P. Persson, V. Ek, M. Mikaelsson, Mapping and partial characterization of proteases expressed by a CHO production cell line, Biotechnology and Bioengineering 95 (5) (2006) 961-971.

[181] F. Robert, H. Bierau, M. Rossi, D. Agugiaro, T. Soranzo, H. Broly, C. Mitchell-Logean, Degradation of an Fc-fusion recombinant protein by host cell proteases: Identification of a CHO cathepsin D protease, Biotechnology and Bioengineering 104 (6) (2009) 1132-1141.

[182] S. X. Gao, Y. Zhang, K. Stansberry-Perkins, A. Buko, S. Bai, V. Nguyen, M. L. Brader, Fragmentation of a highly purified monoclonal antibody attributed to residual CHO cell protease activity, Biotechnology and Bioengineering 108 (4) (2011) 977-982.

[183] D. Ouellette, L. Alessandri, R. Piparia, A. Aikhoje, A. Chin, C. Radziejewski, I. Correia, Elevated cleavage of human immunoglobulin gamma molecules containing a lambda light chain mediated by iron and histidine, Analytical Biochemistry 389 (2) (2009) 107-117.

[184] K. Kim, S. G. Rhee, E. R. Stadtman, Nonenzymatic cleavage of proteins by reactive oxygen species generated by dithiothreitol and iron, The Journal of Biological Chemistry 260 (29) (1985) 15394-15397.

[185] W. B. Chaderjian, E. T. Chin, R. J. Harris, T. M. Etcheverry, Effect of copper sulfate on performance of a serum-free $\mathrm{CHO}$ cell culture process and the level of free thiol in the recombinant antibody expressed, Biotechnology Progress 21 (2) (2005) 550-553.

[186] Y.-H. Kao, D. P. Hewitt, M. Trexler-Schmidt, M. W. Laird, Mechanism of antibody reduction in cell culture production processes, Biotechnology and Bioengineering 107 (4) (2010) 622-632. 
[187] A. J. Cordoba, B.-J. Shyong, D. Breen, R. J. Harris, Non-enzymatic hinge region fragmentation of antibodies in solution, Journal of Chromatography B 818 (2) (2005) 115-121.

[188] B. Turk, Targeting proteases: successes, failures and future prospects, Nature Reviews. Drug Discovery 5 (9) (2006) 785-799.

[189] D. Wen, M. M. Vecchi, S. Gu, L. Su, J. Dolnikova, Y.-M. Huang, S. F. Foley, E. Garber, N. Pederson, W. Meier, Discovery and investigation of misincorporation of serine at asparagine positions in recombinant proteins expressed in Chinese hamster ovary cells, The Journal of Biological Chemistry 284 (47) (2009) 32686-32694.

[190] A. Khetan, Y.-M. Huang, J. Dolnikova, N. E. Pederson, D. Wen, H. Yusuf-Makagiansar, P. Chen, T. Ryll, Control of misincorporation of serine for asparagine during antibody production using CHO cells, Biotechnology and Bioengineering 107 (1) (2010) 116-123.

[191] H. Zhang, H. Wang, M. Liu, T. Zhang, J. Zhang, X. Wang, W. Xiang, Rational development of a serum-free medium and fed-batch process for a GS-CHO cell line expressing recombinant antibody, Cytotechnology 65 (3) (2013) 363-378.

[192] H. Galewitz (Ed.), Music - A Book of Quotations, Dover Publications, Inc., Mineaola, 2001.

[193] Y. Goto, Y. Niwa, T. Suzuki, S. Uematsu, N. Dohmae, S. Simizu, N-glycosylation is required for secretion and enzymatic activity of human hyaluronidase1, FEBS Open Bio 4 (2014) $554-559$.

[194] V. Kayser, N. Chennamsetty, V. Voynov, K. Forrer, B. Helk, B. L. Trout, Glycosylation influences on the aggregation propensity of therapeutic monoclonal antibodies, Biotechnology Journal 6 (1) (2011) 38-44.

[195] R. J. Solá, K. Griebenow, Glycosylation of Therapeutic Proteins: An Effective Strategy to Optimiza Efficacy, BioDrugs 24 (1) (2011) 9-21.

[196] R. Abès, J. L. Teillaud, Impact of glycosylation on effector functions of therapeutic IgG, Pharmaceuticals 3 (1) (2010) 146-157.

[197] Y. Kanda, T. Yamada, K. Mori, A. Okazaki, M. Inoue, K. Kitajima-Miyama, R. KuniKamochi, R. Nakano, K. Yano, S. Kakita, K. Shitara, M. Satoh, Comparison of biological activity among nonfucosylated therapeutic IgGl antibodies with three different $\mathrm{N}$-linked Fc oligosaccharides: The high-mannose, hybrid, and complex types, Glycobiology 17 (1) (2007) 104-118.

[198] B. J. Scallon, S. H. Tam, S. G. McCarthy, A. N. Cai, T. S. Raju, Higher levels of sialylated Fc glycans in immunoglobulin $\mathrm{G}$ molecules can adversely impact functionality, Molecular Immunology 44 (7) (2007) 1524-1534. 
[199] A. Wright, S. L. Morrison, Effect of C2-associated carbohydrate structure on Ig effector function: studies with chimeric mouse-human IgG1 antibodies in glycosylation mutants of Chinese hamster ovary cells, J Immunol 160 (7) (1998) 3393-3402.

[200] J. Hodoniczky, Z. Z. Yuan, D. C. James, Control of recombinant monoclonal antibody effector functions by Fc N-glycan remodeling in vitro, Biotechnology Progress 21 (6) (2005) 1644-1652.

[201] F. Nimmerjahn, J. V. Ravetch, Fc-gamma receptors as regulators of immune responses, Nature Reviews Immunology 8 (1) (2008) 34-47.

[202] D. C. F. Wong, N. S. C. Wong, J. S. Y. Goh, L. M. May, M. G. S. Yap, Profiling of Nglycosylation gene expression in CHO cell fed-batch cultures, Biotechnology and Bioengineering 107 (3) (2010) 516-528.

[203] D. Brühlmann, M. Jordan, J. Hemberger, M. Sauer, M. Stettler, H. Broly, Tailoring recombinant protein quality by rational media design, Biotechnology Progress 31 (3) (2015) 615-629.

[204] E. J. M. Blondeel, K. Braasch, T. McGill, D. Chang, C. Engel, M. Spearman, M. Butler, M. G. Aucoin, Tuning a MAb glycan profile in cell culture: Supplementing Nacetylglucosamine to favour G0 glycans without compromising productivity and cell growth, Journal of Biotechnology 214 (2015) 3.

[205] B. R. Kilgore, A. W. Lucka, R. Patel, B. A. Andrien, S. T. Dhume, Comparability and monitoring immunogenic $\mathrm{N}$-linked oligosaccharides from recombinant monoclonal antibodies from two different cell lines using HPLC with fluorescence detection and mass spectrometry, Methods in Molecular Biology 446 (2) (2008) 333-346.

[206] W. E. Stone, W. H. Baird, the Occurrence of Raffinose in American Sugar Beets, Journal of the American Chemical Society 19 (2) (1897) 116-124.

[207] A. I. Elsayed, M. S. Rafudeen, D. Golldack, Physiological aspects of raffinose family oligosaccharides in plants: Protection against abiotic stress, Plant Biology 16 (1) (2014) $1-8$.

[208] M. A. Hannah, E. Zuther, K. Buchel, A. G. Heyer, Transport and metabolism of raffinose family oligosaccharides in transgenic potato, Journal of Experimental Botany 57 (14) (2006) 3801-3811.

[209] S. Pacifici, J. Song, C. K. Zhang, E. Tako, Evaluating the effect of plant origin prebiotics (Raffinose and Stachyose) on iron status, intestinal functionality and intestinal bacterial populations in vivo, The FASEB Journal 30 (1_Supplement) (2016) 692.17.

[210] D. C. Nieman, J. Scherr, B. Luo, M. P. Meaney, D. Dréau, W. Sha, D. A. Dew, D. A. Henson, K. L. Pappan, Influence of pistachios on performance and exercise-induced inflammation, oxidative stress, immune dysfunction, and metabolite shifts in cyclists: a randomized, crossover trial, PloS one 9 (11) (2014) e113725. 
[211] W. Laroy, R. Contreras, N. Callewaert, Glycome mapping on DNA sequencing equipment, Nature Protocols 1 (1) (2006) 397-405.

[212] B. Bucsella, A. Fornage, C. L. Denmat, F. Kálmán, Nucleotide and Nucleotide Sugar Analysis in Cell Extracts by Capillary Electrophoresis, CHIMIA International Journal for Chemistry 70 (10) (2016) 732-735.

[213] bcl2fastq Conversion Software, http://support.illumina.com/sequencing/ sequencing\{_\}software/bcl2fastq-conversion-software.html.

[214] Trimmomatic, a flexible trimmer for Illumina sequence data, Bioinformatics 30 (2014) 2114.

[215] GenDBE - ProCell, https://gendbe.cebitec.uni-bielefeld.de/cho.html.

[216] TopHat - A spliced read mapper for RNA-Seq, https://ccb.jhu.edu/software/tophat/ index.shtml.

[217] Bowtie 2, http://bowtie-bio.sourceforge.net/bowtie2/index.shtml.

[218] S. Anders, P. T. Pyl, W. Huber, HTSeq-A Python framework to work with high-throughput sequencing data, Bioinformatics 31 (2) (2015) 166-169.

[219] M. I. Love, W. Huber, S. Anders, Moderated estimation of fold change and dispersion for RNA-seq data with DESeq2, Genome Biology 15 (12) (2014) 550.

[220] X. Liu, C.-C. Zhang, Z. Liu, L. Wei, Y.-J. Liu, J. Yu, L.-X. Sun, LC-based targeted metabolomics analysis of nucleotides and identification of biomarkers associated with chemotherapeutic drugs in cultured cell models, Anti-Cancer Drugs 25 (6) (2014) 1.

[221] MGAT5 mannosyl (alpha-1,6-)-glycoprotein beta-1,6-N-acetyl-glucosaminyltransferase [ Homo sapiens (human) ], https://www.ncbi.nlm.nih.gov/gene/4249.

[222] B4GALT3 beta-1,4-galactosyltransferase 3 [ Homo sapiens (human) ], https://www.ncbi. nlm.nih.gov/gene/8703.

[223] N. Ishida, M. Kawakita, Molecular physiology and pathology of the nucleotide sugar transporter family (SLC35), Pflugers Archiv European Journal of Physiology 447 (5) (2004) 768-775.

[224] P. P. Lu, O. Hindsgaul, C. A. Compston, M. M. Palcic, New synthetic trisaccharide inhibitors for $\mathrm{N}$-acetylglucosaminyltransferase-V, Bioorganic and Medicinal Chemistry 4 (11) (1996) 2011-2022.

[225] P. P. Lu, O. Hindsgaul, H. Li, M. M. Palcic, Synthesis and evaluation of eight aminodeoxy trisaccharide inhibitors for $\mathrm{N}$-acetylglucosaminyltransferase- $\mathrm{V}$, Carbohydrate Research 303 (3) (1997) 283-291. 
[226] R. B. Zavareh, Investigation of the Effects of Inhibiting N- Glycosylation in Cancer, Ph.D. thesis, University of Toronto (2011).

[227] D. Houde, Y. Peng, S. A. Berkowitz, J. R. Engen, Post-translational modifications differentially affect IgG1 conformation and receptor binding, Mol Cell Proteomics 9 (8) (2010) 1716-1728.

[228] M. Thomann, K. Reckermann, D. Reusch, J. Prasser, M. L. Tejada, Fc-galactosylation modulates antibody-dependent cellular cytotoxicity of therapeutic antibodies, Molecular Immunology 73 (2016) 69-75.

[229] A. W. Chung, M. Crispin, L. Pritchard, H. Robinson, M. K. Gorny, X. Yu, C. Bailey-Kellogg, M. E. Ackerman, C. Scanlan, S. Zolla-Pazner, G. Alter, Identification of antibody glycosylation structures that predict monoclonal antibody Fc-effector function HHS Public Access, AIDS. November 13 (2817) (2014) 2523-2530. arXiv: 15334406.

[230] T. S. Raju, Terminal sugars of Fc glycans influence antibody effector functions of IgGs, Current Opinion in Immunology 20 (4) (2008) 471-478.

[231] B. C. Jansen, A. Bondt, K. R. Reiding, E. Lonardi, C. J. de Jong, D. Falck, G. S. M. Kammeijer, R. J. E. M. Dolhain, Y. Rombouts, M. Wuhrer, Pregnancy-associated serum N-glycome changes studied by high-throughput MALDI-TOF-MS, Scientific Reports 6 (2016) 23296.

[232] M. R. Wormald, R. A. Dwek, Glycoproteins: Glycan presentation and protein-fold stability, Structure 7 (7) (1999) 155-160.

[233] R. B. Parekh, R. A. Dwek, B. J. Sutton, D. L. Fernandes, A. Leung, D. Stanworth, T. W. Rademacher, T. Mizuochi, T. Taniguchi, K. Matsuta, F. Takeuchi, Y. Nagano, T. Miyamoto, A. Kobata, Association of rheumatoid arthritis and primary osteoarthritis with changes in the glycosylation pattern of total serum IgG, Nature 316 (6027) (1985) 452-457.

[234] R. Parekh, D. A. Isenberg, B. M. Ansell, I. M. Roitt, R. A. Dwek, T. W. Rademacher, IgGassociated agalactosyl oligosaccharides, The Lancet 331 (1988) 966-969.

[235] L. R. Ruhaak, H. W. Uh, A. M. Deelder, R. E. J. M. Dolhain, M. Wuhrer, Total plasma N-glycome changes during pregnancy, Journal of Proteome Research 13 (3) (2014) 1657-1668.

[236] A. Bondt, M. H. J. Selman, A. M. Deelder, J. M. W. Hazes, S. P. Willemsen, M. Wuhrer, R. J. E. M. Dolhain, Association between galactosylation of immunoglobulin G and improvement of rheumatoid arthritis during pregnancy is independent of sialylation, Journal of Proteome Research 12 (10) (2013) 4522-4531.

[237] M. Gawlitzek, T. Ryll, J. Lofgren, M. B. Sliwkowski, Ammonium Alters N-Glycan Structures of Recombinant TNFR-IgG: Degradative Versus Biosynthetic Mechanisms, Biotechnology and Bioengineering 68 (6) (2000) 637-646. 
[238] Z. Xing, Z. Li, V. Chow, S. Lee, Identifying Inhibitory Threshold Values of Repressing Metabolites in CHO Cell Culture Using Multivariate Analysis Methods, Biotechnology Progress 24 (3) (2008) 675-683.

[239] S. Gréco, E. Niepceron, I. Hugueny, P. George, P. Louisot, M. C. Biol, Dietary spermidine and spermine participate in the maturation of galactosyltransferase activity and glycoprotein galactosylation in rat small intestine, The Journal of Nutrition 131 (7) (2001) 1890-1897.

[240] S. Mandal, A. Mandal, H. E. Johansson, A. V. Orjalo, M. H. Park, Depletion of cellular polyamines, spermidine and spermine, causes a total arrest in translation and growth in mammalian cells, Proceedings of the National Academy of Sciences of the United States of America 110 (6) (2013) 2169-2174.

[241] G. Landau, Z. Bercovich, M. H. Park, C. Kahana, The role of polyamines in supporting growth of mammalian cells is mediated through their requirement for translation initiation and elongation, The Journal of Biological Chemistry 285 (17) (2010) 12474-12481.

[242] V. Brinks, W. Jiskoot, H. Schellekens, Immunogenicity of therapeutic proteins: the use of animal models, Pharmaceutical Research 28 (10) (2011) 2379-2385.

[243] M. G. Tovey, C. Lallemand, Immunogenicity and other problems associated with the use of biopharmaceuticals, Therapeutic Advances in Drug Safety 2 (3) (2011) 113-128.

[244] S. Hermeling, D. J. A. Crommelin, H. Schellekens, W. Jiskoot, Structure-Immunogenicity Relationships of Therapeutic Proteins, Pharmaceutical Research 21 (6) (2004) 897-903.

[245] R. Jefferis, Posttranslational Modifications and the Immunogenicity of Biotherapeutics, Journal of Immunology Research 2016 (2016) 1-15.

[246] M. Baker, H. M. Reynolds, B. Lumicisi, C. J. Bryson, Immunogenicity of protein therapeutics: The key causes, consequences and challenges, Self/Nonself 1 (4) (2010) 314-322.

[247] D. M. Ecker, S. D. Jones, H. L. Levine, The therapeutic monoclonal antibody market, mAbs 7 (1) (2015) 9-14.

[248] P. Chames, M. Van Regenmortel, E. Weiss, D. Baty, Therapeutic antibodies: Successes, limitations and hopes for the future, British Journal of Pharmacology 157 (2) (2009) 220-233.

[249] Y. Ishii, J. Murakami, K. Sasaki, M. Tsukahara, K. Wakamatsu, Efficient folding/assembly in Chinese hamster ovary cells is critical for high quality (low aggregate content) of secreted trastuzumab as well as for high production: Stepwise multivariate regression analyses, Journal of Bioscience and Bioengineering 118 (2) (2014) 223-230.

[250] J. R. L. Pink, C. Milstein, Inter Heavy-Light Chain Disulphide Bridge in Immune Globulins, Nature 214 (5083) (1967) 92-94. 
[251] M. Jordan, M. Stettler, Tools for high-throughput process and medium optimization, Methods in Molecular Biology 1104 (2014) 77-88.

[252] J. R. Hart, Ethylenediaminetetraacetic Acid and Related Chelating Agents, in: Ullmann's Encyclopedia of Industrial Chemistry, Wiley-VCH Verlag GmbH \& Co. KGaA, Weinheim, Germany, 2011, pp. 547-572. arXiv: 14356007.

[253] S. Bannai, Exchange of cystine and glutamate across plasma membrane of human fibroblasts, Journal of Biological Chemistry 261 (5) (1986) 2256-2263.

[254] Y. Samuni, S. Goldstein, O. M. Dean, M. Berk, The chemistry and biological activities of $\mathrm{N}$-acetylcysteine, Biochemica et Biophysica 1830 (8) (2013) 4117-4129.

[255] J. E. Raftos, S. Whillier, B. E. Chapman, P. W. Kuchel, Kinetics of uptake and deacetylation of $\mathrm{N}$-acetylcysteine by human erythrocytes, International Journal of Biochemistry and Cell Biology 39 (9) (2007) 1698-1706.

[256] M. F. Banks, M. H. Stipanuk, The utilization of N-acetylcysteine and 2-oxothiazolidine4-carboxylate by rat hepatocytes is limited by their rate of uptake and conversion to cysteine, The Journal of Nutrition 124 (3) (1994) 378-387.

[257] P. C. Jocelyn, Chemical reduction of disulfides, Methods in Enzymology 143 (1987) 246-256.

[258] J. Nordberg, E. S. Arnér, Reactive oxygen species, antioxidants, and the mammalian thioredoxin system, Free Radical Biology and Medicine 31 (11) (2001) 1287-1312. arXiv: 10.1016.

[259] A. Holmgren, J. Lu, Thioredoxin and thioredoxin reductase: Current research with special reference to human disease, Biochemical and Biophysical Research Communications 396 (1) (2010) 120-124.

[260] C. Johansson, C. H. Lillig, A. Holmgren, Human Mitochondrial Glutaredoxin Reduces S-Glutathionylated Proteins with High Affinity Accepting Electrons from Either Glutathione or Thioredoxin Reductase, Journal of Biological Chemistry 279 (9) (2004) 7537-7543.

[261] E. C. Li, R. Abbas, I. A. Jacobs, D. Yin, Considerations in the early development of biosimilar products, Drug Discovery Today 20 (May) (2015) 1-9.

[262] R. Kumar, J. Singh, Biosimilar drugs: Current status, International Journal of Applied and Basic Medical Research 4 (2) (2014) 63.

[263] H. Schellekens, Bioequivalence and the immunogenicity of biopharmaceuticals, Nature Reviews Drug Discovery 1 (6) (2002) 457-462.

[264] H. Schellekens, J. C. Ryff, 'Biogenerics': The off-patent biotech products, Trends in Pharmacological Sciences 23 (3) (2002) 119-121. 
[265] Biosimilars approved in Europe, http://www.gabionline.net/Biosimilars/General/ Biosimilars-approved-in-Europe (2016).

[266] R. Bhambure, K. Kumar, A. S. Rathore, High-throughput process development for biopharmaceutical drug substances, Trends in Biotechnology 29 (3) (2011) 127-135.

[267] A. Amanullah, J. M. Otero, M. Mikola, A. Hsu, J. Zhang, J. Aunins, H. B. Schreyer, J. A. Hope, A. P. Russo, Novel micro-bioreactor high throughput technology for cell culture process development: Reproducibility and scalability assessment of fed-batch $\mathrm{CHO}$ cultures, Biotechnology and Bioengineering 106 (1) (2010) 57-67.

[268] R. Legmann, H. B. Schreyer, R. G. Combs, E. L. McCormick, A. P. Russo, S. T. Rodgers, A predictive high-throughput scale-down model of monoclonal antibody production in CHO cells, Biotechnology and Bioengineering 104 (6) (2009) 1107-1120.

[269] M. Sokolov, J. Ritscher, N. MacKinnon, J.-M. Bielser, D. Brühlmann, D. Rothenhäusler, G. Thanei, M. Soos, M. Stettler, J. Souquet, H. Broly, M. Morbidelli, A. Butté, Robust factor selection in early cell culture process development for the production of a biosimilar monoclonal antibody, Biotechnology Progress (2016) 1-11.

[270] R. McGill, J. W. Tukey, W. A. Larsen, Variations of Box Plots, The American Statistician Vol. 32 (1) (1978) 12-16.

[271] I. Jolliffe, Principal Component Analysis, Springer Series in Statistics, Springer-Verlag, New York, 2002.

[272] Y. Hou, C. Jiang, A. A. Shukla, S. M. Cramer, Improved process analytical technology for protein a chromatography using predictive principal component analysis tools, Biotechnology and Bioengineering 108 (1) (2011) 59-68.

[273] Y. E. Thomassen, E. N. M. Van Sprang, L. A. Van Der Pol, W. A. M. Bakker, Multivariate data analysis on historical IPV production data for better process understanding and future improvements, Biotechnology and Bioengineering 107 (1) (2010) 96-104.

[274] R. De Maesschalck, D. Jouan-Rimbaud, D. Massart, The Mahalanobis distance, Chemometrics and Intelligent Laboratory Systems 50 (1) (2000) 1-18.

[275] W. V. Moore, P. Leppert, Role of Aggregated Human Growth Hormone (hGH) in Development of Antibodies to hGH, The Journal of Clinical Endocrinology \& Metabolism 51 (4) (1980) 691-697.

[276] M. Sokolov, M. Soos, B. Neunstoecklin, M. Morbidelli, A. Butté, R. Leardi, T. Solacroup, M. Stettler, H. Broly, Fingerprint detection and process prediction by multivariate analysis of fed-batch monoclonal antibody cell culture data, Biotechnology Progress 31 (6) (2015) 1633-1644.

[277] H. Aghamohseni, Effect of Culture Conditions on the Glycosylation Patterns of mAb, Ph.D. thesis, University of Waterloo (2015). 
[278] S. N. Sou, C. Sellick, K. Lee, A. Mason, S. Kyriakopoulos, K. M. Polizzi, C. Kontoravdi, How does mild hypothermia affect monoclonal antibody glycosylation?, Biotechnology and Bioengineering 112 (6) (2015) 1165-1176.

[279] S. F. Abu-Absi, L. Yang, P. Thompson, C. Jiang, S. Kandula, B. Schilling, A. A. Shukla, Defining process design space for monoclonal antibody cell culture, Biotechnology and Bioengineering 106 (6) (2010) 894-905.

[280] Y. Fan, I. Jimenez Del Val, C. Müller, A. M. Lund, J. W. Sen, S. K. Rasmussen, C. Kontoravdi, D. Baycin-Hizal, M. J. Betenbaugh, D. Weilguny, M. R. Andersen, A multi-pronged investigation into the effect of glucose starvation and culture duration on fed-batch $\mathrm{CHO}$ cell culture, Biotechnology and Bioengineering 112 (10) (2015) 2172-2184.

[281] I. Jimenez del Val, Y. Fan, D. Weilguny, Dynamics of immature mAb glycoform secretion during CHO cell culture: An integrated modelling framework, Biotechnology Journal (2016) 610-623.

[282] I. J. del Val, K. M. Polizzi, C. Kontoravdi, A theoretical estimate for nucleotide sugar demand towards Chinese Hamster Ovary cellular glycosylation, Scientific Reports 6 (January) (2016) 28547.

[283] S. Kishishita, T. Nishikawa, Y. Shinoda, H. Nagashima, H. Okamoto, S. Takuma, H. Aoyagi, Effect of temperature shift on levels of acidic charge variants in IgG monoclonal antibodies in Chinese hamster ovary cell culture, Journal of Bioscience and Bioengineering 119 (6) (2015) 700-705.

[284] S. Dengl, M. Wehmer, F. Hesse, F. Lipsmeier, O. Popp, K. Lang, Aggregation and Chemical Modification of Monoclonal Antibodies under Upstream Processing Conditions, Pharmaceutical Research 30 (5) (2013) 1380-1399.

[285] S. Kang, Z. Zhang, J. Richardson, B. Shah, S. Gupta, C.-J. Huang, J. Qiu, N. Le, H. Lin, P. V. Bondarenko, Metabolic markers associated with high mannose glycan levels of therapeutic recombinant monoclonal antibodies, Journal of Biotechnology 203 (2015) $22-31$.

[286] L. Liu, Y.-X. Xu, K. L. Caradonna, E. K. Kruzel, B. A. Burleigh, J. D. Bangs, C. B. Hirschberg, Inhibition of Nucleotide Sugar Transport in Trypanosoma brucei Alters Surface Glycosylation, Journal of Biological Chemistry 288 (15) (2013) 10599-10615.

[287] A. Porat, Z. Elazar, Regulation of Intra-Golgi Membrane Transport by Calcium, Journal of Biological Chemistry 275 (38) (2000) 29233-29237.

[288] J. T. Powell, K. Brew, Metal ion activation of galactosyltransferase, The Journal of Biological Chemistry 251 (12) (1976) 3645-3652.

[289] A. Mironov, A. Luini, A. Mironov, A synthetic model of intra-Golgi traffic, FASEB Journal 12 (2) (1998) 249-252. 
[290] P. Hossler, L.-T. Goh, M. M. Lee, W.-S. Hu, GlycoVis: visualizing glycan distribution in the protein N-glycosylation pathway in mammalian cells, Biotechnology and Bioengineering 95 (5) (2006) 946-960.

[291] I. Hang, C. W. Lin, O. C. Grant, S. Fleurkens, T. K. Villiger, M. Soos, M. Morbidelli, R. J. Woods, R. Gauss, M. Aebi, Analysis of site-specific N-glycan remodeling in the endoplasmic reticulum and the Golgi, Glycobiology 25 (12) (2015) 1335-1349.

[292] T. K. Villiger, R. F. Steinhoff, M. Ivarsson, T. Solacroup, M. Stettler, H. Broly, J. Krismer, M. Pabst, R. Zenobi, M. Morbidelli, M. Soos, High-throughput profiling of nucleotides and nucleotide sugars to evaluate their impact on antibody N-glycosylation, Journal of Biotechnology 229 (2016) 3-12.

[293] T. K. Villiger, A. Roulet, A. Périlleux, M. Stettler, H. Broly, M. Morbidelli, M. Soos, Controlling the time evolution of $\mathrm{mAb} N$-linked glycosylation, Part I: Microbioreactor experiments, Biotechnology Progress 32 (5) (2016) 1123-1134.

[294] T. K. Villiger, E. Scibona, M. Stettler, H. Broly, M. Morbidelli, M. Soos, Controlling the time evolution of mAb N-linked glycosylation - Part II: Model-based predictions, Biotechnology Progress 32 (5) (2016) 1135-1148.

[295] I. Jimenez del Val, J. M. Nagy, C. Kontoravdi, A dynamic mathematical model for monoclonal antibody N-linked glycosylation and nucleotide sugar donor transport within a maturing Golgi apparatus, Biotechnology Progress 27 (6) (2011) 1730-1743.

[296] P. N. Spahn, A. H. Hansen, H. G. Hansen, J. Arnsdorf, H. F. Kildegaard, N. E. Lewis, A Markov chain model for $\mathrm{N}$-linked protein glycosylation - towards a low-parameter tool for model-driven glycoengineering, Metabolic Engineering 33 (2016) 52-66.

[297] J. Lisec, N. Schauer, J. Kopka, L. Willmitzer, A. R. Fernie, Gas chromatography mass spectrometry-based metabolite profiling in plants, Nature Protocols 1 (1) (2006) 387396.

[298] L. Eriksson, E. Johansson, N. Kettaneh-Wold, J. Trygg, C. Wikström, S. Wold, Multi- and Megavariate Data Analysis - Part I: Basic Principles and Applications, Umetrics, 2006.

[299] J. Selhub, Homocysteine Metabolism, Annual Review of Nutrition 19 (1) (1999) 217-246.

[300] C. R. Geren, L. M. Geren, K. E. Ebner, Inhibition and inactivation of bovine mammary and liver UDP galactose 4 epimerases, Journal of Biological Chemistry 252 (6) (1977) 2089-2094.

[301] P. Chen, S. W. Harcum, Effects of amino acid additions on ammonium stressed CHO cells, Journal of Biotechnology 117 (3) (2005) 277-286.

[302] J. M. I. Daenzer, R. D. Sanders, D. Hang, J. L. Fridovich-Keil, UDP-Galactose 4-Epimerase Activities toward UDP-Gal and UDP-GalNAc Play Different Roles in the Development of Drosophila melanogaster, PLoS Genetics 8 (5) (2012) e1002721. 
[303] H. Mehdizadeh, D. Lauri, K. M. Karry, M. Moshgbar, R. Procopio-Melino, D. Drapeau, Generic Raman-based calibration models enabling real-time monitoring of cell culture bioreactors, Biotechnology Progress 31 (4) (2015) 1004-1013.

[304] A. Datta-Mannan, L. Huang, J. Pereira, B. Yaden, A. Korytko, J. E. Croy, Insights into the impact of heterogeneous glycosylation on the pharmacokinetic behavior of follistatinFc-based biotherapeutics, Drug Metabolism and Disposition 43 (12) (2015) 1882-1890.

[305] Y. Kaneko, F. Nimmerjahn, J. V. Ravetch, Anti-inflammatory activity of immunoglobulin G resulting from Fc sialylation, Science 313 (5787) (2006) 670-673.

[306] G. Cartron, L. Dacheux, G. Salles, P. Solal-Celigny, P. Bardos, P. Colombat, H. Watier, Therapeutic activity of humanized anti-CD20 monoclonal antibody and polymorphism in IgG Fc receptor FcgammaRIIIa gene, Blood 99 (3) (2002) 754-758.

[307] M. Peipp, J. J. L. Van Bueren, T. Schneider-Merck, W. W. K. Bleeker, M. Dechant, T. Beyer, R. Repp, P. H. C. Van Berkel, T. Vink, J. G. J. Van De Winkel, P. W. H. I. Parren, T. Valerius, Antibody fucosylation differentially impacts cytotoxicity mediated by NK and PMN effector cells, Blood 112 (6) (2008) 2390-2399.

[308] S. Iida, R. Kuni-Kamochi, K. Mori, H. Misaka, M. Inoue, A. Okazaki, K. Shitara, M. Satoh, Two mechanisms of the enhanced antibody-dependent cellular cytotoxicity (ADCC) efficacy of non-fucosylated therapeutic antibodies in human blood, BMC Cancer 9 (9) (2009) 58.

[309] P. H. van Berkel, J. Gerritsen, E. van Voskuilen, G. Perdok, T. Vink, J. G. van de Winkel, P. W. Parren, Rapid production of recombinant human IgG with improved ADCC effector function in a transient expression system, Biotechnology and Bioengineering 105 (2) (2010) 350-357.

[310] M. Yu, D. Brown, C. Reed, S. Chung, J. Lutman, E. Stefanich, A. Wong, J. P. Stephan, R. Bayer, Production, characterization and pharmacokinetic properties of antibodies with N-linked Mannose-5 glycans, mAbs 4 (August) (2012) 475-487.

[311] W. Shatz, S. Chung, B. Li, B. Marshall, M. Tejada, W. Phung, W. Sandoval, R. F. Kelley, J. M. Scheer, Knobs-into-holes antibody production in mammalian cell lines reveals that asymmetric afucosylation is sufficient for full antibody-dependent cellular cytotoxicity, mAbs 5 (6) (2013) 872-881.

[312] K. Mori, S. Iida, N. Yamane-Ohnuki, Y. Kanda, R. Kuni-Kamochi, R. Nakano, H. ImaiNishiya, A. Okazaki, T. Shinkawa, A. Natsume, R. Niwa, K. Shitara, M. Satoh, Nonfucosylated therapeutic antibodies: the next generation of therapeutic antibodies, Cytotechnology 55 (2-3) (2007) 109-114.

[313] C. Ferrara, P. Brünker, T. Suter, S. Moser, U. Püntener, P. Umaña, Modulation of therapeutic antibody effector functions by glycosylation engineering: influence of 
Golgi enzyme localization domain and co-expression of heterologous beta1, 4-Nacetylglucosaminyltransferase III and Golgi alpha-mannosidase II, Biotechnology and Bioengineering 93 (5) (2006) 851-861.

[314] H. S. Lee, Y. Qi, W. Im, Effects of N-glycosylation on protein conformation and dynamics: Protein Data Bank analysis and molecular dynamics simulation study, Scientific Reports 5 (2015) 8926. 



\title{
Nomenclature
}

\author{
Abbreviations \\ 2AB-UPLC \\ 2-amino-benzamide ultra-performance liquid chromatography \\ 2F-p-fuc \\ 2F-peracetyl fucose or (3S,4R,5R,6S)-3-fluoro-6-methyltetrahydro-2H- \\ pyran-2,4,5-triyl triacetate \\ 4PL \\ Four parameter logistic \\ AAAS \\ Alkaline amino acid solution \\ $\mathrm{ACN}$ \\ Acetonitrile \\ ADCC \\ Antibody-dependent cell-mediated cytotoxicity \\ ADP \\ Adenosine diphosphate \\ AGE \\ Advanced glycation end products \\ Asn \\ Asparagine \\ ATP \\ Adenosine triphosphate \\ BIA \\ Biomolecular interaction analysis \\ BSA \\ Bovine serum albumin \\ $\mathrm{Clq}$ \\ Subunit of C1-complex \\ CDC \\ Complement-dependent cytotoxicity \\ $\mathrm{CDF}$ \\ Chemically-defined feed \\ CDP \\ CGE-LIF \\ Cytidine diphosphate \\ $\mathrm{CHO}$ \\ Capillary gel electrophoresis with laser-induced detection \\ Chinese Hamster Ovary \\ Ctrl \\ Control \\ Cys \\ Cysteine \\ DoE \\ Design of experiment \\ DT \\ Decision tree \\ DTT \\ Dithiothreitol \\ DWP \\ Deepwell plate \\ $\mathrm{EC}_{50}$ \\ Half-maximal effector concentration \\ ELISA \\ Enzyme-linked immunosorbent assay \\ ER \\ Endoplasmic reticulum
}


Fab Fragment antigen-binding, region on antibody binding to antigens

Fc Fragment crystallizable, region interacting with cell surface receptors

$\mathrm{Fc} \gamma \mathrm{R} \quad \mathrm{F} c \gamma$ receptor

Fuc Fucose

G6PD Glucose-6-phosphate dehydrogenase

Gal Galactose

GalNAc N-Acetylgalactosamine

GalT Galactosyltransferase

GDP Guanosine diphosphate

Glc Glucose

GlcNAc N-Acetylglucosamine

Gln Glutamine

Hex Hexose

HM High mannose

HMW High-molecular-weight species

HRP Horseradish peroxidase

IAA

Indole-3-acetic acid

IAM

2-iodoacetamide

IgG

Immunoglobulin G

Kif

Kifunensine

LacNAc

$\mathrm{N}$-acetyllactosamine

LMW

Low-molecular-weight species

$\mathrm{mAb}$

Monoclonal antibody

Man

Mannose

MES

2-(N-morpholino)ethanesulfonic acid

$\mathrm{Mn}$

Manganese

MS

MSE

Mass spectrometry

Mass spectrometry dynamically switching between low-energy collision-induced dissociation and high-energy collision-induced dissociation

MSX

Methionine sulfoximine

MVA

Multivariate analysis

NAC

$\mathrm{N}$-acetyl-cysteine

NFAT

Nuclear factor of activated T-cells

NGNA

$\mathrm{N}$-glycolylneuraminic acid

$\mathrm{NH}_{4}$

Ammonium

NS

Nucleotide sugar

PC

Principal component 


$\begin{array}{ll}\text { PCA } & \text { Principal component analysis } \\ \text { PK } & \text { Pharmacokinetics } \\ \text { PLS } & \text { Partial least square } \\ \text { QTOF } & \text { Quadrupole time of flight } \\ \text { rcf } & \text { Relative centrifugal force } \\ \text { RMP } & \text { Reference medicinal product } \\ \text { RMSECV } & \text { Root mean square error of cross validation } \\ \text { RMSEE } & \text { Root mean square error of evaluation } \\ \text { RMSEP } & \text { Root mean square error of prediction } \\ \text { RT } & \text { Room temperature } \\ \text { SAc } & \text { Acetylated sialic acid } \\ \text { Sia } & \text { Sialic acid } \\ \text { SPR } & \text { Surface plasmon resonance } \\ \text { ST } & \text { Shake tube } \\ \text { TE } & \text { Trace elements } \\ \text { TrxR } & \text { Thioredoxin reductase } \\ \text { UDP } & \text { Uridine diphosphate } \\ \text { Urd } & \text { Uridine } \\ \text { VC } & \text { Viable cells } \\ \text { VIP } & \text { Variable importance plot } \\ & \end{array}$

\section{Glycan Groups}

$\mathrm{AF}$

Afucosylated glycans

Fuc

Fucosylated glycans

Gal

Galactosylated glycans

HM

High mannose species

Misc

Miscellaneous glycans

Sial

Sialylated glycans

\section{Glycans}

A0

Al

A2

A2G1

A2G2

A2G2S1

A2G2S2
Man3GlcNAc2

GlcNAcMan3GlcNAc2

G0; GlcNAc2Man3GlcNAc2

GalGlcNAc2Man3GlcNAc2

Gal2GlcNAc2Man3GlcNAc2

SiaGal2GlcNAc2Man3GlcNAc2

Sia2Gal2GlcNAc2Man3GlcNAc2 


\begin{tabular}{|c|c|}
\hline A3 & GlcNAc3Man3GlcNAc2 \\
\hline A3G1S1 & SiaGalGlcNAc3Man3GlcNAc2 \\
\hline A3G2S1 & SiaGal2GlcNAc3Man3GlcNAc2 \\
\hline A3G2S2 & Sia2Gal2GlcNAc3Man3GlcNAc2 \\
\hline A3G3S1 & SiaGal3GlcNAc3Man3GlcNAc2 \\
\hline A3G3S2 & Sia2Gal3GlcNAc3Man3GlcNAc2 \\
\hline A3G3S3 & Sia3Gal3GlcNAc3Man3GlcNAc2 \\
\hline A3G3S3_1Ac & SAcSia2Gal3GlcNAc3Man3GlcNAc2 \\
\hline A4G3S2 & Sia2Gal3GlcNAc4Man3GlcNAc2 \\
\hline A4G3S3 & Sia3Gal3GlcNAc4Man3GlcNAc2 \\
\hline A4G4S2 & Sia2Gal4GlcNAc4Man3GlcNAc2 \\
\hline A4G4S3 & Sia3Gal4GlcNAc4Man3GlcNAc2 \\
\hline A4G4S4 & Sia4Gal4GlcNAc4Man3GlcNAc2 \\
\hline A4G4S4_1Ac & SAcSia3Gal4GlcNAc4Man3GlcNAc2 \\
\hline FAl & GlcNAcMan3GlcNAc2Fuc \\
\hline FA1G1 & GalGlcNAcMan3GlcNAc2Fuc \\
\hline FA1G1S1 & SiaGalGlcNAcMan3GlcNAc2Fuc \\
\hline FA2 & G0F; GlcNAc2Man3GlcNAc2Fuc \\
\hline FA2BG1 & GalGlcNAc3Man3GlcNAc2Fuc \\
\hline FA2G1 & G1F; GalGlcNAc2Man3GlcNAc2Fuc \\
\hline FA2G1S1 & SiaGalGlcNAc2Man3GlcNAc2Fuc \\
\hline FA2G2 & G2F; Gal2GlcNAc2Man3GlcNAc2Fuc \\
\hline FA2G2aG1S1 & SiaGal3GlcNAc2Man3GlcNAc2Fuc \\
\hline FA2G2S1 & SiaGal2GlcNAc2Man3GlcNAc2Fuc \\
\hline FA2G2S1(NGNA) & NGNAGal2GlcNAc2Man3GlcNAc2Fuc \\
\hline FA2G2S1_1Ac & SAcGal2GlcNAc2Man3GlcNAc2Fuc \\
\hline FA2G2S1_2Ac & $\begin{array}{l}\text { SAcGal2GlcNAc2Man3GlcNAc2Fuc (the } 2 \text { acetylations are on the same } \\
\text { sialic acid) }\end{array}$ \\
\hline FA2G2S2 & Sia2Gal2GlcNAc2Man3GlcNAc2Fuc \\
\hline FA2G2S2_1Ac & SAcSiaGal2GlcNAc2Man3GlcNAc2Fuc \\
\hline FA2G2S2_1NGNA & NGNASiaGal2GlcNAc2Man3GlcNAc2Fuc \\
\hline FA2G2S[6]1NGNA & NGNAGal2GlcNAc2Man3GlcNAc2Fuc \\
\hline FA3 & GlcNAc3Man3GlcNAc2Fuc \\
\hline FA3G1 & GalGlcNAc3Man3GlcNAc2Fuc \\
\hline FA3G2 & Gal2GlcNAc3Man3GlcNAc2Fuc \\
\hline FA3G2S1 & SiaGal2GlcNAc3Man3GlcNAc2Fuc \\
\hline FA3G2S2 & Sia2Gal2GlcNAc3Man3GlcNAc2Fuc \\
\hline FA3G3 & Gal3GlcNAc3Man3GlcNAc2Fuc \\
\hline
\end{tabular}




\begin{tabular}{|c|c|}
\hline FA3G3S1 & SiaGal3GlcNAc3Man3GlcNAc2Fuc \\
\hline FA3G3S2 & Sia2Gal3GlcNAc3Man3GlcNAc2Fuc \\
\hline FA3G3S2_1Ac & SAcSiaGal3GlcNAc3Man3GlcNAc2Fuc \\
\hline FA3G3S3 & Sia3Gal3GlcNAc3Man3GlcNAc2Fuc \\
\hline FA3G3S3_1Ac & SAcSia2Gal3GlcNAc3Man3GlcNAc2Fuc \\
\hline FA3G3S3_1NGNA & NGNASia2Gal3GlcNAc3Man3GlcNAc2Fuc \\
\hline FA3G3S3_2Ac & SAc2SiaGal3GlcNAc3Man3GlcNAc2Fuc \\
\hline FA4 & GlcNAc4Man3GlcNAc2Fuc \\
\hline FA4G1 & GalGlcNAc4Man3GlcNAc2Fuc \\
\hline FA4G1S1 & SiaGalGlcNAc4Man3GlcNAc2Fuc \\
\hline FA4G3S1 & SiaGal3GlcNAc4Man3GlcNAc2Fuc \\
\hline FA4G3S2 & Sia2Gal3GlcNAc4Man3GlcNAc2Fuc \\
\hline FA4G3S3 & Sia3Gal3GlcNAc4Man3GlcNAc2Fuc \\
\hline FA4G4 & Gal4GlcNAc4Man3GlcNAc2Fuc \\
\hline FA4G4L1 & GalGlcNAcGal4GlcNAc4Man3GlcNAc2Fuc \\
\hline FA4G4L1S2 & GalGlcNAcSia2Gal4GlcNAc4Man3GlcNAc2Fuc \\
\hline FA4G4L1S3 & GalGlcNAcSia3Gal4GlcNAc4Man3GlcNAc2Fuc \\
\hline FA4G4L1S4 & GalGlcNAcSia4Gal4GlcNAc4Man3GlcNAc2Fuc \\
\hline FA4G4S1 & SiaGal4GlcNAc4Man3GlcNAc2Fuc \\
\hline FA4G4S2 & Sia2Gal4GlcNAc4Man3GlcNAc2Fuc \\
\hline FA4G4S2_1Ac & SAcSiaGal4GlcNAc4Man3GlcNAc2Fuc \\
\hline FA4G4S3 & Sia3Gal4GlcNAc4Man3GlcNAc2Fuc \\
\hline FA4G4S3_1Ac & SAcSia2Gal4GlcNAc4Man3GlcNAc2Fuc \\
\hline FA4G4S3_1NGNA & NGNASia2Gal4GlcNAc4Man3GlcNAc2Fuc \\
\hline FA4G4S4 & Sia4Gal4GlcNAc4Man3GlcNAc2Fuc \\
\hline FA4G4S4_1Ac & SAcSia3Gal4GlcNAc4Man3GlcNAc2Fuc \\
\hline FA4G4S4_1NGNA & NGNASia3Gal4GlcNAc4Man3GlcNAc2Fuc \\
\hline FA4G4S4_2Ac & SAc2Sia2Gal4GlcNAc4Man3GlcNAc2Fuc \\
\hline FM3 & Man3GlcNAc2Fuc \\
\hline FM4A1G1 & GalGlcNAcMan4GlcNAc2Fuc \\
\hline FM5A1G1S1 & SiaGalGlcNAc1Man5GlcNAc2Fuc \\
\hline Hybrid-F & GalGlcNAcMan4GlcNAc2Fuc \\
\hline M4 or Man4 & Man4GlcNAc2 \\
\hline M5 or Man5 & Man5GlcNAc2 \\
\hline M6 or Man6 & Man6GlcNAc2 \\
\hline M7 or Man7 & Man7GlcNAc2 \\
\hline M8 or Man8 & Man8GlcNAc2 \\
\hline M9 or Man9 & Man9GlcNAc2 \\
\hline
\end{tabular}





\section{Scientific Contributions}

\section{Publications}

D. Brühlmann, A. Muhr, R. Parker, T. Vuillemin, B. Bucsella, S. Torre, F. La Neve, A. Lembo, T. Haas, M. Sauer, J. Souquet, H. Broly, J. Hemberger, M. Jordan, Cell Culture Media Supplemented with Raffinose Reproducibly Enhances High Mannose Glycan Formation, submitted.

D. Brühlmann, M. Sokolov, A. Butté, M. Sauer, J. Hemberger, J. Souquet, H. Broly, M. Jordan, Parallel Experimental Design and Multivariate Analysis Provides Efficient Screening of Cell Culture Media Supplements to Improve Biosimilar Product Quality, submitted.

M. Sokolov, J. Ritscher, N. MacKinnon, J.-M. Bielser, D. Brühlmann, D. Rothenhäusler, G. Thanei, M. Soos, M. Stettler, J. Souquet, H. Broly, M. Morbidelli, A. Butté, Robust factor selection in early cell culture process development for the production of a biosimilar monoclonal antibody, Biotechnology Progress (2016) [Epub ahead of print].

Y. Rouiller, J.-M. Bielser, D. Brühlmann, M. Jordan, H. Broly, M. Stettler, Screening and Assessment of Performance and Molecule Quality Attributes of Industrial Cell Lines Across Different Fed-batch Systems, Biotechnology Progress 32 (1) (2016) 160-170.

D. Brühlmann, A. Muhr, J. Hemberger, M. Sauer, H. Kornmann, M. Jordan, and H. Broly, The Potential of Small Molecules to Modulate Glycosylation by Media Design, BMC Proceedings 9 (2015) (Suppl9):P38

D. Brühlmann, M. Jordan, J. Hemberger, M. Sauer, M. Stettler and H. Broly, Tailoring Recombinant Protein Quality by Rational Media Design, Biotechnology Progress 31 (3) (2015) $615-629$.

\section{Oral Presentations}

D. Brühlmann, J. Souquet, M. Sauer, H. Broly, J. Hemberger, M. Jordan, Tailoring N-Glycosylation by Rational Cell Culture Medium Design, GlycoBioTec 2017, Berlin, Germany, February 2017. 
M. Stettler and D. Brühlmann, Modulating Glycosylation by Media Design, JAACT 2016, Kobe, Japan, November 2016.

D. Brühlmann, M. Jordan, A. Muhr, R. Parker, J. Hemberger, M. Sauer, J. Souquet, H. Broly, Modulating Glycosylation by Media Design, Cell Culture \& Bioprocessing Summit, London, UK, November 2016.

D. Brühlmann, M. Jordan, G. Leclerq, A. Muhr, J. Hemberger, M. Sauer, J. Souquet, H. Broly, The Potential of Small Molecules to Modulate Glycosylation by Media Design, Cell Culture Engineering XV, Palm Springs CA, USA, May 2016.

D. Brühlmann, M. Jordan, J. Hemberger, M. Sauer, M. Stettler, H. Broly, Modulating Glycosylation by Media Design, Bioprocessing International, Vienna, Austria, April 2016.

D. Brühlmann, M. Jordan, A. Muhr, R. Parker, J. Hemberger, M. Sauer, M. Stettler, H. Broly, Modulating Glycosylation by Media Design, Cell Culture World, Munich, Germany, February 2016.

D. Brühlmann, M. Jordan, A. Muhr, R. Parker, J. Hemberger, M. Sauer, M. Stettler, H. Broly, The Potential of Small Molecules to Modulate Glycosylation by Media Design, 2015 AIChE Annual Meeting, Salt Lake City UT, USA, November 2015.

D. Brühlmann, J.-M. Bielser, M. Jordan, Y. Rouiller, M. Stettler, H. Broly, Assessment of Performance and Molecule Quality Attributes of Cell Lines in Fed-batch, Bioprocessing Summit, Boston MA, USA, August 2015.

D. Brühlmann, M. Jordan, Efficient Cell Culture Media Optimization, Bioprocessing Summit, Boston MA, USA, August 2015.

D. Brühlmann, M. Jordan, J. Hemberger, M. Sauer, H. Kornmann and H. Broly, The Cell—An Amazing Artist: Media Supplementation Is an Attractive Way to Tune Glycosylation, MerckSerono Bioprocess Workshop, Montreux, Switzerland, May 2015.

\section{Posters}

B. Bucsella, A. Fornage, C. Le Denmat, D. Brühlmann and F. Kálmán, Nucleotide and Nucleotide Sugar Quantification in CHO Cell Extracts by Capillary Electrophoresis, Swiss Chemical Society (SCS) Fall Meeting 2016, Zurich, Switzerland, September 2016.

D. Brühlmann, A. Muhr, J. Hemberger, M. Sauer, H. Kornmann, M. Jordan and H. Broly, The Potential of Small Molecules to Modulate Glycosylation by Media Design, $24^{\text {th }}$ ESACT Meeting, Barcelona, Spain, May/June 2015.

N. Stankiewicz, D. Brühlmann, J. Bleifuss, A. Simon, S. Schüssler, T. Wicht, T. Scaramuzza and J. von Hagen, Compactation of Cell Culture Media-A New Technology Application to 
Leverage the Advantages of Dry Powder Formulations, $24^{\text {th }}$ ESACT Meeting, Barcelona, Spain, May/June 2015. 



\section{Declaration of Authorship}

I hereby declare that my thesis entitled "Tailoring Recombinant Protein Quality by Rational Media Design" is the result of my own work and that I have used no other sources nor resources except where stated otherwise. Chapter 7 is the result of a collaboration with the Institute of Chemical and Bioengineering, Department of Chemistry and Applied Biosciences of the Swiss Federal Institute of Technology (ETH Zurich). I played a major role in the preparation and execution of the experiment. The multivariate data analysis was performed by ETH Zurich as referenced in the Materials and Methods part.

I did not receive any help or support from commercial consultants. I confirm that the work has neither been submitted in identical nor in similar form to any other examination procedure.

Würzburg, February 16, 2017

David Brühlmann 